Motivation and Emotional Distraction

MOTIVATION REDUCES POSITIVE AND NEGATIVE EMOTIONAL DISTRACTIONS

BY

AMY T. WALSH

A thesis submitted to the Victoria University of Wellington

in fulfilment of the requirements for the degree of

Doctor of Philosophy in Cognitive and Behavioural Neuroscience.

Victoria University of Wellington 2019 
Motivation and Emotional Distraction

\begin{abstract}
Attention is biased toward emotional stimuli, which are often important for our biologically-determined goals of survival and reproduction. But to succeed in our daily tasks we must sometimes ignore emotional stimuli that are not relevant to current goals. In four experiments, I examine the extent to which we can ignore emotional stimuli if we are motivated to do so. I draw on the Dual Mechanisms of Control (DMC) framework which proposes that we use two modes of control to deal with distraction: reactive control, which shifts attention back to a task after distraction has occurred; and proactive control, which allows us to anticipate and control distraction before it occurs. In non-emotional contexts, task motivation encourages use of more effective, but more effortful, proactive control to ignore emotionally-neutral distractions. But, little is known about how we can control our attention to ignore highly distracting emotional stimuli. In all experiments, participants completed a simple visual task while attempting to ignore task-irrelevant negative (mutilation scenes), positive (erotic scenes), and neutral images (scenes of people). Distraction was indexed by slowing on distractor trials relative to a scrambled distractor, or no distractor, baseline. To manipulate motivation, half the participants completed the task with no performance-contingent reward; the other half completed the task with the opportunity to earn points and/or money for fast and accurate performance. In Experiment 1 the images were presented centrally, so attention must be shifted from the distractor location to complete the task. Reward reduced distraction by both positive and negative emotional images. Experiment 2 replicated Experiment 1, and measured pupil dilation to index the timecourse of cognitive effort. The aim of Experiment 2 was to determine whether motivation elicits a shift to proactive control to reduce emotional distraction, as it does in non-emotional contexts. Again, reward reduced positive and negative distraction. Pupil findings indicated that reward dynamically enhanced proactive control prior to stimulus-onset, facilitating rapid disengagement from distractors, regardless of their expected emotional value. In contrast, a sustained proactive strategy was used across blocks in which emotional distractors were expected, relative to blocks in which neutral distractors were expected. In the final two experiments, the distractors were presented peripherally and so must capture attention away from the central targets to impair performance. In Experiment 3, and in Experiment 4 - in which the points did not represent money - reward reduced attentional capture by positive and negative emotional distractors. Together, findings show that motivation can enhance control of positive and negative distractions that appear both centrally, and peripherally. Findings extend the DMC framework to an emotional context; motivation elicits a shift to proactive control, even when distractors are high arousal emotional stimuli. Further, in three out of four experiments, reward reduced emotional to a greater extent than neutral distraction, consistent with reward altering the outcome of goal-driven attentional competition between the targets and distractors. Understanding the complex interactions between motivation, emotion, and cognitive control will help to elucidate how we successfully navigate the world to achieve our goals.
\end{abstract}


Motivation and Emotional Distraction

\section{Acknowledgements}

Thanks to the many current, previous, and honourary members of the Cognitive Affective Neuroscience Lab, especially to everyone who piloted my experiments, gave feedback at lab meetings, and to those who helped in a multitude of other ways. It is a pleasure working with you all. Thanks especially to Hazel Godfrey, for being a wonderful friend and supportive lab mate. You've always challenged me to be a more thoughtful and conscientious researcher. Also a massive thank you to all the volunteers and students who helped me collect data for various projects: Lauren Bell, Nicky Duff, Hannah Quigan, Petra Bolitho, and Anne Rijnink. Thanks to Kealagh Robinson, Lauren Bell, Laura Kranz, Hazel Godfrey, and Hanna Squire, for each proofreading a chapter of my thesis while you were supposed to be on holiday.

Thank you to my two secondary supervisors, David Carmel and Dave Harper, for your valuable input along the way.

Thanks to the Marsden Fund NZ for funding my PhD.

Thanks to the staff at The Arborist, especially Hannah Daken, for providing me with a comfortable and friendly space to use as an office, and for the many coffees, chats, and wines. Thanks to the staff at Up Fitness, especially Hannah Challis, for giving me a daily escape, and keeping me sane and healthy.

The past four years have been up and down, with this past year being particularly difficult. Thanks to everyone who ensured I had a roof over my head this past year: Hannah Daken, Daniel Johnston, Anna Currie, Nick Anderson, Rosie Chittenden, Kieran Brown, Alana Oakly, Lynette Squire, and Russell Buchanan. Quite the group effort. I am so fortunate to have such an excellent group of friends. Thanks especially to: Hannah Daken, Alana Oakly, Robyn Zwaan, Rosie Woods, Anna Currie, Nick Anderson, Sarah Bradbury, Hanna Squire, Claire Krejcisz, James Beath, Tel McCubbin, Aviva Stein, Will Arlidge, Lauren Bell, and Chelsea Rose, for all the hangs, chats, dinners, distractions, and support. You're all beautiful people who make my life fun, and I love you all.

To my wonderful, loving family: Mum, Dad, David, Katie, and Elise. I could never thank you enough for your unconditional support and care in all its various forms, and for your unwavering confidence in me.

Gina, thank you for all that you have done for me over the years. You are an inspiring and creative teacher, and you have taught me how to be a rigorous, transparent, and curious researcher. Thank you for staying in touch when I went to the UK, and for the opportunity to do this $\mathrm{PhD}$. You prioritised my well-being over my work last year, while also ensuring I was doing what needed to be done. Your confidence in me gave me the strength to keep going. I am so lucky to have you as a mentor. 
Motivation and Emotional Distraction

\section{Research in this thesis appears in the following publications}

Experiments 1 and 2, from Chapters 2 and 3, of this thesis are published (details below). The manuscripts were adapted for this thesis in order to form a coherent whole. Any deviations from the published manuscript in the Results are noted in the Chapters. Experiments from Chapter 4 are currently in preparation to submit for publication. Although I am lead author of these three manuscripts, I will use the term "we" when discussing material related to these manuscripts throughout the thesis to acknowledge the contributions of my coauthors.

\section{Chapter 2}

Portions of this chapter appear in: Walsh, A. T., Carmel, D., Harper, D., \& Grimshaw, G. M. (2018). Motivation enhances control of positive and negative emotional distractions. Psychonomic Bulletin \& Review, 25(4), 1556-1562. https://doi.org/10.3758/s13423-017-1414-5

Prof. David Harper assisted with experimental design. Assoc. Prof. Gina Grimshaw and Dr. David Carmel assisted with experimental design, and provided feedback on the written manuscript. All authors approved the final version of the submitted manuscript.

Preprint of published manuscript can be accessed at https://osf.io/5kcvu/ and in Appendix A of this thesis.

\section{Chapter 3}

Portions of this chapter appear in: Walsh, A. T., Carmel, D., \& Grimshaw, G. M. (2018). Reward elicits cognitive control over emotional distraction: Evidence from pupillometry. Cognitive, Affective, \& Behavioural Neuroscience. https://doi.org/10.3758/s13415-018-00669-w

Assoc. Prof. Gina Grimshaw and Dr. David Carmel assisted with experimental design, and provided feedback on the written manuscript. All authors approved the final version of the submitted manuscript. Christel Devue and Al Abenoja helped with programming the pupil experiment.

Preprint of published manuscript: https://psyarxiv.com/k4z6u/, and Appendix C of this thesis. Link to preregistration: https://osf.io/jd96p/, and Appendix D of this thesis.

\section{Chapter 4}

The data from this chapter are not yet published. The manuscript in preparation lists as coauthors: Assoc. Prof. Gina Grimshaw, Dr. David Carmel, Prof. David Harper, and Petra Bolitho.

Assoc. Prof. Grimshaw assisted with experimental design and with feedback on writing. Dr. David Carmel assisted with experimental design. Prof. David Harper advised on the reward manipulation. Petra Bolitho assisted with data collection. 
Motivation and Emotional Distraction

Table of Contents

Page

Title Page

Abstract 2

Acknowledgements 3

Research in this thesis appears in the following publications 4

Table of Contents 5

List of Tables 7

List of Figures

\section{Chapter 1: General Introduction}

Thesis Overview

Attentional biases to emotional information 11

Cognitive control and the Dual Mechanisms of Control framework 21

The influence of reward on cognitive control 23

The influence of motivation on cognitive control 25

The influence of motivation on emotional distraction 29

The thesis experiments 32

Chapter 2. Motivation enhances control of positive and negative emotional distractions.

Experiment 1 Introduction 36

Experiment 1 Method 40

Experiment 1 Results 45

Experiment 1 Discussion 50

Chapter 3. Reward elicits cognitive control over emotional distraction:

Evidence from pupillometry.

Experiment 2 Introduction 54

Experiment 2 Method 58

Experiment 2 Results 64

Experiment 2 Discussion 81

Chapter 4. The influence of monetary and non-monetary reward on attentional capture by peripheral emotional distractors.

Chapter 4 Introduction 87

Experiment 3 Introduction: Does monetary reward attenuate attentional capture by peripheral emotional images? 
Motivation and Emotional Distraction

Experiment 3 Method 89

Experiment 3 Results and Discussion 92

Experiment 4 Introduction: Does non-monetary reward attenuate

attentional capture by peripheral emotional images? 96

Experiment 4 Method 100

Experiment 4 Results and Discussion 101

Chapter 4 General Discussion 107

Chapter 5: General Discussion 112

Does motivation enhance control of emotional and neutral distractions

to different degrees?

Does motivation enhance control of emotional distractions via a shift

to proactive control?

Does motivation enhance control of emotional distractors when they appear outside the current spatial locus of attention, as well as within the current spatial locus of attention?

Does motivation enhance control of positive and negative distractions

to different degrees? 122

Some thoughts on reward 124

Limitations and future directions 126

Applications 128

Conclusions 129

References 132

Appendices 159 
Motivation and Emotional Distraction

\section{List of Tables}

\section{Chapter 2}

Table 2.1. Mean IAPS valence and arousal ratings for each valence image set for men, and for women.

Table 2.2. Comparisons of mean correct RTs (ms) and accuracy (proportion correct) for scrambled versus intact images (distraction indices in $\mathrm{ms}$ ), for each distractor valence condition, separately for control and reward groups for Experiment 1.

\section{Chapter 3}

Table 3.1. Comparisons of mean correct RTs (ms) and accuracy (proportion correct) for scrambled versus intact images (distraction indices in $\mathrm{ms}$ ), for each distractor valence condition, separately for control and reward groups for Experiment 2.

Table 3.2. Mean pupil area in arbitrary units for preregistered tonic and phasic proactive time-windows, for each distractor valence condition, separately for control and reward groups.

Table 3.3. Comparisons of mean pupil area for the preregistered post-stimulus indices, for scrambled versus intact images, for each distractor valence condition, separately for control and reward groups.

\section{Chapter 4}

Table 4.1. Mean correct RTs (ms), accuracy (proportion correct), and distraction indices [distractor-present RT - distractor-absent RT] and [distractor-absent proportion correct distractor-present proportion correct] for each distractor valence condition, separately for control and reward groups, for Experiment 3.

Table 4.2. Mean correct RTs (ms), accuracy (proportion correct), and distraction indices [distractor-present RT - distractor-absent RT] and [distractor-absent proportion correct distractor-present proportion correct] for each distractor valence condition, separately for control and reward groups, for Experiment 4. 
Motivation and Emotional Distraction

\section{List of Figures}

\section{Chapter 2}

Figure 2.1. Trial sequence and target display for the central distractor task in Experiment 1 .

Figure 2.2. A still image of the animation of coins falling into piles, presented to reward participants between blocks.

Figure 2.3. Mean behavioural distraction indices (in $\mathrm{ms}$ ) by reward group and distractor valence for Experiment 1.

\section{Chapter 3}

Figure 3.1. Target display and trial sequence for the central distractor task in Experiment 2, and preregistered time-windows for pupil analyses.

Figure 3.2. Mean behavioural distraction indices (in $\mathrm{ms}$ ) by reward group and distractor valence for Experiment 2.

Figure 3.3. Mean pupil area (in arbitrary units) across the entire trial, separately for the reward and control groups.

Figure 3.4. Mean pupil area (in arbitrary units) across the $1000 \mathrm{~ms}$ tonic time-window, separately for the reward and control groups.

Figure 3.5. Mean pupil area (arbitrary units) across the $2000 \mathrm{~ms}$ pre-stimulus phasic timewindow, separately for the reward and control groups.

Figure 3.6. Mean pupil area (arbitrary units) across the $4100 \mathrm{~ms}$ post-stimulus phasic timewindow, separately for the reward and control groups.

Figure 3.7. Functional data analysis of mean pupil area (arbitrary units) across the $2000 \mathrm{~ms}$ pre-stimulus phasic time-window.

Figure 3.8. Functional data analysis of mean pupil area (arbitrary units) across the $4100 \mathrm{~ms}$ post-stimulus phasic time-window.

Figure 3.9. Correlations of pupil indices with behavioural distraction indices.

\section{Chapter 4}

Figure 4.1. Trial sequence for participants in the reward group, and the target display for the peripheral distraction task, for Experiments 3 and 4.

Figure 4.2. Mean behavioural distraction indices (in $\mathrm{ms}$ ) by reward group and distractor valence for Experiment 3.

Figure 4.3. Mean behavioural distraction indices (in $\mathrm{ms}$ ) by reward group and distractor valence for Experiment 4. 
Motivation and Emotional Distraction

\section{Chapter 1: General Introduction}

\section{Thesis Overview}

Emotion and motivation are the fundamental drivers of human behaviour (Darwin, 1869). They modulate our cognition - influencing perception, attention, memory and decision-making - to shape our behaviour in adaptive ways (Okon-Singer, Hendler, Pessoa, \& Shackman, 2015; Pessoa, 2008). Motivation provides us with the impetus to work towards our goals, leading us to rewards and away from harm; emotions often signal the presence of a potential opportunity or challenge that may require action. We therefore prioritise attention to emotional stimuli to serve our most fundamental, biologically-determined, goals of surviving and reproducing (Bradley, Codispoti, Cuthbert, \& Lang, 2001; Lang \& Bradley, 2010; LeDoux, 2012; Rolls, 2000). But at times, prioritising emotional information can be detrimental; when emotional stimuli are not relevant to achieving our current goals, and do not require our immediate action, they can nonetheless distract us from our task at hand (Carretié, 2014; Yiend, 2010). For example, if we go to the supermarket with the intention of only buying ingredients to make a healthy meal, our attention might be diverted by tempting but unhealthy items like chocolate, cigarettes, or wine. Or, while trying to work, we might be distracted by negative thoughts, social media notifications, or a colleague speaking loudly on the phone. Sometimes it seems we can shield ourselves from emotional distractions with relative ease, and at other times it can feel almost impossible. To what extent can motivation help us to succeed on our relatively mundane daily tasks in the face of exciting or aversive distractors?

To ignore distractions, and attend to currently goal-relevant information, we use a constellation of processes that are collectively referred to as cognitive control (Cohen, 2017; Gratton, Cooper, Fabiani, Carter, \& Karayanidis, 2018; Miller, 2000). The vast majority of research on cognitive control, and the factors that aid or impair it, have been conducted in non-emotional contexts. In these emotionally-neutral contexts, increasing task motivation by providing performance-contingent reward - enhances cognitive control, and thereby reduces distraction (Botvinick \& Braver, 2015; Yee \& Braver, 2018). The influential Dual Mechanisms of Control (DMC) framework (Braver, 2012; Braver, Gray, \& Burgess, 2007) posits that this upregulation of cognitive control often occurs via a shift in the timing of control mechanisms. Usually, when we encounter distractions, they capture our attention, after which a succession of control processes shift our attention back to whatever we were doing. This mode of operating is referred to as reactive control. Sometimes, when it is deemed worthwhile to do so (Kool, Shenhav, \& Botvinick, 2017; Shenhav, Botvinick, \& 
Motivation and Emotional Distraction

Cohen, 2013), we shift to a proactive control strategy; implementing control processes in anticipation of distraction. Proactive control requires more effort, but is a more effective shield against distraction.

One factor that can shift the balance in favour of using effortful proactive control is availability of rewards for successful task performance. It is well established that increasing task motivation elicits a shift to proactive control in emotionally-neutral contexts (Botvinick \& Braver, 2015; Yee \& Braver, 2018). But little is known about whether, and how, motivation influences cognitive control when distractors are emotional. We have strong attentional biases to emotional stimuli, even when they are not relevant to current task goals (e.g., Grimshaw, Kranz, Carmel, Moody, \& Devue, 2018; Okon-Singer, Tzelgov, \& Henik, 2007), because of their importance for surviving. Emotional stimuli might even bias attention via an emotion-specific mechanism (Pourtois, Schettino, \& Vuilleumier, 2013; Vuilleumier, 2015), in which case it follows that control of emotional and non-emotional distraction might occur via different mechanisms. But despite our bias to attention to emotion, sometimes we can ignore emotional distractions. For example, when attentional resources are consumed by a difficult task (Okon-singer et al., 2007; Pessoa, 2005), or when distractors occur frequently (Grimshaw et al., 2018). However, it remains unclear whether we can voluntarily control our attention to ignore emotional distractions - for example, if we are motivated to do so. The DMC framework may provide some clues to the puzzle of why we can sometimes ignore emotional content, and sometimes not - perhaps we switch to using more effective proactive control to ignore emotional distractors when it is deemed worthwhile to do so.

In this thesis, I examine whether the beneficial influence of motivation on cognitive control can be extended to contexts in which distractors are highly arousing positive and negative emotional stimuli, and probe the mechanisms of control in emotional contexts. All experiments in this thesis use the same basic paradigm. The task produces large emotional distraction effects under conditions of no reward. It is perceptually simple, so attentional resources are available to process the distractors. Moreover, distractors are presented relatively rarely ( $25 \%$ of trials), which is thought to elicit a reactive control strategy. Participants do a simple visual perceptual task while attempting to ignore emotional and neutral distractors that appear occasionally. In each experiment, half the participants complete the task with no performance-contingent reward, and the other half have the opportunity to earn rewards for fast and accurate performance. The key question is whether motivation enhances control of emotional distraction at all. A secondary question is whether motivation reduces emotional distraction by eliciting a shift to using proactive control. I also 
Motivation and Emotional Distraction

examine whether the benefit of motivation on distraction is dependent on distractor arousal (i.e., whether distractors are emotional or neutral); distractor valence (i.e., whether distractors are positive or negative); whether distractors are presented within or outside the spatial locus of attention (i.e., whether distractors are presented peripherally, or centrally); and the nature of the reward manipulation (monetary or non-monetary).

In the remainder of Chapter 1, I review the relevant literature on attentional biases to emotional information; cognitive control and the DMC framework; the influence of reward on cognitive control; the influence of motivation on cognitive control; and finally, the influence of motivation on control of emotional distractions. I then describe the four experiments included in this thesis, and outline the main hypotheses. In Chapter 2, I describe an experiment (Experiment 1) that examines whether motivation enhances control of positive and negative emotional distractions that appear within the current spatial locus of attention (that is, the central location where participants are directed to look). In Chapter 3 (Experiment 2), I replicate the previous experiment, and extend the question to whether motivation reduces emotional distraction via a shift in the timing of control mechanisms. Specifically, I use pupil dilation as an online index of the timecourse of cognitive control (van der Wel \& van Steenbergen, 2018), to examine whether motivation upregulates proactive control mechanisms in anticipation of distraction. In Chapter 4, I describe two experiments (Experiments 3 and 4) which examine whether motivation reduces attentional capture by emotional distractions that appear outside the current spatial locus of attention. Experiments 1, 2 and 3 manipulate motivation by providing points (to those in the reward group) for fast and accurate performance; these points represent greater monetary earnings on task completion. In contrast, in Experiment 4, the performance-contingent points are purely symbolic and do not represent monetary reward. In Chapter 5, I summarise and discuss the findings from all four experiments, to address the question of whether, and how, motivation enhances control of distraction in emotional contexts.

\section{Attentional biases to emotional information}

Of all the stimuli competing for our awareness (Desimone \& Duncan, 1995), those with emotional value are particularly effective at capturing our attention (Okon-Singer, Lichtenstein-Vidne, \& Cohen, 2013; Pourtois et al., 2013; Vuilleumier, 2015; Yiend, 2010). Emotional stimuli can be biologically-determined unconditioned reinforcers (e.g., food, sexual stimuli) or threats (e.g., spiders, snakes); but emotional stimuli can also gain their value by associative learning (e.g., money, guns; Brosch, Pourtois, \& Sander, 2010; LeDoux, 2012). Emotional stimuli elicit various subjective, physiological, and behavioural changes to 


\section{Motivation and Emotional Distraction}

prepare us for action, activating appetitive or defensive motivational circuits that guide us to approach rewards and retreat from potential threats (Bradley, 2009; Frijda, 1986; Lang \& Bradley, 2010; Rolls, 2000). Attentional biases to emotional information have been demonstrated by findings in a variety of cognitive tasks in which performance is enhanced when emotional stimuli are relevant to current goals (i.e., to completing the task). However, these biases have a cost to performance when the emotional stimuli are irrelevant to current goals (i.e., when the emotional content is not relevant to completing the task; Augst, Kleinsorge, \& Kunde, 2014; Burra, Pittet, Barras, \& Kerzel, 2018; Egner, Etkin, Gale, \& Hirsch, 2008; Erthal et al., 2005; Grimshaw et al., 2018; Gupta, Hur, \& Lavie, 2016; Most, Chun, Widders, \& Zald, 2005; Murphy, Devue, Corballis, \& Grimshaw, 2018; Okon-Singer et al., 2007; Padmala \& Pessoa, 2014; Padmala, Sambuco, Codispoti, \& Pessoa, 2018; Padmala, Sirbu, \& Pessoa, 2017; Parra, Sánchez, Valencia, \& Trujillo, 2018; Schmidt, Belopolsky, \& Theeuwes, 2015; Yates, Ashwin, \& Fox, 2010). The majority of findings demonstrate biases to attend to aversive or threatening negative emotional stimuli (e.g., taboo words, fearful or angry faces, images of snakes, weapons, or mutilations). Much of the research has sampled from anxious or depressed populations, who fall on the extreme end of the spectrum in terms of negative emotional biases (Bar-Haim, Lamy, Pergamin, BakermansKranenburg, \& van IJzendoorn, 2007; De Raedt \& Koster, 2010; Yiend, 2010). However, because these biases are a fundamental feature of our cognitive systems, they are also present in non-clinical populations. Below, I will review findings that index emotional biases in attention from a variety of cognitive tasks, focussing particularly on studies sampling from non-clinical populations.

A vast literature exists examining attentional biases to emotional stimuli in visual search tasks. In these tasks, participants respond as quickly as possible to a target stimulus amongst distractors. Emotional targets can be present within arrays of neutral distractors; or neutral targets can be presented within arrays of emotional distractors. Negative emotional stimuli are detected more efficiently than neutral ones when they are task-relevant targets of search, and interfere with performance when they are task-irrelevant distractors (Eastwood, Smilek, \& Merikle, 2001; Juth, Lundqvist, Karlsson, \& Öhman, 2005; Öhman, Flykt, \& Esteves, 2001). Both biologically-relevant threat stimuli (e.g., snakes), and those that gain negative valence acquired via learning (e.g., weapons), are detected more efficiently than neutral images (e.g., mushrooms; Fox, Griggs, \& Mouchlianitis, 2007).

In an emotional Stroop task, as in a traditional Stroop task (Stroop, 1935), participants respond as quickly as possible to the colour of words, while attempting to ignore 


\section{Motivation and Emotional Distraction}

the word's meaning. But in an emotional Stroop task, the stimuli are emotional and neutral words. Thus, the emotional content of the word is task-irrelevant, but the colour of the word is task-relevant. Slowing of responses when words are emotional, versus when they are neutral, measures the extent to which the emotional content interferes with performance. For example, task performance is impaired for taboo words, relative to neutral words (Siegrist, 1995). In non-clinical samples, emotional interference is usually only observed when words of one emotional valence are consistently presented within a block of trials (i.e., the words are blocked by valence; Bar-Haim et al., 2007), but not when words of varied emotional valence are intermixed within a block, suggesting a role of expectations in this emotional bias. In clinical samples (e.g., those with mood disorders), emotional biases are larger, and are specific in the sense that words that are relevant to an individual's concerns cause greater interference (e.g., spider-related words in spider phobics; Watts, McKenna, Sharrock, \& Trezise, 1986). Thus, the emotional Stroop effect is influenced by the motivational value of the word's meaning.

Cueing tasks (e.g., Armony \& Dolan, 2002; Pourtois, Grandjean, Sander, \& Vuilleumier, 2004) are used to examine the spatial allocation of attention to emotional and neutral information. In cueing paradigms, participants respond as quickly and accurately as possible to a target stimulus that appears in the same or different location as a cue (that is sometimes emotional). For example, in the dot probe task, an emotional and a neutral cue are presented simultaneously and briefly, side by side. If target detection is faster at the location of the emotional cue relative to the neutral cue, this is interpreted as an emotional bias. Attentional biases to threat stimuli are often observed in clinically-anxious individuals and those with high trait anxiety, especially when the stimuli match the specific concerns of the individual (Bar-Haim et al., 2007; Mathews \& MacLeod, 2005; Mogg, Bradley, \& Williams, 1995). However, emotional biases are less reliably observed in non-clinical populations (e.g., Mogg et al., 1995). At times, non-clinical samples even show avoidance of threatening stimuli (Bar-Haim et al., 2007).

All the tasks described above suffer from some problems, including small and/or unreliable biases in non-clinical samples (especially in the dot probe task; Chapman, Devue, \& Grimshaw, 2017); and importantly, it is not known which specific orienting mechanisms the tasks measure. Orienting refers to the processes by which attentional selection can occur: i.e., disengagement, shifting, and engagement of attention. Disengagement refers to withdrawal of attention from an attended stimulus; shifting refers to the process of relocating spatial attention; and engagement refers to facilitated processing of an attended stimulus 
Motivation and Emotional Distraction

(Posner \& Cohen, 1984; Posner, 1980, 2016). Researchers developed single cueing paradigms to more specifically study the orienting mechanisms underlying emotional biases of attention. In single cueing paradigms, a neutral or emotional cue appears, followed by a target that appears either in the same (on valid trials), or different (on invalid trials), location to the cue. On valid trials, the difference in response time on emotional versus neutral cue trials is thought to reflect attentional engagement to the emotional cue; on invalid trials the difference is thought to reflect slowed disengagement from the emotional cue. Using this paradigm, both facilitated engagement, and impaired disengagement, have been implicated in attentional biases to emotion (Koster, Verschuere, Burssens, Custers, \& Crombez, 2007; Vogt, De Houwer, Koster, Van Damme, \& Crombez, 2008).

The above paradigms examine how attention is allocated in space, but attention is also allocated over time. Rapid serial visual presentation paradigms examine how emotional information consumes attention over time. In these tasks, a rapid stream of stimuli are presented at one location. Some of the stimuli are task-relevant targets, and some are taskirrelevant distractors. If one target is presented close in time to another target, processing of the second target is often impaired (this is called an "attentional blink"; Broadbent \& Broadbent, 1987; Chun \& Potter, 1995; Raymond, Shapiro, \& Arnell, 1992). The blink is thought to be due to attentional resources being occupied by processing the first target. When the second target is emotional, it is more likely to break through to awareness (Anderson \& Phelps, 2001; Ihssen \& Keil, 2009; Mathewson, Arnell, \& Mansfield, 2008). In a related paradigm, an irrelevant emotional image is presented shortly before a neutral target image in a stream of images. Attentional resources are thought to be consumed by processing the emotional distractor, so people are more likely to miss the subsequent target. This effect is termed emotion-induced blindness (Most et al., 2005; Most, Smith, Cooter, Levy, \& Zald, 2007).

Although the vast majority of research has focussed on attentional biases to negative emotional stimuli, positive stimuli are also prioritised (Brosch, Pourtois, \& Sander, 2010; Brosch, Sander, \& Scherer, 2007; Pool, Brosch, Delplanque, \& Sander, 2016). The emotional value of a given stimulus sits on two orthogonal dimensions: valence (from negative to positive), and arousal (from low to high intensity; Gerber et al., 2008; Lang, Greenwald, Bradley, \& Hamm, 1993; Posner, Russell, \& Peterson, 2005). With some exceptions (e.g., anger; Harmon-Jones, 2003), we are motivated to withdraw from negative stimuli and to approach positive stimuli (Bradley et al., 2001; Lang \& Bradley, 2010; LeDoux, 2012; Rolls, 2000). Until recently, studies examining attentional biases to both positive and negative 


\section{Motivation and Emotional Distraction}

stimuli have often compared high arousal negative stimuli (e.g., images of mutilations) to low arousal positive stimuli (e.g., images of babies or puppies; e.g., Augst et al., 2014; Brosch, Sander, Pourtois, \& Scherer, 2008). But because arousal is thought to be the driving force behind attentional biases to emotional stimuli (Mather \& Sutherland, 2011; Padmala et al., 2018; Pool et al., 2016; Vogt et al., 2008), mis-matching negative and positive stimuli on arousal presents an obvious confound. When negative and positive emotional stimuli are matched on high subjective ratings of arousal, they tend to elicit similar attentional biases (Grimshaw et al., 2018; Gupta et al., 2016; Most et al., 2007; Padmala et al., 2018). Positive biases are thought to occur rapidly, involuntarily, and at early stages of attentional processing (Pool et al., 2016). Like negative biases, positive biases are larger when the stimuli match the concerns of the individual (e.g., food stimuli in hungry people; heterosexual erotica in heterosexual people;

, suggesting that motivation plays a role in these biases. Furthermore, extreme positive attentional biases are found in those experiencing addiction or substance abuse, especially when the stimuli match the concerns of the individual (Field \& Cox, 2008; Field, Munafò, \& Franken, 2009; Field et al., 2016; Franken, 2003).

Mechanisms of selective attention. Prioritised attention to emotion is a function of biased attentional competition favouring emotional over non-emotional stimuli in the environment. Non-emotional theories of attention provide a good starting point to understand the mechanisms underlying these biases. Attention is a capacity-limited system (Desimone \& Duncan, 1995). Our environment is rich with sensory information, of which only a small subset is relevant to us at any given time. Despite this potentially overwhelming volume of sensory input, we are mostly able to filter out irrelevant information with ease, attending to the most relevant stimuli at any one moment. This remarkable feat of attentional selection is thought to arise via competition between neural representations of different stimuli or locations (Beck \& Kastner, 2009; Desimone \& Duncan, 1995). The strength of these competing representations is influenced by a combination of exogenous mechanisms that automatically direct attention towards physically salient items (i.e., stimuli that are distinctive from their surroundings by virtue of their physical features, sudden motion, or abrupt onsets; Egeth \& Yantis, 1997; Ranganath \& Rainer, 2003; Serences et al., 2005; Theeuwes, 1991, 1994; Wolfe \& Horowitz, 2004; Yantis \& Jonides, 1984); and endogenous mechanisms that voluntarily guide attention towards goal-relevant stimuli (Beck \& Kastner, 2009; Folk, Remington, \& Johnston, 1992; Noudoost, Chang, Steinmetz, \& Moore, 2010; Posner, 1980; Theeuwes, 1991). Exogenous and endogenous attentional influences are supported by 


\section{Motivation and Emotional Distraction}

dissociable frontoparietal networks, with exogenous attention being mediated by right hemisphere ventral frontoparietal regions such as the ventral frontal cortex and temporoparietal junction; and endogenous attention being mediated by dorsal frontoparietal regions such as the intraparietal sulcus and frontal eye fields (Corbetta, Patel, \& Shulman, 2008; Corbetta \& Shulman, 2002; Peelen, Heslenfeld, \& Theeuwes, 2004).

Traditional models have focussed on the influence of exogenous and endogenous signals on attentional selection (Corbetta \& Shulman, 2002; Desimone \& Duncan, 1995). But recent evidence shows that items that have been selected for attention in the past are also prioritised via a separate mechanism (Awh, Belopolsky, \& Theeuwes, 2012; Belopolsky, 2015; Failing \& Theeuwes, 2018). Although items that have been attended to previously are often relevant to current goals, even when they are not currently goal-relevant (or perceptually salient) they can still capture attention, showing that the selection history signal is dissociable from exogenous and endogenous mechanisms (Awh et al., 2012). Furthermore, neutral stimuli previously associated with monetary reward (Anderson, 2013; Anderson, Laurent, \& Yantis, 2011; Anderson \& Yantis, 2013; Hickey, Chelazzi, \& Theeuwes, 2010; Hickey \& van Zoest, 2012; Le Pelley, Pearson, Griffiths, \& Beesley, 2015; Pearson, Donkin, Tran, Most, \& Pelley, 2015) or threat of shock (Fox, Yates, \& Ashwin, 2012; Hopkins, Helmstetter, \& Hannula, 2016; Schmidt et al., 2015; Yates et al., 2010) receive prioritised attention, even when attending to the stimulus is now counterproductive (Hickey et al., 2010; Watson, Pearson, Wiers, \& Le Pelley, 2019). Remarkably, even if attending to the rewardassociated stimulus has never been beneficial (i.e., the stimulus appeared as a distractor that was presented on trials with high reward), attention is reflexively drawn to the stimulus (Le Pelley et al., 2015; Pearson et al., 2015; Watson et al., 2019).

Thus, reward- and threat-associated stimuli appear to capture attention involuntarily and relatively automatically, independently of current goals or physical salience. It has recently been suggested that these reward and threat associated biases are supported by the same mechanism as selection history biases (Failing \& Theeuwes, 2018), however further evidence is needed to determine whether that is the case. The neural systems underlying the influence of selection history signals on selective attention have yet to be thoroughly investigated, but are thought to involve dopaminergic modulation (Failing \& Theeuwes, 2018). Salience, goal-relevance, and selection history signals then converge on a priority map (Awh et al., 2012; Belopolsky, 2015; Fecteau \& Munoz, 2006; Itti \& Koch, 2000; Theeuwes, 2010) to determine what we attend to at any one moment. These signals interact to enhance the neural representation of objects or locations in visual cortex, with the most relevant 
Motivation and Emotional Distraction

stimuli gaining priority for attentional selection (Beck \& Kastner, 2009; Desimone \& Duncan, 1995). This neural competition has been observed in single-cell studies in monkeys (Reynolds, Chelazzi, \& Desimone, 1999) and in functional Magnetic Resonance Imaging (fMRI) studies in humans (Beck \& Kastner, 2005, 2009; Kastner \& Ungerleider, 2001; Kastner, Weerd, Desimone, \& Ungerleider, 1998).

Prioritised attention to emotional stimuli can occur via all three of these types of mechanisms. First, emotional value has long been thought to operate similarly to physical salience, automatically capturing attention via exogenous mechanisms (Carretié, 2014; Dolan, 2002; Mulckhuyse, 2018; Öhman et al., 2001; Pourtois et al., 2013; Vuilleumier, 2005, 2015). For example, emotional stimuli attract attention to a greater extent than neutral stimuli, even when they are not relevant to current goals (e.g., Vuilleumier, Armony, Driver, \& Dolan, 2001). Second, emotional stimuli are often relevant to our goals, and our attention can be guided to them via endogenous, top-down mechanisms (Brown, Berggren, \& Forster, 2018; Mohanty, Egner, Monti, \& Mesulam, 2009; Mohanty \& Sussman, 2013; Sussman, Jin, $\&$ Mohanty, 2016). For example, attentional biases to emotional information are modulated by current motivational state (e.g., hunger, Mohanty, Gitelman, Small, \& Mesulam, 2008; disgust, Vogt, Lozo, Koster, \& Houwer, 2011); by cues that voluntarily guide attention (Mohanty et al., 2009); and by the motivational value of goal-relevant emotional stimuli (Mohanty et al., 2008). Third, emotional stimuli are likely to have been selected for attention in the past, or to have been previously associated with reward or threat, so emotional biases can also be influenced via the selection history mechanism (Anderson, 2013; Awh et al., 2012; Belopolsky, 2015; Schmidt et al., 2015). Whether attentional biases to emotional stimuli can also occur via an emotion-specific mechanism, dissociable from the above three mechanisms, is a topic of debate (Brosch et al., 2011; Mulckhuyse, 2018; Pessoa \& Adolphs, 2010; Pourtois et al., 2013; Vuilleumier, 2005, 2015).

Neural systems supporting attentional biases to emotion. It has been proposed that a subcortical "low road" exists to efficiently process emotional stimuli (LeDoux, 1996; 2000). The low road begins at the superior colliculus - which receives sensory input - and continues via the pulvinar nucleus of the thalamus, onto the amygdala, completely bypassing cortical regions. The low road is thought to result in "quick and dirty", automatic, unconscious, processing of emotional stimuli (LeDoux, 1996; 2000). The idea of a subcortical low road is intuitively appealing; it would allow us to adaptively respond quickly to threats. However, anatomical and functional evidence for this low road in primates, including humans, is lacking (Pessoa \& Adolphs, 2010). But even if the subcortical low road 


\section{Motivation and Emotional Distraction}

does not exist in humans, the amygdala is still well placed to influence a host of cognitive processes. The amygdala receives rich sensory inputs, and in turn projects directly and indirectly to many cortical and subcortical regions, placing it in an ideal anatomical position for influencing perception and attention (Amaral, Behniea, \& Kelly, 2003; Holland \& Gallagher, 1999). For example, the multiple waves model proposes that rather than a purely subcortical low road, the amygdala and pulvinar have reciprocal connections to and from sensory cortices and frontoparietal cortical regions that influence emotion processing (Pessoa $\&$ Adolphs, 2010). Moreover, Pessoa proposes that amygdala activation does not specifically index emotional value, but stimulus relevance, which encompasses salience, biological significance, novelty, and emotional value. Within the multiple waves model, the amygdala plays a modulatory role in allocating attentional resources to behaviourally relevant stimuli (Pessoa \& Adolphs, 2010).

In contrast to the notion that the amygdala is specialised for relevance (rather than specifically for emotion), and in line with the idea of an emotion-specific attentional mechanism, Pourtois et al. (2013) proposed a framework for understanding how emotional signals influence selective attention, called the Multiple Attention Gain Control (MAGiC) model. According to this model, the amygdala generates a signal that amplifies the "gain" (or neural representation) of emotional stimuli in sensory cortices, via a separate, emotionspecific, mechanism, separate from exogenous, endogenous and selection history mechanisms (Pourtois et al., 2013; Vuilleumier, 2015). Within the MAGiC framework, emotional value enhances perception and attention to emotional stimuli relative to neutral stimuli via early activation of the amygdala, which then influences sensory processing in visual cortex, and interacts with frontoparietal attentional control networks to further influence attention (Pourtois et al., 2013). Evidence for enhanced neural representation of emotional (especially threat) stimuli relative to neutral stimuli in sensory cortices has been observed in PET and fMRI studies (Lane et al., 1998; Pessoa, Kastner, \& Ungerleider, 2002; Sabatinelli, Bradley, Fitzsimmons, \& Lang, 2005; Surguladze et al., 2003; Vuilleumier et al., 2001). Additionally, patients with amygdala damage do not show the usual enhanced neural response to emotional faces in visual cortex

. Enhanced activation in sensory cortices has been linked to upregulation of perception and attention to emotional stimuli (Bocanegra \& Zeelenberg, 2009; Brosch et al., 2010; Phelps, 2006). Moreover, findings from a dot probe study (Brosch, Pourtois, Sander, \& Vuilleumier, 2011) showed that the influence of salient exogenous cues, endogenous arrow cues, and emotional cues, on attention are additive. Furthermore, exogenous and emotional 


\section{Motivation and Emotional Distraction}

cues influenced attention with different timecourses, as demonstrated by dissociable electrophysiological indices, suggesting that they are modulated by separable neural systems. It is thought that this emotional gain control mechanism then converges on attentional and sensory neural pathways along with exogenous, endogenous, and selection history signals (Pourtois et al., 2013; Vuilleumier, 2015). Ultimately, attentional selection is determined by the additive and interactive influence of all four of these mechanisms.

Event-related potentials (ERPs) shed light on the timecourse by which emotion influences attention. ERPs are temporally-sensitive changes in voltage due to summations of neural activity which are time-locked to events (e.g., presentation of an emotional stimulus), measured using electroencephalography (EEG; i.e., electrodes placed on the scalp). ERPs are defined in terms of whether they reflect negative or positive voltage changes, by their timing, and by their location on the scalp (Luck \& Kappenman, 2011; Luck, Woodman, \& Vogel, 2000). Insights into the timing of emotional biasing of attention have been revealed by emotional modulations of early and late ERP components (e.g., the C1, P1, N1, the Early Posterior Negativity or EPN, and the Late Positive Potential or LPP; Hajcak, Dunning, \& Foti, 2009; Hajcak, MacNamara, Foti, Ferri, \& Keil, 2013; Krolak-Salmon, Fischer, Vighetto, \& Mauguière, 2001; Olofsson, Nordin, Sequeira, \& Polich, 2008; Schupp, Flaisch, Stockburger, \& Junghöfer, 2006; Wiens, Sand, \& Olofsson, 2011). For example, emotional modulation of the $\mathrm{C} 1$ begins remarkably early $(80 \mathrm{~ms}$ post-stimulus, generated in striate cortex), and is thought to be independent from exogenous and endogenous influences on attentional selection, providing neural evidence for an early, emotion-specific attention mechanism (Pourtois et al., 2004). Other early components are thought to reflect enhanced perception of, and facilitated attention to, emotional content (e.g., the EPN, Schupp et al., 2006; and the P1, Pourtois et al., 2004). The LPP is a later component that is modulated by emotion, thought to index motivational relevance (Schupp et al., 2000). Thus, ERP studies reveal that emotional content modulates both early perceptual stages, and later elaborative stages of processing.

Automaticity of attentional biases to emotion. In the above section I reviewed extensive evidence that emotional stimuli are prioritised for our perception and attention. But a matter of debate has been whether emotional stimuli capture attention automatically, without intention or attentional resources. Automaticity has been defined in different ways, but "strong" automaticity posits that processing emotional stimuli is involuntary and does not require capacity-limited attentional resources (Pessoa, 2005). "Weak" automaticity, on the other hand, posits that we process emotional stimuli involuntarily (i.e., when they are not 


\section{Motivation and Emotional Distraction}

relevant to current goals), but that doing so does require attentional resources (Pessoa, 2005). At one time, prioritisation of emotional processing was thought to be strongly automatic (Anderson, Christoff, Panitz, Rosa, \& Gabrieli, 2003; Öhman et al., 2001; Vuilleumier et al., 2001). But more recent evidence suggests that processing of goal-irrelevant emotional distractors requires attention, and is therefore weakly automatic (Pessoa, 2005, 2009; Pessoa et al., 2002; Pessoa \& Ungerleider, 2004). Load theory (Lavie, 2005; Lavie, Hirst, de Fockert, \& Viding, 2004) provides predictions that can be used to test whether processing goalirrelevant emotional stimuli is automatic, or whether it requires attention. Load theory posits that distractor processing depends on availability of capacity-limited, common-pool, cognitive resources. If a task is low in perceptual load (i.e., it is perceptually simple), attentional resources are available, and so resources "spill over" to process distractors; whereas if a task is high in perceptual load (i.e., it is perceptually more complex), attentional resources are not available to process distractors. Therefore, distraction will not occur under conditions of high perceptual load. If emotional distractor processing is strongly automatic (i.e., does not require attention), then increasing perceptual load should not influence emotional distraction. However, increasing the perceptual complexity of tasks does reduce attention to goal-irrelevant emotional distractors (Erthal et al., 2005; Fox et al., 2012; Lichtenstein-Vidne, Henik, \& Safadi, 2012; Okon-Singer et al., 2007; Pessoa et al., 2002; Shafer et al., 2012; Van Dillen, Heslenfeld, \& Koole, 2009; Wiens et al., 2011; Yates et al., 2010). Thus, according to the definitions above, attentional biases to emotion are weakly, but not strongly, automatic. We do tend to process goal-irrelevant emotional distractors, but this requires attentional resources.

If attention to emotional stimuli is automatic, it should also be immune to manipulations of cognitive control. Cohen, Henik and Mor (2010) and Cohen, Moyal and Henik (2015) demonstrated that implementation of cognitive control in a flanker task does reduce processing of negative emotional content. In a flanker task, participants respond to the identity of a centrally-presented target symbol that is flanked on either side by either congruent (the same) or incongruent (different) distractor symbols. Cognitive control processes are recruited to resolve the conflict created by the incongruent distractor symbols, which can then facilitate performance on the next trial via a process of conflict adaptation. Following each trial, a negative, neutral, or scrambled distractor image was presented. Negative images impaired performance relative to neutral and scrambled images. But after experiencing incongruent trials, negative images no longer impaired performance (Cohen et al., 2011), and pupil dilation in response to the negative images - an index of emotional 
Motivation and Emotional Distraction

arousal - was reduced (Cohen et al., 2015). Thus, recruitment of cognitive control can modulate distraction by negative emotional stimuli, suggesting that attention to emotional content is not automatic.

\section{Cognitive control and the Dual Mechanisms of Control framework}

To understand how we might be able to control our attention to ignore potent, goalirrelevant emotional distractors, it is useful to examine how we control attention in nonemotional contexts. Much of the cognitive control literature examines how we detect and resolve conflict. Conflict can be at the level of direct competition between responses (or between a response versus no response), or can be at the stimulus level. For example, in a traditional Stroop task (Stroop, 1935), on invalid trials (e.g., "red" appears in green font), there is response-level conflict between the correct response of naming the colour, and the incorrect response evoked by reading the word. There is also stimulus-level conflict between processing the colour and reading the word. In an emotional Stroop task however, the semantic meaning of the word (e.g., "death" in green font) is distracting (which creates stimulus conflict), but it does not create direct response competition (because "death" is not a response option). Distraction therefore involves stimulus conflict, but not response conflict. However, much of the literature on the influence of motivation on cognitive control comes from tasks that index response conflict, which I will describe in the section on The influence of motivation on cognitive control, below. Distraction arises when there is stimulus conflict between goal-relevant information and goal-irrelevant distractors.

Cognitive control refers to a constellation of cognitive processes that allow us to direct our attention towards task-relevant information, while ignoring task-irrelevant information (Cohen, 2017; Gratton et al., 2018; Miller, 2000; Posner \& Snyder, 1975; Shiffrin \& Schneider, 1977). We make reflexive (automatic) and voluntary adjustments to cognitive control depending on stimuli and context. For example, conflict (e.g., an incongruent Stroop trial) automatically facilitates cognitive control on the subsequent trial (Botvinick, Braver, Barch, Carter, \& Cohen, 2001), via a process known as conflict adaptation. In addition to these dynamic changes to control, increasing the frequency of conflict (i.e., increasing the proportion of incongruent Stroop trials, Gratton, Coles, \& Donchin, 1992; or distractors, Forster \& Lavie, 2008) facilitates sustained adjustments to control (Bugg \& Crump, 2012). This sustained upregulation of control is influenced by the expectation that a distractor is likely to occur (Forster \& Lavie, 2008), but the extent to which this change in control strategy is explicitly voluntary is unclear (Bugg, 2012). 
Motivation and Emotional Distraction

Ignoring distractors is an important aspect of cognitive control (Geng, 2014). According to the influential Dual Mechanisms of Control (DMC) framework, we flexibly shift between two modes of control to resolve stimulus or response conflict (Braver, 2012; Braver et al., 2007), and ignore distractions (Geng, 2014). It is thought that we usually operate using a default strategy of reactive control, a collection of control mechanisms that act to detect and resolve conflict or distraction after it has occurred. But sometimes we shift to using more effective proactive control, a collection of control mechanisms that act to anticipate and prevent conflict or distraction before it occurs. The terms "reactive" and "proactive" here refer to the timing of implementation of control, and not to specific mechanisms per se. Reactive mechanisms could include facilitation of conflict detection, or speeding of disengagement from distractors; whereas proactive mechanisms could include, for example, anticipatory enhancement of target representations, or suppression of distractor location or features. Neurally, proactive control recruits lateral prefrontal cortex (PFC) to actively maintain representations of goals (Braver, 2012; Braver, Paxton, Locke, \& Barch, 2009). This activation can be sustained over time, or dynamically implemented before stimulus-onset. Proactive control can be indexed by modulation of ERP components (Czernochowski, 2015), suppression of EEG alpha oscillations (Murphy et al., 2018), and increased pupil dilation (Chatham, Frank, \& Munakata, 2009; Chevalier, Martis, Curran, \& Munakata, 2015; Chiew \& Braver, 2013, 2014) in anticipation of conflict or distraction. Reactive control also recruits lateral PFC, but does so after a distractor has appeared (Braver, 2012; Braver et al., 2009). Reactive control can be indexed by modulation of ERP components (Czernochowski, 2015), and increased pupil dilation (Chatham et al., 2009; Chevalier et al., 2015; Chiew \& Braver, 2013, 2014), after conflict or a distractor has occurred.

If it is so effective, why do we not use proactive control as our default strategy? According to the DMC framework, proactive control requires more mental effort - which is thought to be aversive - so we only make the switch from reactive to proactive control when the perceived benefits of exerting greater effort outweigh the costs (Botvinick \& Braver, 2015; Kool et al., 2017; Shenhav et al., 2017; Yee \& Braver, 2018). Performance-contingent reward is one factor that is known to motivate a shift to proactive control in emotionallyneutral contexts (discussed in more detail below in the section The influence of motivation on cognitive control; for reviews see Botvinick \& Braver, 2015; Yee \& Braver, 2018). This proactive shift is thought to be implemented via connections between reward-processing areas (such as the ventral striatum and orbitofrontal cortex) and cognitive control regions 
Motivation and Emotional Distraction

(such as the anterior cingulate cortex and dorsolateral prefrontal cortex; Botvinick \& Braver, 2015; Pessoa, 2009; Pessoa \& Engelmann, 2010).

\section{The influence of reward on cognitive control}

To understand how motivation enhances cognitive control, it is useful to turn to the reward literature. In order to maximise reward (arguably our most fundamental goal as humans), we need to find a balance between exploiting currently available rewards (stability), while also exploring the environment for other, potentially larger, sources of reward (flexibility; March, 1991). Rewards are not defined by their physical properties, but rather, by the behaviour that they produce. Any stimulus, event, activity, or situation that motivates us to approach it can be defined as a reward (Berridge \& Robinson, 1998; Rolls, 2000; Schultz, 2015). But reward is not a unitary construct, and the influence of reward on behaviour can be divided into three main components: learning, motivation, and affect (Berridge \& Robinson, 1998; Berridge \& Robinson, 2003; Schultz, 2015). Recently, Notebaert and Braem (2016) proposed that these three key components of reward have dissociable influences on cognitive control.

First, the learning component leads to stable exploitation of currently available rewards by encouraging repetition of behaviours that have previously led to reward. The learning component of reward includes instrumental conditioning as reflected in the Law of Effect (Thorndike, 1927) - the notion that if a behaviour results in positive reinforcement, then that behaviour is more likely to occur in the future, because of our fundamental drive to maximise reward. The learning component also includes associative conditioning; neutral stimuli can gain value by virtue of their association with reward (Pavlov \& Anrep, 1927). In contrast to instrumental conditioning, associative conditioning is passive, requiring no response or action. Both the instrumental and associative learning components of reward are thought to increase attention to goal-relevant information that is likely to lead to reward, thereby reducing distraction (Notebaert \& Braem, 2016).

Second, reward elicits approach motivation; sometimes referred to as wanting, incentive salience, or desire (Berridge, 2012; Berridge \& Robinson, 1998). The strength of approach motivation is determined by a stimulus' previously learned associations (e.g., with food, drugs, or sex), and by our current physiological neurobiological state (Berridge, 2012; Zhang, Berridge, Tindell, Smith, \& Aldridge, 2009). For example, in a smoker, the smell of a cigarette (which has previously been associated with nicotine, and has thus gained reward value) may elicit motivation to smoke, which could be modulated by the time that has lapsed since they last had a cigarette, how stressed they are, their level of alcohol intoxication, etc. 


\section{Motivation and Emotional Distraction}

The mesocorticolimbic dopamine circuit includes projections from the ventral tegmental area in the midbrain to the ventral striatum (including the nucleus accumbens); to the PFC; and to the amygdala. There has been much debate as to which component of reward this dopaminergic system subserves. Convincing evidence from animal studies suggests that, rather than encoding pleasure or reward learning, the mesolimbic dopaminergic system underpins approach motivation (Berridge \& Robinson, 1998). The motivational component of reward encourages use of proactive control, enhancing goal-directed behaviour (described in more detail below).

Third, the affective component of reward is our subjective, hedonic, emotional response to the reward itself (i.e., liking or pleasure). The affective component is thought to encourage exploration of the environment. Exploration increases our chances of finding new rewards, but at the cost of making us more distractible from our task (Notebaert \& Braem, 2016). Experiencing a positive emotional state (induced by positive images or randomlydelivered, non-performance-contingent, monetary reward) may increase flexibility, while decreasing stability (Ashby, Isen, \& Turken, 1999; Dreisbach \& Goschke, 2004; Steenbergen, Band, \& Hommel, 2009). The notion that a positive emotional state increases exploration is in line with the Broaden-and-Build theory of emotion (Fredrickson, 2001, 2013), which posits that positive emotion widens the spatial focus of attention, while negative emotion narrows it. It has been suggested that, because experiencing positive emotion signals that things are going well, it indicates that the environment is safe to explore to find new rewards (Carver, 2003). In Notebaert and Braem's (2016) model, positive emotion increases flexibility, task switching, and creativity. However, others have suggested that motivational intensity, and not valence, determines breadth of spatial focus (Gable, Poole, \& HarmonJones, 2015). In this alternative model, positive and negative stimuli that have strong motivational intensity (stimuli that elicit strong approach or withdrawal motivation) narrow the spatial attentional focus, whereas those with weaker motivational intensity broaden the spatial attentional focus.

According to Notebaert and Braem's (2016) model, rewards elicit motivation and positive emotion, which may have opposing influences on cognitive control: with motivation enhancing proactive control, reducing distraction; and positive emotion reducing proactive control, increasing distraction. But empirical findings are mixed regarding whether positive emotion does indeed reduce proactive control. Although some studies find that positive emotion induces flexibility (Chiew \& Braver, 2011; Dreisbach \& Goschke, 2004) at the expense of task performance, other studies do not. One way to examine the influence of the 


\section{Motivation and Emotional Distraction}

positive emotion component of reward, independently of the motivational component, is by randomly delivering reward (not contingent on task performance). For example, Fröber \& Dreisbach (2014) found that performance-contingent reward enhanced proactive control. In contrast, both randomly-delivered reward, and induction of positive emotion, reduced proactive control, and increased reactive control. Thus, under some conditions, the positive affect component of reward could actually counteract the beneficial influence of performance-contingent reward on proactive control. However, Chiew and Braver (2014) also examined the influence of performance-contingent reward versus positive emotion induction on cognitive control. Like Fröber and Dreisbach (2014), they found that performance-contingent reward strongly encouraged proactive control. But, in contrast to Fröber and Dreisbach, they found that positive emotion encouraged a slight shift to proactive control. Thus, findings are mixed regarding the influence of positive emotion on proactive control. Importantly though, the beneficial influence of performance-contingent reward on proactive control does not appear to be undone by the influence of the positive emotion component of reward (Chiew \& Braver, 2011).

\section{The influence of motivation on cognitive control}

When we are motivated, we tend to find it easier to stay on task. In the lab, motivation is often manipulated with availability of performance-contingent reward. Although motivation can affect behaviour by a general increase in arousal, facilitating performance (e.g., by speeding motor responses), research over the past decade has shown that rewards also enhance performance via more specific effects on cognition. Strong evidence suggests that motivation elicits a shift to proactive control to improve performance (see Botvinick \& Braver, 2015; Yee \& Braver, 2018; and below, for reviews). Connections between striatum and PFC are a likely neural correlate for the influence of reward on proactive control (Botvinick \& Braver, 2015; Pessoa, 2015). Below I describe the literature on the benefit of motivation on specific control processes when faced with emotionally-neutral conflicts or distractors. These control processes include working memory, attention, and mechanisms that work to resolve conflict and ignore distractions.

Rewards are often provided in the form of points, that usually represent monetary earnings, but can also be primary reinforcers such as delivery of sweet liquid (e.g., Chiew \& Braver, 2016). Reward can be provided over entire blocks of trials (e.g., Locke \& Braver, 2008); alternatively, reward availability can be signalled by cues that are provided before a target stimulus (e.g., Padmala \& Pessoa, 2011); or can be signalled by a property of the target stimulus itself (e.g., Boehler, Schevernels, Hopf, Stoppel, \& Krebs, 2014; Hopf et al., 2015; 
Motivation and Emotional Distraction

Krebs, Boehler, \& Woldorff, 2010); or can be signalled after a response is made (e.g., Stürmer, 2011). Blocked and cued reward manipulations likely influence cognitive control via anticipatory, proactive processes, because they allow people to predict upcoming availability of reward. In contrast, reward availability signalled by the target stimulus could facilitate reactive control processes. Reward availability signalled by the target stimulus, or after response, do not allow for enhancements of anticipatory control on a trial-by-trial basis. However, these post-stimulus reward signals may elicit more sustained adjustments in proactive control across the entire task, because they signal that rewards are available.

Much of the research on the benefit of motivation on control has used tasks that measure response conflict. For example, the AX-CPT (a continuous performance task; Barch et al., 1997) is often used to examine the influence of motivation on reactive and proactive control of response conflict. In the AX-CPT, participants are instructed to respond only when a specific cue stimulus (an A) is followed by a specific target stimulus (an X). This occurs on the majority of trials (AX trials), but sometimes a different letter is presented as the cue (e.g., a B), or as the target (e.g., a Y), and the response must be withheld. Patterns of response (and inhibiting response) can index dominance of proactive and reactive modes of control. Under a proactive strategy, performance improves on AX and BX trials, because in both cases the cue accurately predicts the correct response (which is to respond on AX trials, and to inhibit a response on BX trials). However, proactive control can also result in more errors on AY trials. Under a proactive strategy, a response is prepared on seeing the A, because an A often indicates that a response is required. It is then more difficult to withhold the response on seeing the Y (which signals inhibition of response). In contrast, under a reactive control strategy, no response is prepared on seeing the cue, and so performance on AY trials improves. On BX trials though, reactive control results in more errors (i.e., failure to withhold a response), because the $\mathrm{X}$ is associated with responding on $\mathrm{AX}$ trials. Thus we can determine whether any manipulation shifts the implementation of control to a proactive mode (by increasing AY errors and decreasing BX errors) or a reactive mode (by decreasing AY errors and increasing BX errors).

The beneficial influence of reward on resolving response conflict has been repeatedly demonstrated (Boehler et al., 2014; Chiew \& Braver, 2013, 2014; Hefer \& Dreisbach, 2016, 2017; Locke \& Braver, 2008). For example, in an fMRI study with a blocked reward manipulation, Locke and Braver (2008) had participants complete the AX-CPT.

Performance-contingent reward sped up responses, and increased errors only on AY trials, consistent with a shift to proactive control. Across the reward block, enhanced activation was 
Motivation and Emotional Distraction

observed in right lateralised frontoparietal attentional control regions. Findings are therefore consistent with motivation eliciting a sustained proactive control strategy to resolve response conflict.

Chiew and Braver $(2013,2014)$ also utilised an AX-CPT to examine the influence of blocked and trial-by-trial reward on cognitive control. In a baseline block, no reward was available; in reward blocks, whether reward was available was signalled by a cue at the start of each trial. They found that reward sped up responses, as well as increasing errors on AY trials, and decreasing errors on BX trials; this pattern is again consistent with motivation eliciting a shift from reactive to proactive control. Chiew and Braver $(2013,2014)$ also measured pupil dilation throughout the task as a measure of cognitive effort (van der Wel \& van Steenbergen, 2018). Cognitive effort can be indexed phasically (dynamic increases in pupil dilation, baselined to a time period immediately prior to a trial); and tonically (sustained increases in dilation over entire blocks of trials). Pupil findings were consistent with both dynamic shifts to proactive control on reward trials, and sustained use of proactive control across blocks in which reward was available (see Chapter 3 of this thesis for further explanation of pupillometry as an index of the timecourse of control). Thus, reward facilitates performance on response conflict tasks by upregulating use of dynamic and sustained proactive control mechanisms.

An important component of proactive control is maintenance of goals in working memory. Reward has beneficial effects on working memory. In an fMRI study, Jimura, Locke and Braver (2010) participants completed a working memory task in a reward and a non-reward context. In the reward context, reward value (no reward, low, and high) varied on a trial-by-trial basis, which was indicated by a cue initiating the trial. The degree to which participants sped up responses in the reward context positively correlated with scores on an individual difference measure of reward sensitivity. Activation of lateral PFC was enhanced in the reward context, consistent with its role in the proactive maintenance of goals in working memory. Furthermore, reward shifted the timecourse of lateral PFC activation; early activation increased, while later activation decreased, consistent with a shift to greater proactive control and less reliance on reactive control. Thus, motivation can enhance performance by enhancing proactive working memory mechanisms.

Motivation also benefits performance on attentional tasks that involve attending to goal-relevant information while ignoring goal-irrelevant information. In a flanker task, the extent to which incongruent distractors interfere with performance is an index of both response and stimulus conflict. Performance improves on flanker tasks when rewards are 
Motivation and Emotional Distraction

offered across entire blocks of trials (Dambacher, Hübner, \& Schlösser, 2011; Hübner \& Schlösser, 2010) or on certain trials within blocks (Yamaguchi \& Nishimura, 2018). Similar benefits of reward on performance have been shown in a traditional Stroop task (van den Berg, Krebs, Lorist, \& Woldorff, 2014). Importantly, cueing availability of reward on an upcoming trial increased an anticipatory ERP component (the contingent negative variation), and suppressed anticipatory oscillatory alpha activity (thought to reflect enhanced attentional preparation). The upregulation of these electrophysiological indices of proactive control predicted faster responses and reduced interference on incongruent Stroop trials. Thus, motivation enhanced proactive control processes to enhance attention to goal-relevant information and reduce attention to goal-irrelevant information.

In line with the above findings, in an fMRI study (Padmala \& Pessoa, 2011) had participants complete a picture-word Stroop-like task in which they responded to whether an image was a building or a house, while attempting to ignore a superimposed word that could be congruent or incongruent with the image (BLDING or HOUSE), or neutral (XXXXX). Like flanker and traditional Stroop tasks, this task creates conflict at both the stimulus and response levels. On each trial a cue indicated whether reward was available or not, with reward and non-reward trials intermixed randomly. Reward reduced interference on incongruent trials, and reduced facilitation on congruent trials, suggesting enhanced filtering of the distractor word's meaning. During presentation of reward cues, subcortical reward processing and cortical frontoparietal regions were activated. Following the stimulus presentation, interference-related activation of medial PFC (including anterior cingulate cortex) and visual processing regions was reduced on reward trials. Furthermore, enhanced neural activation to the reward cue in frontoparietal attentional control regions was correlated with reduced interference-related activation of medial PFC during target processing, consistent with reward enhancing proactive control; and thereby reducing the need for reactive control. Etzel, Cole, Zacks, Kay and Braver (2016) recently had participants complete a cued task-switching picture-word Stroop task under conditions of no reward, and cued trial-by-trial reward. Etzel and colleagues demonstrated (using multivariate pattern analysis in fMRI) that reward enhances cognitive control by improving the discriminability of goal-relevant information in frontoparietal attentional control regions. Collectively, these findings show the benefit of motivation on proactive mechanisms that work to enhance attention to goal-relevant information at the expense of goal-irrelevant information.

Turning to the influence of motivation on more specific attentional processes, performance-contingent reward sharpens visual attention in both exogenous and endogenous 
Motivation and Emotional Distraction

cueing tasks. In the studies described below, motivation was manipulated with availability of performance-contingent monetary reward across entire blocks of trials. In two fMRI studies, (Engelmann, Damaraju, Padmala, \& Pessoa, 2009; Small et al., 2005) participants completed an endogenous cueing task. Perceptual sensitivity to detect a target was enhanced as a function of reward value. Activation of reward processing regions, frontoparietal attentional control regions, and visual processing regions increased parametrically with reward value. Engelmann and colleagues (2009) had participants complete an exogenous cueing task with peripheral cues validly indicating target location on $70 \%$ of trials. Again, perceptual sensitivity improved as a function of reward value. The above studies show that motivation sharpens attention via interactions between reward processing regions and the frontoparietal attentional control network. Further electrophysiological evidence comes from a study by Sawaki, Luck and Raymond (2015) who had participants complete a visual search task with cued low and high reward trials. On high reward trials, during anticipation of the stimulus display, oscillatory EEG alpha activity was suppressed (a marker of attentional preparation). The subsequent post-stimulus N2pc component was reduced on high reward trials. These findings are consistent with motivation eliciting enhanced proactive attentional preparation, reducing the effort required to perform the attentional task.

\section{The influence of motivation on emotional distraction}

Despite a decade of research demonstrating the beneficial influence of reward on cognitive control in non-emotional contexts, it is not yet known whether motivation can also aid control of conflict by irrelevant emotional distractors. Oliveira et al. (2013) proposed that the extent to which emotional distraction can be controlled might be dependent on a push-pull relationship between the level of task motivation and the motivational relevance of the distractors, both of which are modulated by individual differences (e.g., anxiety, reward sensitivity, personal relevance). All experiments in this thesis examine whether performancecontingent reward reduces distraction from positive and negative stimuli, relative to distraction from neutral stimuli. Within the DMC framework, motivation induces a shift to using more effortful but more effective proactive control to resolve conflict and ignore distractions (Botvinick \& Braver, 2015; Yee \& Braver, 2018). However, this idea has almost exclusively been tested in non-emotional contexts, with emotionally-neutral distractors. Here I ask, does this shift to proactive control also occur when distractors are emotional?

There is reason to think that emotional distractions might be controlled by different mechanisms to neutral distractions. Given that attentional biases to emotion are underpinned by multiple mechanisms of attentional selection (i.e., exogenous, endogenous and selection 


\section{Motivation and Emotional Distraction}

history mechanisms; as described in the section Attentional biases to emotional information, above; Awh et al., 2012; Brosch et al., 2011; Carretié, 2014; Yiend, 2010), and possibly even by an emotion-specific mechanism (Pourtois et al., 2013; Vuilleumier, 2015), it follows that emotional and neutral distractions may also be controlled via different mechanisms. Two main hypotheses present themselves, with opposing predictions. The first is that motivation will more effectively enhance control of neutral than emotional distraction. Emotional stimuli are prioritised so heavily for our attention (Yiend, 2010), capturing attention involuntarily (e.g., Okon-Singer et al., 2007). It is therefore reasonable to think that emotional distractions may be more difficult to ignore than neutral distractions, even when we are motivated to ignore them. Additionally, processing high arousal emotional content uses capacity-limited cognitive resources that are also recruited for cognitive control (Pessoa, 2009). If cognitive resources required to control emotional distractors are partly consumed by attending to their content, then control may be less effective, and other control mechanisms may need to be recruited. Note that (as explained in the section The influence of motivation on cognitive control, above) proactive and reactive control are not mechanisms themselves, but refer to constellations of control mechanisms that are implemented before, or after, a distractor appears. Thus, emotional and neutral distraction could both be controlled by proactive, but different, mechanisms. Alternatively, neutral distraction could be controlled proactively, and emotional distraction reactively. Attention is biased toward biologically-relevant emotional stimuli because they are important for survival and reproduction (LeDoux, 2012; Rolls 2000). Therefore, it might be adaptive for them to always capture our attention regardless of their relevance to current goals, so that we can determine whether they require immediate action, before using reactive processes to disengage from them. Because reactive control is less effective than proactive control, it follows that reward would then reduce emotional distraction to a lesser extent than neutral distraction.

But, the opposite prediction is also plausible; reward might reduce emotional distraction to a greater degree than neutral distraction. We know that reward enhances proactive control of distraction in non-emotional contexts. But because reward has motivational value, it may have an additional influence on emotional distraction. In studies of emotional distraction, participants are instructed to attend to task-relevant neutral stimuli while ignoring task-irrelevant emotional stimuli. But these task instructions do not override our biologically-determined goals of looking out for interesting or important emotional events. Thus, competing goals drive attention in the task (i.e., identify the letter; and look out for emotional distractors). Rewarding participants for attending to the neutral task-relevant 


\section{Motivation and Emotional Distraction}

stimuli should increase their motivational value, allowing them to more easily compete with the distractors for our goal-driven attention. Because the emotional distractors have greater motivational value than the neutral distractors, reward should alter this goal-driven competition to a greater extent for emotional than neutral distraction.

Despite both theoretical and practical relevance, empirical evidence on the effects of motivation on control of emotional distraction is scarce. Recent studies, however, provide some support for proactive control of negative emotional distraction under reward. Padmala and colleagues (Padmala \& Pessoa, 2014; Padmala et al., 2017) had participants ignore a centrally-presented distractor image while judging the orientation of two flanking lines. Participants were slower when negative compared to neutral distractors were presented, that is, they were distracted by the emotional content. On some trials, a pre-trial reward cue appeared, indicating availability of monetary reward for fast and accurate performance on the upcoming trial. Reward sped up responses, and also eliminated slowing on negative relative to neutral distractor trials. fMRI findings showed that participants with greater ventral striatum activation during the reward cue phase showed a greater benefit from reward on a behavioural measure of emotional distraction (Padmala et al., 2017). Furthermore, there was stronger functional connectivity between ventral striatum and frontal and parietal regions involved in attentional control while processing reward compared to non-reward cues. These fMRI findings are consistent with the recruitment of proactive control mechanisms to reduce negative emotional distraction when rewards are signalled. Further support for reduced processing of task-irrelevant emotional content under reward comes from an ERP emotional Stroop study (Wei, Wang, \& Ji, 2016) in which participants responded to the colour of negative and neutral words. The emotional modulation of the P3 ERP component was eliminated on cued-reward trials. Thus motivation can modulate neural and behavioural responses to task-irrelevant emotionally negative stimuli.

Reward can also enhance reactive control of distraction from threat-associated stimuli. Hu, Padmala and Pessoa (2013) paired a colour with shock (eliciting fear conditioning) which then sometimes appeared as the background colour in a subsequent visual discrimination task. In the task, participants responded to whether an image was a building or a house. Performance was worse on trials on which the threat-associated colour appeared (i.e., it was distracting). For some participants, reward was available on trials in which a building appeared; for others, reward was available on trials on which a house appeared. Thus, participants did not have prior information about reward availability on a given trial, therefore reward could not elicit proactive control on reward trials relative to no 
Motivation and Emotional Distraction

reward trials. However, the detrimental impact of the threatening colour on performance (observed on non-reward trials) was eliminated on reward trials, suggesting that reward can upregulate reactive control processes to improve performance and reduce negative emotional distraction.

Although there is some evidence for a beneficial effect of reward on control of negative emotional distraction, much less is known about how reward affects control of positive emotional distraction. An important consideration when predicting the effects of reward on emotional distraction is that the function of emotional stimuli is to prepare the body for action, with positive stimuli guiding us to approach, and negative stimuli to withdraw (Rolls, 2000). Because of these opposing motivational functions, positive and negative distractions could plausibly be controlled in different ways. For example, negative stimuli may be treated as requiring immediate attention and action, because they might signal an impending threat. In contrast, positive stimuli signal potential reward. Although we don't want to miss out on rewards, doing so will usually not immediately harm us, or threaten our survival. Given these differences in the functions of attentional biases to positive and negative stimuli, we might predict that manipulations of control would be more effective for reducing positive than negative distraction.

Somewhat surprisingly, in light of these different functions of positive and negative stimuli, some manipulations of control reduce negative but not positive distraction. For example, using a letter search task with centrally-presented emotional distractor images, Gupta and colleagues (2016) found that increasing the task's perceptual load eliminated distraction from negative, but not positive, images. Similarly, Most and colleagues (2007) reported that pre-cueing the identity of a non-emotional target in a stream of rapidly presented images reduced distraction from negative, but not positive, images. If motivation acts via similar control mechanisms to those elicited by these attentional manipulations, then we might expect reward to attenuate negative but not positive distraction. However, conflicting findings have been reported; some manipulations of control reduce both negative and positive distraction. For example, two recent studies from our lab (Grimshaw et al., 2018; Murphy et al., 2018) showed that increasing distractor frequency reduced distraction from both negative and positive images. Similarly, Kennedy, Newman and Most (2017) presented participants with a stream of rapidly presented images. Sometimes they warned participants about the content of an upcoming emotional distractor that appeared shortly before a nonemotional target. These warnings slightly reduced both positive and negative distraction. 


\section{Motivation and Emotional Distraction}

Whether various manipulations of control affect negative and positive distraction to similar or different degrees may depend on the mechanisms elicited by the manipulation of control.

Another consideration is that performance-contingent reward and positive emotional distractors both elicit approach motivation and subjective positive emotion (Chiew \& Braver, 2011). Processing negative and positive stimuli share some neural systems (e.g., they both activate the amygdala; Pessoa, 2009). But positive stimuli and reward additionally activate dopaminergic midbrain regions such as the ventral striatum (Gable, Poole, \& Harmon-Jones, 2015), and influence opioid and GABA transmission in the nucleus accumbens (Peciña \& Berridge, 2005). Furthermore, although we are biased to attend to both negative and positive stimuli, we may be more motivated to attend to positive than negative emotional distractors, given that processing positive stimuli can be rewarding. Perhaps this motivation to attend to positive stimuli is the reason that positive distraction is not reduced by some manipulations of control (Gupta et al., 2016; Most et al., 2007). Given that performance-contingent reward and positive distractors share this motivational pull, reward might influence control of positive distractors differently to negative ones.

\section{The thesis experiments}

My thesis connects two largely separate lines of research on the influence of motivation on cognitive control in non-emotional contexts on the one hand (e.g., Chiew \& Braver, 2013; Padmala \& Pessoa, 2011); and the influence of non-reward manipulations of control on emotional distraction on the other (Grimshaw et al., 2018; Gupta et al., 2016). In four experiments, I examine the influence of performance-contingent reward on distraction from positive, negative, and neutral task-irrelevant images, to determine whether, and how, motivation influences control of emotional distraction. All experiments use variations on an irrelevant distractor paradigm (Forster \& Lavie, 2008; Gupta et al., 2016) in which participants respond as quickly and accurately as possible to indicate whether a $\mathrm{K}$ or an $\mathrm{N}$ is present within an array of Os. The task is low in perceptual load (Lavie et al., 2004), so there are attentional resources available to process the distractors (Forster \& Lavie, 2008). On 25\% of trials, task-irrelevant distractor images are also presented, and participants are instructed to ignore them. The key dependent variable is distraction, indexed by slowed responses on distractor trials relative to trials on which a scrambled, meaningless image is present (Experiments 1 and 2), or trials on which no distractor is present (Experiments 3 and 4).

Previous work in our lab shows that this relatively low distractor frequency encourages a default reactive mode of control (and therefore large distraction), as there is little advantage to maintaining proactive control when distractors appear rarely (Grimshaw et 
Motivation and Emotional Distraction

al., 2018; Murphy et al., 2018). The images (selected from the International Affective Picture System; IAPS, Lang, Bradley, \& Cuthbert, 2008) are blocked by valence, such that the emotional content of the images is predictable within a block. The negative images depict mutilations, and are rated highly on subjective arousal ratings, and low on subjective valence ratings. The positive images depict erotic scenes of heterosexual couples, and are rated highly on subjective arousal ratings, and highly on subjective valence ratings. The neutral images depict people in everyday scenarios and are rated low on subjective arousal ratings, and moderately on subjective valence ratings. The neutral images match the emotional images on content (i.e., they all contain people), and so have social and biological relevance, but lack emotional valence and arousal.

In all experiments, participants are randomly allocated to either a control group, who perform the task with no performance-contingent reward; or a reward group, who have the opportunity to earn points on each trial for fast and accurate performance (which translates into greater monetary reward in Experiments 1, 2 and 3, but not in Experiment 4). The blocked, between-subjects, reward manipulation was intended to induce a sustained proactive control strategy across the task.

Experiment 1 (Chapter 2) examines the influence of motivation on control of emotional distraction under conditions in which the distractors are presented centrally, within the spatial locus of attention (as in Gupta et al., 2016). Experiment 2 (Chapter 3) replicates Experiment 1, and additionally measures pupil dilation over time - an online measure of cognitive and emotional processing - to test whether motivation elicits a shift to proactive control to ignore emotional distractions, as it does in non-emotional contexts. Given the differences in mechanisms underlying attention to neutral versus emotional distractors, the timecourse of reward-elicited control of emotional and neutral distraction may also differ. The two experiments in Chapter 4 (Experiments 3 and 4) examine the influence of motivation on control of emotional distraction under conditions in which peripherallypresented distractors capture attention away from the centrally-presented targets in order to cause distraction (as in Forster \& Lavie, 2008). In Experiments 1, 2 and 3, motivation was manipulated with the use of performance-contingent monetary reward, which was represented by the accumulation of points based on fast and accurate performance. In Experiment 4), the points did not represent monetary reward, to determine whether nonmonetary reward can motivate a shift to better control of emotional distractions.

Based on current theory on motivation, cognitive control, and emotional biases, reviewed in this chapter, two main alternative predictions present themselves. First, given the 
Motivation and Emotional Distraction

potency of attentional biases to emotion (Yiend, 2010), and the differences between the mechanisms underlying attention to emotional versus neutral stimuli (Pourtois et al., 2013), reward might reduce emotional distraction to a lesser degree than neutral distraction. And if emotional distraction is automatic in the sense that it occurs without intention (e.g., OkonSinger et al., 2007), perhaps it will not be modulated by motivation at all. Alternatively, because emotional stimuli are relevant to our goals of surviving and reproducing, reward might alter the goal-driven attentional competition between the letter targets and the emotional distractors to a greater degree than the neutral distractors; therefore reducing emotional distraction to a greater degree than neutral distraction. Furthermore, I compare the influence of motivation on control of positive versus negative distraction across all experiments. It is possible that reward will influence positive and negative distraction differently, because reward and positive stimuli both elicit motivation and positive emotion. Finally, I consider the influence of reward under conditions in which distractors are presented centrally, within the current spatial locus of attention (Experiments 1 and 2), to conditions in which distractors are presented peripherally, outside the current spatial locus of attention (Experiments 3 and 4). It is possible that reward might influence different attentional mechanisms to control distraction under these different presentation conditions (outlined further in Chapter 4). 
Motivation and Emotional Distraction

Chapter 2. Motivation enhances control of positive and negative emotional distractions.

Portions of this chapter appear in: Walsh, A. T., Carmel, D., Harper, D., \& Grimshaw, G. M. (2018). Motivation enhances control of positive and negative emotional distractions.

Psychonomic Bulletin \& Review, 25(4), 1556-1562. https://doi.org/10.3758/s13423-017-

1414-5

Preprint available at https://osf.io/5kcvu/ and in Appendix A.

Open data, code, materials, and analyses available for the published manuscript available at https://osf.io/qu2d6/.

Open data and code to reproduce analyses for this chapter are available at https://osf.io/vxwd4/

According to the DMC framework, in emotionally-neutral contexts, motivation is thought to reduce distraction by enhancing proactive control (Botvinick \& Braver, 2015; Yee $\&$ Braver, 2018). But our attention is heavily biased toward emotional stimuli, via multiple mechanisms of attentional selection (Awh et al., 2012; Brosch et al., 2011; Mohanty \& Sussman, 2013; Mulckhuyse, 2018; Pourtois et al., 2013; Sussman et al., 2016; Vuilleumier, 2015). We may therefore control our attention to ignore emotional stimuli via different mechanisms than we do for neutral stimuli. It is not yet known whether task motivation can enhance control of positive and negative emotional distractions. In this chapter I describe an experiment that tests whether reward can reduce distraction from task-irrelevant negative, neutral, and positive images.

Findings from two recent studies (Padmala \& Pessoa, 2014; Padmala et al., 2017; described in Chapter 1: General Introduction) showed that providing performancecontingent reward for fast and accurate performance attenuated distraction from negative images. In their task, participants responded to indicate whether two lines that flanked a centrally-presented negative or neutral distractor image were parallel, or not. When no reward was available, performance was impaired for negative relative to neutral distractor trials; but on reward trials this difference was eliminated. Thus, despite attentional biases to negative stimuli, motivation can help us to control negative distraction. Their findings are consistent with predictions from the DMC framework, in which providing reward to elicit motivation is thought to encourage a shift to using proactive control to resolve conflict (e.g., Chiew \& Braver, 2013, 2014), or ignore emotionally-neutral distractions (e.g., Padmala \& Pessoa, 2011). Notably, Padmala and colleagues (Padmala \& Pessoa, 2014; Padmala et al., 2017) were the first to apply predictions from the DMC in the context of ignoring negative 
Motivation and Emotional Distraction

distractors. Here we ${ }^{1}$ examine whether the influence of motivation on proactive control can be extended to situations in which distractors are highly arousing negative and positive images.

Other experimental manipulations of control can reduce distraction from positive and negative images. For example, a recent study from our lab (Grimshaw et al., 2018) used an adapted version of Forster and Lavie's (2008) irrelevant distractor paradigm to examine the influence of distractor frequency on attentional control of emotional distraction. Their participants responded as quickly and accurately as possible to whether they saw a K or an $\mathrm{N}$ in a briefly presented array of o's. On a proportion of trials, positive, negative, or neutral images unpredictably appeared, above or below the central target array. Emotional distractor images captured attention to a greater degree than neutral ones, but only when they were presented rarely (on $25 \%$ of trials), and not when they were presented frequently (on $75 \%$ of trials). In a follow-up study (Murphy et al., 2018), the effect of distractor frequency on emotional distraction replicated, and was accompanied by suppression of anticipatory occipital alpha power -an electrophysiological marker of proactive control. This finding is consistent with a shift to proactive control when the benefits outweigh the costs (Botvinick \& Braver, 2015; Kool et al., 2017; Shenhav et al., 2013; Yee \& Braver, 2018), that is, it is more beneficial to exert the extra effort to use proactive control if we expect distraction to occur frequently.

The current experiment used an adaptation of the paradigm used by Grimshaw and colleagues (2018) to examine the influence of reward on control of emotional distraction. Instead of central letter targets and peripheral distractors (as in Grimshaw et al., 2018; Murphy et al., 2018), our participants completed a letter identification task while attempting to ignore centrally-presented distractor images. The letter targets appeared above and below the images. Presenting distractors centrally creates greater distraction than when they are presented in the periphery (Beck \& Lavie, 2005). Furthermore, central distractors appear within the current spatial locus of attention, so attention must be shifted to the letter targets to perform the task. In contrast, peripheral distractors must capture attention away from the centrally presented targets in order to impair performance. Given these differences in the attentional mechanisms underlying distraction from central versus peripheral images, it is important to determine whether manipulations of control influence distraction in both

\footnotetext{
${ }^{1}$ Although this thesis is my own work, I use "we" when referring to the experiments, to acknowledge the contribution of my coauthors to the research. I use "I" when referring to the thesis as a whole.
} 
Motivation and Emotional Distraction

paradigms (see Chapter 4 for two experiments examining the influence of reward on distraction from peripherally-presented images).

Our paradigm is similar to that of Padmala and colleagues' (Padmala \& Pessoa, 2014; Padmala et al., 2017) in that participants complete a visual task while attempting to ignore centrally-presented distractor images. However, our task allows us to examine the effect of reward on distraction from negative, positive, and neutral images separately. In Padmala and colleagues' task, a negative or neutral distractor image was presented on every trial; their index of negative distraction was therefore slowing on negative relative to neutral image trials. Therefore they were able to determine if reward reduced the extent to which emotional images were more distracting than neutral images, but they could not determine whether reward reduced distraction caused by neutral images themselves. In contrast, in our task, intact distractor images unpredictably appeared on $25 \%$ of trials. Scrambled, meaningless distractor images were presented on the remaining $75 \%$ of trials, creating a baseline condition from which to calculate slowing on intact positive, negative, and neutral distractor trials. Distractor valence was consistent (and thus predictable) within a block. Presenting intact distractors on $25 \%$ of trials creates large distraction effects (Grimshaw et al., 2018), which is thought to reflect use of reactive control (Bugg \& Crump, 2012). Our positive images of erotic couples and negative images of mutilations were matched on similarly high subjective arousal ratings (Lang et al., 2008). In contrast, the neutral images (of people in everyday scenarios) were rated as subjectively low on arousal. Therefore, using our task, it is possible to examine whether the influence of reward on distraction is dependent on distractor arousal (high versus low), or distractor valence (positive versus negative).

There are plausible reasons why emotional distraction might be controlled differently to neutral distraction. In short, emotional stimuli are prioritised for our attention relative to neutral stimuli (e.g., Yiend, 2010), via endogenous, exogenous, and selection history mechanisms (Awh et al., 2012; Brosch et al., 2011; Mohanty \& Sussman, 2013; Mulckhuyse, 2018; Pourtois et al., 2013; Sussman et al., 2016; Vuilleumier, 2015), and possibly even via an emotion-specific mechanism (e.g., Pourtois et al., 2013; Vuilleumier, 2015). Emotional distraction may therefore be more difficult to control than neutral distraction, no matter what the control manipulation. If this is the case, then reward may reduce emotional distraction to a lesser extent than neutral distraction. On the other hand, in emotional contexts, over and above encouraging proactive control (as it does in non-emotional contexts; e.g., Botvinick \& Braver, 2015; Yee \& Braver, 2018), reward might also increase the motivational value of the letter targets, allowing them to better compete with the motivational value of the distractors. 
Motivation and Emotional Distraction

Because emotional stimuli have greater motivational pull than neutral ones, reward might therefore exert a greater benefit for controlling emotional than neutral distraction.

It is not yet known whether motivation enhances control of positive distractions, as it does for negative distractions (Padmala \& Pessoa, 2014; Padmala et al., 2017). Attention is biased to high arousal positive stimuli, even when they are irrelevant to current goals (Pool et al., 2016). But previous findings are mixed as to whether various other (non-reward) manipulations of control influence negative distraction to a greater degree than positive distraction (Gupta, et al., 2016; Most et al., 2007), or to a similar degree (Grimshaw et al., 2018; Kennedy et al., 2017). Thus, whether control is more effective for negative than positive distraction may depend on the type of manipulation that is used to elicit control. Moreover, both reward and positive images elicit both approach motivation and subjective positive emotion, activating appetitive motivational circuits (Bradley et al., 2001; Chiew \& Braver, 2011). Aversive stimuli on the other hand, elicit withdrawal motivation and subjective negative emotion, activating defensive motivational circuits (Bradley et al., 2001). The fact that positive and negative stimuli have these opposing influences on motivation opens up the possibility that reward (which, like positive stimuli, elicits approach motivation) might influence positive distraction differently to negative distraction. Given these conflicting theoretical perspectives and empirical findings, we did not have a specific hypothesis regarding how reward would influence negative versus positive distraction.

In the current experiment, participants were randomly allocated to the reward or control groups, to perform the central distractor task with or without availability of performance-contingent reward. Participants in the reward condition earned points for fast and accurate performance, which translated into greater monetary earnings at the end of the session. Control participants received a fixed monetary amount for participating, which was not dependent on performance. Distraction was indexed as slowing on intact distractor relative to scrambled distractor trials, within a block. We expected emotional images (both positive and negative) to be more distracting than neutral images, reflected in a distractor type (intact versus scrambled) $\times$ distractor valence interaction. We expected reward to speed up performance, reflected by a main effect of reward group on response time. Crucially, if motivation reduces distraction by upregulating cognitive control, we predicted less distraction in the reward group than the control group (a reward group $\times$ distractor type interaction). We had two alternative hypotheses regarding how motivation influences emotional versus neutral distraction, which would be reflected by different reward group $\times$ distractor type $\times$ distractor 
Motivation and Emotional Distraction

valence interactions. First, we predicted less benefit of reward on emotional than neutral distraction, because attention is so biased toward emotional stimuli. Alternatively, if motivation also benefits performance by enhancing the motivational value of the letter targets, allowing them to more effectively compete with the emotional distractors, we predicted that reward would have a greater benefit on emotional versus neutral distraction.

\section{Method}

\section{Participants and sample size determination}

A total of 73 participants were recruited from the community in Wellington, New Zealand, via flyers, emails, and social media posts. Participants were all women, to reduce within-group variance, and to allow for use of images that were carefully calibrated for valence and arousal ratings, which differ across genders. Exclusion criteria were: achieving less than $75 \%$ accuracy overall; less than $60 \%$ accuracy on a single block; or showing distraction greater than 4 standard deviations $(S D s)$ above the mean in any condition. No participants met the exclusion criteria of low accuracy. One participant was excluded for displaying distraction greater than $4 S D$ above the mean for the negative distraction condition in the reward group. The excluded participant was replaced, resulting in 72 participants in the final sample (mean age 22.40; range 18-37 years), who were randomly split into two groups. Control participants understood they would receive a $\$ 15^{2}$ supermarket voucher for participating. Reward participants understood they could earn between $\$ 10$ and $\$ 20$, contingent on performance.

Because of the scarcity of studies examining the influence of reward on emotional distraction, accurately estimating an effect size to conduct a power analysis to determine sample size was not possible. Due to the cost of paying community participants, we decided on the largest sample size our budget allowed: 36 per group. A sensitivity analysis using G*Power (Faul, Erdfelder, Lang, \& Buchner, 2007) determined that a sample size of 36 participants per group would have an $80 \%$ probability of detecting an effect of reward on distraction greater than or equal to $d_{s}=0.700$ (alpha $=.05$, two-tailed).

In all experiments in this thesis, participants spoke fluent English, had normal or corrected-to-normal vision, and reported no current treatment for depression or anxiety. All

\footnotetext{
${ }^{2}$ All dollar amounts throughout this thesis are in New Zealand dollars.
} 
Motivation and Emotional Distraction

experiments in this thesis were approved by the Victoria University of Wellington Human Ethics Committee, and participants gave informed consent before participating. Analyses for each experiment were undertaken after all data were collected.

\section{Materials}

Participants ran in groups of one to four in a dimly-lit room in separate booths; all participants in a session were run in the same experimental condition. The experiment was run using E-Prime 2.0 (Psychology Software Tools, Pittsburgh, PA, USA), on Dell Precision T1700 personal computers with 24 " monitors at $1920 \times 1080$ pixel resolution and a $120 \mathrm{~Hz}$ refresh rate. A chin-rest maintained a viewing distance of $57 \mathrm{~cm}$.

Twelve images for each category (negative, neutral, positive) were drawn from the IAPS (Lang et al., 2008). See Table 1.1 for arousal and valence ratings of the images; and Table S.1 (see Appendix B) for a list of the specific images by IAPS number. These same images were used in all experiments.

Table 1.1 Mean IAPS valence and arousal rating for each valence image set for $\mathrm{men}^{3}$, and for women. The same images were used across all experiments in this thesis. However, only Experiment 2 included men.

\begin{tabular}{ccccc}
\hline & \multicolumn{2}{c}{ Valence Mean } & \multicolumn{2}{c}{ Arousal Mean } \\
& Women & Men & Women & Men \\
\hline Neutral & 5.01 & 4.97 & 3.07 & 3.16 \\
Negative & 1.64 & 1.83 & 6.53 & 6.43 \\
Positive & 6.23 & 7.09 & 6.31 & 6.39 \\
\hline
\end{tabular}

The positive images (scenes of heterosexual erotic couples) and negative images (scenes of body mutilations) were matched on arousal using normative ratings from women, measured on a 9-point Likert scale. The neutral images contained people and therefore had biological and social relevance, but lacked emotional valence, and were rated low in arousal. To create scrambled images, intact images were divided into $36 \times 36$ segments that were randomly recombined in PhotoScape v3.7. Scrambled images thus had the same lower level visual properties as intact distractors; comparing performance on intact vs. scrambled trials therefore estimates distraction due to the images' meaning. All images (intact and scrambled) were matched for luminance using the SHINE MATLAB toolbox (Willenbockel et al., 2010)

\footnotetext{
${ }^{3}$ Note that no men were included in Experiment 1, however the men's ratings are included here as men were included in the sample of Experiment 2, which used the same image sets.
} 
Motivation and Emotional Distraction

Participants completed the Behavioural Activation Scale and the Behavioural

Inhibition Scale (the BIS/BAS; Carver \& White, 1994) at the end of the experimental session. Scores on the BAS Drive were used for exploratory analyses of individual differences in reward sensitivity. However, my experiments were not adequately powered to test for such individual differences, and they were outside the scope of this thesis, so are not reported.

\section{Task and Procedure}

Figure 2.1 illustrates the distraction task.



Figure 2.1. Trial sequence (left) and target display (right) for the central distractor task in Experiment 1. The trial sequence depicts a successful reward trial with a neutral intact distractor image. The target display (right) depicts a scrambled distractor trial. Distractors were presented on $25 \%$ of trials, and were blocked by valence (negative, neutral, positive). Participants in the control condition experienced the same trial sequence, but with no bell sound. For illustrative purposes, images are not to scale; the central part of the display is enlarged.

For all participants, regardless of reward condition, a trial began with a central white fixation cross (jittered duration between 417-833 ms), presented on a black background. The target display comprised a centrally-presented colour image $\left(11^{\circ}\right.$ width $\times 8.26^{\circ}$ height; intact on $25 \%$ of trials, scrambled on $75 \%)$, flanked by six white letters $\left(0.86^{\circ} \times 0.92^{\circ}\right)$; three 
Motivation and Emotional Distraction

located $0.75^{\circ}$ above and three $0.75^{\circ}$ below the image's horizontal edge. Five of the letters were Os and the target letter was either a $\mathrm{K}$ or $\mathrm{N}$.

Participants were instructed to respond as quickly and accurately as possible to indicate whether the target letter $\mathrm{K}$ or $\mathrm{N}$ was present, using keys 1 and 2 (counterbalanced across participants) on the number pad with their dominant hand. They were also instructed to ignore the images. Participants responded during a $1900 \mathrm{~ms}$ response-window from stimulus-offset. Failure to respond was recorded as an error. A $600 \mathrm{~ms}$ blank screen followed the response, followed by visual feedback $(100 \mathrm{~ms})$ : "correct" in green, "incorrect" in red, or "please respond faster" in white (if no response was made within the response-window). A random inter-trial-interval ranged between 207-623 ms. Target letter and location were counterbalanced across trials, and trial order was randomised within blocks.

Participants completed 24 practice trials (six intact distractor trials, and 18 scrambled distractor trials) and a baseline block of 48 trials. The baseline images were 12 intact neutral images of people - a different set to that used in the experimental neutral distractor blocks. Twelve scrambled versions of these images were presented on the remaining 36 trials. The images used in the practice and baseline trials did not appear in the rest of the experiment. The remainder of the experiment was split into two halves (super-blocks) of 192 trials (counterbalanced), to separate the negative and positive blocks. One super-block was split into blocks of negative and neutral distractors; the other super-block was split into blocks of positive and neutral distractors. A three-minute break separated the super-blocks, to limit valence carry-over effects. During the break, participants filled out a sudoku puzzle. Each super-block comprised two emotional and two neutral blocks (separated by self-timed breaks), presented in ABBA order, counterbalanced across participants. Within a block (48 trials; 12 intact, 36 scrambled), all intact images were either negative, neutral, or positive; randomly mixed with scrambled distractor trials, which were scrambled versions of the intact distractor images of the same valence in that block. Each image was presented once in a block, although images were repeated (emotional images twice; neutral images four times) across the experiment to increase trial numbers to obtain stable measures of response times and distraction. This yielded four block orders, which were counterbalanced across participants. Total experiment session time was approximately 50 minutes.

Reward Condition. After the baseline block, participants who were in the reward condition were informed about the potential to earn one point per trial if they were correct and faster than their median RT from the baseline block. Success was indicated by a pleasant 
Motivation and Emotional Distraction

sound (600 ms duration) immediately post-response. Winning points led to achieving levels and reaching a new level increased total earnings by $\$ 2.50$. Participants began on level 1 (total earnings: \$10). Level 2 required 91 points (total earnings: \$12.50). Ninety-one additional points were needed to ascend to each new level, up to level 5 (total earnings: \$20). During each break between blocks, participants' points total and current level appeared above an animation of coins falling into piles (see Figure 2.2), with their mean RT and mean percent accuracy for the preceding block. Participants were encouraged to continue trying to be fast and accurate to get to the next level. A warning to try to keep accuracy high was provided if accuracy fell below 95\% in any block. Reward participants all earned between $\$ 15$ and $\$ 20$ $(M=\$ 16.22)$.



Figure 2.2. A screenshot of the animation of coins presented to reward participants between blocks. The animation shows the coins falling into piles. Current point total and current level are displayed on screen.

Control Condition. Control participants completed the task as above, but were all given a total of $\$ 15$ for completing the experiment, which was not dependent on performance. They received no auditory trial-by-trial feedback, because no reward was available. During the breaks, instead of performance feedback and the coin animation, they saw "please wait...", and instructions to take a break. 
Motivation and Emotional Distraction

RTs faster than $200 \mathrm{~ms}$ were excluded as they were assumed to be anticipatory. This comprised $<0.01 \%$ of trials in each experiment. A high cut-off was not used as the responsewindow was limited to $2000 \mathrm{~ms}$ from stimulus-onset.

All reported effect sizes for Analyses of Variance (ANOVAs) are $\eta_{p}{ }^{2}$ and $\omega^{2}$ (with $\omega^{2}$ being less biased; Lakens, 2013). Welch's tests are used as the default (instead of independent $t$-tests) for between-subjects comparisons as they are robust to violations of heterogeneity of variance (Delacre, Lakens, \& Leys, 2017). Effect sizes are Cohen's $d_{s}$ for between-subject comparisons (the mean difference divided by the pooled standard deviation; which is the square root of the average variance in each condition; (Cohen, 1992; Lakens, 2013), and Cohen's $d_{z}$ for within-subject comparisons (the mean difference divided by the standard deviation of the difference scores; Cohen, 1992; Lakens, 2013). Degrees of freedom are adjusted for violations of sphericity (Greenhouse-Geisser correction) when necessary. Confidence-intervals are $95 \%$ and effects are considered significant if $p<.05$. Predicted marginally significant effects are followed up if $p<.10$. All behavioural analyses were performed in Jamovi (2018). All data and code to reproduce analyses and figures for all experiments are available at https://osf.io/vxwd4/.

\section{Results}

RTs and accuracy for neutral blocks did not differ between the two positive and negative super-blocks (intact and scrambled conditions; $p$ 's > .260), so we collapsed the neutral blocks in the analyses. Mean correct RTs and accuracy for each condition, with comparisons between intact and scrambled conditions, are presented in Table 2.2. RT distraction indices [intact RT - scrambled RT], and accuracy distraction indices [scrambled proportion correct - intact proportion correct] were calculated to follow up on any interactions with distractor type. See Figure 2.3 for mean distraction [intact RT - distractor RT] in each condition. 
Motivation and Emotional Distraction

Table 2.2. Comparisons of mean correct RTs (ms) and accuracy (proportion correct) for scrambled versus intact images (distraction indices in $\mathrm{ms}$ ), for each distractor valence condition, separately for control and reward groups for Experiment 1. SDs are in brackets.

\begin{tabular}{|c|c|c|c|c|c|c|}
\hline \multirow{2}{*}{$\begin{array}{l}\text { Valence } \\
\text { Block }\end{array}$} & \multirow{2}{*}{$\begin{array}{l}\text { Scrambled } \\
\text { Trials }\end{array}$} & \multirow{2}{*}{$\begin{array}{l}\text { Intact } \\
\text { Trials }\end{array}$} & \multirow{2}{*}{ Distraction } & \multirow{2}{*}{$d_{z}$} & \multicolumn{2}{|c|}{$95 \% \mathrm{CI}$} \\
\hline & & & & & Low & Upper \\
\hline \multicolumn{7}{|c|}{$\underline{\text { Response Times }}$} \\
\hline \multicolumn{7}{|c|}{ Control Group } \\
\hline Negative & $614(116)$ & $710(155)$ & $96^{* * *}(72)$ & 0.61 & 72 & 121 \\
\hline Neutral & $618(120)$ & $648(131)$ & $30 * * *(25)$ & 0.21 & 21 & 38 \\
\hline Positive & $624(117)$ & $712(156)$ & $88 * * *(70)$ & 0.54 & 64 & 111 \\
\hline \multicolumn{7}{|c|}{ Reward Group } \\
\hline Negative & $550(80)$ & 603 (119) & $53 * *(60)$ & 0.42 & 33 & 74 \\
\hline Neutral & $551(79)$ & $573(85)$ & $22 * * *(18)$ & 0.26 & 16 & 28 \\
\hline Positive & $554(83)$ & $596(113)$ & $42 * * *(48)$ & 0.34 & 26 & 58 \\
\hline \multicolumn{7}{|c|}{ Proportion Correct } \\
\hline \multicolumn{7}{|c|}{ Control Group } \\
\hline Negative & $.950(.041)$ & $.902(.080)$ & $.048 * * *(.069)$ & 0.70 & .025 & .071 \\
\hline Neutral & $.958(.031)$ & $.942(.048)$ & $.016(.039)$ & 0.35 & .002 & .028 \\
\hline Positive & $.949(.045)$ & $.905(.072)$ & $.044 * *(.075)$ & 0.72 & .019 & .070 \\
\hline \multicolumn{7}{|c|}{ Reward Group } \\
\hline Negative & $.899(.067)$ & $.877(.089)$ & $.022 \quad(.072)$ & .27 & -.002 & .047 \\
\hline Neutral & $.919(.059)$ & $.898(.069)$ & $.021 * *(.044)$ & .32 & .006 & .036 \\
\hline Positive & $.915(.066)$ & $.881(.085)$ & $.034 * *(.070)$ & .44 & .010 & .058 \\
\hline
\end{tabular}

Notes: Distraction is calculated as: [RT intact - RT scrambled] and [proportion-correct scrambledproportion-correct intact]. SDs are in brackets. Effect sizes are Cohen's $d_{z}$ from paired comparisons of intact and scrambled trials within conditions, and asterisks indicate whether the [intact scrambled] difference is significant in that condition. ${ }^{* *} p<.01,{ }^{* * *}<.001 .95 \%$ confidence intervals surround the distraction scores, in ms and proportion correct. $n=36$ per group. 
Motivation and Emotional Distraction

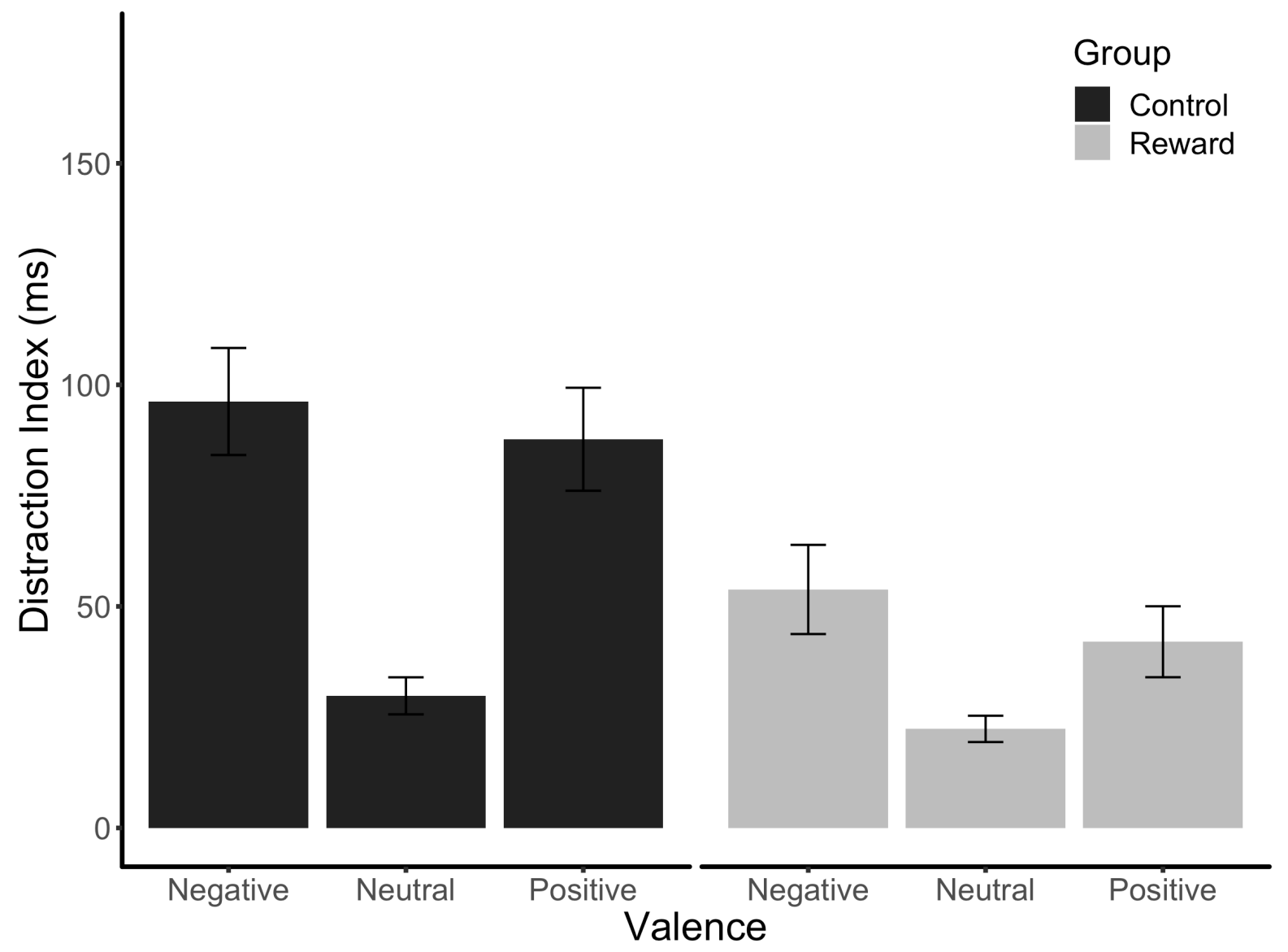

Figure 2.3. Mean behavioural distraction indices (in ms) by reward group and valence for Experiment 1. Greater distraction [RT intact - RT scrambled] was elicited by emotional than by neutral images. Emotional (but not neutral) distraction was attenuated by reward. Error bars represent standard error of the mean.

\section{Response times}

To examine the influence of reward on task performance, correct RTs were analysed in a 2 (reward group: reward, control) $\times 2$ (distractor type: intact, scrambled) $\times 3$ (distractor valence: negative, neutral, positive) mixed-model ANOVA, with reward as the betweensubjects factor and the other factors within-subjects. Main effects of distractor valence $F(1.85,129.20)=11.01, p<.001, \eta_{p}^{2}=.136, \omega^{2}=.120$, and distractor type $F(1,70)=$ $122.36, p<.001, \eta_{p}{ }^{2}=.636, \omega^{2}=.596$, were qualified by a distractor type $\times$ distractor valence interaction $F(2,140)=30.94, p<.001, \eta_{p}{ }^{2}=.307, \omega^{2}=.281$, reflecting an emotional distraction effect. Comparing distraction indices [intact RT - scrambled RT] showed that the interaction was due to greater negative and positive than neutral distraction: negative distraction $(M=75 \mathrm{~ms}, S D=70 \mathrm{~ms})$ was greater than neutral distraction $(M=26 \mathrm{~ms}, S D=$ $22 \mathrm{~ms}), t(71)=6.73, p<.001, d_{z}=0.794,95 \% \mathrm{CI}[35,63]$; and positive distraction $(M=65$ $\mathrm{ms}, S D=64 \mathrm{~ms}$ ) was greater than neutral distraction, $t(71)=5.63, p<.001, d_{z}=0.663,95 \%$ 
Motivation and Emotional Distraction

CI $[25,53]$. Positive and negative distraction did not significantly differ, $t(71)=1.68, p=$ $.098, d_{z}=0.198,95 \%$ CI $[-2,22]$.

A main effect of reward confirmed that the reward group $(M=571 \mathrm{~ms}, S D=89 \mathrm{~ms})$ was faster than the control group $(M=654 \mathrm{~ms}, S D=126 \mathrm{~ms}), F(1,70)=10.40, p=.002, \eta_{p}{ }^{2}$ $=.130, \omega^{2}=.116$. The predicted reward group $\times$ distractor type interaction, $F(1,70)=10.14$, $p=.002, \eta_{p}^{2}=.127, \omega^{2}=.045$, was qualified by a reward group $\times$ distractor type $\times$ distractor valence interaction, $F(2,140)=5.20, p=.007, \eta_{p}^{2}=.069, \omega^{2}=.039$. Follow-up tests indicated that the three-way interaction was driven by a reduction in emotional, but not neutral, distraction with reward. Negative distraction was $42 \mathrm{~ms}$ lower in the reward group $(M=54 \mathrm{~ms}, S D=60 \mathrm{~ms})$ compared to the control group $(M=96 \mathrm{~ms}, S D=73 \mathrm{~ms}), t(67.8)=$ $2.70, p=.009, d_{s}=0.636,95 \% \mathrm{CI}[11,74]$; positive distraction was $46 \mathrm{~ms}$ lower in the reward group $(M=42, S D=48 \mathrm{~ms})$ compared to the control group $(M=88 \mathrm{~ms}, S D=70 \mathrm{~ms})$, $t(62.1)=3.24, p=.002, d_{s}=0.763,95 \%$ CI $[18,74]$. In contrast, for neutral distraction there was no significant difference between the reward $(M=22 \mathrm{~ms}, S D=18 \mathrm{~ms})$ and control $(M=$ $30 \mathrm{~ms}, S D=25 \mathrm{~ms}$ ) groups, $t(63.2)=1.46, p=.150, d_{s}=0.343,95 \% \mathrm{CI}[-3,18]$. No other effects reached significance.

To ensure that the overall speeding of RTs in the reward group did not account for the effect of reward on distraction, a mixed-model ANOVA with distractor valence (negative, neutral, positive) as the within-subjects factor and reward group (reward, control) as the between-subjects factor was conducted on proportional distraction indices [((intact RT scrambled RT) / scrambled RT)*100], which reflect slowing on intact distractor trials as a percentage of RTs on scrambled trials, thus controlling for this overall group difference in RT. Significant main effects of reward group, $F(1,70)=9.21, p=.003, \eta_{p}{ }^{2}=.116, \omega^{2}=.102$, and distractor valence, $F(2,140)=33.67, p<.001, \eta_{p}{ }^{2}=.325, \omega^{2}=.299$, were qualified by a significant reward group $\times$ distractor valence interaction $F(2,140)=4.92, p=.009, \eta_{p}{ }^{2}=$ $.066, \omega^{2}=.036$. Mirroring the RT findings, negative, $t(69)=2.57, p=.012, d_{s}=0.605,95 \%$ CI $[1,11]$, and positive, $t(64.2)=3.13, p=.003, d_{s}=0.736,95 \%$ CI $[2,11]$, but not neutral, $t(68.1)=0.90, p=.370, d_{s}=0.213,95 \% \mathrm{CI}[-1,2]$, proportional distraction indices were reduced by reward. These findings are consistent with the RT analyses, showing that the 
Motivation and Emotional Distraction

speeding of RTs in the reward group does not account for the benefit of reward on emotional distraction.

\section{Accuracy}

Accuracy (proportion correct) was high overall $(M=.917, S D=.054)$. As was done for the RT analysis, accuracy was analysed in a 2 (reward group: reward, control) $\times 2$ (distractor type: intact, scrambled) $\times 3$ (distractor valence: negative, neutral, positive) mixedmodel ANOVA. A main effect of reward showed that the reward group $(M=.899, S D=$ $.061)$ was less accurate than the control group $(M=.935, S D=.039), F(1,70)=8.82 p=$ $.004, \eta_{p}{ }^{2}=.112, \omega^{2}=.098$, indicating a speed-accuracy tradeoff. Despite this tradeoff, accuracy measures of distraction were consistent with the RT findings: Main effects of distractor valence, $F(2,140)=10.74, p<.001, \eta_{p}{ }^{2}=.133, \omega^{2}=.119$, and distractor type, $F(1$, $70)=43.17, p<.001, \eta_{p}{ }^{2}=.381, \omega^{2}=.366$, were qualified by a marginal distractor type $\times$ distractor valence interaction, $F(2,140)=2.51, p=.085, \eta_{p}{ }^{2}=.035, \omega^{2}=.020$. Follow-up tests revealed that negative distraction was marginally greater than neutral distraction, $t(71)=$ $1.79, p=.078, d_{z}=0.211,95 \%$ CI [-.002, .037]. Positive distraction was significantly greater than neutral distraction, $t(71)=2.17, p=.033, d_{z}=0.256,95 \%$ CI $[.002, .041]$. Positive and negative distraction did not differ from each other, $t(71)=0.387, p=.700, d_{z}=0.046,95 \%$ CI [-.025, .017]. RT and accuracy results converge to reflect greater emotional than neutral distraction. No other accuracy effects reached significance.

The main effect of reward group in both the RT and accuracy analyses indicates an overall speed-accuracy tradeoff; participants in the reward group were significantly ( $83 \mathrm{~ms})$ faster but also significantly less (3.6\%) accurate than those in the control group. Reward did not influence accuracy measures of distraction; the reward group $\times$ distractor type interaction was non-significant, $F(1,70)=1.16, p=.285, \eta_{p}{ }^{2}=.016, \omega^{2}=.001$. This suggests that a speed-accuracy tradeoff does not account for the effect of reward on RT distraction. To further ensure that the speed-accuracy tradeoff did not account for the effect of reward on distraction, we conducted a 2 (reward group: reward, control) $\times 2$ (distractor type: intact, scrambled) $\times 3$ (distractor valence: negative, neutral, positive) mixed-model ANOVA on inverse efficiency scores (adjusting RTs for accuracy: [RT/proportion correct]; see Bruyer \& Brysbaert, 2011). Consistent with the RT analysis, the reward group $\times$ distractor type interaction, $F(1,70)=6.05, p=.016, \eta_{p}^{2}=.080, \omega^{2}=.028$; and reward group $\times$ distractor type $\times$ distractor valence interaction, $F(2,140)=4.75, p=.010, \eta_{p}^{2}=.064, \omega^{2}=.038$, 
Motivation and Emotional Distraction

remained significant. Negative distraction, $t(63.4)=2.29, p=.025, d_{s}=.540,95 \%$ CI [9, $131]$, and positive distraction, $t(63.4)=2.62, p=.011, d_{s}=0.617,95 \% \mathrm{CI}[14,106]$, were both attenuated by reward, whereas neutral distraction did not differ between groups, $t(70)=$ $0.06, p=.951, d_{s}=0.014,95 \%$ CI $[-17,18]$. All other significant effects mirrored those in the RT analyses. These findings demonstrate that a strategy of sacrificing accuracy for speed does not account for the effect of reward on emotional distraction.

\section{Discussion}

We found that positive and negative emotional images were more distracting than neutral ones, but that emotional distraction was attenuated by the availability of performancecontingent reward. We had two hypotheses that led to opposing predictions. First, we reasoned that, because attentional biases to emotional stimuli are so strong, reward might have less influence on emotional than neutral distraction. Second, we reasoned that, because reward and emotional stimuli both have motivational value, reward might have a greater influence on emotional than neutral distraction. We were additionally interested in whether reward would influence positive and negative distraction to different degrees, because positive stimuli and reward both elicit approach motivation and positive emotion. We found that performance-contingent reward attenuated negative and positive, but not neutral, distraction. Thus, we found evidence for our second hypothesis; motivation influenced emotional to a greater extent than neutral distraction. Further, despite the different motivational functions of attending to positive and negative stimuli, we did not find evidence that reward influenced positive and negative distraction to different degrees. Current findings are consistent with those of Padmala and colleagues (Padmala \& Pessoa, 2014; Padmala et al., 2017) who showed a benefit of reward for controlling negative distraction (relative to a neutral image baseline). We extend these findings to show that motivation enhances control of negative and positive distractions, under conditions that elicited a large emotional distraction effect in the control condition.

Our findings indicate that the level of distraction is dependent on distractor arousal, rather than valence; high arousal distractors (both positive and negative) impaired performance to a greater extent than low arousal (neutral) distractors. Moreover, the fact that reward attenuated positive and negative, but not neutral, distraction suggests that the impact of motivation on control is also dependent on distractor arousal, rather than distractor valence. The benefit of reward for both negative and positive distraction parallels the effect 
Motivation and Emotional Distraction

of increased distractor frequency in a similar task (Grimshaw et al., 2018). According to the DMC framework (Braver et al., 2007), both expectations (manipulated by distractor frequency), and motivation (manipulated by reward), act in a top-down manner to shift control from a reactive strategy (triggered when distraction occurs) to a proactive one (using anticipatory control to prevent distraction before it occurs). The influence of reward on proactive control has been repeatedly demonstrated in emotionally-neutral contexts (see Botvinick \& Braver, 2015). Here, we show that it might be possible to extend this model to contexts in which distractors are negative and positive images.

However, it is also possible that motivation enhances reactive control processes to reduce emotional distraction, rather than eliciting a shift to proactive control. Because attentional biases to emotion are important for survival (LeDoux, 2012; Rolls 2000), perhaps we always attend to emotional content before determining whether it needs further attention and action. In this case, we would use reactive control processes to shift attention back to our task. The question of how motivation influences the timing of implementation of control mechanisms to reduce emotional distraction is addressed in Chapter 3.

Unlike emotional distraction, neutral distraction was not significantly reduced by reward. Neutral distraction was relatively low in the control group, suggesting a possible floor effect. Or, the effect of reward could be too small to be detected in this study; numerically, neutral distraction was lower in the reward group than in the control group, but the effect was not statistically significant. Alternatively, as outlined in the introduction to this chapter, the reward group $\times$ distractor type $\times$ distractor valence interaction could reflect a difference in the mechanisms by which reward influences control of emotional versus neutral distraction. Reward might enhance the motivational value of the letter targets, which would influence goal-driven attentional competition between the letters and emotional distractors to a greater degree than neutral ones. I return to this point in Chapter 5: General Discussion.

Our finding that reward reduces both negative and positive distraction contrasts with reports of other attentional manipulations that attenuate negative but not positive distraction. Notably, those studies manipulated perceptual load (Gupta et al., 2016) and attentional settings (Most et al., 2007) to affect control. These discrepancies logically suggest that multiple control mechanisms may be used to control emotional distractions, depending on the factor that elicits control (e.g., perceptual load vs. reward). Further studies to directly compare the effects of different manipulations will be useful for identifying the conditions under which different control mechanisms are engaged, and their influence on positive versus negative distraction, and on emotional versus neutral distraction. Online measures of control 
Motivation and Emotional Distraction

(e.g., pupillometry or electrophysiology; e.g., Chiew \& Braver, 2013, 2014) will provide insight into the timecourse of control elicited by different manipulations. The next chapter describes an experiment in which we replicate the task from Experiment 1, while measuring pupil size to index of the timecourse of cognitive effort (van der Wel \& van Steenbergen, 2018). The goal of Experiment 2 is to examine how motivation influences the timecourse of cognitive control of emotional distraction, to test predictions from the DMC framework regarding whether reward reduces emotional distraction by eliciting a shift to proactive control, as it does in emotionally-neutral contexts. Alternatively, reward might reduce emotional distraction by upregulating reactive control processes after emotional distractors have been selected for attention. 
Motivation and Emotional Distraction

Chapter 3. Reward elicits cognitive control over emotional distraction: Evidence from pupillometry.

Portions of this chapter appear in: Walsh, A. T., Carmel, D., \& Grimshaw, G. M. (2018). Reward elicits cognitive control over emotional distraction: Evidence from pupillometry. Cognitive, Affective, \& Behavioural Neuroscience. https://doi.org/10.3758/s13415-01800669-w

Link to preprint: https://psyarxiv.com/k4z6u/

Open data, materials, code, and analyses for published manuscript: https://osf.io/yhkdr/ Link to preregistration: https://osf.io/jd96p/ (also see Appendix C).

Open data and code to reproduce pupil analyses for this chapter are available at https://osf.io/yhkdr/ (they are the same as in the published manuscript).

Open data and code to reproduce behavioural analyses for this chapter are available at https://osf.io/vxwd4/

Experiment 1 showed that motivation, elicited by performance-contingent reward, reduced distraction from potent negative and positive emotional distractors. A central tenet of the DMC framework is that, in non-emotional contexts, motivation induces a shift from a reactive control strategy to a more effective proactive control strategy, resulting in less distraction (see Chapter 1: General Introduction for a review). Evidence for this shift to proactive control comes from the influence of reward on patterns of behaviour, and pupil dilation, in the AX-CPT (e.g., Chiew \& Braver, 2013, 2014), and neuroimaging studies (e.g., Padmala \& Pessoa, 2011). This proactive shift is thought to be implemented via connections between reward-processing areas (such as the ventral striatum and orbitofrontal cortex) and frontoparietal regions (such as the anterior cingulate cortex and dorsolateral prefrontal cortex; Botvinick \& Braver, 2015; Pessoa \& Engelmann, 2010; Pessoa, 2009) that influence visual processing areas, facilitating attentional control. However, it is not yet known whether motivation reduces emotional distraction via a similar shift to proactive control.

It is reasonable to think that reward might enhance control of emotional distractions via different mechanisms than it does for neutral distractions. Emotional distractors (unlike their neutral counterparts) are thought to bias our attention via exogenous, endogenous, and selection history processes (Awh et al., 2012; Brosch et al., 2011; Mohanty \& Sussman, 2013; Mulckhuyse, 2018; Sussman et al., 2016), making them more difficult to ignore (OkonSinger et al., 2007; Pourtois et al., 2013; Yiend, 2010). Emotional stimuli may even capture attention via emotion-specific mechanisms (Pourtois et al., 2013; Vuilleumier, 2015). Attention is heavily biased toward biologically-relevant emotional stimuli (Yiend, 2010) because they are important for survival and reproduction (LeDoux, 2012; Rolls, 2000). 


\section{Motivation and Emotional Distraction}

Therefore, it might be adaptive for them to always capture our attention, regardless of their relevance to current goals, so that we can determine whether they require immediate action before we disengage from them. If emotional distractors bias our attention reflexively, we may not be able to control them proactively. Instead, we may be more likely to control emotional distraction by upregulating reactive control so that we can rapidly disengage from distractors. Although behavioural findings from Experiment 1 are consistent with a shift to proactive control, it is also possible that motivation facilitated reactive control processes to reduce emotional distraction. Our primary research question is therefore whether the availability of rewards encourages proactive control when potential distractors are emotional, just as it does in emotionally-neutral contexts (Botvinick \& Braver, 2015; Yee \& Braver, 2018).

Recent neuroimaging studies provide more direct support for the proactive control of emotional distraction under conditions of reward. In two studies, Padmala and colleagues (Padmala \& Pessoa, 2014; Padmala et al., 2017) had participants ignore a centrally-presented distractor image while judging the orientation of two flanking lines. Participants were slower when negative compared to neutral distractors were presented (i.e., they showed an emotional distraction effect). On some trials, a pre-trial reward cue appeared, indicating availability of monetary reward for fast and accurate performance on the upcoming trial. Reward sped up responses, and also eliminated emotional distraction. Importantly, fMRI showed that participants with greater ventral striatum activation during the reward cue phase showed a greater benefit from reward on a behavioural measure of emotional distraction (Padmala et al., 2017). Furthermore, there was stronger functional connectivity between ventral striatum and frontal and parietal regions involved in attentional control while processing reward compared to non-reward cues, consistent with the recruitment of proactive control mechanism to reduce emotional distraction when rewards were signalled.

Although neuroimaging findings are consistent with the hypothesis that reward encourages the implementation of proactive control, dynamic changes in control are best examined using more temporally-sensitive measures of cognitive and emotional processing. The current preregistered study uses pupillometry (a high-resolution measure of changes in pupil area over time), to examine the effect of reward on the timecourse of cognitive control of emotional and neutral distractions. Pupil dilation marks changes not only in perceived luminance (Mathôt \& Van der Stigchel, 2015), but also in cognitive processing; dilation is an established, temporally-sensitive, measure of cognitive control (Beatty \& Lucero-Wagoner, 2000; Chatham et al., 2009; Chevalier et al., 2015; Chiew \& Braver, 2013, 2014; Jones, 


\section{Motivation and Emotional Distraction}

Siegle, \& Mandell, 2015; Kahneman, 1973; Rondeel, van Steenbergen, Holland, \& van Knippenberg, 2015; Rondeel et al., 2015). Pupil dilation is driven by release of the neuromodulator norepinephrine (NE) from the locus coeruleus (LC; Joshi, Li, Kalwani, \& Gold, 2016; Laeng, Sirois, \& Gredebäck, 2012; Rajkowski, Majczynski, Clayton, \& AstonJones, 2004). The LC (located in the rostral pons) provides the major source of NE to the brain, sending dense projections to diverse brain regions, including those involved in attentional control (e.g., PFC, parietal cortex, superior colliculus, and the pulvinar nucleus) and reward processing (including the ventral tegmental area, eliciting dopaminergic release from the nucleus accumbens; see Laeng et al., 2012; Sara, 2009 for reviews). It is thought that NE has an important arousal-inducing function, which promotes cognitive performance (Laeng et al., 2012; Samuels \& Szabadi, 2008; Sara, 2009). Furthermore, NE is thought to facilitate changes to attention, cognition and behaviour by enhancing functional connectivity between various brain regions (Coull, Büchel, Friston, \& Frith, 1999; Sara, 2009).

Pupillometry is sensitive to both emotional arousal (e.g., Bradley, Miccoli, Escrig, \& Lang, 2008; Henderson, Bradley, \& Lang, 2014) and cognitive effort (Kahneman, 1973; Laeng et al., 2012; van der Wel \& van Steenbergen, 2018), and so is well-suited to our aims.

Proactive control refers to a constellation of control mechanisms that are implemented in anticipation of distraction. Proactive control mechanisms can be implemented in a sustained, stable manner over an extended period of time (e.g., sustained activation of the goal representation in working memory across a block of trials); or dynamically (e.g., suppressing visual processing in a predicted distractor location just before distractor onset). Two measures of pupil dilation are used to capture sustained and dynamic changes in proactive control respectively: tonic dilation indexes sustained proactive control, and phasic dilation indexes dynamic proactive control. Tonic dilation refers to changes in baseline pupil area across a block of trials (measured during an inter-trial-interval period; e.g., Chiew \& Braver, 2013, 2014; Heitz, Schrock, Payne, \& Engle, 2008), and reflects state-related changes in arousal that accompany sustained activation of proactive control mechanisms. Tonic dilation is subtraction baselined to tonic dilation in the inter-trial-interval during the baseline block (in which no reward is available). In contrast, phasic dilation is an event-related transient change triggered by a specific stimulus or event (baselined to an immediately preceding time-period). Pre-stimulus phasic dilation reflects transient increases in effort that accompany dynamic activation of proactive control mechanisms. Recently, Chiew and Braver $(2013,2014)$ used both tonic and phasic pupil dilation to measure the timecourse of motivationally-induced enhancements of cognitive control in an emotionally-neutral context. 


\section{Motivation and Emotional Distraction}

Participants were rewarded for successful performance on a response conflict task, the AXCPT, which elicited behavioural performance consistent with a shift toward proactive control. Reward increased tonic pupil dilation, thought to reflect increases in effort that accompany a shift to a sustained proactive control strategy. Reward also increased preparatory phasic pupil dilation (triggered by the cue for an impending target), thought to reflect a more dynamic form of proactive control (Chatham et al., 2009; Chevalier et al., 2015). These findings suggest that both tonic and phasic changes in pupil dilation can mark motivationally-driven shifts to proactive control, at least in an emotionally-neutral context.

Pupil dilation also indexes emotional arousal induced by viewing emotional images (Bradley et al., 2008; Cohen et al., 2015; Henderson et al., 2014; Kinner et al., 2017; Snowden et al., 2016; Vanderhasselt, Remue, Ng, \& De Raedt, 2014), and correlates with measures of skin conductance (a measure of sympathetic nervous system arousal; Bradley et al., 2008). This emotion-driven pupil response is subject to top-down influences. When cognitive control is enhanced via conflict manipulations that elicit conflict adaptation (incongruent trials on a flanker task; Cohen, et al., 2015), or by implementation of effective emotion-regulation strategies (Kinner et al., 2017; Vanderhasselt et al., 2014), pupil dilation in response to negative emotional images is reduced. Furthermore, pupil dilation during anticipation of a negative emotional image is inversely related to pupil dilation in response to the image itself (Vanderhasselt et al., 2014). Therefore, increased anticipatory pupil dilation can index proactive control of emotional processing, and reduced post-stimulus pupil dilation can index the subsequent attenuated processing of emotional content.

The current study takes advantage of the pupil's sensitivity to the timing of cognitive effort and emotional processing, to determine whether reward reduces emotional distraction via a shift to proactive control. The behavioural task replicated Experiment 1 . We therefore expected that positive and negative images would be more distracting than neutral images (a distractor type $\times$ distractor valence interaction). We predicted that reward would speed up response time and would reduce distraction overall (a reward group $\times$ distractor type interaction). Furthermore, we expected that the motivational manipulation would again enhance control of emotional distraction to a greater extent than neutral distraction, reflected by reduced behavioural emotional distraction under reward (i.e., reward group $\times$ distractor type $\times$ distractor valence interaction). The key question was whether reward improves performance by encouraging people to shift to a proactive control strategy to ignore emotional distractors. If reward elicits proactive control via a sustained strategy that is maintained across an experimental block, we expected reward to increase tonic pupil dilation 


\section{Motivation and Emotional Distraction}

in the inter-trial-interval. If reward elicits proactive control via a dynamic strategy that is implemented on a trial-by-trial basis, we expected reward to enhance phasic, pre-stimulus pupil dilation. Additionally, we examined phasic pupil dilation in the post-stimulus period, to provide insight into the consequences of control for subsequent distractor processing. Specifically, we predicted that reward would reduce pupil dilation to intact (relative to scrambled) distractors, reflecting reduced distractor processing. Lastly, we examined the reward group $\times$ distractor valence interactions in all planned pupil analyses, to determine whether reward differently influences the timecourse of proactive control (indexed by pupil dilation) of neutral, negative and positive distractions.

\section{Method}

This study was preregistered on Open Science Framework (OSF) prior to data collection (link: https://osf.io/jd96p/; see Appendix C). The preregistration adheres to the disclosure requirements of OSF. Data and code for behavioural analyses for this chapter can be found at https://osf.io/vxwd4/. Data and code for pupil analyses are the same here as in the published manuscript which are stored on the OSF page for the published manuscript https://osf.io/yhkdr. Other materials (e.g., code for image manipulation) from the published manuscript and this chapter are available at https://osf.io/yhkdr.

\section{Participants and Sample Size Determination}

To determine the sample size needed to test the effect of reward on the pupil's response to emotional images, we assumed that the effect of reward on post-stimulus pupil dilation would be comparable in size to the effect of reward on behavioural emotional distraction (RT), which was $d_{s}=0.519$ in Experiment 1 . This effect size is similar to that reported by Cohen et al. $\left(2015 ; d_{s}=0.503\right)$ for the effect of flanker congruency on pupillary response to negative (relative to neutral) images. A sample size analysis in G*Power (Faul et al., 2007) indicated 47 participants per group would provide sufficient power to detect a similar effect of reward on emotional distraction $80 \%$ of the time (alpha $=.05$ ). Rounding up to accommodate counterbalancing yielded a sample size of 54 participants per reward group. A sensitivity analysis (also in $G^{*}$ Power, with alpha $=.05$ ) showed that 54 participants per group results in an $80 \%$ probability to detect an effect of $d_{s}=0.482$ or larger (alpha $=.05$ ).

A total of 114 participants were recruited from the community in Wellington, New Zealand, via fliers, emails, and social media posts. Participants were randomly allocated to the control or reward groups. Four participants did not complete the task due to technical errors (experiment crashing $n=3$, eye-tracker technical issues $n=1$ ). Replacement participants were run to reach the sample size goal, resulting in 110 participants in the final 
Motivation and Emotional Distraction

sample (31 men, 79 women; mean age 23 years; range 18-39 years) who were randomly split into the reward and control groups ( $n=55$ per group). We exceeded the target of 54 participants per condition because a participant who was mistakenly excluded and replaced did not actually meet the preregistered exclusion criteria; an additional participant was therefore recruited to balance the size of the control and reward groups. No participants met the preregistered exclusion criteria (see Data exclusion criteria, below).

\section{Materials}

The experiment was run on an Acer PC with a 22" flat-screen monitor with $1024 \times$ 768 pixel resolution and a $120 \mathrm{~Hz}$ refresh rate. Viewing distance was maintained by a chinrest $60 \mathrm{~cm}$ from the screen. The experiment was programmed and run on E Prime 2.0 (Psychology Software Tools, Pittsburgh, PA). A nine-point calibration and validation of the eye-tracker was performed at the beginning of the session. Between each block a manual drift check was performed, and if necessary, the calibration and validation were performed again. Area of the left pupil (in arbitrary units) was recorded using an Eyelink 1000-plus desktop mounted eye-tracker (SR Research Ltd., Mississauga, ON) using a $1000 \mathrm{~Hz}$ sampling rate.

The image sets were the same as in Experiment 1, but instead of pixel scrambles, phase scrambled images were created (see an example in Figure 3.1). Pixel scrambles (as were used in Experiment 1) create many abrupt changes to contrast and luminance on a local level; whereas phase scrambles create smoother changes to contrast and luminance. Given that the pupil dilates in response to changes in luminance and contrast, we reasoned that phase scrambles may result in a less noisy pupil signal than pixel scrambles. Three scrambled versions of each image were used as control stimuli so that each image (including intact and scrambled images) would be presented the same number of times across the experiment. The scrambled images were created in MATLAB by performing a 2D fast Fourier transform, followed by phase randomisation, and reconstruction of the image. The process of phase scrambling also matched all images on average root mean square contrast. The scrambled images maintain the same lower level visual properties (i.e. colour, amplitude spectrum) as the original image while changing the position of the spatial frequency components and removing meaningful content. All images (intact and scrambled) were then matched on average luminance using the SHINE MATLAB toolbox (Willenbockel et al., 2010).

\section{Task and procedure}

Figure 3.1 depicts the distraction task. 


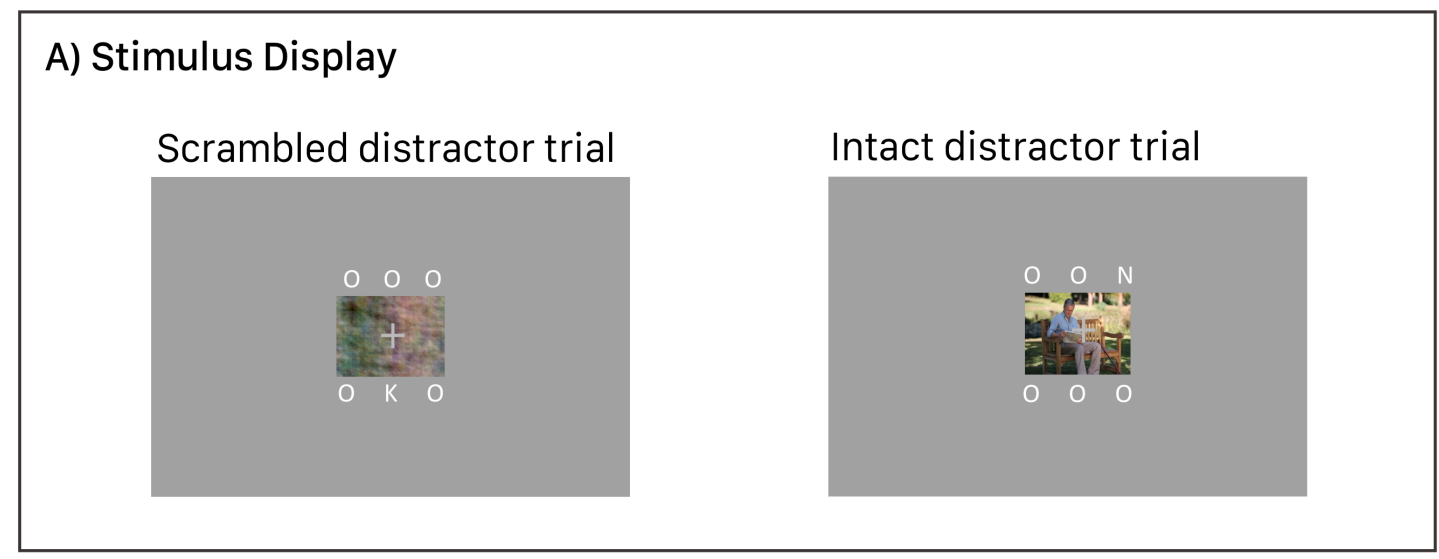

B) Trial Sequence

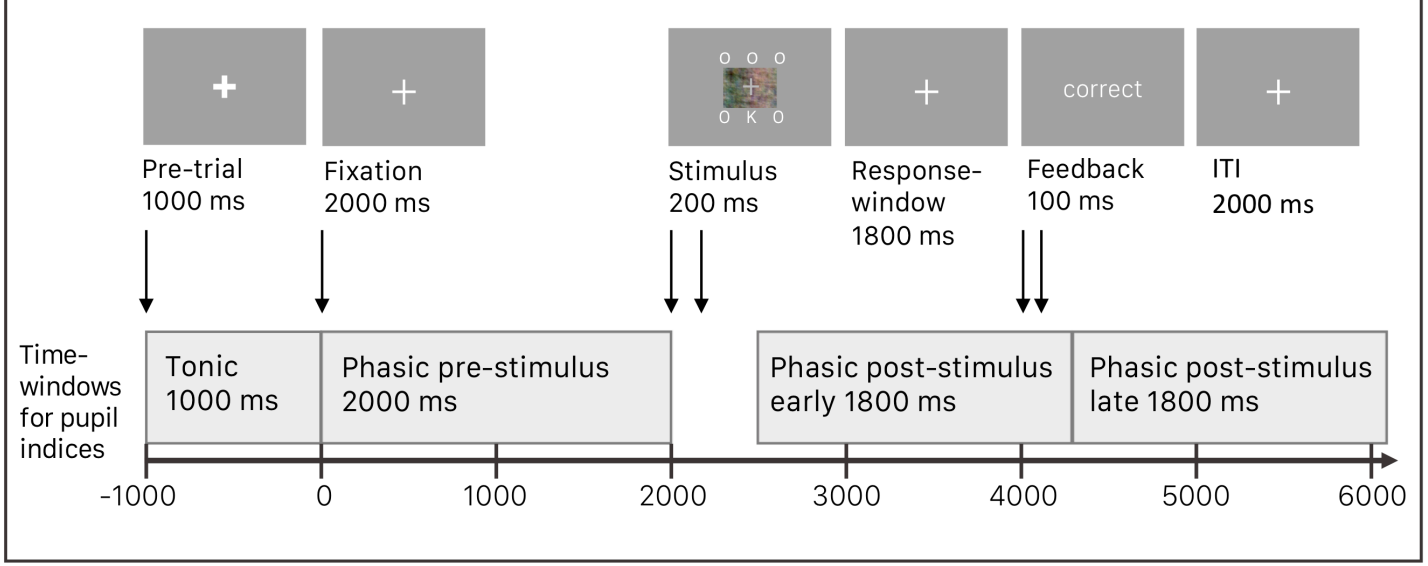

Figure 3.1. Target display (A) and trial sequence (B) in Experiment 2. A) An example of a scrambled (left) and intact (right) target display. B) The trial sequence. Arrows indicate onset of a display. Preregistered time-windows for pupil analyses are represented by grey shaded boxes. For illustrative purposes, images are not to scale; the central part of the display is enlarged.

Participants were tested in a dimly-lit room. Participants were told that the images were irrelevant to their task, so they should ignore them. They were asked to avoid blinking excessively, to keep their head still, and to continue looking at the centre of the screen throughout a trial.

For all participants (in both reward and control conditions), each trial began with a large, bold white fixation cross on a grey background. When they were ready to start the trial, the participant looked directly at the bold cross for $1000 \mathrm{~ms}$, at which point it turned into a smaller white fixation cross indicating the start of the trial. The smaller fixation cross was presented for a constant duration of $2000 \mathrm{~ms}$ and was followed by the target display for 200 ms. The target display was the same as in Experiment 1 but was presented on a grey 


\section{Motivation and Emotional Distraction}

background. In the centre of the image was a light grey cross which provided a focal point for participants to help them control eye-movements so we could accurately measure pupil size. The image was an intact IAPS image on $25 \%$ of trials, and a scrambled image on $75 \%$ of trials. As in Experiment 1, participants indicated (as quickly and accurately as possible) whether the target letter was a $\mathrm{K}$ or an $\mathrm{N}$, using the keys 1 and 2 on the number pad, with the index and middle fingers of their right hand. Following the target display, the fixation cross reappeared for $1800 \mathrm{~ms}$ (a fixed response-window, regardless of when a response was made). Feedback was then displayed for $100 \mathrm{~ms}$, indicating whether the participant was "correct" or "incorrect" in white font. If no response was made within the $1800 \mathrm{~ms}$ response-window then the feedback stated: "please respond faster". Following the feedback, the smaller fixation cross appeared again throughout the $2000 \mathrm{~ms}$ inter-trial-interval. At the end of a trial, the larger bold fixation cross again appeared, and the next trial began when the participant had fixated on it for $1000 \mathrm{~ms}$.

The experiment began with a set of 12 practice trials, arranged in three mini-blocks (negative, neutral, positive) of four trials each, with one intact and three scrambled images appearing randomly in each mini-block. The images presented in the practice trials were different from those used in experimental blocks. Following the practice trials was a baseline block of 24 trials. As in Experiment 1, the purpose of the baseline block was to calculate participants' individual median RT, to be used as a threshold for reward during the experimental blocks and to provide a baseline from which to measure tonic pupil dilation in subsequent blocks. The baseline block consisted of 24 scrambled distractor trials, with no intact distractor trials. This differs from Experiment 1 - in which there were 12 intact neutral distractor trials in the baseline block. Because the baseline block trials were being used as a baseline for calculating tonic pupil indices, I did not want this baseline to be influenced by distractor meaning. The phase scrambled images used in the baseline block were derived from a set of 12 neutral images (different from those used in the neutral experimental blocks) and were presented once each. Following the baseline block, participants completed six experimental blocks of 48 trials each. Each block comprised 12 different intact and 36 scrambled image trials (in random order), all with the same valence. There were two sequential blocks of each valence (e.g. positive, positive, negative, negative, neutral, neutral), resulting in each intact and scrambled image being presented twice in total. Valence order was counterbalanced across participants.

As in Experiment 1, the Behavioural Activation and Inhibition Scale (Carver \& White, 1994) was administered to participants at the end of the experimental session for 


\section{Motivation and Emotional Distraction}

exploratory analyses, however due to a technical error the responses were not recorded and were therefore not analysed.

Reward Manipulation. Following the baseline block, the reward group was informed of the potential to earn points and move up levels in the following six blocks of trials. They were told that winning points would lead to achieving levels, and that each new level increased total earnings upon task completion by $\$ 2$. Participants began on level one (total earnings: \$10). Level two required 53 points (total earnings: \$12); 53 additional points were needed to ascend to each new level, up to level 6 (total earnings: \$20). Note that this differs slightly from the payoff scheme in Experiment 1, partly due to the reduced number of trials in the current experiment (because of the $\mathrm{ABC}$ valence block structure here, in contrast to the ABBA valence block structure in Experiment 1), and to also increase the perception of progress for the participant. However, all participants (including those in the control group) actually earned \$20 in supermarket vouchers at the end of the study, regardless of the amount they presumed they had won during the study. Visual feedback was provided after every trial: if participants responded accurately and faster than their median response time in the baseline block, they earned 1 point, and saw: "you won a point!"; if they were accurate but slower than the reward threshold they saw "correct", and if they were incorrect saw "incorrect". Feedback on overall performance was provided in each break between blocks. If a participant moved up a level in the preceding block, the feedback screen read, "Well done! You have moved up a level." If not, it read "No level up". On the same screen, they saw their current point total, current level, and mean proportion correct for the previous block. Participants were reminded at this point to be fast and accurate. To minimise the potential for a speedaccuracy tradeoff due to the reward RT deadline, a warning to keep trying to be accurate was provided during the breaks between blocks if accuracy fell below 95\% across the previous block.

The control group performed the task with no performance-contingent reward. They received accuracy feedback after each trial ("correct", "incorrect", or "please respond faster"). They did not have the opportunity to earn points and did not receive overall performance feedback between blocks. Instead they saw a screen saying, "please take a short break".

\section{Preregistered analyses}

The following descriptions of data processing, calculation of indices, inference and exclusion criteria, and analyses were all preregistered. 
Motivation and Emotional Distraction

Pupillometry data processing. Pupillometry data were pre-processed in MATLAB using a combination of adapted open source code (retrieved from http://www.smanohar.com/matlib.php), and our own code. Blinks $<500 \mathrm{~ms}$ long were linearly interpolated. Partial blinks were identified by extreme (unrealistic) changes in pupil area from one time point to the next; pupil area during these partial blinks was then linearly interpolated. Pupil indices were calculated using mean pupil area (in arbitrary units) across several time-windows (as outlined in more detail below). Baseline subtraction was used to calculate indices, as it is more robust to baseline artifacts, and increases statistical power, relative to divisive baseline corrections (Mathôt, Fabius, VanHeusden, \& Stigchel, 2017). Only trials with correct responses were included in phasic pupil indices.

The first two time-windows (tonic and phasic pre-stimulus; see Figure 3.1B) provide indices to test predictions regarding the effects of reward on anticipatory, proactive control. The first window provides an index of tonic proactive control: mean pupil area across the $1000 \mathrm{~ms}$ time-window prior to trial-onset, baselined to the grand mean of pupil area across the $200 \mathrm{~ms}$ time-window immediately preceding trial-onset across all 24 (non-reward, scrambled image only) baseline block trials. The second window provides an index of phasic proactive control: mean pupil area across the $2000 \mathrm{~ms}$ time-window starting from fixationonset, and ending at stimulus-onset, baselined to mean pupil area across the $200 \mathrm{~ms}$ timewindow immediately preceding fixation-onset. This phasic proactive control index reflects dynamic, effortful preparation immediately preceding stimulus-onset.

Two phasic post-stimulus (early and late) indices were created to test predictions regarding how reward affects the pupil's response to the emotional distractors. The early post-stimulus window begins $500 \mathrm{~ms}$ post-stimulus-onset and extends for $1800 \mathrm{~ms}^{4}$. The late post-stimulus window begins immediately following the first and extends for $1800 \mathrm{~ms}$ until the end of the trial. For both the early and late post-stimulus indices, the baseline is the mean pupil area $200 \mathrm{~ms}$ immediately preceding stimulus-onset. These post-stimulus phasic indices for intact image trials are subtracted from the phasic indices on scrambled image trials (thus controlling for pupil dilation in response to low-level visual properties) to obtain an index of the pupil's response to the content of the intact images. All indices were calculated for each individual participant, separately for each valence.

\footnotetext{
${ }^{4}$ Other (unpublished) studies from our lab, in which participants passively viewed these same images, typically find that effects of distractor type (intact versus scrambled) and valence on pupil dilation did not begin to emerge until $500 \mathrm{~ms}$ post-stimulus-onset.
} 
Motivation and Emotional Distraction

Data exclusion criteria. Trials were excluded if they were missing more than $50 \%$ of pupil samples. Additionally, trials were excluded from specific analyses if the baseline period for that time-window was missing more than $50 \%$ of its pupil area samples. A mean of 13 (out of 288) indices were removed per participant $(\min =0, \max =66)$. Our preregistration stated that participants would be excluded if they had less than $75 \%$ accuracy overall, less than $60 \%$ accuracy on a given block, loss of more than $20 \%$ of their pupil samples overall, or had fewer than 10 trials in a given condition. No participants met these exclusion criteria.

Inference criteria. $P$ values $<.05$ were accepted as statistically significant and relevant follow-up analyses were conducted on these effects. One- or two-tailed tests for planned comparisons were specified in the preregistered predictions, and were not corrected for multiple comparisons (Armstrong, 2014). Exploratory analyses were not corrected for multiple comparisons (except for the pupil - behaviour correlations), but are noted as exploratory and should be interpreted with caution. Note that distractor type (intact, scrambled) is not a factor in the analyses because it is either irrelevant (for the proactive indices), or included in the transformations (for the post-stimulus indices).

\section{Results}

All analyses were preregistered unless noted as exploratory. All preregistered predictions are reported as supported, or not supported. Significant findings that were not predicted are noted as such. Data and code to reproduce analyses and figures from the published paper are provided on OSF (https://osf.io/yhkdr/). Data and code to reproduce analyses and figures for this thesis are provided on OSF (https://osf.io/vxwd4/). For consistency with the other experiments in this thesis, the following small changes to the preregistered analysis plan were made, that also differ from the content of the published paper: follow-up tests that were preregistered as one-tailed are presented here as two-tailed; valence effects that were preregistered as quadratic tests (with neutral entered as the middle level) are presented here as non-quadratic; and positive and negative behavioural distraction were separately analysed in follow-up tests, rather than collapsing into emotional distraction. These deviations all result in a more conservative statistical approach than was taken in the publication, and in the preregistration, and did not influence the statistical significance of any effects or interactions.

\section{Response Times}

Mean correct RTs and accuracy for each condition, and comparisons between intact and scrambled conditions, are presented in Table 3.1. See Figure 3.2 for mean distraction [intact RT - distractor RT] in each condition. 
Motivation and Emotional Distraction

Table 3.1. Comparisons of mean correct RTs (ms) and accuracy (proportion correct) for scrambled versus intact images (distraction indices in $\mathrm{ms}$ ), for each distractor valence condition, separately for control and reward groups for Experiment 2. SDs are in brackets.

\begin{tabular}{llllcc}
\hline Valence & Scrambled & Intact & Distraction & $d_{z}$ & \multicolumn{2}{c}{ 95\% CI } \\
Block & Trials & Trials & & & \\
\hline
\end{tabular}

$\underline{\text { Response Times }}$

Control Group

\begin{tabular}{lllllll} 
Negative & $669(91)$ & $813(152)$ & $144 * * *(93)$ & 1.550 & 119 & 169 \\
Neutral & $670(81)$ & $726(112)$ & $56 * * *(49)$ & 1.145 & 43 & 69 \\
Positive & $674(94)$ & $825(160)$ & $151 * * *(93)$ & 1.636 & 126 & 176 \\
\multicolumn{2}{l}{ Reward Group } & & & & & \\
Negative & $562(100)$ & $620(140)$ & $58^{* * *(60)}$ & 0.978 & 42 & 75 \\
Neutral & $564(86)$ & $598(117)$ & $34 * * *(42)$ & 0.824 & 23 & 46 \\
Positive & $568(111)$ & $631(145)$ & $63 * * *(52)$ & 1.204 & 49 & 77
\end{tabular}

\section{$\underline{\text { Proportion Correct }}$}

\section{Control Group}

\begin{tabular}{lllllll} 
Negative & $.965(.036)$ & $.947(.052)$ & $.018^{*}(.055)$ & 0.327 & .003 & .033 \\
Neutral & $.973(.030)$ & $.966(.046)$ & $.007(.044)$ & 0.156 & -.005 & .019 \\
Positive & $.969(.025)$ & $.926(.070)$ & $.043 * * *(.070)$ & 0.631 & .025 & .063 \\
Reward Group & & & & & & \\
Negative & $.955(.037)$ & $.915(.081)$ & $.040 * *(.072)$ & 0.560 & .021 & .059 \\
Neutral & $.960(.042)$ & $.946(.054)$ & $.014 *(.045)$ & 0.314 & .002 & .026 \\
Positive & $.952(.038)$ & $.909(.083)$ & $.043 * * *(.075)$ & 0.567 & .063 & .022 \\
\hline
\end{tabular}

Note: Distraction is calculated as: [RT intact - RT scrambled] and [proportion-correct scrambledproportion-correct intact]. Effect sizes are Cohen's $d_{z}$ from paired comparisons of intact and scrambled trials within conditions, and asterisks indicate whether the [intact-scrambled] difference is significant in that condition. ${ }^{*} p<.05, * * * p<.001 .95 \%$ confidence intervals surround the distraction scores, in ms and proportion correct. $n=55$ per group. 
Motivation and Emotional Distraction

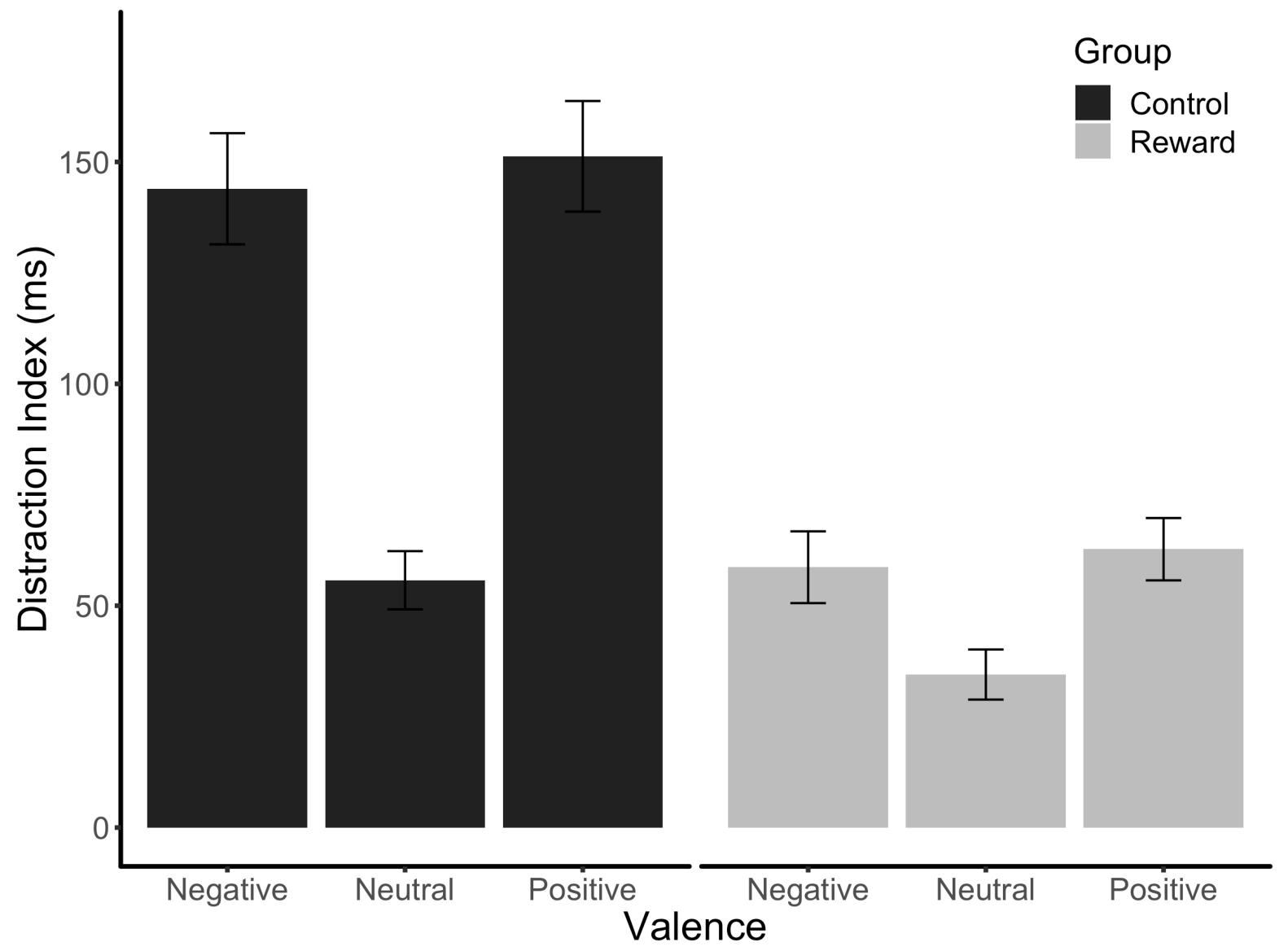

Figure 3.2. Mean behavioural distraction indices (in ms) by reward group and valence for Experiment 2. Greater distraction [RT intact - $R T$ scrambled] was elicited by emotional than by neutral images. Neutral and (to a greater extent) emotional distraction were attenuated by reward. Error bars represent standard error of the mean.

To test hypotheses regarding the influence of reward on control of emotional and neutral behavioural distraction, correct RTs were analysed in a 2 (reward group: reward, control $) \times 2$ (distractor type: intact, scrambled) $\times 3$ (distractor valence: negative, neutral, positive) mixed-model ANOVA, with reward as the between-subjects factor and the other factors within-subjects. Main effects of distractor valence $F(2,216)=19.91, p<.001, \eta_{p}{ }^{2}=$ $.156, \omega^{2}=.141$, and distractor type $F(1,108)=276.48, p<.001, \eta_{p}{ }^{2}=.719, \omega^{2}=.646$, were qualified by the predicted distractor type $\times$ distractor valence interaction $F(2,216)=48.67, p$ $<.001, \eta_{p}{ }^{2}=.311, \omega^{2}=.277$. Comparing distraction across conditions showed that the interaction was due to greater negative and positive than neutral distraction: negative distraction $(M=101 \mathrm{~ms}, S D=89 \mathrm{~ms})$ was greater than neutral distraction $(M=45 \mathrm{~ms}, S D=$ $46 \mathrm{~ms}), t(109)=7.701, p<.001, d_{z}=0.734,95 \% \mathrm{CI}[42,71]$; and positive distraction $(M=$ $107 \mathrm{~ms}, S D=87 \mathrm{~ms}$ ) was greater than neutral distraction, $t(109)=7.85, p<.001, d_{z}=0.748$, 
Motivation and Emotional Distraction

$95 \%$ CI $[46,78]$. Positive and negative distraction did not significantly differ, $t(109)=0.83, p$ $=.410, d_{z}=0.079,95 \%$ CI $[-19,8]$.

The predicted main effect of reward confirmed that the reward group $(M=590 \mathrm{~ms}$, $S D=112 \mathrm{~ms})$ was faster than the control group $(M=729 \mathrm{~ms}, S D=104 \mathrm{~ms}), F(1,108)=$ $45.50, p<.001, \eta_{p}{ }^{2}=.296, \omega^{2}=.288$. The predicted reward group $\times$ distractor type interaction, $F(1,108)=40.95, p<.001, \eta_{p}{ }^{2}=.275, \omega^{2}=.094$, was qualified by the significant reward group $\times$ distractor type $\times$ distractor valence interaction, $F(2,216)=14.97, p<.001$, $\eta_{p}{ }^{2}=.122, \omega^{2}=.081$. Follow-up tests showed that this three-way interaction was driven by a greater reduction in emotional compared to neutral distraction with reward: negative distraction was $102 \mathrm{~ms}$ lower in the reward group $(M=59 \mathrm{~ms}, S D=60 \mathrm{~ms})$ compared to the control group $(M=144 \mathrm{~ms}, S D=93 \mathrm{~ms}), t(92.4)=5.72, p<.001, d_{s}=1.09,95 \%$ CI [56, 115]. Positive distraction was $88 \mathrm{~ms}$ lower in the reward group $(M=63 \mathrm{~ms}, S D=52 \mathrm{~ms})$ compared to the control group $(M=151, S D=92 \mathrm{~ms}), t(85.2)=6.19, p<.001, d_{s}=1.180$, $95 \%$ CI $[60,117]$. Neutral distraction was $22 \mathrm{~ms}$ lower in the reward group $(M=34 \mathrm{~ms}, S D=$ $42 \mathrm{~ms})$ compared to the control group $(M=56 \mathrm{~ms}, S D=49 \mathrm{~ms}), t(105.6)=2.45, p=.016, d_{s}$ $=0.468,95 \%$ CI $[4,38]$. No other effects reached significance.

To ensure that the overall speeding of RTs in the reward group did not account for the effect of reward on distraction, a mixed-model ANOVA with distractor valence (negative, neutral, positive) as the within-subjects factor and reward group (reward, control) as the between-subjects factor was conducted on proportional distraction indices [((intact RT scrambled RT) / scrambled RT)*100], which reflect slowing on intact distractor trials as a percentage of RTs on scrambled trials, thus controlling for this overall group difference in RTs. Significant main effects of reward group, $F(1,108)=38.10, p<.001, \eta_{p}^{2}=.261, \omega^{2}=$ .252 , and distractor valence, $F(2,216)=50.80, p<.001, \eta_{p}{ }^{2}=.320, \omega^{2}=.290$, were qualified by a significant reward group $\times$ distractor valence interaction $F(2,216)=12.40, p<.001, \eta_{p}{ }^{2}$ $=.103, \omega^{2}=.066$. Mirroring the RT findings, negative distraction, $t(96.3)=5.33, p<.001, d_{s}$ $=1.016,95 \% \mathrm{CI}[7,16]$, and positive distraction, $t(105.6)=5.72, p<.001, d_{s}=1.091,95 \%$ CI $[7,15]$, were reduced by reward to a greater extent than neutral distraction, $t(105.6)=$ $2.06, p=.042, d_{s}=0.392,95 \%$ CI $[-0.08,5]$. The consistency of these findings with the RT analyses shows that the speeding of RTs in the reward group does not account for the benefit of reward on emotional distraction. 
Motivation and Emotional Distraction

\section{Accuracy}

Accuracy (proportion correct) was high overall $(M=.949, S D=.038)$. In line with the RT analysis, accuracy was analysed in a 2 (reward group: reward, control) $\times 2$ (distractor type: intact, scrambled) $\times 3$ (distractor valence: negative, neutral, positive) mixed-model ANOVA, with reward as the between-subjects factor and the other factors within-subjects. A main effect of reward showed that the reward group $(M=.940, S D=.042)$ was less accurate than the control group $(M=.958, S D=.030), F(1,108)=6.77, p=.011, \eta_{p}^{2}=.059, \omega^{2}=.050$, indicating a speed-accuracy tradeoff (see below). Main effects of distractor valence, $F(2$, $216)=19.64, p<.001, \eta_{p}{ }^{2}=.154, \omega^{2}=.145$, and distractor type, $F(1,108)=47.24, p<.001$, $\eta_{p}{ }^{2}=.304, \omega^{2}=.293$, were qualified by a distractor type $\times$ distractor valence interaction, $F(2$, 216) $=9.85, p<.001, \eta_{p}{ }^{2}=.084, \omega^{2}=.074$. Follow-up tests revealed that negative distraction was significantly greater than neutral distraction, $t(109)=2.66, p=.009, d_{z}=0.254,95 \% \mathrm{CI}$ $[.005, .032]$. Positive distraction was significantly greater than neutral distraction, $t(109)=$ $4.36, p<.001, d_{z}=0.416,95 \%$ CI $[.018, .047]$. Positive and negative distraction did not significantly differ from each other, $t(109)=1.84, p=.068, d_{z}=0.176,95 \%$ CI [-.001, .029]. No other accuracy effects reached significance. Thus, RT and accuracy results converge to reflect greater emotional than neutral distraction.

The main effect of reward group in both the RT and accuracy analyses indicates an overall speed-accuracy tradeoff; participants in the reward group were significantly faster $(139 \mathrm{~ms})$ but also significantly less accurate $(1.8 \%)$ than those in the control group. Reward did not influence accuracy measures of distraction, reflected by the non-significant reward group $\times$ distractor type interaction for the accuracy analysis, $F(1,108)=1.35, p=.247, \eta_{p}{ }^{2}=$ $.012, \omega^{2}=.002$, suggesting that a speed-accuracy tradeoff does not account for the effect of reward on RT distraction. To further ensure that a speed-accuracy tradeoff did not account for the effect of reward on emotional distraction, inverse efficiency scores (adjusting RTs for accuracy: [RT/proportion correct], see Bruyer \& Brysbaert, 2011) were analysed in a 2 (reward group: reward, control) $\times 2$ (distractor type: intact, scrambled) $\times 3$ (distractor valence: negative, neutral, positive) mixed-model ANOVA, with reward as the betweensubjects factor and the other factors within-subjects. Consistent with the RT analysis, the reward group $\times$ distractor type interaction, $F(1,108)=13.11, p<.001, \eta_{p}^{2}=.108, \omega^{2}=.039$; and reward group $\times$ distractor type $\times$ distractor valence interaction, $F(2,216)=6.76, p=$ $.001, \eta_{p}{ }^{2}=.059, \omega^{2}=.036$, remained significant. Negative distraction, $t(108)=2.84, p=.005$, 
Motivation and Emotional Distraction

$d_{s}=.541,95 \%$ CI $[20,116]$, and positive distraction, $t(104)=3.92, p<.001, d_{s}=0.748,95 \%$ CI $[49,148]$, were both attenuated by reward. In contrast to RT findings, neutral distraction did not significantly differ between groups, $t(103)=1.48, p=.143, d_{s}=0.281,95 \%$ CI [-6, 42]. All other significant effects mirrored those in the RT analyses. These findings demonstrate that a strategy of sacrificing accuracy for speed does not account for the effect of reward on emotional distraction.

\section{Pupil data}

Pupil area across the entire trial (baselined against the mean of the $200 \mathrm{~ms}$ pre-trial period) is shown separately for the control and reward groups in Figure 3.3.

\section{Whole Trial}
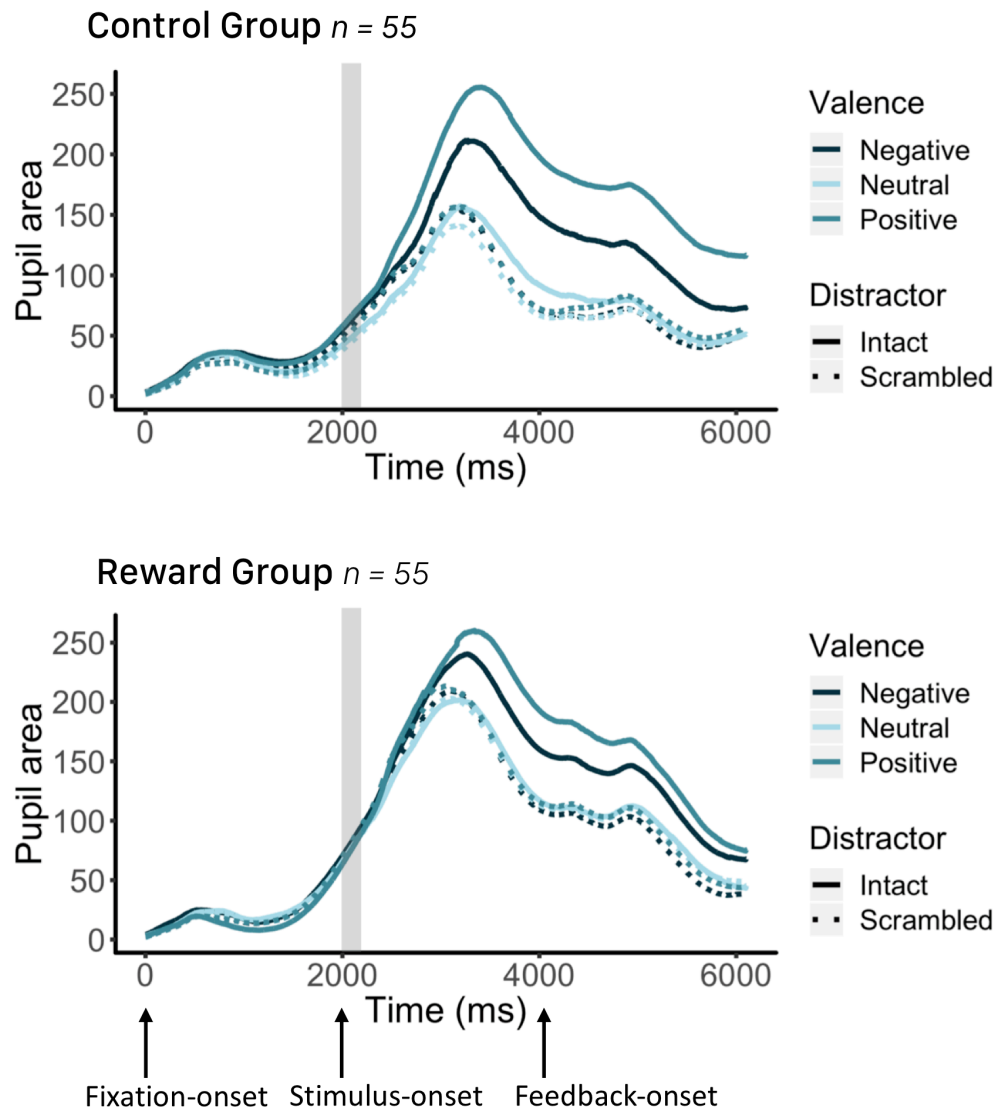

Figure 3.3. Mean pupil area (in arbitrary units) across the entire trial. Dilation is subtraction-baselined to the mean during the $200 \mathrm{~ms}$ time-window immediately preceding trial onset (on each trial) for the control group (top panel) and the reward group (bottom panel). Intact trials are represented by solid lines; scrambled images are represented by 
Motivation and Emotional Distraction

dotted lines. Distractor valence is represented by the different colour shades. Stimulus presentation (200 ms) is represented by the grey shaded area. Arrows indicate display-onset for fixation, stimulus, and feedback.

Proactive control pupil indices. See Table 3.2 for means and SDs for phasic and tonic proactive control indices. Note that the tonic means are all negative values; this is because tonic pupil area decreased across the experimental session in both groups, relative to the pre-experimental baseline block at the start of the session. This is not unusual in long experimental sessions. For simplicity, we will refer to differences in dilation between experimental conditions, however these should be interpreted in light of an overall pattern of constriction in experimental relative to baseline blocks.

Table 3.2. Mean pupil area in arbitrary units for preregistered tonic and phasic proactive timewindows, for each distractor valence condition, separately for control and reward groups. SDs are in brackets.

\begin{tabular}{lll}
\hline Valence Block & $\begin{array}{l}\text { Tonic } \\
\text { Proactive } \\
\text { Control }\end{array}$ & $\begin{array}{l}\text { Phasic } \\
\text { Proactive } \\
\text { Control }\end{array}$ \\
\hline Control Group & $-108(242)$ & $26(33)$ \\
Negative & $-121(240)$ & $23(26)$ \\
Neutral & $-90(244)$ & $24(30)$ \\
Positive & $-55(209)$ & $23(28)$ \\
Reward Group & $-89(228)$ & $22(26)$ \\
Negative & $-42(165)$ & $20(21)$ \\
Neutral & & \\
Positive &
\end{tabular}

To test hypotheses regarding how reward influences sustained and dynamic proactive control, separate mixed-model ANOVAs were conducted on the tonic and phasic proactive control indices. Distractor valence (negative, neutral, positive) was the within-subjects factor and reward group (reward, controls) was the between-subjects factor. Analysis of the tonic proactive indices (reflecting sustained dilation across blocks, see Figure 3.4) revealed that, as predicted, there was higher tonic dilation in emotional compared to neutral blocks, as reflected in the main effect of distractor valence, $F(2,216)=5.92, p=.003, \eta_{p}^{2}=0.052, \omega^{2}=$ .043 . 
Motivation and Emotional Distraction

Tonic Time-window
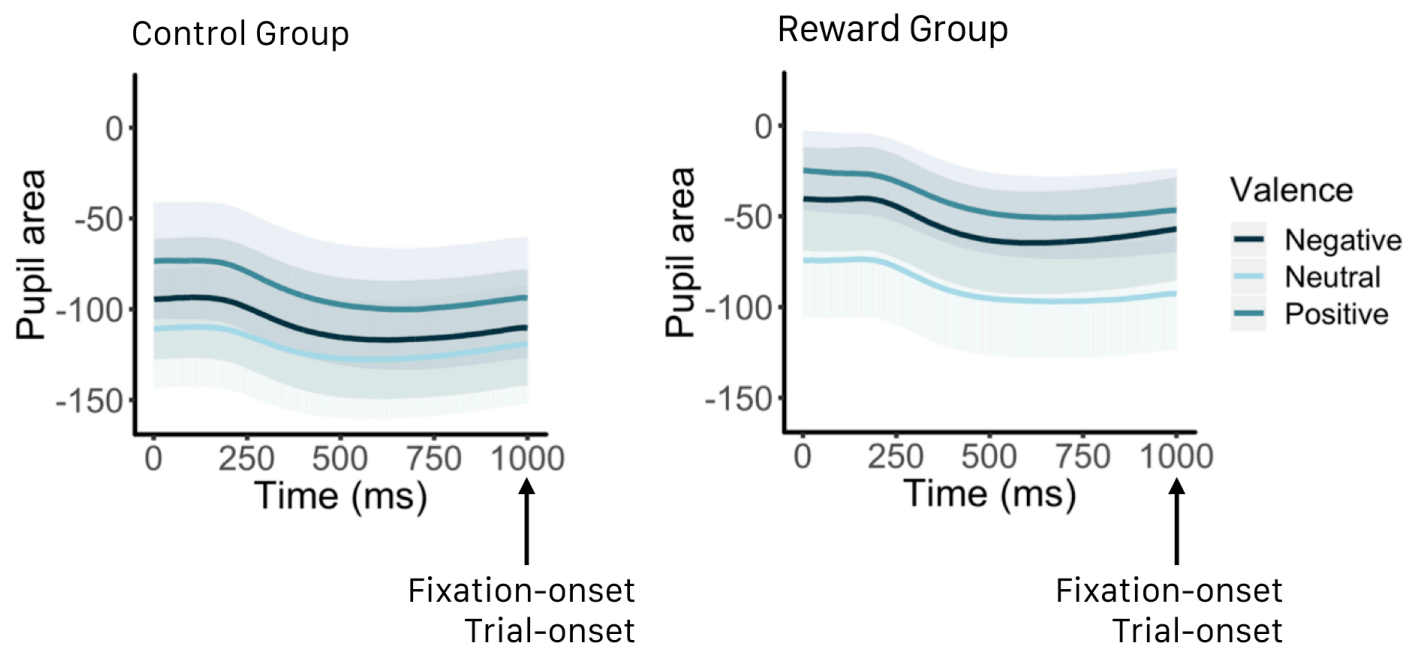

Figure 3.4. Mean pupil area (in arbitrary units) across the tonic time-window. Mean pupil area across the $1000 \mathrm{~ms}$ time-window preceding trial-onset, subtraction-baselined to the mean during the $200 \mathrm{~ms}$ time-window immediately preceding trial-onset in the baseline (scrambled-image only, non-reward) block. Overall, there was greater tonic dilation (or, rather, less constriction relative to baseline) on emotional compared to neutral blocks (with no difference between tonic dilation on positive and negative blocks). Tonic dilation was not modulated by reward group. Shaded error bands represent the standard error.

Planned comparisons showed that tonic pupil dilation in positive $(M=-66, S D=209)$ and negative $(M=-82, S D=227)$ blocks did not differ from each other, $t(109)=1.48, p=$ $.141, d_{z}=0.141,95 \%$ CI $[-37,5]$, and so positive and negative tonic dilation were collapsed together into emotional tonic dilation. Tonic dilation was greater on emotional $(M=-74, S D$ $=210)$ than neutral blocks $(M=-105, S D=234), t(109)=3.01, p=.003, d_{z}=0.287,95 \% \mathrm{CI}$ $[11,51]$, reflecting greater sustained arousal in an emotional context. Contrary to predictions, reward did not significantly increase tonic pupil dilation, $F(1,108)=1.21, p=.274, \eta_{p}{ }^{2}=$ $0.011, \omega^{2}=.002$ (although numerically tonic dilation was higher in the reward group compared to the control group; see Figure 3.4), and there was no reward group $\times$ distractor valence interaction, $F(2,216)=0.46, p=.629, \eta_{p}{ }^{2}=0.004, \omega^{2}<.001$. Thus, tonic dilation was greater in an emotional context, consistent with a shift to proactive control on emotional blocks (but see the Discussion of this chapter for an alternative explanation). However, tonic dilation was not modulated by reward group, suggesting that reward does not elicit sustained proactive control. 
Motivation and Emotional Distraction

Contrary to predictions, analysis of the phasic, dynamic, proactive control indices (reflecting preparation during the $2000 \mathrm{~ms}$ fixation period preceding stimulus-onset; see Figure 3.5) revealed no main effect of reward group, $F(1,108)=0.291, p=.591, \eta_{p}{ }^{2}=0.003$, $\omega^{2}<.001$, nor a reward group $\times$ distractor valence interaction, $F(2,216)=0.29, p=.748, n_{p}{ }^{2}$ $=0.003, \omega^{2}<.001$. Also contrary to predictions, there was no effect of distractor valence on phasic proactive control, $F(2,216)=0.70, p=.499, \eta_{p}{ }^{2}=0.006, \omega^{2}<.001$. Here we see no evidence that participants dynamically prepare differently for an upcoming trial as a function of either reward group or valence of a potential distractor.

\section{Pre-stimulus Phasic}

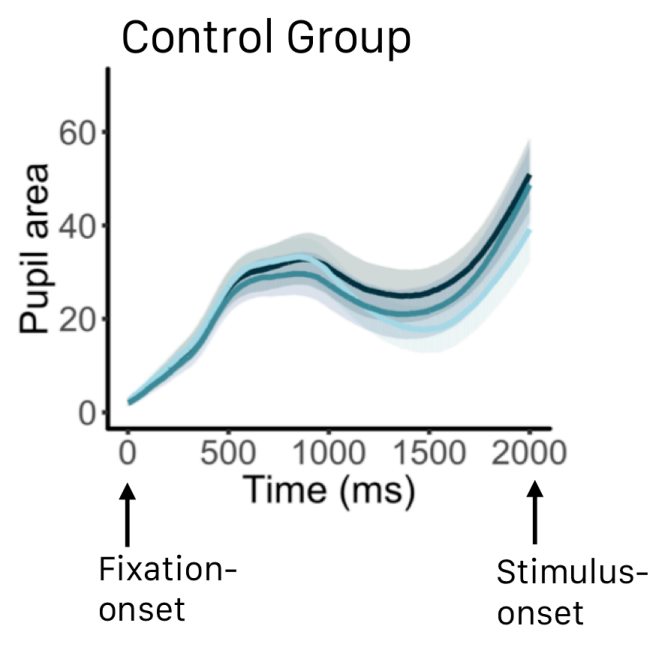

\section{Reward Group}

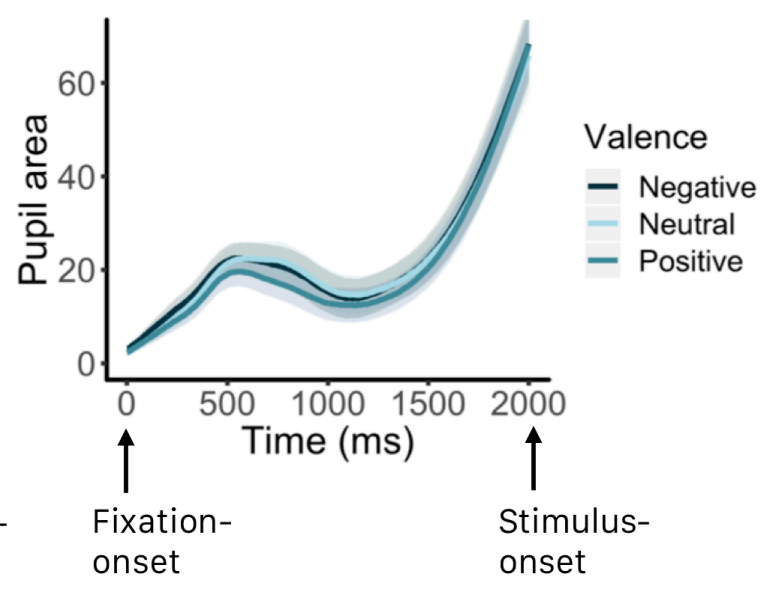

Figure 3.5. Mean pupil area (arbitrary units) across the pre-stimulus phasic time-window. Phasic proactive control is indexed by mean pupil area during the $2000 \mathrm{~ms}$ time-window preceding stimulus-onset, subtraction-baselined to the mean during the $200 \mathrm{~ms}$ time-window immediately preceding trial-onset. The preregistered analysis indicated no modulation of the mean phasic proactive control index (collapsed across the entire pre-stimulus time-window) by either distractor valence or reward group.

Post-stimulus pupil indices. To test hypotheses regarding the influence of reward on emotional and neutral distractor processing, an ANOVA was conducted on early and late post-stimulus pupil indices (which reflect intact distractor processing relative to scrambled distractor processing) with distractor valence (negative, neutral, positive) and time (early, late) as the within-subjects factors and reward group (reward, controls) as the betweensubjects factor. We had no specific predictions regarding differences between the two time- 
Motivation and Emotional Distraction

windows. See Table 3.3 for means for each group, split by valence and time, and Figure 3.6 for mean pupil area across the post-stimulus time-window.

Table 3.3. Comparisons of mean pupil area for the preregistered post-stimulus indices, for scrambled versus intact images, for each distractor valence condition, separately for control and reward groups. SDs are in brackets.

\begin{tabular}{|c|c|c|c|}
\hline $\begin{array}{l}\text { Valence } \\
\text { Block }\end{array}$ & $\begin{array}{l}\text { Scrambled } \\
\text { Trials }\end{array}$ & $\begin{array}{l}\text { Intact } \\
\text { Trials }\end{array}$ & Difference \\
\hline \multicolumn{4}{|c|}{ Control Group } \\
\hline \multicolumn{4}{|l|}{ Early } \\
\hline Negative & $67(57)$ & $116(85)$ & $49(50)$ \\
\hline Neutral & $67(57)$ & $79(65)$ & $12(33)$ \\
\hline Positive & $72(66)$ & $155(89)$ & $83(54)$ \\
\hline \multicolumn{4}{|l|}{ Late } \\
\hline Negative & $14(59)$ & $55(79)$ & 41 (59) \\
\hline Neutral & $25(71)$ & $27(77)$ & $2(51)$ \\
\hline Positive & $26(71)$ & $101(82)$ & $75(64)$ \\
\hline \multicolumn{4}{|c|}{$\underline{\text { Reward Group }}$} \\
\hline \multicolumn{4}{|l|}{ Early } \\
\hline Negative & $103(58)$ & $134(74)$ & $31(47)$ \\
\hline Neutral & $102(61)$ & $104(70)$ & $2(31)$ \\
\hline Positive & $106(63)$ & $165(82)$ & $59(51)$ \\
\hline \multicolumn{4}{|l|}{ Late } \\
\hline Negative & $20(62)$ & $57(87)$ & 37 (69) \\
\hline Neutral & $29(66)$ & $31(70)$ & $2(38)$ \\
\hline Positive & $25(79)$ & $83(93)$ & $58(67)$ \\
\hline
\end{tabular}

Note: Difference is [intact-scrambled]. $n=55$ per group. 


\section{Post-stimulus Phasic}
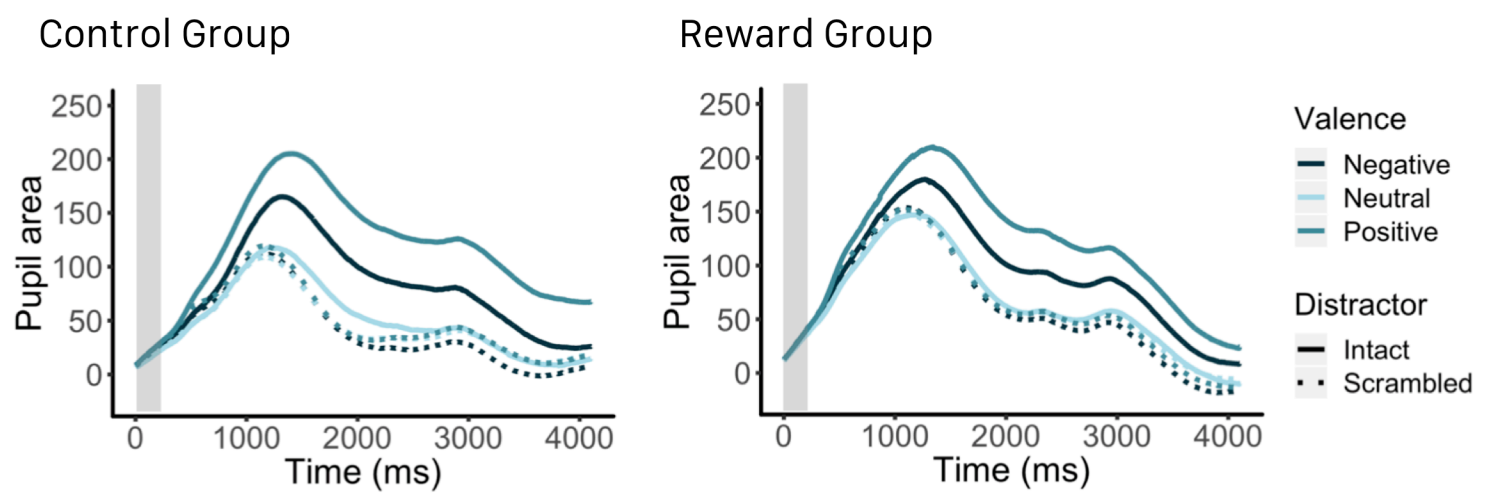

Figure 3.6. Mean pupil area (arbitrary units) across the 4100 ms post-stimulus timewindow. Pupil area across the entire post-stimulus period starting from stimulus-onset (subtraction-baselined to the mean $200 \mathrm{~ms}$ prior to stimulus-onset). Pupil area is split by valence, distractor type, and group (left: control group, right: reward group). Intact trials are represented by solid lines; scrambled images are represented by dotted lines. Distractor valence is represented by the different colour shades. Preregistered analyses of pupil indices from early and late post-stimulus time-windows showed modulation by both valence and reward group (see post-stimulus pupil indices).

The predicted main effect of distractor valence indicated that emotional distractors produced greater pupil dilation than neutral distractors, $F(2,216)=64.85, p<.001, \eta_{p}{ }^{2}=$ $0.375, \omega^{2}=.366$. Pupil dilation following positive distractors $(M=68, S D=57)$ was greater than following neutral distractors $(M=4, S D=36), t(109)=11.39, p<.001, d_{z}=1.086,95 \%$ CI $[53,75]$. Similarly, pupil dilation following negative distractors $(M=40, S D=53)$ was greater than following neutral distractors, $t(109)=6.40, p<.001, d_{z}=0.610,95 \%$ CI [24, 46]. Moreover, dilation in response to positive distractors was greater than for negative distractors, $t(109)=5.00, p<.001, d_{z}=0.476,95 \%$ CI $[17,40]$. The predicted reward group $\times$ distractor valence interaction was not significant, $F(2,216)=0.89, p=.413, \eta_{p}{ }^{2}=.008, \omega^{2}$ $<.001$, suggesting that reward did not reduce pupil dilation in response to emotional distractors to a greater extent than neutral distractors. Note that this differs from the behavioural finding that reward reduced emotional distraction to a greater extent than neutral distraction.

A trend toward the predicted main effect of reward group on post-stimulus pupil dilation, $F(1,108)=3.03, p=.084, \eta_{p}{ }^{2}=0.027, \omega^{2}=.018$, was qualified by a time $\times$ reward group interaction (which was not predicted), $F(1,108)=4.16, p=.044, \eta_{p}{ }^{2}=0.037, \omega^{2}=$ 
Motivation and Emotional Distraction

.027. To explore this interaction, two ANOVAs were conducted with distractor valence as the within-subjects factor and reward group as the between-subjects factor, separately for the early and late time-windows. This showed a significant effect of reward group on poststimulus pupil dilation in the early time window, $F(1,108)=6.93, p=.010, \eta_{p}^{2}=.060, \omega^{2}=$ .051 , but not in the late time-window, $F(1,108)=0.74, p=.392, \eta_{p}^{2}=.007, \omega^{2}<.001$. The timecourse of the effect of reward group on post-stimulus pupil dilation is further followed up in exploratory functional data analyses, below.

\section{Exploratory functional data analyses}

The following functional data analyses (FDA) are exploratory, that is, they were not preregistered. Averaging pupil dilation across pre (or post) specified (albeit arbitrary) timewindows is common in pupillometry studies (e.g., Bradley et al., 2008; Chiew \& Braver, 2013, 2014; Chevalier et al., 2015; Cohen et al., 2015; Henderson et al., 2014; Rondeel et al., 2015) and we chose to use indices calculated from pupil area in preregistered time-windows in keeping with these practices. However, reducing the rich $(1000 \mathrm{~Hz})$ pupil data to mean dilation across long windows we lose sensitivity to detect potential group differences in the timecourse of the implementation of control (see Sirois \& Brisson, 2014 for discussion of this issue). FDA (see Ramsay, Hooker, \& Graves, 2009; Ramsay \& Silverman, 1997; 2005) solves this problem; the discrete pupil samples are converted into smooth functions, for each condition and participant, and then inferential statistics are performed on the functions over time (described in more detail, below). The output of a functional $t$-test is a smooth function that defines the critical $t$-value (and associated $p$ value) at each time point. The advantage of this approach is that we can identify precisely if and when there are significant group differences (see Brisson et al., 2013; Jackson \& Sirois, 2009 for use of FDA with pupillometry data). We conducted FDA only on the phasic pre-stimulus response and the post-stimulus response, as these windows capture the dynamic changes in pupil dilation related to stimulus events. FDA is less appropriate for analyzing tonic dilation, where the goal is to identify stable difference in overall levels of dilation. Because the analysis was exploratory, we did not restrict it to the preregistered windows, and so FDA was applied to two time-windows, the first across the entire $2000 \mathrm{~ms}$ pre-stimulus window (from fixation onset to stimulus-onset, baselined to the $200 \mathrm{~ms}$ period immediately prior); and the second across the entire $4100 \mathrm{~ms}$ post-stimulus-onset window (from stimulus-onset until the end of the ITI, baselined to the $200 \mathrm{~ms}$ immediately prior). 
Motivation and Emotional Distraction

Following methods described by Ramsay and colleagues (1997; 2005; 2009), cubic Bsplines were created for each participant, for each valence, for each time-window, with a knot at each millisecond, and were smoothed by penalizing the second derivative. This creates a smooth curve that captures each participant's pupil response across time, for each valence. The B-splines for individual participants were then collapsed across distractor valence (because no interactions with valence were found in the preregistered analyses), and functional $t$-tests were conducted to examine the timecourse of group differences. To calculate the critical value of the test statistic, the labels of individual curves (for each participant, for each condition) were randomly shuffled and then the test statistic was calculated with the new labels. One thousand permutations were run, resulting in a null distribution (the pointwise critical value over time) based on the actual data. Actual data were then tested against the null distribution at each time point, to identify time-windows in which the reward and control groups significantly differ. See Appendix E for plots of the functional $t$-test output.

Exploratory pupil proactive control functional $t$-tests. Visual inspection of the pupil data suggests that by averaging pupil dilation across the $2000 \mathrm{~ms}$ time-window prior to stimulus-onset (our preregistered index of phasic proactive control) we missed important information about how proactive control unfolds across time, under conditions of reward relative to no reward (see Figure 3.5). Specifically, it appears that averaging across the timewindow might have disguised proactive control in the reward group that occurs close in time to stimulus-onset. To address this possibility, we conducted a functional $t$-test to determine whether (and when) this apparent group difference is significant. The timing of significant group differences are marked by asterisks in Figure 3.7. A significant group difference begins around $500 \mathrm{~ms}$ into the pre-stimulus period, extending until $1300 \mathrm{~ms}$ into the pre-stimulus period. However, contrary to predictions, this early difference is due to increased pupil dilation in the control group relative to the reward group, suggesting that the control group is implementing greater control than the reward group early in the fixation period. The second group difference occurs later, beginning around $1750 \mathrm{~ms}$ (250 ms prior to stimulus-onset) and extends through until stimulus-onset. This difference is in line with predictions, with the reward group showing greater pupil dilation, reflecting enhanced proactive control. Indeed, given that the reward group showed less dilation than the control group early in the pre-trial window, their eventual greater dilation reflects a steep gradient in dilation just before stimulus onset. The functional $t$-test reveals that the control and reward groups prepared to ignore the distractors over different timecourses. Specifically, the control group exerted effort 
Motivation and Emotional Distraction

earlier, and less intensely, with a small boost of control close to stimulus-onset. In contrast, the reward group prepared later, but more intensely, with a large dynamic boost of control close in time to stimulus-onset.

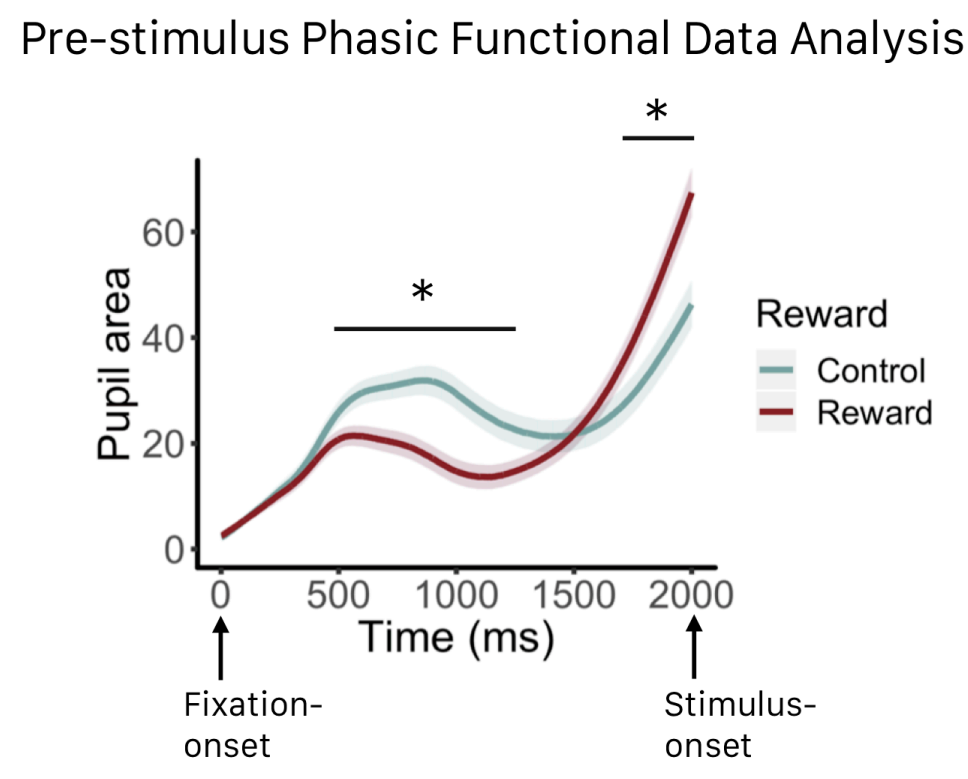

Figure 3.7. Functional data analysis of mean pupil area (arbitrary units) across the prestimulus phasic time-window. Phasic pupil dilation collapsed by valence during the prestimulus 2000 ms time-window. Significant group differences from the functional t-test are indicated by the horizontal lines and asterisks. Shaded error bands represent the standard error.

Exploratory post-stimulus pupil functional $t$-tests. In the preregistered poststimulus pupil ANOVA we found a trend toward the predicted main effect of reward group on post-stimulus pupil dilation, which was qualified by a time $\times$ reward group interaction. This interaction suggests an effect of reward group on pupil dilation elicited by the intact (relative to scrambled) distractors during some but not all of the post-stimulus time-period. To discover precisely when this group difference was significant, a functional $t$-test was performed on the [intact - scrambled] difference in pupil area across the $4100 \mathrm{~ms}$ timewindow, beginning at stimulus-onset and extending to the end of the inter-trial-interval period (subtraction-baselined to the $200 \mathrm{~ms}$ time-window immediately preceding stimulusonset) comparing pupil area between the reward and control groups. This time-window encompasses both the early and late preregistered post-stimulus phasic pupil indices, as well as the $200 \mathrm{~ms}$ stimulus-presentation and the $300 \mathrm{~ms}$ post stimulus-offset periods which were not included in the preregistered time-windows. The timing of significant group differences 
Motivation and Emotional Distraction

(extending from around 1000 to $2400 \mathrm{~ms}$ post-stimulus) are marked by asterisks in Figure 3.8A.

A. Post-stimulus Phasic Functional Data Analysis

Intact and Scrambled trials

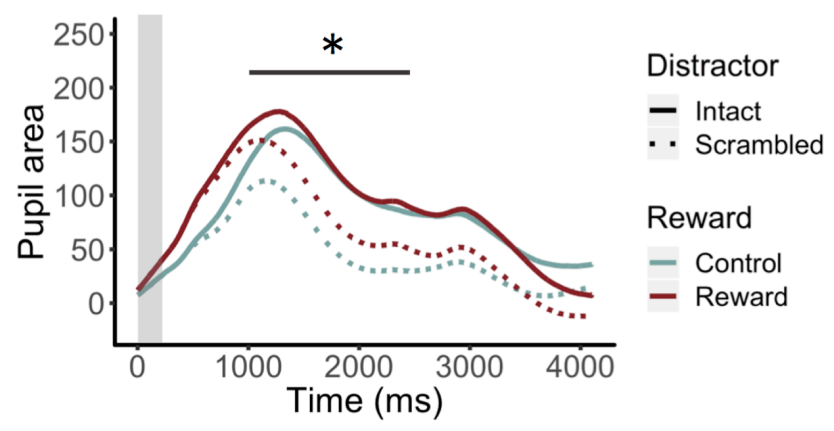

B. Post-stimulus Phasic Functional Data Analysis

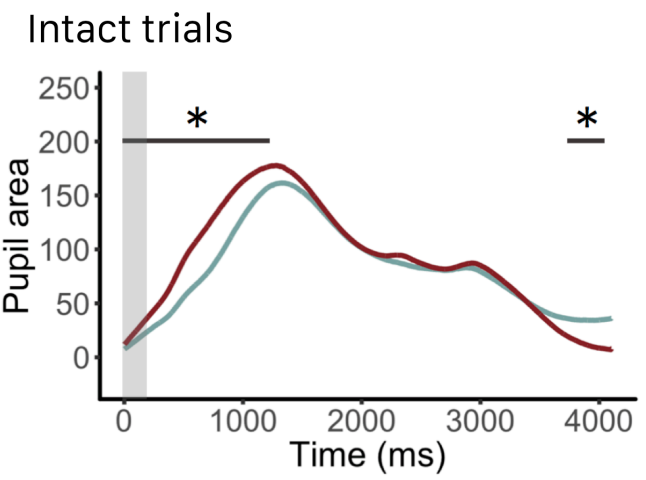

Scrambled trials

Figure 3.8. Functional data analysis of mean pupil area (arbitrary units) across the 4100 ms post-stimulus time-window. Panel A. Pupil dilation, collapsed across valence, split by distractor type and reward group. Intact trials are represented by solid lines, scrambled images are represented by dotted lines. Reward group is represented by the different colours. Significant group differences for the [intact-scrambled] difference from the functional t-test are indicated by the horizontal lines and asterisks. Panel B: Pupil dilation on intact trials (left), and scrambled trials (right), split by reward group. Significant group differences from the functional t-test are indicated by the horizontal lines and asterisks.

Inspection of Figure 3.6 suggests that, in addition to a reduction in the [intact scrambled] difference in pupil dilation, there appears to be a shift toward greater poststimulus pupil dilation overall in the reward group relative to the control group. To discover precisely when this group difference is significant, two functional $t$-tests were performed comparing the post-stimulus pupil dilation (separately for intact and scrambled trials, collapsed across distractor valence) over the entire $4100 \mathrm{~ms}$ post-stimulus time-window, to compare differences between the reward and control groups. For intact trials, there was 
Motivation and Emotional Distraction

greater pupil dilation in the reward group than in the control group for the first $1200 \mathrm{~ms}$ of the post-stimulus time-window (marked on Figure 3.8B, left graph). For scrambled trials, there was greater pupil dilation in the reward than the control group for the first $2550 \mathrm{~ms}$ of the post-stimulus time-window (marked on Figure 3.8B, right graph). Thus, while reward decreased pupil responses to intact relative to scrambled images (suggesting a reduction in sensitivity to the content of images), it did so by increasing pupil response to scrambles to a greater extent than to intact images in the early time window. I return to the implications of this finding in the Discussion.

Exploratory pupil-behaviour correlations. Nine exploratory correlations (see Figure 3.9) were calculated to examine how pupil dilation relates to behavioural responses.
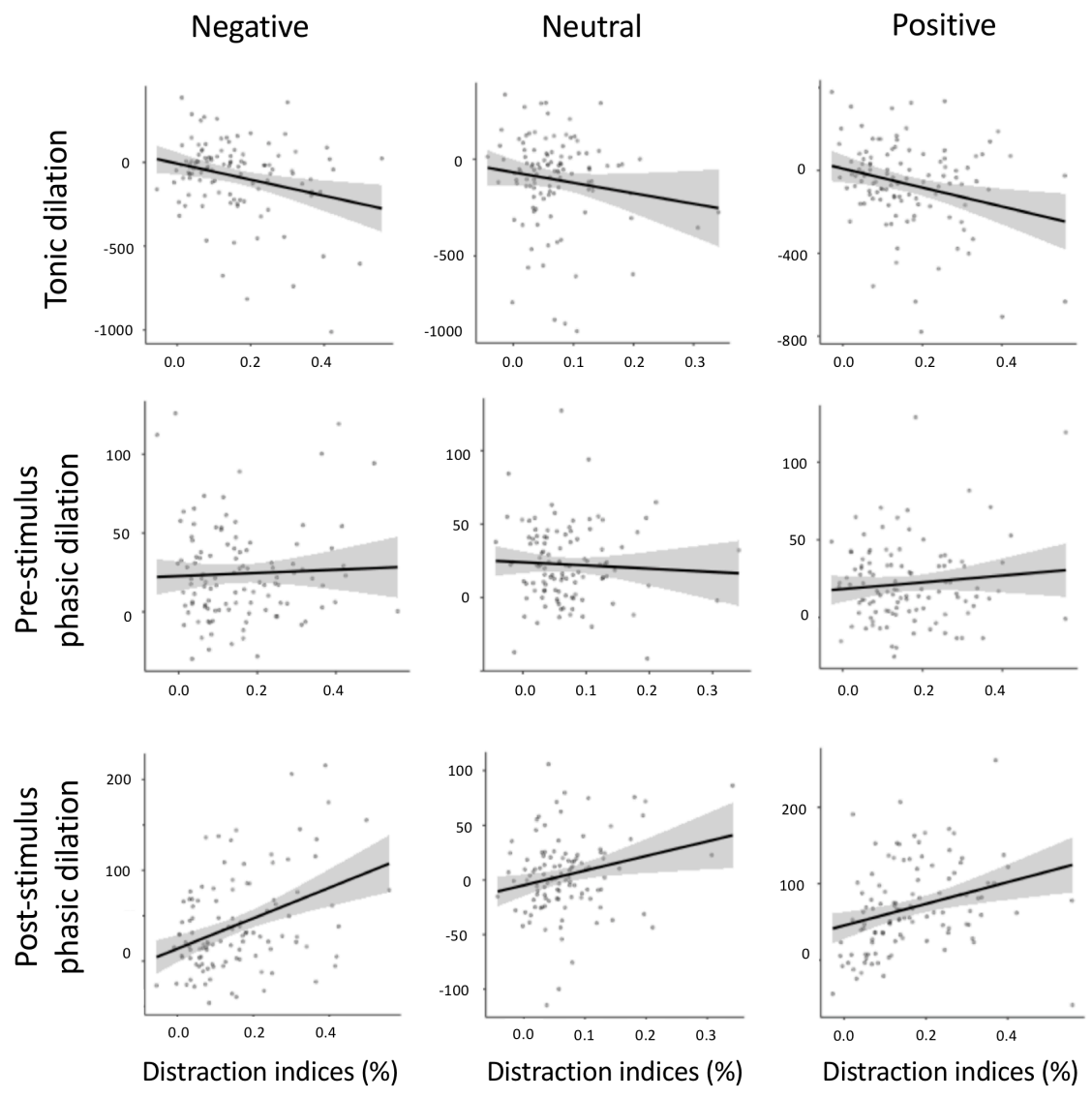

Figure 3.9. Correlations of pupil indices with behavioural distraction indices. Correlations between tonic (top row), pre-stimulus phasic (middle row), and post-stimulus phasic (bottom row) mean pupil indices and behavioural distraction indices on negative (left column), neutral (middle column) and positive (right column) blocks. Significant positive correlations were found for post-stimulus phasic pupil dilation [intact - scrambled difference] and behavioural distraction on positive and negative blocks. Significant negative correlations were found for tonic dilation and behavioural distraction for positive and negative blocks. 
Motivation and Emotional Distraction

If greater pupil dilation signals greater control, then pre-stimulus pupil dilation should be negatively correlated with behavioural measures of distraction. We examined the relationship between each mean pupil index (tonic, phasic pre-stimulus, phasic post-stimulus) and behavioural distraction indices, separately for each valence block (negative, neutral, positive). All significant correlations survived a Bonferroni correction (at alpha $=.05$, adjusted to .006 for nine tests) unless noted otherwise. First, to determine whether the phasic post-stimulus [intact - scrambled] difference in pupil dilation does actually reflect processing of the emotional distractors, we examined correlations between phasic post-stimulus dilation (averaged across the early and late time-windows) and behavioural distraction. Positive correlations were significant for all three valences: negative blocks, $r(108)=.396, p<.001$; positive blocks, $r(108)=.296, p=.002$; and neutral blocks, $r(108)=.232, p=.015$. Note however that the correlation for neutral blocks does not survive the Bonferroni correction. These findings validate the post-stimulus pupil dilation index as a measure of processing of the content of the emotional images, showing that the greater the pupil dilation following intact relative to scrambled images, the greater the behavioural distraction.

The tonic pupil index correlated negatively with emotional behavioural distraction, with greater tonic dilation on negative and positive blocks being associated with less negative, $r(108)=-.265, p=.005$, and positive, $r(108)=-.259, p=.006$, behavioural distraction, respectively. The correlation between tonic pupil dilation and behavioural distraction on positive blocks became non-significant with the Bonferroni correction ( $p=$ $.054)$. Tonic dilation on neutral blocks did not correlate with behavioural distraction, $r(108)=$ $-.144, p=.134$. These findings are consistent with the interpretation that increased tonic dilation is associated with increased sustained control when distractors are expected to be emotional.

However, phasic proactive pupil dilation (collapsed across the $2000 \mathrm{~ms}$ pre-stimulus time-window) did not correlate with negative, $r(108)=.042, p=.664$, positive, $r(108)=.099$, $p=.302$, or neutral, $r(108)=-.053, p=.584$, behavioural distraction.

\section{Discussion}

In the current experiment, as predicted, availability of performance-contingent reward decreased distraction by irrelevant images, particularly those that were emotional. The beneficial effect of reward on both positive and negative distraction replicates the finding from Experiment 1. Findings are also consistent with a small but growing body of evidence that motivation can enhance cognitive control, even when distractors are highly emotional 


\section{Motivation and Emotional Distraction}

(Padmala \& Pessoa, 2014; Padmala, et al., 2017). Mirroring the behavioural findings, relative to scrambled images, emotional distractors produced greater pupil dilation than neutral distractors, and dilation to intact distractors was reduced by reward. Furthermore, greater post-stimulus pupil dilation to emotional distractors predicted greater behavioural emotional distraction, establishing pupil dilation as a valid measure of emotional processing that has behavioural consequences. The main goal of this study was to investigate how reward influences the timing of implementation of control processes, by examining changes in pupil dilation over time. Pupil findings were consistent with reward-driven dynamic implementation of proactive control immediately prior to stimulus-onset, which then facilitated rapid reactive disengagement from emotional distractors.

Our primary research question concerned the timecourse by which motivation reduces distraction. Specifically, we tested the hypothesis that reward encourages a shift to an effective proactive control strategy to ignore emotional distractors. We considered two variants of proactive control indexed by pupil dilation: dynamic upregulation of control in preparation for an imminent distractor; and sustained implementation of control across entire blocks. Although we found no evidence that reward modulated sustained proactive control, exploratory analyses showed dynamic implementation of proactive control close in time to stimulus-onset in the reward group. Exploratory analyses also revealed that reward increased post-stimulus dilation (regardless of distractor type), suggesting upregulation of a reactive control mechanism. Together, these findings (examined in more detail below) are consistent with dynamic implementation of anticipatory control to more effectively disengage from distractors. We found no evidence that reward influences the timecourse of control differently for positive, negative, or neutral distractions.

We tested hypotheses using preregistered pupil indices (analysing baselined mean pupil area in specified time-windows) and exploratory analyses using a more data-driven approach to identify time periods in which pupil dilation was sensitive to reward. The tonic proactive control index (mean pupil area across the $1000 \mathrm{~ms}$ period prior to trial-onset) was greater in blocks in which distractors were emotional. It is difficult to determine whether this effect indicates greater sustained anticipatory control when emotional distractors were expected, or whether it is the result of emotional arousal carrying over from previously presented emotional distractors (which will have occurred on $25 \%$ of trials). One way to assess emotional carryover would be to analyse trials that followed an intact distractor separately from those that followed a scramble; however, we did not have sufficient trial numbers to make such an analysis robust. The correlations with behaviour, however, argue 
Motivation and Emotional Distraction

against emotional carryover, because greater tonic dilation on emotional blocks was associated with less emotional distraction. Thus, findings are consistent with the conclusion that tonic dilation is associated with enhanced sustained proactive control of emotional distraction, relative to neutral distraction. Emotional carryover, in contrast, would predict a positive relationship between tonic dilation and distraction on emotional blocks, because in the case of carryover, greater emotional arousal should be associated with greater emotional distraction.

Importantly, the tonic proactive control pupil index was not affected by reward. Findings therefore do not support the hypothesis that availability of reward encourages the use of a sustained proactive control strategy in this task. This finding contrasts with findings from Chiew and Braver (2013, 2014), who found that reward enhanced tonic pupil dilation, and behavioural proactive control, in a non-emotional context. Our null effect of reward on sustained proactive control could be due to insufficient power; tonic pupil data has high variability, and numerically our reward group did show higher tonic dilation than controls. However, the significantly greater tonic dilation on emotional than neutral blocks (discussed in the above paragraph) suggests that our study did not lack power to detect cognitive effects on tonic dilation. We should note that our task was very different to that of Chiew and Braver, who implemented an AX-CPT (which measures response conflict in a non-emotional context), with a within-subjects reward manipulation. These differences could account for our contrasting findings: reward may influence control of response conflict differently than it does for stimulus conflict; or reward might influence control in neutral contexts differently than it does in emotional contexts.

The preregistered phasic proactive control pupil index (mean pupil area across the $2000 \mathrm{~ms}$ pre-stimulus window) was not modulated by valence, suggesting that similar dynamic proactive mechanisms were engaged whether emotional or neutral distractors were expected. Preregistered analyses also revealed no influence of reward on phasic proactive control. However, the exploratory functional $t$-test showed a significant effect of reward, which arose because the timecourse of preparatory phasic pupil dilation differed between reward conditions. The test revealed (see Figure 3.7) an early window (from around $500-$ $1300 \mathrm{~ms}$ post fixation-onset) in which phasic pupil dilation was greater in control than in reward participants, and a late window (extending from around $1750 \mathrm{~ms}$ after fixation-onset and continuing until stimulus-onset) in which phasic pupil dilation was greater - and rapidly increasing - in reward than in control participants. This pattern suggests that the control group exerted proactive control earlier than the reward group. In contrast, the reward group 


\section{Motivation and Emotional Distraction}

exerted a sharp "burst" of proactive control closer in time to stimulus-onset. The time-lag between the onset of implementation of cognitive effort and an increase in pupil dilation is not precisely known, and may depend on the specific processes involved. But, in monkeys, single-cell recordings of activation in the LC evoke peak pupil dilation approximately $300 \mathrm{~ms}$ post-LC activation (Joshi et al., 2016). Although we do not know the exact lag between onset of proactive control and increases to pupil dilation (which means we do not know the precise time when rewarded participants initiated their increase in control), the fact that the rewardelicited pupil dilation occurs pre-stimulus at all indicates that it is indeed anticipatory. Our finding that reward dynamically elicits phasic proactive control is consistent with the effect of reward in non-emotional contexts (Chiew \& Braver, 2013, 2014), and indeed reward did not interact with valence in any of our pre-stimulus analyses. Thus, participants did not adjust their use of dynamic proactive control depending on the expected valence of the distractors.

Turning to the post-stimulus period, in both the preregistered and exploratory analyses, pupil dilation was modulated by both emotion and reward. Our preregistered analyses used indices of phasic post-stimulus pupil dilation (mean pupil response to intact distractors relative to scrambles), to assess processing of the content of distractor images while controlling for low-level visual features. Although behavioural distraction and the pupil response to distractors were broadly similar (both were greater for emotional than for neutral distractors, and both were reduced by reward), there were some inconsistencies in the effects of valence. Pupil dilation was greater following positive (erotic) distractors than following negative (body mutilation) distractors, which both produced greater dilation than neutral distractors. In contrast, behavioural distraction from positive and negative images did not differ (see also Experiment 1). The valence effect in the pupil response is consistent with findings from Henderson and colleagues (2014) showing greater pupil dilation to erotic images relative to negative images, even when they are matched for subjective ratings of arousal. Behaviourally (replicating Experiment 1), reward produced greater attenuation of distraction in emotional blocks than in neutral blocks, which may be consistent with reward altering the motivational competition between emotional distractors and the letter targets (discussed in more detail in Chapter 5: General Discussion). However, this effect was not observed in the pupil index, which showed similar effects of reward on pupil responses to emotional and neutral distractors.

Interestingly, further exploratory FDA showed that the effect of reward on poststimulus dilation was driven primarily by greater dilation on trials with scrambled distractors (but to a lesser extent intact distractors) in rewarded participants compared to controls. This 


\section{Motivation and Emotional Distraction}

suggests a general, control-driven increase in post-stimulus dilation under reward. Separately examining the timecourse of group differences in dilation on intact trials, scrambled trials, and the [intact - scrambled] difference, allows us to make inferences regarding the respective influences of reward on control- and emotion-related pupil dilation. Reward was associated with greater pupil dilation for both scrambles and intact images (regardless of valence) right from stimulus onset (Figure 3.8B). However, the effect of reward on the [intact - scrambled] difference did not emerge until about $1000 \mathrm{~ms}$ later (Figure 3.8A). We suggest that the early effect of reward reflects upregulation of cognitive control that is implemented in the face of any distractor. The later effect of reward on the difference in dilation between intact and scrambled trials reflects reduced emotional processing of distractors, due to rapid implementation of control. The idea that there are separable cognitive and emotional influences on pupil dilation is not new. Dilation due to emotional arousal is thought to be under sympathetic control (Beatty \& Lucero-Wagoner, 2000; Bradley et al., 2008), activating the pupil's dilator muscle; whereas dilation due to cognitive effort is the result of parasympathetic inhibition of the constrictor muscle (Samuels \& Szabadi, 2008). Thus it is possible for cognitive control and emotional arousal to independently affect pupil size.

A similar dissociation between control-related and emotion-related pupil responses was recently described by Kinner and colleagues (2017). They observed pupil responses to neutral and negative images that participants viewed passively, or that they prepared to view under instruction to either upregulate or down-regulate their emotional response. Pupil dilation in an early time-window after image onset was enhanced in emotion-regulation conditions, regardless of whether the instruction required them to upregulate or downregulate their emotional response. It was not until a later time-window that responses diverged, becoming larger in the upregulation than in the down-regulation condition. Kinner and colleagues therefore argue that early pupil responses reflect a "cognitive" component, indexing the effort applied to both emotion regulation strategies, whereas later responses reflect an "emotional" component that indexes the emotional consequences of that regulation. As described above, our findings could be similarly interpreted as showing an early effect of reward on pupil dilation, independent of the nature of the distractor, which reflects effortful control; followed by a later effect of reward on pupil dilation, dependent on distractor content, which reflects an emotional response. Our finding that reward influenced poststimulus dilation was present from stimulus-onset, and thus - given the time-lag of the pupil response - may to some extent reflect proactive control processes; but it continues for over two seconds after stimulus-onset, and therefore must to some extent also reflect reactive 


\section{Motivation and Emotional Distraction}

control processes. Together, our pattern of pupil findings suggest that reward dynamically elicits proactive control to rapidly disengage from the distractors, thereby reducing the pupil's emotional response (discussed further in Chapter 5: General Discussion). Future research utilising other temporally-sensitive measures, such as electroencephalography, may yield further insights into the timecourse of reward-based modulation of control of emotional distraction.

Although a decomposition of the pupil response into a cognitive and an emotional component provides a parsimonious explanation of our data, we cannot rule out an alternative explanation: that anticipation of reward induces emotional arousal that is reflected in the pupil response (e.g., Bouret \& Richmond, 2015). At this point we can say that post-stimulus pupil responses were separately modulated by both reward and the emotional nature of the distractor. Manipulations that can tease apart the relative contributions of control and emotion on pupil dilation will be an important goal for future studies. We also note that analysis of the post-stimulus pupil response in the late time-window could be influenced by performance feedback (presented $2000 \mathrm{~ms}$ post stimulus-onset), which is more affectively positive in the reward group than in the control group ("you won a point!" on successful reward trials). However, group differences in the post-stimulus pupil response (revealed by both the preregistered analyses and the exploratory FDA) were almost exclusively limited to the timewindow prior to feedback presentation, and therefore the potential affective influence of feedback should not impact our findings and conclusions. Finally, it should be noted that our findings in support of dynamic changes in proactive control were observed in the exploratory FDA, and not in our preregistered analyses using pre-defined windows. These exploratory findings therefore require replication with a preregistered analysis plan.

This experiment shows the value of pupillometry - a non-invasive and relatively lowcost tool - to further understanding of both emotion and cognitive control. Our findings confirm that performance-contingent reward can reduce emotional distraction, and suggest that it does so by dynamically upregulating control just before the anticipated onset of a stimulus, and extending that control into the post-stimulus period.

In Experiments 1 and 2 (the current study), distractors were presented centrally, within the spatial locus of attention. It remains to be seen whether motivation can also facilitate control of emotional distraction when the distractors appear peripherally. The following chapter examines, in two experiments, whether reward reduces emotional distraction under conditions in which the distractors must capture attention away from centrally-presented targets in order to impair performance. 
Motivation and Emotional Distraction

Chapter 4. The influence of monetary and non-monetary reward on attentional capture by peripheral emotional distractors.

Data from this chapter are not yet published.

Open data, code and analyses for this chapter are available at https://osf.io/vxwd4/

In Experiments 1 and 2, reward reduced positive and negative distraction, suggesting that motivation can enhance control of emotional distractions. These findings extend the influence of motivation within the DMC framework (Braver, 2012; Braver et al., 2007) to include proactive control of potent emotional distractions. In these experiments, and those of Padmala and colleagues (Padmala \& Pessoa, 2014; Padmala et al., 2017) the distractors appeared centrally, within the spatial locus of attention - we instruct participants' to look at the central fixation cross at the beginning of each trial. But in real life, distractors often appear outside the current spatial locus of attention, capturing attention away from goalrelevant stimuli. Central distractors impair task performance to a greater extent than peripherally-presented distractors (Beck \& Lavie, 2005), because spatial attention presumably must be disengaged and shifted from central distractors in order to attend to flanking target stimuli. Indeed, pupil dilation findings from Experiment 2 are consistent with reward dynamically upregulating proactive control mechanisms to facilitate rapid reactive disengagement from the distractors. But this strategy may be particularly effective when distractors appear centrally, within the spatial locus of attention.

In contrast, peripheral distractors must capture attention away from centrallypresented target stimuli in order to impair performance (Forster \& Lavie, 2008; Theeuwes, 1991, 1994). Under these conditions, different control mechanisms may be available to reduce distraction that are not used when distractors appear within the current spatial locus of attention. For example, location-based suppression mechanisms may proactively prevent attentional capture (Cepeda, Cave, Bichot, \& Kim, 1998; Serences, Yantis, Culberson, \& Awh, 2004; Van der Stigchel \& Theeuwes, 2006). The signal suppression hypothesis (Sawaki \& Luck, 2010) posits that salient singleton distractors send an "attend to me" signal, which can be proactively suppressed via top-down mechanisms to prevent attentional capture. This occurs at least in part by suppressing distractor features (e.g., colour; Gaspelin \& Luck, 2018), but, if predictable, distractor location can also be suppressed. For example, processing at locations at which salient singleton distractors are expected to occur can be suppressed to below baseline levels (Gaspelin \& Luck, 2018). Increasing distractor frequency (a manipulation of proactive control) can prevent attentional capture by emotional distractors 
Motivation and Emotional Distraction

when they appear in peripheral locations (Grimshaw et al., 2018; Murphy et al., 2018). It is not known whether motivation can similarly prevent attentional capture by peripheral emotional distractors. Here, in two experiments, we examine whether motivation can enhance control of attentional capture by peripherally-presented emotional distractors, as it did for emotional distractors that appear within the spatial locus of attention (in Experiments 1 and 2).

\section{Experiment 3: Does monetary reward attenuate attentional capture by peripheral emotional images?}

In the current experiment we ask, does reward attenuate attentional capture by peripheral emotional distractors? Findings from our lab (Grimshaw et al., 2018; Murphy et al., 2018) show that another manipulation of control - increasing the frequency of distractors - does reduce attentional capture by emotional distractors when they appear in the periphery. Here we use a similar identification task as in the previous experiments of this thesis, but with central targets and peripherally-presented distractors (as in Grimshaw et al., 2018; Murphy et al., 2018). This paradigm was adapted from one used by Forster \& Lavie (2008) who showed that peripheral distractors can disrupt processing of central targets, even if they share no target features and appear in non-target locations - that is, they are entirely irrelevant to the task. Participants were instructed to ignore the positive, negative, and neutral distractors, which were randomly presented on $25 \%$ of trials, above or below the central letter targets. Again, participants were randomly allocated to the reward or control groups, to perform the distraction task with or without availability of performance-contingent monetary reward. In Experiments 1 and 2 we used a scrambled image baseline condition from which to measure distraction (slowing) by intact images. In contrast, in both experiments in this chapter, we used a distractor-absent baseline condition, in which no image appeared. Presenting distractors in the periphery creates smaller distraction effects than when they appear centrally (Beck \& Lavie, 2005), so a distractor-absent baseline was chosen to ensure that the distractor images would capture attention and impair performance. Distraction was therefore indexed as slowing on distractor-present relative to distractor-absent trials, within a block.

As in previous experiments, we expected emotional images (both positive and negative) to be more distracting than neutral images (a distractor presence $\times$ distractor valence interaction), and we expected reward to speed up performance (a main effect of reward group). Crucially, if motivation reduces attentional capture from distractor images by 
Motivation and Emotional Distraction

upregulating cognitive control, we predicted less distraction in the reward group than the control group (a reward group $\times$ distractor presence interaction). As in previous experiments, we had two alternative hypotheses regarding how reward might reduce emotional versus neutral distraction, both of which would be reflected by a reward group $\times$ distractor presence $\times$ distractor valence interaction. The first was that reward might reduce emotional distraction to a lesser degree than neutral distraction, because our attention is so biased toward emotional stimuli. The second was that reward might reduce emotional distraction to a greater degree than neutral distraction. If the mechanisms that control distraction from peripherally-presented images are similar to those that control distraction from centrallypresented images, we would expect to find support for the second hypothesis, as was found with centrally-presented images in Experiments 1 and 2.

\section{Method}

\section{Participants and Sample Size Determination}

A total of 66 participants were recruited from the community via flyers, emails, and social media posts. All participants were women, to reduce within-group variance, and to allow for use of images that were carefully calibrated for valence and arousal ratings, which differ across genders. One participant was excluded for achieving less than $75 \%$ accuracy overall, and less than $60 \%$ accuracy on a single block; no participants met the criteria of displaying distraction $4 S D$ s above or below the mean in any condition. Another participant was excluded due to a technical error during data collection. These two excluded participants were replaced, resulting in 64 participants (mean age 23 years; range 18-39 years, who were randomly split into the control and reward groups ( $n=32$ per group). The size of the effect of reward on emotional distraction in Experiment 1 was $d_{s}=0.519$, and for Experiment 2 was $d_{s}$ $=0.939$; thus the average was $d_{s}=0.729$. A sensitivity analysis using G*Power (Faul et al., 2007) determined that a sample size of 32 participants per group would detect an effect of reward on distraction greater than or equal to $d_{s}=0.711$ (alpha $=.05$, two-tailed), $80 \%$ of the time. Control participants understood they would receive a $\$ 15$ supermarket voucher for participating. Reward participants understood they could earn between $\$ 10$ and $\$ 20$, contingent on performance.

\section{Materials}

As in Experiment 1, participants in the same experimental condition were run in groups of one to four in a dimly-lit room in separate booths. The experiment was run using E- 
Motivation and Emotional Distraction

Prime 2.0 (Psychology Software Tools, Pittsburgh, PA, USA), on Dell Precision T1700 personal computers with 24 " monitors at $1920 \times 1080$ pixel resolution and a $120 \mathrm{~Hz}$ refresh rate. A chin-rest maintained a viewing distance of $57 \mathrm{~cm}$.

The 12 positive (erotic images of heterosexual couples), 12 negative (mutilation images), and 12 neutral images (people in everyday scenarios) were the same as were used in Experiments 1 and 2 (IAPS; Lang et al., 2008).

Participants completed the Behavioural Activation and Inhibition Scale (BIS/BAS; Carver \& White, 1994). Scores on the BAS drive subscale were used for exploratory analyses of the influence of individual differences in reward sensitivity on performance, which are not the focus of this thesis, so they are not reported here.

\section{Task and Procedure}

Figure 4.1 illustrates the distraction task.

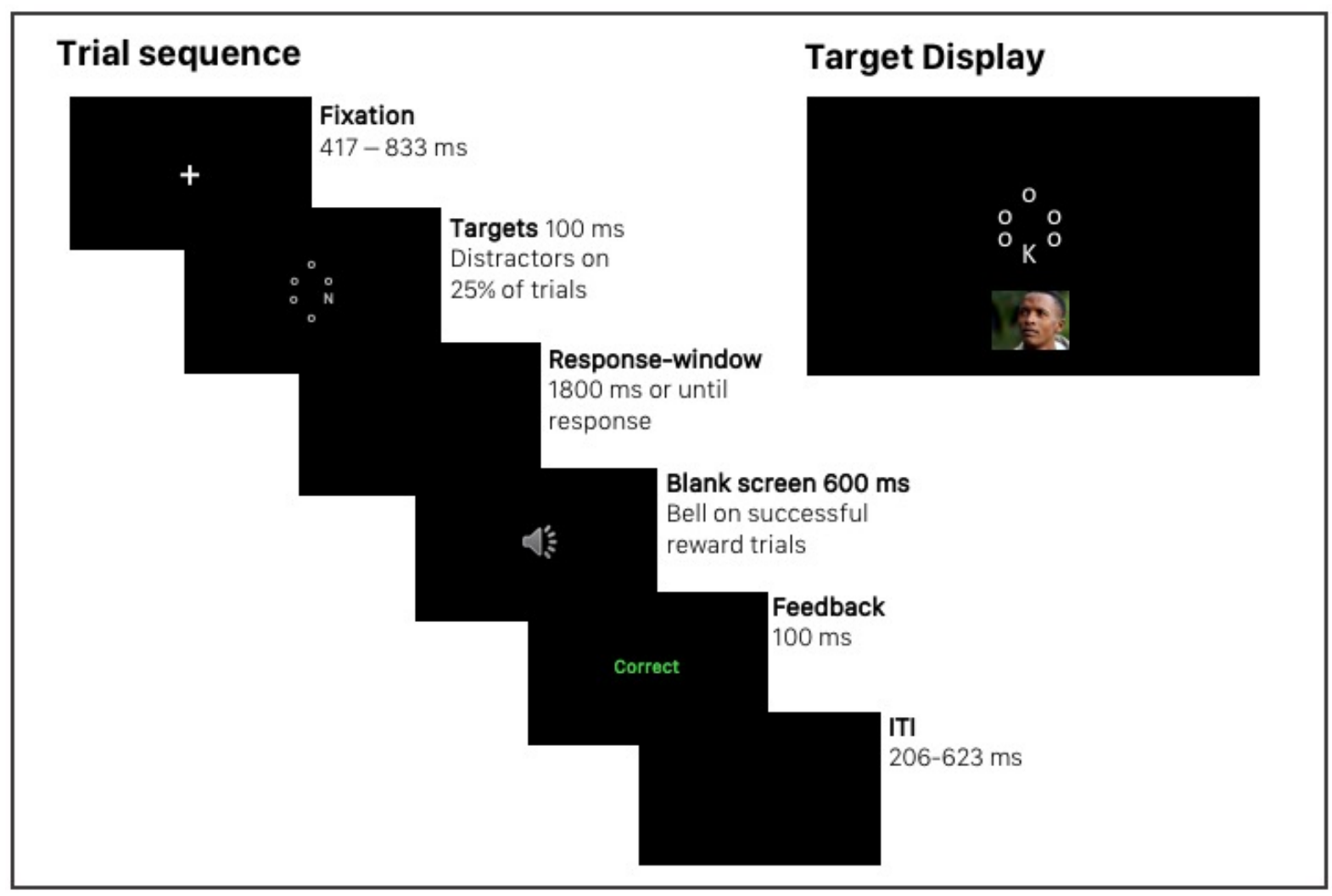

Figure 4.1. Trial sequence (left) for participants in the reward group, and the target display (right) for the peripheral distraction task in Experiments 3 and 4. The trial sequence (left) depicts a successful reward trial, with no distractor. The target display (right) depicts a neutral distractor-present trial. Distractors were presented on $25 \%$ of trials, and were blocked by valence (negative, neutral, positive). Participants in the control condition experienced the same trial sequence, but with no bell sound. For illustrative purposes, images are not to scale; the central part of the display is enlarged. 
Motivation and Emotional Distraction

The task was similar to that of Experiment 1 and 2, but with key differences to the target display configuration, target display presentation duration, and to the baseline condition. The reward manipulation, block structure, and distractor images were identical to those in Experiment 1.

For all participants, regardless of reward condition, a trial began with a central white fixation cross (jittered duration between 417-833 ms) presented on a black background, followed by a $100 \mathrm{~ms}$ target display, consisting of six white letters arranged in a circle around fixation (radius $1.75^{\circ}$ ). The target display was presented for $100 \mathrm{~ms}$ to prevent saccades toward the distractor images (which take longer than $100 \mathrm{~ms}$ ). On $25 \%$ of trials, a peripheral distractor image $\left(6.68^{\circ} \times 6.68^{\circ}\right.$, centred at $6.68^{\circ}$ eccentricity $)$ was presented simultaneously above or below the letter display. Five of the letters were o's $\left(0.22^{\circ} \times 0.22^{\circ}\right)$ and the target was $\mathrm{K}$ or $\mathrm{N}$ (height $0.67^{\circ}$, width $0.36^{\circ}$ ). As in Experiments 1 and 2, participants were instructed to ignore the images and respond as quickly and accurately as possible to indicate whether $\mathrm{K}$ or $\mathrm{N}$ was present, using keys 1 and 2 (counterbalanced across participants) on the number-pad with their dominant hand. Participants responded during a $1900 \mathrm{~ms}$ responsewindow from stimulus-offset. Failure to respond was recorded as an error. A 600 ms blank screen followed the response, followed by visual feedback (100 ms duration): "correct" in green, "incorrect" in red, or "please respond faster" in white (if no response was made within the response-window). A random inter-trial-interval ranged between 207-623 ms. Target letter and location were counterbalanced across trials, and trial order was randomised within blocks.

Participants completed 24 practice trials (six distractor-present, 18 distractor-absent) and a baseline block of 48 trials (12 distractor-present, 36 distractor-absent); these blocks contained scrambled images as distractors. These scrambled images were created from different intact images than those presented on experimental trials. To create scrambles, images were divided into $36 \times 36$ segments that were randomly recombined in PhotoScapev3.7.

As in Experiment 1, to limit valence carry-over effects, the experiment was split into two halves (super-blocks) of 192 trials (counterbalanced across participants). One superblock was split into blocks of negative and neutral distractors; the other super-block was split into blocks of positive and neutral distractors. A three-minute break separated the superblocks, to further limit valence carry-over effects. During the break, participants filled out a 
Motivation and Emotional Distraction

sudoku puzzle. Each super-block comprised two emotional and two neutral blocks (separated by self-timed breaks), presented in ABBA (counterbalanced) order. Within a block (48 trials; 12 distractor-present, 36 distractor-absent), all images were either negative, neutral, or positive, randomly mixed with distractor-absent trials. Each image was presented once in a block, although images were repeated (emotional images twice; neutral images four times) across the experiment to increase trial numbers to obtain stable measures of response times and distraction. This yielded four block orders, which were counterbalanced across participants. Total experiment session time was approximately 50 minutes.

Reward Condition. As in Experiment 1, after the baseline block, participants were informed about the potential to earn one point per trial if they were correct and faster than their median RT from the baseline block. Success was indicated by a pleasant sound (600 ms duration) immediately following response. Winning points led to achieving levels, and reaching a new level increased total earnings by $\$ 2.50$. Participants began on level 1 (total earnings: \$10). Level 2 required 91 points (total earnings: \$12.50). Ninety-one additional points were needed to ascend to each new level, up to level 5 (total earnings: \$20). During each break between blocks, point total and current level appeared above an animated coin display, along with mean RT and percent accuracy for the preceding block. Participants were encouraged to be fast and accurate to get to the next level. A warning was provided encouraging people to keep accuracy high if accuracy fell below 95\% in any block. Reward participants all earned between $\$ 15$ and $\$ 20(M=\$ 16.41)$.

Control Condition. Control participants completed the task as above, but were given $\$ 15$, not dependent on performance in return for participation. They received no auditory feedback on accuracy. During the breaks, instead of performance feedback and the coin animation, they saw 'please wait...' and instructions to take a break.

\section{Results and Discussion}

Mean correct RTs and accuracy for neutral distractor blocks did not differ across the two super-blocks (distractor-present and distractor-absent conditions; all $p$ 's > .361), so we collapsed the neutral distractor blocks in analyses. Mean correct RTs, RT distraction indices [distractor-present RT - distractor-absent RT], mean proportion correct scores, and accuracy distraction indices [distractor-absent proportion correct - distractor-present proportion correct] for each condition are presented in Table 4.1. RT distraction indices (reflecting slowing due to distractor images in each condition) are displayed in Figure 4.2. 
Motivation and Emotional Distraction

Table 4.1. Mean correct RTs (ms), accuracy (proportion correct), and distraction indices: [distractor-present RT - distractor-absent RT] and [distractor-absent proportion correct distractor-present proportion correct] for each distractor valence condition, separately for control and reward groups, for Experiment 3. SDs are in brackets.

\begin{tabular}{|c|c|c|c|c|c|}
\hline Valence & Distractor- & Distractor- & & & $95 \% \mathrm{CI}$ \\
\hline Block & absent & present & Distraction & $d_{z}$ & Low Upper \\
\hline
\end{tabular}

\section{$\underline{\text { Response Times }}$}

\section{Control Group}

\begin{tabular}{|c|c|c|c|c|c|c|}
\hline Negative & $526(59)$ & $555(82)$ & $29 * * *(36)$ & 0.809 & 16 & 42 \\
\hline Neutral & $531(60)$ & $555(74)$ & $24 * * *(25)$ & 0.958 & 15 & 33 \\
\hline Positive & $532(59)$ & $565(79)$ & $33 * * *(38)$ & 0.861 & 19 & 47 \\
\hline \multicolumn{7}{|c|}{$\underline{\text { Reward Group }}$} \\
\hline Negative & $472(50)$ & $486(68)$ & $14^{* *}(29)$ & 0.500 & 4 & \\
\hline Neutral & $474(55)$ & $481(54)$ & $7(22)$ & 0.314 & -1 & \\
\hline Positive & $473(54)$ & $489(64)$ & $16^{* * *}(24)$ & 0.709 & 8 & \\
\hline
\end{tabular}

\section{$\underline{\text { Proportion Correct }}$}

Control Group

\begin{tabular}{lcccccc} 
Negative & $.972(.026)$ & $.965(.042)$ & $.007(.047)$ & 0.148 & -.010 & .024 \\
Neutral & $.968(.025)$ & $.974(.022)$ & $-.006(.027)$ & 0.206 & -.015 & .004 \\
Positive & $.965(.027)$ & $.975(.033)$ & $-.010(.039)$ & 0.257 & -.024 & .004 \\
Reward Group & & & & & & \\
Negative & $.949(.055)$ & $.942(.058)$ & $.007(.059)$ & 0.128 & -.014 & .029 \\
Neutral & $.951(.045)$ & $.947(.043)$ & $.004(.029)$ & 0.156 & -.006 & .015 \\
Positive & $.945(.040)$ & $.956(.056)$ & $-.011(.048)$ & 0.236 & -.028 & .006 \\
\hline
\end{tabular}

Note: Effect sizes are Cohen's dz from paired comparisons of distractor-absent versus distractorpresent trials within conditions. ${ }^{* *} p<.010 .{ }^{* * *} p<.001 .95 \%$ confidence intervals surround the distraction scores, in ms and proportion correct. $n=32$ per group. 
Motivation and Emotional Distraction

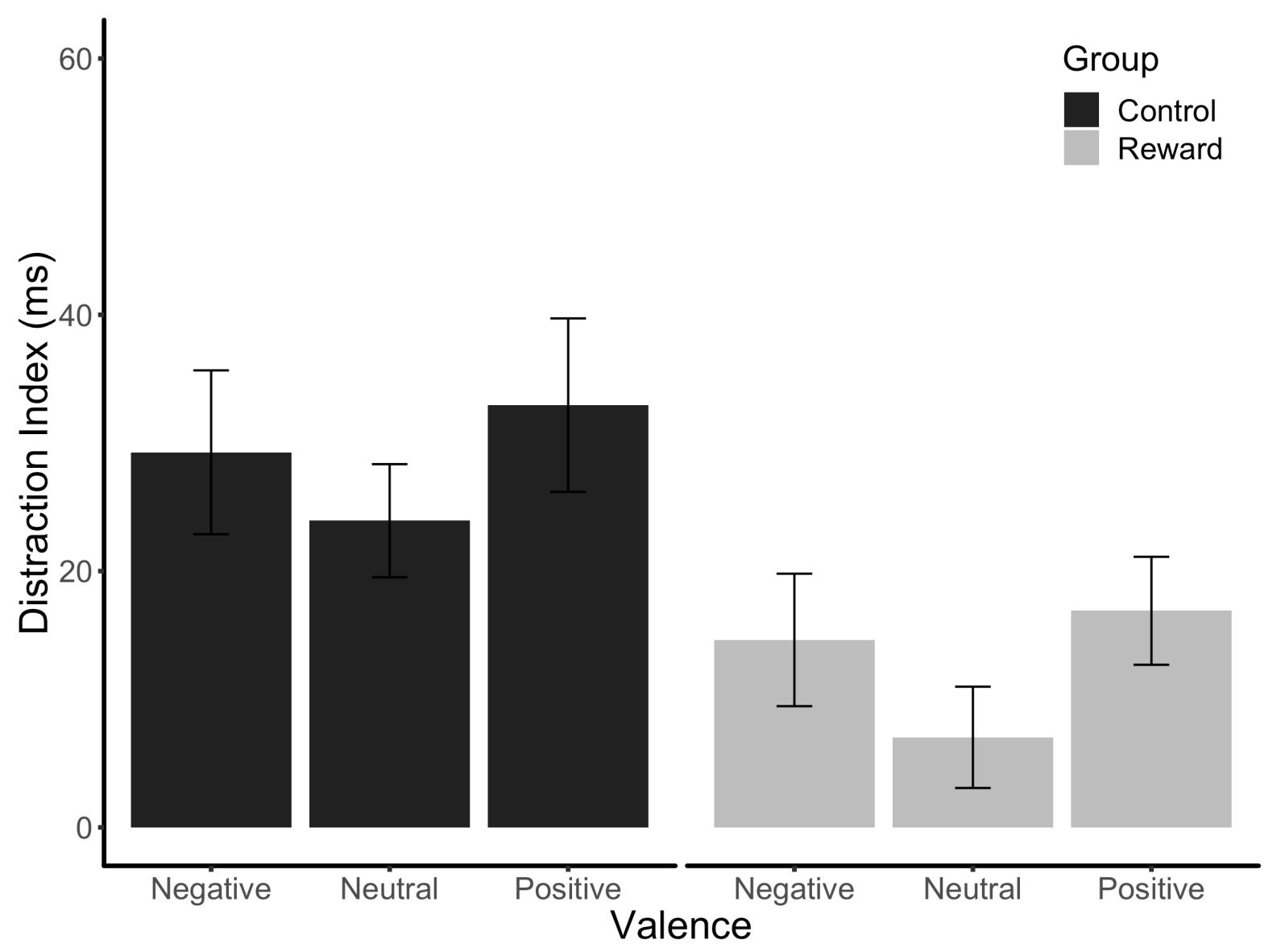

Figure 4.2. Mean behavioural distraction indices (in $\mathrm{ms}$ ) by reward group and valence for Experiment 3. Greater distraction [distractor present RT - distractor absent RT] was elicited by emotional than by neutral images. Neutral and emotional distraction were attenuated by reward. Error bars represent standard error of the mean.

\section{Response times}

To examine the influence of reward on task performance, correct RTs were analysed in a 2 (reward group: reward, control) $\times 2$ (distractor presence: present, absent) $\times 3$ (distractor valence: negative, neutral, positive) mixed-model ANOVA, with reward as the betweensubjects factor and the other factors within-subjects. The main effect of distractor presence, $F(1,62)=55.71, p<.001, \eta_{p}^{2}=.473, \omega^{2}=.431$, indicated that participants were $21 \mathrm{~ms}$ slower on distractor-present $(M=522 \mathrm{~ms}, S D=76 \mathrm{~ms})$ than distractor-absent $(M=501 \mathrm{~ms}$, $S D=61 \mathrm{~ms}$ ) trials. The predicted distractor presence $\times$ distractor valence interaction was not significant, $F(2,124)=2.55, p=.082, \eta_{p}{ }^{2}=.040, \omega^{2}=.024$. Despite this interaction not reaching significance, we conducted planned comparisons of distraction [distractor-present RT - distractor-absent RT], which showed that the marginal interaction was driven by 
Motivation and Emotional Distraction

significantly greater positive $(M=25 \mathrm{~ms}, S D=33 \mathrm{~ms})$ than neutral distraction $(M=16 \mathrm{~ms}$, $S D=25 \mathrm{~ms}), t(63)=2.38, p=.020, d_{z}=0.298,95 \% \mathrm{CI}[2,17]$. In contrast, negative distraction $(M=22 \mathrm{~ms}, S D=34 \mathrm{~ms})$ did not differ from neutral distraction, $t(63)=1.48, p=$ $.143, d_{z}=0.185,95 \%$ CI $[-2,15]$. Positive and negative distraction did not significantly differ, $t(63)=0.68, p=.500, d_{z}=0.085,95 \%$ CI $[-6,12]$.

The predicted main effect of reward group, $F(1,62)=18.60, p<.001, \eta_{p}{ }^{2}=.231, \omega^{2}$ $=.216$, reflected the fact that reward sped up responses overall (control group $M=544 \mathrm{~ms}$, $S D=65 \mathrm{~ms}$; reward group $M=479 \mathrm{~ms}, S D=54 \mathrm{~ms}$ ). Importantly, this main effect was qualified by the predicted reward group $\times$ distractor presence interaction, $F(1,62)=8.11, p=$ $.006, \eta_{p}{ }^{2}=.116, \omega^{2}=.056$. To explore this interaction, distraction indices (collapsed across the three valences) were compared between the control and reward groups; participants in the control group were significantly more distracted $(M=29 \mathrm{~ms}, S D=26 \mathrm{~ms})$ than those in the reward group $(M=13 \mathrm{~ms}, S D=18 \mathrm{~ms}), t(55.7)=2.85, p=.006, d_{s}=0.712$. The lack of a significant reward group $\times$ distractor presence $\times$ distractor valence interaction, $F(2,124)=$ $0.04, p=.965, \eta_{p}{ }^{2}=.001, \omega^{2}<.001$, indicates that (contrary to our prediction, and findings from Experiments 1 and 2) reward did not reduce emotional distraction to a greater extent than neutral distraction. No other RT effects reached significance.

To ensure that the overall speeding of RTs in the reward group did not account for the effect of reward on distraction, a mixed-model ANOVA with distractor valence (negative, neutral, positive) as the within-subjects factor and reward group (reward, control) as the between-subjects factor was conducted on proportional distraction indices [((distractorpresent RT - distractor-absent RT) / distractor-absent RT)*100], which reflect slowing on distractor-present trials as a percentage of RTs on distractor-absent trials, thus controlling for the overall group difference in RT. Consistent with the RT analyses, the main effect of reward group on proportional distraction was significant, $F(1,62)=6.75, p=.012, \eta_{p}{ }^{2}=$. $098, \omega^{2}=.082$. Also in line with the RT analyses, the effect of distractor valence was marginally significant, $F(2,124)=2.51, p=.086, \eta_{p}{ }^{2}=.039, \omega^{2}=.023$, and the reward group $\times$ distractor valence interaction was not significant, $F(2,124)=0.02, p=.980, \eta_{p}^{2}<$. $001, \omega^{2}<.001$. Thus, by mirroring the RT analyses, these findings show that the speeding of RTs in the reward group does not account for the benefit of reward on distraction.

\section{Accuracy}

Accuracy (proportion correct) was high overall $(M=.959, S D=.034)$. In parallel with the RT analysis, accuracy was analysed in a 2 (reward group: reward, control) $\times 2$ (distractor 
Motivation and Emotional Distraction

presence: present, absent) $\times 3$ (distractor valence: negative, neutral, positive) mixed-model ANOVA. A main effect of reward group $F(1,62)=7.13, p=.010, \eta_{p}^{2}=.103, \omega^{2}=.087$, indicated that the reward group $(M=.948, S D=.041)$ was significantly less accurate than the control group $(M=.970, S D=.019)$. The predicted distractor presence $\times$ distractor valence interaction did not reach significance, $F(2,124)=2.82, p=.063, \eta_{p}{ }^{2}=.044, \omega^{2}=.028$. Moreover, rather than reflecting our prediction of greater emotional than neutral distraction, this (non-significant) interaction was driven by greater negative $(M=.007, S D=.053)$ than positive distraction $(M=-.011, S D=.043), t(63)=2.12, p=.038, d_{z}=0.265,95 \%$ CI [.001, $.035]$. Neutral distraction $(M<-.001, S D=.028)$ did not differ from negative, $t(63)=1.01, p$ $=.316, d_{z}=0.127,95 \%$ CI [-.001, .023], or positive distraction, $t(63)=1.60, p=.114, d_{z}=$ $0.200,95 \%$ CI $[-.023, .003]$. No other main effects or interactions reached significance, including the predicted main effect of distractor presence, $F(1,62)=0.18, p=.676, \eta_{p}{ }^{2}=$ $.003, \omega^{2}<.001$.

The main effect of reward group in both RT and accuracy analyses reflects a speedaccuracy tradeoff; participants in the reward group were significantly $(65 \mathrm{~ms})$ faster but also significantly (2\%) less accurate than those in the control group. However, reward did not influence accuracy measures of distraction, reflected by the non-significant reward group $\times$ distractor presence interaction, $F(1,62)=0.25, p=.616, \eta_{p}{ }^{2}=.004, \omega^{2}<.001$, suggesting that a speed-accuracy tradeoff does not account for the effect of reward on RT distraction. To further ensure that the speed-accuracy tradeoff did not account for the effect of reward on distraction, we conducted a 2 (reward group: reward, control) $\times 2$ (distractor presence: present, absent) $\times 3$ (distractor valence: negative, neutral, positive) mixed-model ANOVA on inverse efficiency scores (adjusting RTs for accuracy: [RT/proportion correct]; see Bruyer \& Brysbaert, 2011). Main effects of reward group, $F(1,62)=13.40, p<.001, \eta_{p}{ }^{2}=.178, \omega^{2}=$ .163 , and distractor presence, $F(1,62)=31.26, p<.001, \eta_{p}{ }^{2}=.335, \omega^{2}=.309$, were qualified by the predicted but marginal reward group $\times$ distractor presence interaction, $F(1,62)=3.54$, $p=.065, \eta_{p}{ }^{2}=.054, \omega^{2}=.026$. To explore this marginal interaction, inverse efficiency distraction [distractor-present - distractor-absent], collapsed across the three valences, was compared between the control and reward groups. Participants in the control group were still marginally more distracted $(M=29 \mathrm{~ms}, S D=35 \mathrm{~ms})$ than those in the reward group $(M=14$ $\mathrm{ms}, S D=26 \mathrm{~ms}), t(56.8)=1.88, p=.065, d_{s}=0.470,95 \%$ CI $[-1,30]$. The fact that this effect of reward on distraction became marginal when inverse efficiency scores were examined indicates that a strategy of sacrificing accuracy for speed may account for some, 
Motivation and Emotional Distraction

but not all, of the effect of reward on distraction. I return to this point in Chapter 5: General Discussion.

In Experiment 3, performance-contingent monetary reward reduced distraction from peripherally-presented distractor images. Our finding suggests that motivation can enhance effective control of attentional capture by emotional distractors. This is in line with previous findings from our lab (Grimshaw et al., 2018; Murphy et al., 2018), who found that another manipulation of control (increasing distractor frequency) reduced positive and negative emotional distraction from peripherally-presented distractor images. The beneficial influence of reward on emotional distraction is also consistent with findings from Experiments 1 and 2, and those of Padmala and colleagues (Padmala \& Pessoa, 2014; Padmala et al., 2017), in which reward attenuated distraction from centrally-presented emotional distractors.

One concern with the present experiment is that emotional distractors were not significantly more disruptive then neutral distractors overall, as indicated by the nonsignificant distractor presence $\times$ distractor valence interaction $(p=.082)$. It was surprising that the control group was not significantly more distracted by emotional than neutral images (emotional distraction $M=31 \mathrm{~ms}$, versus neutral distraction $M=24 \mathrm{~ms} ; p=.205, d_{z}=0.229$ ). As a point of comparison, the same task produced $58 \mathrm{~ms}$ of emotional distraction and $24 \mathrm{~ms}$ of neutral distraction in Grimshaw et al., (2018). From Grimshaw et al. we derived an estimate of the emotional distraction effect size, $d_{z}=0.455$, which (at alpha $=.05$, two-tailed) requires a sample size of 40 participants to obtain $80 \%$ power ( $\mathrm{G}^{*}$ Power; Faul et al., 2007). In light of this, the lack of a significant emotional distraction effect seems less surprising in our control group of 32 participants. Peripherally-presented images produce less distraction than centrally-presented images. In retrospect, although the present study was sufficiently powered to detect the influence of reward on distraction, it may have been underpowered to detect significant emotional distraction in the control group. To foreshadow, we find a larger, statistically significant, emotional distraction effect in the control group of Experiment 4 (an identical condition to that of the control group in Experiment 3, but with $n=48$ ).

Contrary to findings from Experiment 1 and 2, there was no reward group $\times$ distractor presence $\times$ distractor valence interaction, indicating that the valence of a distractor did not impact the extent to which reward attenuated distraction. This may reflect a difference in how reward influences emotional versus neutral distraction when the distractors are presented centrally (as in Experiments 1 and 2) versus peripherally (as in the current experiment). However, this is unlikely as, to foreshadow again, we do observe the same reward group $\times$ 
Motivation and Emotional Distraction

distractor presence $\times$ distractor valence interaction in Experiment 4 , as we also found in Experiments 1 and 2. It therefore seems more likely that there was insufficient power in this experiment to detect the three-way interaction, and it is premature to conclude that different mechanisms mediate control of central versus peripheral distractors.

\section{Experiment 4: Does non-monetary reward attenuate attentional capture by peripheral emotional images?}

Experiment 3 showed that motivation, elicited by performance-contingent monetary reward, enhances effective control of attentional capture by emotional and neutral distractors. In Experiment 4, we ask whether motivation can also be elicited by non-monetary reward to improve performance and reduce emotional distraction. In Experiment 4, participants were randomly allocated to the reward or control groups, to perform the same distraction task with or without availability of performance-contingent non-monetary reward. In the lab, task motivation is often manipulated with performance-contingent accumulation of points, representing monetary reward (Botvinick \& Braver, 2015; Yee \& Braver, 2018). But in real life, motivation can be influenced by all sorts of factors (e.g., a desire to meet our own standards or to impress others, the presence of time pressure, how much we enjoy the activity, availability of primary reinforcers like sex or food, etc.), not just by how much money is available. This experiment examines whether points alone, a purely symbolic, nonmonetary, secondary reinforcer, are sufficient to induce motivation-elicited benefits to control of emotional distraction.

A handful of studies have examined the influence of non-monetary reward, relative to monetary reward, on cognitive control in emotionally-neutral contexts. Dambacher et al. (2011) and Hübner and Schlösser (2010) had participants complete a flanker task in which they could earn points for fast and accurate performance. For one group of participants, points represented greater monetary earnings, for another group they were purely symbolic. In both studies they found that monetary reward led to better accuracy than non-monetary reward, but only when the payoff contingencies emphasised speed to a greater degree than accuracy. Shen and Chun (2011) found that a non-monetary reward manipulation, which encouraged competition between participants, was just as effective as monetary reward at enhancing performance on attentional tasks. However, none of these studies compared the effectiveness of non-monetary reward to a no reward condition, and so it is not clear how "rewarding" either of the conditions were. In the current study, we investigate the extent to 
Motivation and Emotional Distraction

which non-monetary reward (relative to no reward) may be effective at motivating people to use a more effective cognitive control strategy to ignore emotional distractions.

As in Experiment 3, we expected emotional images (both positive and negative) to be more distracting than neutral images (a distractor valence $\times$ distractor presence interaction), and we expected reward to speed up performance (a main effect of reward group). Importantly, if non-monetary reward enhances effective control of attentional capture by distractors, we predicted less distraction in the reward group than the control group (a reward group $\times$ distractor presence interaction). As in previous experiments, we had two alternative hypotheses regarding how reward might reduce emotional versus neutral distraction, both of which would be reflected by a reward group $\times$ distractor presence $\times$ distractor valence interaction. The first was that reward might reduce emotional distraction to a lesser degree than neutral distraction, because our attention is so biased toward emotional stimuli. The second was that reward might reduce emotional distraction to a greater degree than neutral distraction. If the mechanisms that control distraction from peripherally-presented images are similar to those that control distraction from centrally-presented images, we would expect to find support for the second hypothesis, as was found with centrally-presented images in Experiments 1 and 2. Hypotheses, predictions, and analyses for Experiment 4 were preregistered $^{5}$ on aspredicted.org (link: http://aspredicted.org/blind.php? $\mathrm{x}=\mathrm{fd} 6 \mathrm{ig} 3$; see Appendix F).

\section{Method}

\section{Participants and Sample Size Determination}

Given concerns about statistical power to find an emotional distraction effect in Experiment 3, we increased the sample size in Experiment 4. A total of one-hundred female first year psychology students at Victoria University of Wellington completed the experiment. Two participants met the exclusion criteria of achieving less than $75 \%$ accuracy overall and less than $60 \%$ accuracy on a single block; two participants met the exclusion criteria of displaying distraction $4 S D$ s above the mean in one condition. These four participants were replaced, leaving a total of 96 participants in the sample (mean age 18.91, range 18-29 years) in the sample, who were randomly split into two groups of 48 . A

\footnotetext{
${ }^{5}$ Here, the only deviation from the preregistration is that the two one-sided equivalence tests (Lakens, 2017) examining whether reward similarly influenced positive and negative distraction are not presented. The tests were not conclusive in either direction, and therefore did not add anything to the findings or conclusions.
} 
Motivation and Emotional Distraction

sensitivity analysis using $G^{*}$ Power (Faul et al., 2007) determined that a sample size of 48 participants per group would detect an effect of reward on distraction greater than or equal to $d_{s}=0.578$ (alpha $=.05$, two-tailed), $80 \%$ of the time, which is comparable to the observed effect size from Experiment $3\left(d_{s}=0.565\right)$.

\section{Materials, Task and Procedure}

The experiment was run on E Prime 3.0 (Psychology Software Tools, Pittsburgh, PA, USA). The materials, task, and procedure were identical to those of Experiment 3 except that, in the reward group, achieving levels did not translate into monetary reward. Notably - and in line with Experiments 1 and 3 - on successful reward trials (i.e., trials on which participants in the reward condition were correct, and faster than their individual RT deadline for reward) participants received positive feedback (the pleasant sound) immediately following their response, signalling that they had earned a point. Reward participants again received updates on their point total, current level, and accuracy performance between blocks. Importantly (in contrast to all other experiments in this thesis), the accumulation of points, and achievement of levels had no monetary value. Instead, all participants received course credit in an introductory psychology course for their participation.

\section{Results and Discussion}

The following predictions and analyses were preregistered unless otherwise noted. Correct RTs and accuracy for neutral distractor blocks did not differ across the two superblocks (distractor-present and distractor-absent conditions; $p$ 's > .201), so we collapsed the neutral distractor blocks in analyses. Mean correct RTs, RT distraction indices, mean proportion correct scores, and accuracy distraction indices for each condition are presented in Table 4.2. RT distraction indices for each condition are displayed in Figure 4.1. 
Motivation and Emotional Distraction

Table 4.2. Mean correct RTs (ms), accuracy (proportion correct), and distraction indices: [distractor-present RT - distractor-absent RT] and [distractor-absent proportion correct distractor-present proportion correct] for each distractor valence condition, separately for control and reward groups, for Experiment 4. SDs are in brackets.

\begin{tabular}{llllcl}
\hline $\begin{array}{l}\text { Valence } \\
\text { Block }\end{array}$ & $\begin{array}{l}\text { Distractor- } \\
\text { absent }\end{array}$ & $\begin{array}{l}\text { Distractor- } \\
\text { present }\end{array}$ & Distraction & $d_{z}$ & \multicolumn{2}{c}{$\begin{array}{c}\text { 95\% CI } \\
\text { Lower }\end{array}$} \\
\hline
\end{tabular}

$\underline{\text { Response Times }}$

\section{Control Group}

\begin{tabular}{lllllll} 
Negative & $553(67)$ & $584(85)$ & $31^{* * *(40)}$ & 0.774 & 19 & 43 \\
Neutral & $557(64)$ & $570(74)$ & $13^{* *(29)}$ & 0.433 & 4 & 21 \\
Positive & $559(60)$ & $606(103)$ & $47^{* * *(64)}$ & 0.747 & 29 & 66 \\
\multicolumn{2}{l}{ Reward Group } & & & & & \\
Negative & $503(68)$ & $514(75)$ & $11^{* *(24)}$ & 0.447 & 4 & 18 \\
Neutral & $502(53)$ & $511(63)$ & $9 * *(19)$ & 0.473 & 4 & 14 \\
Positive & $507(65)$ & $518(64)$ & $11^{* *(27)}$ & 0.425 & 4 & 19
\end{tabular}

\section{Proportion Correct}

\section{Control Group}

\begin{tabular}{lcccccc} 
Negative & $.958(.054)$ & $.962(.044)$ & $-.004(.045)$ & 0.098 & -.017 & .009 \\
Neutral & $.961(.038)$ & $.965(.043)$ & $-.004(.037)$ & 0.114 & -.015 & .006 \\
Positive & $.961(.050)$ & $.961(.050)$ & $.000(.038)$ & 0.011 & -.011 & .011 \\
Reward Group & & & & & & \\
Negative & $.930(.051)$ & $.941(.054)$ & $-.011(.052)$ & 0.215 & -.026 & .003 \\
Neutral & $.937(.043)$ & $.935(.047)$ & $.002(.042)$ & 0.037 & -.011 & .014 \\
Positive & $.940(.043)$ & $.934(.068)$ & $.006(.065)$ & 0.089 & -.013 & .025 \\
\hline
\end{tabular}

Note: Effect sizes are Cohen's $d_{z}$ from paired comparisons of distractor-absent versus distractorpresent trials within conditions. ${ }^{* *} p<.010 .{ }^{* * *} p<.001 .95 \%$ confidence intervals surround the distraction scores, in ms and proportion correct. $n=48$ per group. 
Motivation and Emotional Distraction

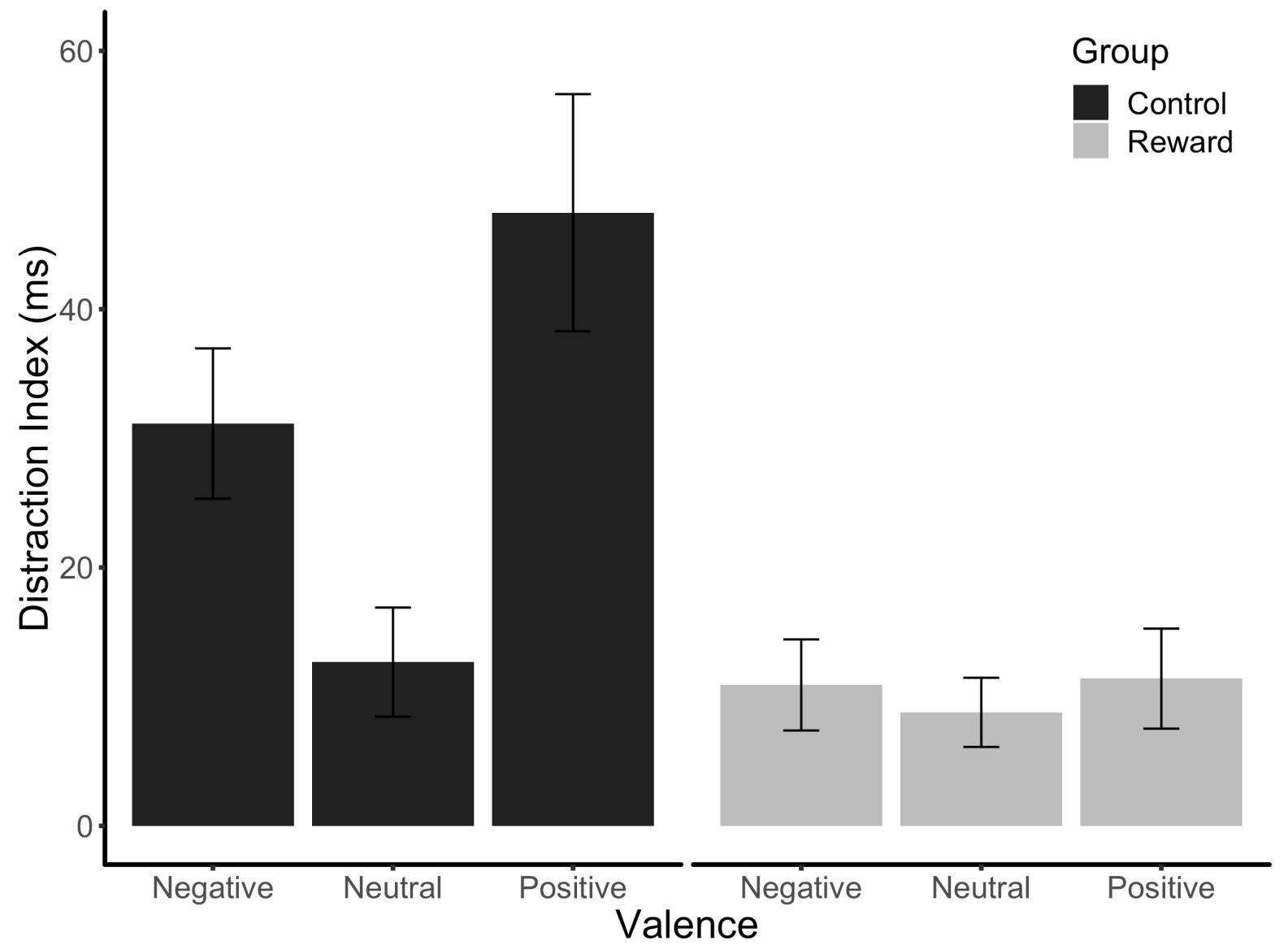

Figure 4.1. Mean behavioural distraction indices (in $\mathrm{ms}$ ) by reward group and valence for Experiment 4. Greater distraction (distractor present $R T$-distractor absent $R T$ ) was elicited by emotional than by neutral images. Emotional but not neutral distraction was attenuated by reward. Error bars represent standard error of the mean.

\section{Response times}

To examine the influence of reward on task performance, correct RTs were analysed in a 2 (reward group: reward, control) $\times 2$ (distractor presence: present, absent) $\times 3$ (distractor valence: negative, neutral, positive) mixed-model ANOVA, with reward as the betweensubjects factor and the other factors within-subjects. Main effects of valence, $F(1.67,156.92)$ $=6.78, p=.003, \eta_{p}{ }^{2}=.067, \omega^{2}=.056$, and distractor presence $F(1,94)=54.42, p<.001, \eta_{p}{ }^{2}$ $=.367, \omega^{2}=.329$, were qualified by the predicted distractor presence distractor valence interaction, $F(1.59,149.30)=8.92, p<.001, \eta_{p}{ }^{2}=.087, \omega^{2}=.072$, reflecting the fact that participants were more distracted by emotional images than by neutral images. Comparing distraction [distractor-present RT - distractor-absent RT] between valences showed that the interaction was driven by significantly greater positive $(M=29 \mathrm{~ms}, S D=52 \mathrm{~ms})$ than neutral distraction $(M=11 \mathrm{~ms}, S D=24 \mathrm{~ms}), t(95)=3.72, p<.001, d_{z}=0.380,95 \% \mathrm{CI}[9,29]$; and 
Motivation and Emotional Distraction

greater negative $(M=21 \mathrm{~ms}, S D=35 \mathrm{~ms})$ than neutral distraction, $t(95)=3.18, p=.002, d_{z}=$ $0.325,95 \% \mathrm{CI}[4,17]$. Positive and negative distraction did not significantly differ, $t(95)=$ $1.63, p=.108, d_{z}=0.166,95 \%$ CI $[-2,19]$.

The predicted main effect of reward group, $F(1,94)=21.50, p<.001, \eta_{p}{ }^{2}=.186, \omega^{2}$ $=.176$, (control group $M=571 \mathrm{~ms}, S D=69 \mathrm{~ms}$; reward group $M=509 \mathrm{~ms}, S D=62 \mathrm{~ms}$ ), was qualified by the predicted reward group $\times$ distractor presence interaction $F(1,94)=$ $13.17, p<.001, \eta_{p}{ }^{2}=.123, \omega^{2}=.075$. To explore this interaction, distraction indices were compared between the control and reward groups. Participants in the control group were significantly more distracted $(M=30 \mathrm{~ms}, S D=36 \mathrm{~ms})$ than those in the reward group $(M=$ $10 \mathrm{~ms}, S D=14 \mathrm{~ms}), t(60.5)=3.63, p<.001, d_{s}=0.710,95 \%$ CI $[9,31]$. Furthermore, the predicted reward group $\times$ distractor presence $\times$ distractor valence interaction, $F(2,188)=$ $6.58, p=.002, \eta_{p}^{2}=.065, \omega^{2}=.051$, was explored by comparing differences in distraction indices between groups, separately for each valence. Negative distraction was reduced in the reward group $(M=11 \mathrm{~ms}, S D=24 \mathrm{~ms})$ relative to the control group $(M=31 \mathrm{~ms}, S D=40$ $\mathrm{ms}), t(77.5)=2.98, p=.004, d_{s}=0.608,95 \% \mathrm{CI}[7,34]$; likewise, positive distraction was reduced in the reward group $(M=11 \mathrm{~ms}, S D=27 \mathrm{~ms})$ relative to the control group $(M=48$ $\mathrm{ms}, S D=64 \mathrm{~ms}), t(63.2)=3.62, p<.001, d_{s}=0.739,95 \% \mathrm{CI}[16,56]$; in contrast, neutral distraction did not differ between the reward $(M=9 \mathrm{~ms}, S D=19 \mathrm{~ms})$ and control $(M=13$ $\mathrm{ms}, S D=29 \mathrm{~ms}$ ) groups, $t(79.6)=0.78, p=.439, d_{s}=0.159,95 \%$ CI $[-6,14]$. No other RT effects reached significance.

To ensure that the overall speeding of RTs in the reward group did not account for the effect of reward on distraction, a mixed-model ANOVA with distractor valence (negative, neutral, positive) as the within-subjects factor and reward group (reward, control) as the between-subjects factor was conducted on proportional distraction indices [((distractorpresent RT - distractor-absent RT) / distractor-absent RT)*100], which reflect slowing on intact distractor trials as a percentage of RTs on distractor-absent trials, thus controlling for the overall group difference in RTs. The main effect of reward group on distraction remained significant, $F(1,94)=11.80, p<.001, \eta_{p}{ }^{2}=.112, \omega^{2}=.101$, as did the reward group $\times$ distractor valence interaction, $F(2,188)=5.66, p=.004, \eta_{p}{ }^{2}=.057, \omega^{2}=.042$. Thus, the fact that RTs were faster overall in the reward group relative to the control group did not account for the effect of reward on emotional distraction. 
Motivation and Emotional Distraction

\section{Accuracy}

Accuracy was high overall $(M=.949, S D=.040)$. In parallel to the RT analysis, accuracy (proportion correct) was analysed in a 2 (reward group: reward, control) $\times 2$ (distractor presence: present, absent) $\times 3$ (distractor valence: negative, neutral, positive) mixed-model ANOVA. A main effect of reward group $F(1,94)=10.60, p=.002, \eta_{p}{ }^{2}=.102$, $\omega^{2}=.091$, indicated that the reward group $(M=.936, S D=.038)$ was significantly less accurate that the control group $(M=.961, S D=.038)$. No other main effects or interactions reached significance, including the predicted main effect of distractor presence, $F(1,94)=$ $0.54, p=.462, \eta_{p}^{2}=.006, \omega^{2}<.001$, or the distractor presence $\times$ distractor valence interaction, $F(2,188)=1.22, p=.298, \eta_{p}^{2}=.013, \omega^{2}=.002$.

As in Experiment 3, the main effect of reward group in both the RT and accuracy analyses indicates an overall speed-accuracy tradeoff; participants in the reward group were significantly $(62 \mathrm{~ms})$ faster but also significantly less $(2.5 \%)$ accurate than those in the control group. Reward did not influence distraction as measured in accuracy, reflected by the non-significant reward group $\times$ distractor presence interaction, $F(1,94)=0.08, p=.780, \eta_{p}^{2}=$ $.001, \omega^{2}<.001$, suggesting that a speed-accuracy tradeoff does not account for the effect of reward on RT distraction.

To further ensure that the speed-accuracy tradeoff did not account for the effect of reward on distraction, we conducted a 2 (reward group: reward, control) $\times 2$ (distractor presence: present, absent) $\times 3$ (distractor valence: negative, neutral, positive) mixed-model ANOVA on inverse efficiency scores (adjusting RTs for accuracy: [RT/proportion correct]; see Bruyer \& Brysbaert, 2011). Main effects of reward group, $F(1,94)=13.00, p<.001, \eta_{p}{ }^{2}$ $=.122, \omega^{2}=.112$, distractor presence, $F(1,94)=37.84, p<.001, \eta_{p}{ }^{2}=.287, \omega^{2}=.263$, and distractor valence, $F(1.57,147.80)=5.10, p=.012, \eta_{p}{ }^{2}=.051, \omega^{2}=.041$, were qualified by the following 2 and 3 way interactions: first, a reward group $\times$ distractor presence interaction, $F(1,94)=7.00, p=.010, \eta_{p}{ }^{2}=.069, \omega^{2}=.043$; and second, a reward group $\times$ distractor presence $\times$ distractor valence interaction, $F(2,188)=3.26, p=.040, \eta_{p}^{2}=.034, \omega^{2}=.022$. As was done for RTs, this three-way interaction was explored by comparing group differences in inverse efficiency distraction indices, separately for each valence. Negative distraction was reduced in the reward group $(M=6 \mathrm{~ms}, S D=39 \mathrm{~ms})$ relative to the control group $(M=29$ $\mathrm{ms}, S D=50 \mathrm{~ms}), t(88.7)=2.58, p=.012, d_{s}=0.526,95 \% \mathrm{CI}[5,42]$; likewise, positive distraction was reduced in the reward group $(M=19 \mathrm{~ms}, S D=52 \mathrm{~ms})$ relative to the control 
Motivation and Emotional Distraction

group $(M=50 \mathrm{~ms}, S D=74 \mathrm{~ms}), t(84.7)=2.41, p=.018, d_{s}=0.491,95 \% \mathrm{CI}[5,57]$; in contrast, neutral distraction did not differ between the reward $(M=11 \mathrm{~ms}, S D=36 \mathrm{~ms})$ and control $(M=10 \mathrm{~ms}, S D=38 \mathrm{~ms})$ groups, $t(93.5)=0.09, p=.930, d_{s}=0.018,95 \%$ CI $[-14$, 16]. Thus, when taking accuracy into account, reward reduces emotional distraction, but not neutral distraction. Importantly, the fact that the reward group $\times$ distractor presence interaction, and the reward group $\times$ distractor presence $\times$ distractor valence interaction, remained significant when taking accuracy into account demonstrates that a strategy of sacrificing accuracy for speed does not account for the effect of reward on emotional distraction.

\section{Comparing the influence of monetary versus non-monetary reward}

To address the question of whether monetary and non-monetary reward differently influenced distraction, we combined data from Experiment 3 and 4 and conducted an exploratory ANOVA on distraction indices [distractor-present RT - distractor-absent RT], with experiment (monetary reward, non-monetary reward) and reward group (control, reward) as between-subjects factors, and distractor valence (negative, neutral, positive) as the withinsubjects factor. Importantly, there was no significant main effect of experiment, $F(1,156)=$ $0.01, p=.925, \eta_{p}{ }^{2}<.001, \omega^{2}<.001$, indicating that monetary and non-monetary reward did not differ in their overall influence on distraction.

Main effects of reward group, $F(1,156)=19.38, p<.001, \eta_{p}{ }^{2}=.111, \omega^{2}=.104$, and distractor valence, $F(2,312)=9.60, p<.001, \eta_{p}{ }^{2}=.058, \omega^{2}=.050$, were qualified by the significant experiment $\times$ reward group $\times$ distractor valence interaction, $F(2,312)=3.28, p=$ $.039, \eta_{p}{ }^{2}=.021, \omega^{2}=.013$. This reflected the fact that, in Experiment 3, monetary reward significantly reduced distraction overall (with no interaction with distractor valence), whereas in Experiment 4, non-monetary reward significantly reduced positive and negative, but not neutral distraction (see both above Results sections for follow-up tests, separately for each experiment; and for further discussion of these findings see Chapter 5: General Discussion).

In summary, in Experiment 4, distraction was greater for emotional compared to neutral images, and symbolic performance-contingent non-monetary reward reduced distraction by peripherally-presented task-irrelevant emotional images. Findings suggest that motivation, elicited by non-monetary reward, can enhance effective control of attentional capture by emotional distractors. 


\section{General Discussion}

As expected, consistent with Experiments 1 and 2, high arousal positive images of erotic couples and negative images of mutilations were more distracting than neutral images of people. In two experiments, we showed that monetary reward (Experiment 3), and nonmonetary reward (Experiment 4), reduced emotional distraction from positive and negative images. These findings extend those of Experiments 1 and 2 - in which reward reduced distraction from images that appeared centrally, within the spatial locus of attention, by showing that reward reduced distraction from images appearing in peripheral locations, outside the spatial locus of attention. Findings from this chapter therefore extend the small but growing literature that reports a beneficial influence of motivation on control of distraction by centrally-presented negative emotional images (Padmala \& Pessoa, 2014; Padmala et al., 2017); current findings suggest that motivation may also enhance control of attentional capture by peripheral emotional distractors (but see below and Chapter 5: General Discussion for a more detailed discussion).

The current study also extends findings on the benefit of motivation on cognitive control to include the influence of non-monetary reward. Consistent with our finding that monetary and non-monetary reward both attenuated emotional distraction, Shen and Chun (2011) found that both symbolic and monetary reward facilitated task performance. We found that monetary reward was no more effective than non-monetary reward at reducing distraction. This finding is contrary to that of Hübner \& Schlösser (2010) and Dambacher et al. (2011), who found that monetary reward enhanced flanker task performance to a greater degree than non-monetary reward when payoff conditions emphasised speed over accuracy (as did ours). Several differences between ours and Schlösser and colleagues' studies could account for our contrary findings. First, their task was more difficult than ours, as shown by their lower levels of accuracy in the flanker task. Second, their task was emotionally-neutral. Third, our reward manipulations included affectively positive performance feedback whereas theirs included affectively neutral performance feedback. Future work could compare these factors to determine their influence on the effect of monetary versus non-monetary reward on cognitive control.

One interesting broader theoretical question is whether motivational effects on cognitive control are general, or specific to the type of reward provided. Note that both monetary reward and non-monetary reward (e.g., points) are secondary reinforcers that have gained their value via associative learning. In our lives we experience various primary 


\section{Motivation and Emotional Distraction}

reinforcers (e.g., food, sex or drugs), and secondary reinforcers (e.g., money, points, or praise). Although delivery of performance-contingent sweet liquid (a primary reinforcer) can enhance cognitive control (Beck, Locke, Savine, Jimura, \& Braver, 2010; Chiew \& Braver, 2016), evidence is mixed as to whether different primary and secondary reinforcers are coded and evaluated on a common scale in reward processing regions (Beck et al., 2010; Bickel, Pitcock, Yi, \& Angtuaco, 2009; Bray, Shimojo, \& O’Doherty, 2010; Krug \& Braver, 2014; Miyapuram, Tobler, Gregorios-Pippas, \& Schultz, 2012). Future work will benefit from comparing the separate and combined influence of different types of primary and secondary reinforcers on cognitive control.

Beyond theoretical concerns, the fact that cognitive control is influenced by reward manipulations (even non-monetary ones) has broader implications for all psychological empirical research. Different research groups tend to use different types of performance feedback and encouragement (often not as a manipulation, but as part of the task) and these may only be reported casually in method sections, if at all (Ravizza \& Delgado, 2014; Ravizza, Goudreau, Delgado, \& Ruiz, 2012); our findings show that it is important for researchers to consider the potential influence of including performance feedback when designing experiments, and to report them in method sections. On another practical level, not having to pay participants money to elicit motivation has obvious value when designing future reward experiments. Our finding also has implications for how motivation can influence our performance outside of the lab (e.g., in the workplace, playing sports, driving, etc.). Monetary reward is not always practical or possible, and so investigating how different forms of reward and feedback can influence motivation (and in turn, cognitive control) could prove fruitful.

A major consideration is that in Experiment 3, participants were recruited from the community and were paid for their time; in Experiment 4, participants were recruited from the introductory psychology pool and were compensated with course credit. These different reasons for participating in the two experiments might influence intrinsic task motivation, and thus cognitive control (Bowen \& Kensinger, 2017; Krug \& Braver, 2014). Participants from the community could have participated because they wanted the money, out of genuine interest, out of altruism, or any number of factors. In contrast, psychology student participants were more likely to have participated out of an obligation to gain course credit. However, contrary to the notion that student samples might lack motivation (Krug \& Braver, 2014), participants in the control conditions of the two experiments (which were methodologically identical) showed similar levels of distraction overall. In Experiment 3, 


\section{Motivation and Emotional Distraction}

control participants averaged $29 \mathrm{~ms}(S D=26 \mathrm{~ms})$ of distraction (collapsed across valence), and in Experiment 4 control participants averaged $30 \mathrm{~ms}(S D=36 \mathrm{~ms})$ of distraction, suggesting that their potential different motivations for completing the study did not influence baseline levels of cognitive control or distraction.

Non-monetary reward reduced emotional but not neutral distraction. In contrast, monetary reward reduced both emotional and neutral distraction. However, I think it would be premature to draw conclusions about the influence of symbolic versus monetary reward based on this difference, especially given that in Experiments 1 and 2, monetary reward reduced emotional but not neutral distraction. Neutral distraction in the control group of the monetary reward experiment was higher $(24 \mathrm{~ms})$ than in the non-monetary reward experiment (13 ms). Thus, neutral distraction could have had more "room to move" in the monetary reward experiment; and may have been closer to floor in the control group in the nonmonetary reward experiment. It is possible that the monetary reward experiment (with $n=32$ per group) lacked power to detect a three-way reward group $\times$ distractor presence $\times$ distractor valence interaction. The three-way interaction found in Experiment 4 is consistent with the hypothesis that reward (over and above upregulating control) might enhance the motivational value of the target stimuli, allowing them to better compete for attention with the emotional distractors. Because neutral images have less motivational value, neutral distraction would not benefit as much from this altered motivational competition. I return to this point in Chapter 5: General Discussion. Note that we found no evidence for the alternative hypothesis, that reward might be less effective at reducing emotional versus neutral distraction because of our strong attentional biases to emotional content.

Here, we cannot determine the mechanisms by which reward reduced distraction from peripherally-presented images (see Chapter 5: General Discussion for further discussion of potential differences between mechanisms of control of central versus peripheral distractors). It is possible that reward influenced proactive, location-based, suppression mechanisms to help prevent attentional capture by emotional distractors (e.g., Murphy et al., 2018). However, reactive mechanisms might also play a role, especially when distractors are emotional. If something has emotional value, perhaps we always select it for our attention to determine whether it needs immediate action, and then rapidly disengage from it if not (as is also sometimes found with salient singleton distractors; Belopolsky, Schreij, \& Theeuwes, 2010; Theeuwes, de Vries, \& Godijn, 2003). It is also possible, as suggested by pupil dilation findings from Experiment 2, that reward might engage proactive mechanisms (e.g., by enhancing goal representation prior to distractor onset), which then facilitate rapid reactive 
Motivation and Emotional Distraction

control processes post-distractor. But this strategy of preparing to rapidly disengage might be particularly beneficial when distractors are presented centrally, within the spatial locus of attention. In the current study, we cannot disentangle whether reward enhanced proactive control suppression mechanisms to help prevent attentional capture, reactive control mechanisms to facilitate disengagement, or some combination of both. Future work using time-sensitive electrophysiological and psychophysiological measures (such as pupillometry, as was used in Chapter 3) will help to elucidate the timecourse of motivation-elicited shifts in control of peripheral emotional distractions. 
Motivation and Emotional Distraction

\section{Chapter 5: General Discussion}

The aim of this thesis was to determine whether, and how, motivation enhances attentional control of positive and negative emotional distractions. In four experiments I addressed this question by having participants complete a simple visual task while attempting to ignore emotional and neutral distractor images. To manipulate task motivation, half the participants completed the task in the absence of performance-contingent reward; the other half had the opportunity to earn reward for fast and accurate performance. Distraction was indexed by slowed performance on distractor trials, relative to a scrambled distractor baseline or a distractor-absent baseline. As expected, positive and negative images were more distracting than neutral ones. In all four experiments, reward reduced, but did not eliminate, distraction from positive and negative emotional images. Findings therefore extend the beneficial influence of task motivation on attentional control to negative and positive emotional distractions.

In Experiment 1 (Chapter 2) I found that reward reduced emotional distraction from task-irrelevant images that appeared within the spatial locus of attention. Experiment 2 (Chapter 3) replicated that behavioural finding and tracked pupil dilation over time to shed light on the timecourse by which reward enhances control of emotional distractions. Pupil dilation findings were consistent with reward-elicited upregulation of dynamic proactive control mechanisms prior to stimulus-onset, that then facilitated rapid implementation of reactive control mechanisms post-distractor. In Experiments 3 and 4 (Chapter 4), the distractors appeared peripherally, outside the spatial locus of attention. In this context, the distractor must capture attention away from the central letter targets to impair performance. In both Experiments 3 and 4, reward reduced attentional capture by emotional distractors. In Experiment 4, the points did not represent greater monetary reward. Remarkably, this purely non-monetary reward reduced distraction to a similar extent to the monetary reward manipulation in Experiment 3.

My thesis addressed several key questions that I will discuss in turn. First, does motivation enhance control of emotional and neutral distractions to different degrees? Second, does motivation enhance control of emotional distractions via a shift to proactive control? Third, does motivation enhance control of emotional distractors when they appear outside the current spatial locus of attention, as well as within the current spatial locus of attention? And finally, does motivation enhance control of positive and negative distractions to different degrees? Ideas for future work to address further theoretical issues will be 
Motivation and Emotional Distraction

discussed in relation to these questions. I then discuss some thoughts on reward; potential real world applications; and finally, I discuss limitations, and future directions to address those limitations.

\section{Does motivation enhance control of emotional and neutral distractions to different degrees?}

Motivation is thought to enhance attentional control of emotionally-neutral distractions by encouraging a shift to more effective proactive control (Botvinick \& Braver, 2015; Yee \& Braver, 2018). My thesis addressed the question of whether motivation can also enhance cognitive control of emotional distractions, which are more difficult to ignore. It is therefore useful to compare the influence of performance-contingent reward on emotional distraction, to its influence on neutral distraction. Only two previous studies have shown the beneficial influence of reward on distraction from negative emotional images (Padmala \& Pessoa, 2014; Padmala et al., 2017). However, Padmala and colleagues' experimental design used trials on which a neutral distractor image appeared as a baseline from which to measure slowing on negative distractor trials. Therefore, they had no index of neutral distraction to compare to their index of negative distraction. In contrast, my experiments used scrambled distractor, or distractor-absent, baseline conditions. This allowed me to index negative, positive, and neutral distraction to test hypotheses regarding how control of emotional and neutral distraction might differ.

I had two hypotheses regarding how motivation might influence emotional distraction differently to neutral distraction, which led to opposing predictions. First, I reasoned that, given their importance for survival and reproduction (LeDoux, 2012; Rolls, 2000), attention to emotional distractors may be less susceptible to manipulations of control than attention to neutral distractors. In this case, reward would reduce neutral distraction to a greater extent than emotional distraction. There are many reasons to think that emotional distractions may be more difficult to control than neutral ones. Our attention is heavily biased to emotional relative to neutral stimuli, via endogenous, exogenous, and selection history mechanisms that guide attentional selection (Awh et al., 2012; Belopolsky, 2015; Brosch et al., 2011; Carretié, 2014; Mohanty \& Sussman, 2013; Yiend, 2010). Biases to emotional content may also occur via an emotion-specific attentional mechanism (Pourtois et al., 2013; Vuilleumier, 2015). Furthermore, attention to highly arousing emotional content consumes some of the same common-pool cognitive resources that are recruited for cognitive control (Pessoa, 2009). Thus, if some of the same cognitive resources needed to control distraction are occupied by processing the emotional distractors, it seems reasonable that emotional distraction may be 


\section{Motivation and Emotional Distraction}

more difficult to control than neutral distraction. It has even been claimed that processing of emotional distractors occurs automatically, without intention, at least when sufficient attentional resources are available (Okon-Singer et al., 2007; Pessoa, 2005). The task I used in all experiments was low in perceptual load (i.e., it is perceptually simple to identify a $\mathrm{K}$ or $\mathrm{N}$ amongst an array of O's; Lavie et al., 2004) so that attentional resources would be available to process the distractors. If emotional distraction occurs without intention in these circumstances, then it should not be reduced by manipulations that increase cognitive control. However, I found no evidence to support the hypothesis that neutral distraction would be reduced to a greater extent than emotional distraction. In contrast, in three out of four experiments (all but Experiment 3; discussed below) reward reduced emotional distraction to a greater extent than neutral distraction. Thus, not only does motivation enhance control of emotional distraction, it does so at least as effectively as it does for neutral distraction.

This finding, that motivation enhances control of emotional distraction to a greater extent than neutral distraction, supports the alternative hypothesis; that reward might alter the outcome of goal-driven attentional competition between targets and distractors. Attentional biases to emotional stimuli are partly driven by endogenous mechanisms (e.g., Mohanty \& Sussman, 2013); they are relevant to our fundamental, biologically-determined goals of surviving and reproducing (LeDoux, 2012; Rolls, 2000). The letter targets are also goalrelevant; participants must attend to them to successfully complete the task. The letters and distractors therefore compete for our endogenous, goal-driven attention. The neutral images also have biological-relevance, because they are scenes of people, but less so than high arousal erotic or gruesome distractor images. We are therefore more motivated to attend to emotional than neutral images. Rewarding people for attending to the letter targets might enhance the letters' motivational value, increasing their goal-relevance, thereby altering the outcome of the goal-driven competition between the letters and distractors. The letters could gain reward value by associative learning, as they are repeatedly paired with reward feedback on successful reward trials. The letters could also gain reward value by instrumental conditioning; responding to them quickly and accurately results in reward feedback. Because goal-driven attentional mechanisms drive attention to emotional images to a greater extent than neutral ones, enhancing the motivational value of the letter targets should influence goaldriven attentional competition between the targets and emotional stimuli to a greater extent than targets and neutral stimuli. Supporting this hypothesis, in three out of four experiments I found a significant three-way reward $\times$ distractor type $\times$ distractor valence interaction; reward reduced emotional to a greater extent than neutral distraction. The lack of a three-way 


\section{Motivation and Emotional Distraction}

interaction in Experiment 3 could be due to insufficient statistical power; that study had the smallest sample size $(n=64)$ of the four experiments ${ }^{6}$.

From previous findings, we know that reward enhances proactive control in emotionally-neutral contexts, thereby reducing distraction (Botvinick \& Braver, 2015; Yee \& braver, 2018). Current findings extend this by showing that reward also enhances control of emotional distractions, which are harder to ignore. But, as discussed above, in addition to this influence of motivation on cognitive control, reward may reduce distraction by influencing the outcome of goal-driven attentional competition between letter targets and distractors. Note that this mechanism is likely to be specific to reward as a control manipulation, and presumably would not occur with other manipulations of control. For example, increasing distractor frequency should not alter the goal-driven competition between targets and distractors. Like reward, however, in three experiments, increasing the frequency of peripherally-presented distractors also reduced emotional to a greater extent than neutral distraction (Grimshaw et al., 2018; Murphy et al., 2018). But, in those distractor frequency experiments, neutral distraction was eliminated in the high frequency condition $(-2 \mathrm{~ms} ; 1 \mathrm{~ms}$; 7 ms of neutral distraction, all not significantly different from 0 ). Thus, although emotional distraction was reduced to a greater degree than neutral distraction, neutral distraction could not have been reduced more. Therefore, the distractor frequency $\times$ valence interaction could reflect a floor effect of distractor frequency on neutral distraction, rather than reflecting a difference in the mechanisms by which distractor frequency influences emotional, versus neutral, distraction. In contrast, the reward condition of my two peripheral distractor experiments (Chapter 4) showed $7 \mathrm{~ms}$ (not significantly different from 0 ), and $9 \mathrm{~ms}$ (significantly different from 0), of distraction, respectively. Thus, in Experiment 4, neutral distraction could have been further reduced, suggesting that a floor effect may not account for my finding that reward reduced emotional to a greater degree than neutral distraction. However, I cannot completely rule out a floor effect on this basis. Future work directly comparing the influence of reward to other manipulations of control, as well as manipulating the motivational value of the distractors (e.g., smoking related stimuli in smokers in varying degrees of deprivation) will be useful for determining the extent to which reward specifically alters goal-driven competition between targets and distractors.

\footnotetext{
${ }^{6}$ This was because Experiment 3 was actually run first in this sequence of studies, and we did not know how large to expect the influence of reward on emotional distraction to be in a peripheral distractor task at that point. Subsequent experiments were higher powered (Experiment 1: $n=72$; Experiment 2: $n=110$; Experiment 4: $n=$ 96).
} 


\section{Motivation and Emotional Distraction}

Across the four experiments, the influence of reward on neutral distraction was inconsistent. In Experiments 1 and 4, reward did not significantly reduce neutral distraction. In contrast, in Experiment 2 and 3, reward did significantly reduce neutral distraction. This inconsistency is somewhat surprising given the decade of literature on the beneficial influence of motivation on resolving conflict, and controlling attention, in emotionallyneutral contexts (Botvinick \& Braver, 2015; Yee \& Braver, 2018). The pattern of findings cannot be accounted for by systematic experimental parameters like distractor location (central versus peripheral) or type of reward (monetary versus non-monetary). For example, across experiments, reward significantly reduced neutral distraction under both central (Experiment 2) and peripheral presentation conditions (Experiment 3); but also did not reduce distraction under central (Experiment 1) and peripheral presentation conditions (Experiment 4). However, it is notable that, in all experiments, neutral distraction was numerically lower in the reward group than the control group. Thus, it seems more likely that, rather than systematic experimental factors influencing whether neutral distraction was reduced by reward, the effect of reward on neutral distraction is small (relative to the effect of reward on emotional distraction) and is therefore not always observed.

To determine whether reward reduces emotional distraction differently to neutral distraction, I can also turn to the influence of reward on post-stimulus pupil dilation. In Experiment 2 I measured post-stimulus pupil dilation following intact relative to scrambled distractors, to index changes in distractor processing due to reward. Post-stimulus pupil findings were largely consistent with behavioural distraction findings: emotional distractors produced greater pupil dilation than neutral ones; and dilation to intact (relative to scrambled) distractors was reduced by reward. Moreover, greater pupil dilation in response to emotional distractors predicted greater behavioural emotional distraction, demonstrating the validity of the pupil response as a measure of emotional processing. Unlike the behavioural findings, however, the interaction between reward and valence was not observed in post-stimulus pupil dilation. Rather, reward reduced post-distractor pupil dilation, but the reduction was not dependent on the emotional value of the distractors. Numerically - but not significantly reward did reduce the pupil response to emotional distractors to a greater extent than neutral ones (see Table 3.3). Thus, this lack of a significant reward $\times$ valence interaction in the poststimulus pupil index could reflect a lack of power to detect this effect. Pupillometry experiments that manipulate reward within-subjects may have better power to determine whether reward reduces dilation to emotional distractors to a greater extent than neutral ones. In summary, findings show that reward reduces emotional distraction at least as effectively as 
Motivation and Emotional Distraction

it does neutral distraction. The section below discusses the possible mechanisms by which this might occur.

\section{Does motivation enhance control of emotional distractions via a shift to proactive control?}

The DMC framework (Braver, 2012; Braver et al., 2007) posits that we flexibly shift between using reactive control mechanisms, implemented post-distractor, to using proactive control mechanisms, implemented pre-distractor, depending on the context. Reactive control is our default strategy, because it requires less cognitive effort. We switch to a proactive control strategy when the benefits of exerting the extra mental effort outweigh the metabolic costs (Kool et al., 2017; Shenhav et al., 2013). For example, when conflict is experienced frequently (Bugg \& Crump, 2012) or distractors are frequently presented (Forster \& Lavie, 2008; Grimshaw et al., 2018), we switch to a proactive control strategy to more effectively resolve conflict or to control distraction. When task motivation is increased by availability of performance-contingent reward, it becomes beneficial to switch to a proactive control strategy to ignore distractions and so maximise reward (Botvinick \& Braver, 2015; Yee \& Braver, 2018). The vast majority of studies that have tested predictions from the DMC framework have used emotionally-neutral contexts (e.g., Chiew \& Braver, 2013, 2014; Padmala \& Pessoa, 2011). Findings from Padmala and colleagues (Padmala \& Pessoa, 2014; Padmala et al., 2017) show that we may be able to extend the influence of motivation on proactive control within the DMC framework to include control of negative distractions. My findings extend this conclusion further to include control of positive distractions.

It is well-established that reward enhances proactive control in non-emotional contexts (Botvinick \& Braver, 2015; Yee \& Braver, 2018). But reward can also upregulate reactive control to resolve response and stimulus conflict (e.g., Boehler et al., 2014; Hopf et al., 2015; Krebs et al., 2010). There is mixed evidence in the literature as to whether attentional suppression of neutral distractors is different to suppression of emotional distractors. For example, recent findings indicate that the $\mathrm{P}_{\mathrm{d}}$ (an ERP index of active suppression; Gaspelin \& Luck, 2018) is delayed for spider distractors, relative to leaf distractors (Burra et al., 2018), indicating that there may be differences in the timing of suppression of attentional capture by emotional versus neutral distractors. But a study from our lab (Murphy et al., 2018) found no difference in tonic alpha suppression - an index of sustained proactive attentional control - when distractors were expected to be emotional, versus when they were expected to be neutral. Given the evolutionary importance of attending to emotional stimuli (LeDoux, 2012; Rolls 2000), I reasoned that we may always 


\section{Motivation and Emotional Distraction}

attend to emotional stimuli, regardless of their relevance to task goals, and then use reactive mechanisms to suppress distractors and/or disengage and shift attention back to the targets if they do not require further attention or action. In contrast, because neutral stimuli are less important for survival and reproduction, it may be possible to use proactive mechanisms to entirely prevent attentional capture by neutral distractions. Alternatively, I reasoned that reward may elicit greater proactive effort to control emotional than neutral distractors, because they are harder to ignore. Experiment 2 was specifically designed to track the timecourse of reward's influence on control of emotional versus neutral distractions. I did this by tracking pupil dilation as an index of mental effort over time (van der Wel \& van Steenbergen, 2018).

I examined two types of proactive control by examining two different indices of pupil dilation. The first was sustained proactive control, a stable adjustment to control across entire blocks of trials, which was indexed by tonic dilation. Tonic dilation was measured during the $1000 \mathrm{~ms}$ inter-trial-interval, baselined to a $200 \mathrm{~ms}$ inter-trial-interval period during the baseline block (which included only scrambled distractors, and no reward). Tonic dilation could reflect upregulation of goal representations (i.e., attend to the letters, and ignore the distractors), or stable changes to neural competition in visual cortex to highlight target locations and/or suppress distractor locations, across blocks of trials (e.g., Murphy et al., 2018). The tonic pupil index did not significantly differ between the reward and control groups, suggesting that motivation did not influence sustained proactive control. However, tonic pupil dilation was significantly greater in blocks with emotional distractors relative to blocks with neutral distractors. Furthermore, the tonic proactive control index on emotional blocks significantly and negatively correlated with behavioural measures of emotional distraction; higher tonic dilation was associated with less emotional distraction. This finding suggests that this increase in sustained pupil dilation during emotional blocks reflects greater sustained control (rather than emotional arousal due to the distractors on previous trials). Greater sustained emotional arousal would predict the opposite effect; a positive correlation between tonic dilation and behavioural emotional distraction. Thus, I found evidence for greater sustained proactive control of emotional relative to neutral distractors, but this sustained control was not modulated by reward.

The second index of proactive control was dynamic proactive control, a transient upregulation of control within a trial, which was indexed by phasic pupil dilation during the $2000 \mathrm{~ms}$ period prior to stimulus-onset (baselined to an immediately prior time-period). Phasic pupil dilation indexes dynamic changes to control in anticipation of conflict or 


\section{Motivation and Emotional Distraction}

distraction. Interestingly, the phasic proactive control pupil index was not modulated by distractor valence (which was predictable within a block). Thus, I found no evidence that dynamic implementation of proactive control was influenced by the emotional value of an expected distractor. But exploratory analyses - which tracked differences in pupil dilation over time - showed that reward facilitated dynamic implementation of proactive control before stimulus-onset, regardless of the emotional valence of the expected distractor. This could reflect any number of proactive control mechanisms, including preparation to attend to the target features or locations, or to suppress distractor content, or distractor location.

I note that the pupil response has a time-lag. Emotional arousal modulates pupil dilation with a delay of around $500 \mathrm{~ms}$, but it is not known how long the delay is for the influence of cognitive effort on dilation; although single-cell recordings in monkeys show that pupil dilation peaks around $300 \mathrm{~ms}$ post-activation of LC (Joshi et al., 2016). This means that I cannot know precisely when, within the pre-stimulus period, reward started to influence control processes. However, because reward influences pupil dilation before stimulus-onset at all, I can be confident that the dynamic increase in pupil dilation occurs in anticipation of distraction does indeed reflect proactive control. ERP and EEG studies, that have finer temporal resolution than pupillometry, will be necessary to better elucidate the specific timing of proactive control of emotional distraction. In summary, dynamic proactive control was modulated by availability of reward, but not by the expected emotional valence of the distractors. In contrast, sustained proactive control was modulated by the expected emotional valence of the distractors, but not by availability of reward.

According to the DMC framework, enhancing proactive control reduces the need for reactive control, because doing so reduces conflict or distraction before it occurs (Braver, 2012; Braver et al., 2007). However, it is also possible that, under some conditions, proactive and reactive control could work in tandem (e.g., Schevernels et al., 2015). For example, one could proactively prepare - e.g., by holding the goal in working memory - to rapidly disengage from distractors. In line with this notion, in Experiment 2, the increased phasic pupil dilation under reward began prior to stimulus-onset and continued well after distractorpresentation ( $2400 \mathrm{~ms}$ post-stimulus onset). Even allowing for a time-lag on the pupil response, this finding clearly suggests upregulation of reactive mechanisms under reward. This pattern suggests that reward enhanced dynamic proactive control prior to distractoronset, which then facilitated rapid implementation of reactive control mechanisms to successfully disengage from distractors. 
Motivation and Emotional Distraction

This observed pattern in the pupil data in Experiment 2 - indicating dynamic proactive preparation to rapidly disengage from central distractors -is consistent with Theeuwes' rapid disengagement hypothesis. Theeuwes, Atchley \& Kramer (2000; see also Theeuwes, 2003, 2010) proposed that on viewing a scene, attention is reflexively directed to the most salient item in the scene. Only then do endogenous mechanisms guide attention based on current goals. In my task, this would predict initial attentional selection of distractors, especially emotional distractors, as they are more salient than the letter targets. Attention would then be rapidly disengaged from the goal-irrelevant distractor in order to perform the task. In line with Theeuwes' rapid disengagement hypothesis, there is evidence that endogenous settings cannot override exogenous attentional capture by goal-irrelevant salient distractors (Belopolsky et al., 2010). Rather, after initial attentional capture by a salient distractor, attention is rapidly disengaged and shifted according to current goals (Belopolsky et al., 2010; Geng, 2014). But not all previous findings are consistent with Theeuwes' rapid disengagement hypothesis. For example, Folk et al. (1992) proposed that initial attentional capture on viewing a scene is contingent on current endogenous attentional settings, and is therefore not purely exogenous. Also challenging Theeuwes' rapid disengagement hypothesis, Luck and colleagues (Gaspelin, Leonard, \& Luck, 2015; Gaspelin \& Luck, 2018; Sawaki \& Luck, 2010) proposed that salient distractors elicit an "attend to me" signal that can be actively suppressed in anticipation of stimulus-onset, proactively preventing attentional capture. In summary, there is little agreement regarding whether we can entirely prevent attention to non-emotional salient distractors, or whether we select them for attention before rapidly disengaging from them. Given these controversies, it is premature to draw conclusions regarding the specific mechanisms that might be involved in attentional selection and control of emotional distractions.

Future electrophysiological studies will be valuable for elucidating the specific proactive and reactive mechanisms that control emotional versus non-emotional distraction. For example, ERP and EEG studies examining anticipatory components that index proactive control (e.g., the contingent negative variation; van den Berg et al., 2014; and alpha suppression; Murphy et al., 2018); components that reflect active suppression (e.g., the $\mathrm{P}_{\mathrm{d}}$; Gaspelin \& Luck, 2018); and post-stimulus components that index emotional processing (e.g., the EPN and LPP; Schupp et al., 2006) will help to more precisely elucidate the timing and nature of proactive and reactive mechanisms induced by reward. Additionally, does reward suppress tonic anticipatory alpha oscillations - thought to reflect enhanced sustained proactive control of emotional distractions - as distractor frequency did in one study from our 
Motivation and Emotional Distraction

lab (Murphy et al., 2018)? Or does reward suppress phasic alpha - reflecting enhanced dynamic proactive control - in line with the pupil findings here? One consideration is that Murphy and colleagues used a peripheral distractor paradigm in their EEG study of distractor frequency, whereas I used a central distractor paradigm in my pupillometry study. Future ERP and EEG work that directly compares the influence of different control manipulations, on central and peripheral distraction, will be useful. Different mechanisms may be used to control emotional distraction under different control manipulations, and under different distractor presentation conditions.

\section{Does motivation enhance control of emotional distractors when they appear outside the current spatial locus of attention, as well as within the current spatial locus of attention?}

In Experiments 1 and 2, the distractors appeared centrally, within the current spatial locus of attention ${ }^{7}$ - we instruct participants to look at the central fixation cross at the start of each trial. In contrast, in Experiments 3 and 4, the distractors appeared peripherally, outside the current spatial locus of attention. Different mechanisms of control might be available under these different presentation conditions. Active distractor suppression can occur by suppressing distractor features (e.g., colour), or distractor location (Gaspelin \& Luck, 2018). Control of central versus peripheral distractions could plausibly occur via similar mechanisms that suppress distractor content; but might occur via different mechanisms that suppress location. Location has special status in attentional selection. Neural competition in visual cortex is mapped according to retinal location, with mechanisms of attentional selection enhancing the gain of some locations, and suppressing the gain of others (Desimone \& Duncan, 1995; Kastner \& Ungerleider, 2001). Processing at locations at which salient singleton distractors are expected to occur can be suppressed to below baseline levels (Gaspelin \& Luck, 2018), showing that peripheral locations can be successfully suppressed. But it seems plausible that proactive, location-based, mechanisms of attentional suppression may not work as effectively for stimuli that appear within the current spatial locus of attention. In line with this notion, Beck and Lavie (2005) found that distraction was greater when distractors appeared centrally, than when they appeared peripherally. Thus, the observed strategy (in Experiment 2), of proactively preparing to rapidly disengage attention,

\footnotetext{
${ }^{7}$ I note that across all experiments, although I direct people to look at the central fixation cross, their attention could be covertly directed elsewhere (Posner, 1980). For example, people can covertly direct their attention into a donut shape (Müller \& Hübner, 2002) despite their eyes being directed at the centre of the screen. In the central distractor task, participants could covertly attend to the letter target locations, despite their eyes being directed to the centre of the screen. However, I observe such large distraction effects in the central distractor task, and distraction was not eliminated under reward, so it seems likely that participants were indeed directing their attention to the centre of the screen in the tasks.
} 


\section{Motivation and Emotional Distraction}

may be particularly effective under conditions in which distractors appear centrally. In contrast, when distractors appear peripherally, additional, location-based, suppression mechanisms may be available to proactively prevent attentional capture. But, to further complicate matters, Chen \& Treisman (2008) found that, under certain conditions, distractors can actually be controlled more effectively when they appear centrally, compared to when they appear peripherally. Thus, it is not clear whether anticipatory, location-based suppression can prevent processing of stimuli that appear centrally, within the spatial locus of attention. It is also unclear whether attention to emotional distractors can be entirely suppressed, regardless of whether they appear centrally or peripherally.

In summary, there is little agreement regarding the specific mechanisms that cause and control - distraction from central versus peripheral stimuli, even in non-emotional contexts. Future work that systematically manipulates the predictability of distractor content, and distractor location, could help to determine which specific proactive mechanisms are facilitated by reward, in non-emotional, and emotional, contexts. For example, if distractor location (but not distractor content) is predictable, location-based proactive mechanisms should be available to control distraction. In contrast, if distractor content (but not distractor location) is predictable, proactive mechanisms that suppress distractor content (perceptual or semantic) should be available to control distraction. There is much still to learn about the specific mechanisms by which distraction is controlled.

\section{Does motivation enhance control of positive and negative distractions to different} degrees?

Consistent with other studies that matched positive and negative distractors on subjective arousal ratings (e.g., Grimshaw et al., 2018; Gupta et al., 2016; Most et al., 2007; Padmala et al., 2018), here, negative images of mutilations, and positive images of erotic couples, were similarly distracting. Furthermore, both negative and positive images were consistently more distracting than neutral images of people in everyday scenarios, consistent with the notion that distractor arousal, rather than distractor valence, influences the magnitude of distraction (Mather \& Sutherland, 2011; Padmala et al., 2018; Pool et al., 2016; Vogt et al., 2008). Moreover, the fact that reward attenuated both positive and negative to a greater extent than neutral distraction suggests that the impact of motivation on control is dependent on distractor arousal, rather than distractor valence. Furthermore, pupil findings suggest that the timecourse of the influence of reward on positive and negative distraction did not differ. However, just because control of positive and negative distractions occurred via a 


\section{Motivation and Emotional Distraction}

similar timecourse, it does not mean they are controlled by the same specific attentional mechanisms.

Two previous studies showed the benefit of reward on distraction from negative images (Padmala \& Pessoa, 2014; Padmala et al., 2017). My findings show that reward reduces negative and positive distraction to similar degrees. This finding is consistent with Grimshaw and colleagues' (2018) finding that increasing distractor frequency (a manipulation of proactive control) also reduced both positive and negative distraction. My finding is also somewhat consistent with Kennedy and colleagues' (2017) finding that warning participants about the content of upcoming emotional distractors (e.g., "ignore gruesome", or "ignore erotic") slightly reduced both positive and negative distraction. However, their task was very different to the task used here, with their task indexing emotional distraction over time; emotional distractors were presented shortly before a nonemotional target in a rapid stream of images. Furthermore, a recent (not yet published) collaborative replication of Kennedy and colleagues' study (Walsh, Zhao, Grimshaw, \& Most, in prep.) did not find a benefit of these warnings for either positive or negative distraction.

But my finding, that reward reduced both positive and negative distraction, contrasts other studies' findings that non-reward manipulations of control reduce negative but not positive distraction. For example, Most and colleagues (2007) presented emotional distractors shortly before non-emotional target images in a rapid image stream. When they told participants what type of non-emotional target to look out for, negative - but not positive distraction was reduced. Furthermore, Gupta and colleagues (2016) used a similar letter identification task to the one used here, with centrally-presented positive and negative distractors. They found that increasing the task's perceptual load (by including other letters that were more perceptually similar to K's and N's, instead of O's) reduced negative but not positive distraction. Thus, sometimes, consistent with my findings, manipulations of control reduce both positive and negative distraction (Grimshaw et al., 2018; Kennedy et al., 2017); and sometimes they influence negative but not positive distraction (Gupta et al., 2016; Most et al., 2007).

Although we are biased to attend to both positive and negative stimuli, the function of attending to positive and negative stimuli is to elicit approach and withdrawal motivation, respectively. Given these opposing motivational directions, the mechanisms underlying biases to positive and negative stimuli, and the factors that influence control of these biases, could also differ. For example, positive stimuli signal potential reward, which motivates us to 


\section{Motivation and Emotional Distraction}

approach; in contrast, negative stimuli signal potential threat, which motivates us to withdraw. If attention is not quickly directed to a positive stimulus, we may miss out on a reward, but this most likely will not be an immediate threat to our survival. But if we do not quickly attend to a potential threat, this could present an immediate danger. It has therefore been proposed that attention is selectively and automatically biased toward threatening stimuli via exogenous mechanisms (e.g., Mulckhuyse, 2018; Öhman et al., 2001). However, more recent evidence suggests that arousal, and relevance to the individual at that moment, determines the magnitude of attentional biases to emotional stimuli, rather than their valence (e.g., Pool et al., 2016).

Another consideration is that attention to positive distractors may be more strongly driven by endogenous mechanisms than attention to negative distractors. We want to attend to positive distractors more than we do negative ones, because positive stimuli are rewarding. Given the choice, most people would prefer to look at erotic than gory stimuli. Thus, because various manipulations of control may influence goal-driven attention to the targets or distractors to different degrees, they might have different effects on positive and negative distraction. Because reward is a manipulation that increases the goal-relevance of the target stimuli, it may be particularly effective at reducing goal-driven biases to positive stimuli, relative to other manipulations of control.

Further research that directly compares the influence of different types of manipulations of control on distraction from positive and negative stimuli will shed light on the systematic factors that determine whether positive and negative distraction are reduced. Moreover, manipulating the motivational value of the emotional distractors, for example, food stimuli in hungry and satiated people, or smoking-related stimuli in smokers under different levels of craving, will help to elucidate the influence of the motivational relevance of distractors on our ability to control our attention to them. Reward may be particularly effective at reducing attention to distractors with high current motivational relevance (e.g., cigarette stimuli in a deprived smoker).

\section{Some thoughts on reward}

Reward has multiple components (Berridge \& Robinson, 1998), which are thought to have different influences on cognitive control (Notebaert \& Braem, 2016). In Notebaert and Braem's model, the learning component of reward is thought to increase task engagement and exploitation of currently available rewards; the motivational component of reward is thought to enhance proactive control; and the affective component of reward is thought to increase exploration, thus reducing proactive control. Thus, according to this model, the learning and 


\section{Motivation and Emotional Distraction}

motivational components of reward should reduce distraction; while the affective component of reward should increase distraction. In my task, both subcomponents of reward learning instrumental and associative conditioning - could be at play. Providing reward for enhanced attention to the letter targets is a form of positive reinforcement (Thorndike, 1927), which increases the likelihood of attending to them on subsequent trials, in order to maximise future rewards. Furthermore, pairing the letter targets with reward could also elicit associative conditioning, imbuing them with positive emotional value, which may bias attention towards them. According to Notebaert and Braem's (2016) model, this reward learning would increase task engagement to exploit currently available rewards. The motivational, "wanting" component of reward is thought to increase exploitation of the environment, promoting proactive control to reduce distraction. In contrast, the affective component of reward may actually counteract the beneficial influence of reward on control of distraction; the affective component of reward is thought to encourage exploration of the environment, increasing distractibility.

Thus, the motivational component and the affective component of reward may have directly opposing influences on proactive control (Chiew \& Braver, 2011; Fröber \& Dreisbach, 2014; Notebaert \& Braem, 2016; but see Chiew \& Braver, 2014). But findings from studies that have induced positive affect or provided randomly-delivered, nonperformance contingent reward (which dissociates the affective and motivational components) are mixed; positive affect can reduce proactive control (Fröber \& Dreisbach, 2014), but in other contexts, can increase proactive control (Chiew \& Braver, 2014). My reward manipulation included a combination of immediate, affectively positive feedback (a bell sound ${ }^{8}$ ) signalling accumulation of points; short-term goals of reaching levels; performance feedback (on mean RTs and accuracy); encouragement (e.g., "remember to keep trying to be fast and accurate to get to the next level!"); and sounds and animations between blocks (coins falling into piles). I used these various components of the reward manipulation to maximise the impact of reward on distraction. Providing immediate feedback (the bell sound), and information on progress towards goals between blocks, are thought to increase motivation (Richter, Raban, \& Rafaeli, 2015). But it is possible that the affectively positive nature of the feedback and encouragement could actually have reduced the impact of the motivational manipulation.

\footnotetext{
${ }^{8}$ Experiment 2 did not include the bell sound because of its possible impact on pupil dilation. Visual feedback was provided instead, to indicate whether reward was attained on each trial.
} 
Motivation and Emotional Distraction

Determining the individual (and additive) influence of each of the components of the reward manipulation would be an interesting and productive line of future research. For example, future work examining the influence of performance-contingent versus performance non-contingent reward would help to disentangle the influence of the motivational component of reward on control. Studies examining the influence of affectively neutral versus affectively positive feedback would be useful for determining how the affective component of reward influences control.

\section{Limitations and future directions}

As is necessary when designing cognitive experiments, I made a number of considered experimental design decisions. These decisions were made for specific reasons, but naturally come with costs to generalisability. Below I will outline the reasons for these decisions - including use of RT deadlines, stimulus selection, use of valence blocking, and use of between-subjects reward - their associated limitations, and ideas for future extensions to address those limitations.

RT deadlines are commonly used in reward experiments (Botvinick \& Braver, 2015; Yee \& Braver, 2018), and are an effective way of eliciting motivation. The optimal response is to be both fast and accurate, for which you gain reward. In all experiments, imposing an RT deadline to gain reward induced a strategy of making slightly more errors in order to substantially speed up responses. This strategy is beneficial for maximising reward. When a task has two response options and the payoff scheme depends on fast responses, if you respond quickly, at random, you still have a 50\% chance of reward; but if you are accurate and too slow you have $0 \%$ chance of reward. Examining inverse efficiency distraction indices (which penalise RTs for errors, thus compensating for the tradeoff; Bruyer \& Brysbaert, 2011) indicated that, in Experiment 3, some, but not all, of the influence of reward on control of distraction may have been due to this change in response strategy rather than pure upregulation of control. However, in the three other experiments (which were higherpowered), the effect of reward on inverse efficiency distraction remained significant. Thus, a simple strategy of responding quickly at the cost of accuracy does not account for all of the influence of motivation on control of emotional distraction. Rather, motivation elicits upregulation of cognitive control processes, allowing people to better ignore emotional distractions. Future studies could emphasise accuracy over speed, or impose a threshold level of accuracy across the task, to prevent a speed-accuracy tradeoff from occurring.

I note that in the baseline blocks distractors were intact neutral (Experiment 1), or scrambled (Experiments 2, 3, and 4) images. Thus, the median RT threshold was based on 


\section{Motivation and Emotional Distraction}

performance on non-emotional distractor trials, creating a more stringent contingency for reward on emotional than on neutral distractor trials in subsequent experimental blocks. This raises the possibility that this criterion was not an effective continency for reward on neutral distractor trials, which could explain the greater influence of reward on emotional than neutral distraction. However, analyses of the mean proportion of rewarded trials for each valence and distractor type condition (see Appendix G, Table S.2), show that the median RT threshold was indeed an effective reward contingency even on neutral distractor blocks. In future studies, it should be considered whether separate reward criteria should be used for each distractor valence, based on median RTs on trials of that distractor valence.

The distractor images were carefully selected from the IAPS (Lang et al., 2008) so that the positive and negative images were matched on high subjective arousal ratings; and were as low (for the negative set) and high (for the positive set) on subjective valence ratings as possible. They were also selected to control for perceptual factors (e.g., not including images with black borders), the sets were limited to 12 images each. In order to include sufficient trial numbers to obtain stable RT means, this meant repeating the images across the experiment. Thus, participants may have habituated to viewing the same images throughout the experiment. However, ERP findings show that even when images are presented up to 90 times in the same session, (Codispoti, Ferrari, \& Bradley, 2007), emotional images continue to elicit a larger LPP (indexing elaborative processing of emotional content). Emotional modulation of the EPN (indexing early perceptual and attentional biases to emotion) is also not influenced by repetition (Schupp et al., 2006). A supplementary analysis for Experiment 1 (see Appendix H) showed that, although distraction did reduce on second repetition of the images, importantly this was not influenced by reward. Thus, although repetition may elicit some habituation, this did not affect the beneficial influence of reward on control of distraction.

The stimulus sets were also limited to high arousal negative (mutilation) and positive (erotic) images, because they are known to produce robust attentional biases (e.g., Grimshaw et al., 2018; Gupta et al., 2016; Most et al., 2007; Padmala et al., 2018) and large increases in pupil dilation (Bradley et al., 2008; Henderson et al., 2014). Extension to other stimulus categories (e.g., babies, food, threatening animals), with varied motivational value, will be necessary to determine how well these effects generalise. Moreover, the erotic image set included only heterosexual couples, and I did not select participants based on their sexual orientation. The subjective emotional value of both erotic and mutilation images naturally differs across people, with sexual orientation being one influencing factor. Although random 
Motivation and Emotional Distraction

assignment to reward groups should mean these individual differences cannot account for my experimental findings, such variability might be useful to explore in future work. Like the negative and positive images, the neutral images all contained people. The use of sociallyrelevant neutral images may have masked potential reward by emotion interactions that may emerge in future studies that use of more mundane neutral distractors (e.g., household objects).

The decision to block distractors by valence allowed participants to predict the valence of a potential distractor (but not when one would occur). Valence blocking enabled me to minimise carry-over effects of distractor valence on subsequent trials, so as to demonstrate the effect of reward on positive, negative, and neutral distraction separately. Blocking also allowed me to examine tonic and phasic proactive pupil dilation separately for emotional versus neutral blocks of trials, to examine the influence of expected distractor valence on adjustments to proactive control. To the extent that expectations influence the mechanisms used to control emotional and neutral distractions, it is possible that reward might be similarly effective for controlling positive and negative distraction, and less effective for controlling neutral distraction, only when distractor valence is predictable. Designs in which distractor valence is intermixed and unpredictable will be useful in future work to examine how expectations affect control of emotional distraction.

The between-subjects, blocked reward manipulation -intended to maximise the impact of reward - resulted in a strong test of whether motivation can affect sustained control of negative and positive distractions. My manipulation contrasts with that of Padmala and colleagues (Padmala \& Pessoa, 2014; Padmala et al., 2017), who manipulated reward withinsubjects, via pre-trial cues. Their findings therefore reflect a dynamic, trial-by-trial shift in control of negative distraction. Future research should address whether these dynamic shifts are also possible when controlling positive distractions.

\section{Applications}

My thesis shows that motivation can improve control of emotional distractions, both positive and negative. Elucidating how we can voluntarily exert control over emotional distractions may be useful for understanding and treating various mental disorders. Extreme attentional biases to emotion, and difficulty controlling attention, may contribute to the development and maintenance of various psychopathologies. For example, biases towards threatening and depressive stimuli may contribute to the development and maintenance of clinical anxiety and depression, respectively (Bar-Haim et al., 2007; De Raedt \& Koster, 


\section{Motivation and Emotional Distraction}

2010; Eysenck, Derakshan, Santos, \& Calvo, 2007; Mathews \& MacLeod, 2005; Mogg et al., 1995). Similarly, individuals with eating disorders show biases towards food, body shape, and weight-related information (Faunce, 2002; Lee \& Shafran, 2004). On the other hand, biases towards appetitive stimuli characterise disorders of excess. Biases towards motivationally-relevant information are found in obese individuals (Yokum, Ng, \& Stice, 2011); problem gamblers (Hønsi, Mentzoni, Molde, \& Pallesen, 2013); smokers (Ehrman et al., 2002); alcohol dependents (Field, Mogg, Zetteler, \& Bradley, 2004); and substance abusers (Field \& Cox, 2008). These biases may play a causal role in substance use (Field \& Eastwood, 2005) and difficulty quitting (Bradley, Mogg, Wright, \& Field, 2003). It is therefore important to elucidate the cognitive mechanisms underpinning emotional biases to better understand and treat these disorders. In addition to attentional biases, mood disorders and substance abuse are also associated with difficulties with attentional control (Eysenck et al., 2007; Garavan \& Hester, 2007; Jones et al., 2015). For example, the Attentional Control Theory proposes that anxiety enhances the impact of exogenous, stimulus-driven factors (i.e., enhances the salience of threatening stimuli), and decreases the impact of endogenous factors driving attention to goal-relevant information (Eysenck et al., 2007). But, attentional biases in psychopathology are also influenced by the motivational value of the stimulus (e.g., the extent to which someone craves a drug; Franken, 2003; Ryan, 2002). Increasing task motivation may therefore be particularly effective at enhancing attention to currently goalrelevant information to control disorder-relevant distractions. The extent to which attentional biases in psychopathology are driven by exogenous factors, endogenous factors, and with failures of attentional control, are not yet clear. Here we examined the influence of motivation - a manipulation that influences endogenous attention, and attentional control - in non-clinical samples. Similar methods could be used in clinical samples to examine how these factors influence attentional biases to disorder-relevant stimuli.

Current findings also may be applied to enhancing performance on a variety of everyday tasks. We are often distracted by emotional things in our day-to-day lives. For example, understanding the factors that can enhance task performance, and decrease emotional distraction, would be useful for enhancing sports performance, increasing driving safety, and improving productivity in the workplace. This is especially relevant for professions that come with a degree of risk, for example, people in the military, doctors, or pilots. In these professions, emotional distractions could be particularly dangerous. Elucidating the mechanisms by which emotional distraction occurs in these contexts, and the extent to which they can be voluntarily controlled, will be fruitful in future work. 


\section{Motivation and Emotional Distraction}

\section{Conclusions}

Attentional biases to emotional stimuli are crucial for our survival. But when emotional stimuli are neither imperative, nor relevant to current goals, we face the challenge of ignoring them. In these situations, cognitive control mechanisms are necessary to allow us to attend to currently relevant information to successfully achieve our goals. In emotionallyneutral contexts, motivation elicits a shift to using more effective proactive control mechanisms to shield us from distractions. But, little is known about the factors that can enhance control of emotional distractions. My thesis examined whether, and how, motivation can reduce emotional distraction by enhancing cognitive control. My findings definitively show that motivation, elicited by performance-contingent reward, is one way of enhancing control over both negative and positive distractions.

Motivation enhances control of emotional distractions that appear within the current spatial locus of attention (centrally); as well as those that appear outside the current spatial locus of attention (peripherally). Futhermore, despite their opposing influences on motivation, distraction from positive and negative stimuli were similarly reduced by reward; and emotional distraction was reduced to a greater extent than neutral distraction. This finding is consistent with the hypothesis that reward enhances goal-driven attention to the target stimuli by enhancing their motivational value, altering the outcome of goal-driven attentional competition between targets and distractors. However, further research is necessary to determine the mechanisms by which motivation enhances control of emotional and neutral distractions. Pupil dilation findings suggest that control of centrally-presented emotional distractions occurs via dynamic upregulation of proactive control mechanisms to facilitate rapid disengagement. Moreover, when emotional distractors are expected, sustained proactive control is enhanced to reduce distraction, but this sustained control is not modulated by reward. Finally, motivation-elicited control can be induced by a non-monetary reward manipulation.

My findings show that motivation can elicit better control of task-irrelevant positive and negative emotional stimuli, despite them being highly distracting. The relationship between emotion, motivation, and cognitive control is complex (Okon-singer et al., 2015; Pessoa, 2009), and research examining their interplay is in its infancy. Further exploration of their interactions will provide richer understanding of how motivation and emotion interact to allow us to successfully navigate the world. 
Motivation and Emotional Distraction

\section{References}

Amaral, D. G., Behniea, H., \& Kelly, J. L. (2003). Topographic organization of projections from the amygdala to the visual cortex in the macaque monkey. Neuroscience, 118(4), 1099-1120. https://doi.org/10.1016/S0306-4522(02)01001-1

Anderson, A. K., Christoff, K., Panitz, D., Rosa, E. D., \& Gabrieli, J. D. E. (2003). Neural correlates of the automatic processing of threat facial signals. Journal of Neuroscience, 23(13), 5627-5633. https://doi.org/10.1523/JNEUROSCI.23-1305627.2003

Anderson, A. K., \& Phelps, E. A. (2001). Lesions of the human amygdala impair enhanced perception of emotionally salient events. Nature, 411(6835), 305-309. https://doi.org/10.1038/35077083

Anderson, B. A. (2013). A value-driven mechanism of attentional selection. Journal of Vision, 13(3), 7-7. https://doi.org/10.1167/13.3.7

Anderson, B. A., Laurent, P. A., \& Yantis, S. (2011). Value-driven attentional capture. Proceedings of the National Academy of Sciences, 108(25), 10367-10371. https://doi.org/10.1073/pnas.1104047108

Anderson, B. A., \& Yantis, S. (2013). Persistence of value-driven attentional capture. Journal of Experimental Psychology: Human Perception and Performance, 39(1), 6-9. https://doi.org/10.1037/a0030860

Armony, J. L., \& Dolan, R. J. (2002). Modulation of spatial attention by fear-conditioned stimuli: an event-related fMRI study. Neuropsychologia, 40(7), 817-826. https://doi.org/10.1016/S0028-3932(01)00178-6

Armstrong, R. A. (2014). When to use the Bonferroni correction. Ophthalmic and Physiological Optics, 34(5), 502-508. https://doi.org/10.1111/opo.12131

Ashby, F. G., Isen, A. M., \& Turken, A. U. (1999). A Neuropsychological theory of positive affect and its ifluence on cognition. Psychological Review, 106(3), 529-550.

Augst, S., Kleinsorge, T., \& Kunde, W. (2014). Can we shield ourselves from task disturbance by emotion-laden stimulation? Cognitive, Affective, \& Behavioral Neuroscience, 14(3), 1009-1025. https://doi.org/10.3758/s13415-013-0243-x

Awh, E., Belopolsky, A. V., \& Theeuwes, J. (2012). Top-down versus bottom-up attentional control: a failed theoretical dichotomy. Trends in Cognitive Sciences, 16(8), 437-443. https://doi.org/10.1016/j.tics.2012.06.010

Barch, D. M., Braver, T. S., Nystrom, L. E., Forman, S. D., Noll, D. C., \& Cohen, J. D. (1997). Dissociating working memory from task difficulty in human prefrontal cortex. 
Motivation and Emotional Distraction

Neuropsychologia, 35(10), 1373-1380. https://doi.org/10.1016/S00283932(97)00072-9

Bar-Haim, Y., Lamy, D., Pergamin, L., Bakermans-Kranenburg, M. J., \& van IJzendoorn, M. H. (2007). Threat-related attentional bias in anxious and nonanxious individuals: a meta-analytic study. Psychological Bulletin, 133(1), 1-24. https://doi.org/10.1037/0033-2909.133.1.1

Beatty, J., \& Lucero-Wagoner, B. (2000). The pupillary system. In Handbook of psychophysiology, 2nd ed (pp. 142-162). New York, NY, US: Cambridge University Press.

Beck, D. M., \& Kastner, S. (2005). Stimulus context modulates competition in human extrastriate cortex. Nature Neuroscience, 8(8), 1110-1116. https://doi.org/10.1038/nn1501

Beck, D. M., \& Kastner, S. (2009). Top-down and bottom-up mechanisms in biasing competition in the human brain. Vision Research, 49(10), 1154-1165. https://doi.org/10.1016/j.visres.2008.07.012

Beck, D. M., \& Lavie, N. (2005). Look here but ignore what you see: effects of distractors at fixation. Journal of Experimental Psychology: Human Perception and Performance, 31(3), 592-607. https://doi.org/10.1037/0096-1523.31.3.592

Beck, S. M., Locke, H. S., Savine, A. C., Jimura, K., \& Braver, T. S. (2010). Primary and secondary rewards differentially modulate neural activity dynamics during working memory. PLOS ONE, 5(2), e9251. https://doi.org/10.1371/journal.pone.0009251

Belopolsky, A. V. (2015). Common priority map for selection history, reward and emotion in the oculomotor system. Perception, 44(8-9), 920-933. https://doi.org/10.1177/0301006615596866

Belopolsky, A. V., Schreij, D., \& Theeuwes, J. (2010). What is top-down about contingent capture? Attention, Perception, \& Psychophysics, 72(2), 326-341. https://doi.org/10.3758/APP.72.2.326

Berridge, K. C., \& Robinson, T. E. (1998). What is the role of dopamine in reward: hedonic impact, reward learning, or incentive salience? Brain Research. Brain Research Reviews, 28(3), 309-369. https://doi.org/10.1016/S0165-0173(98)00019-8

Berridge, K. C. (2012). From prediction error to incentive salience: mesolimbic computation of reward motivation. European Journal of Neuroscience, 35(7), 1124-1143. https://doi.org/10.1111/j.1460-9568.2012.07990.x 
Motivation and Emotional Distraction

Berridge, K. C., \& Robinson, T. E. (2003). Parsing reward. Trends in Neurosciences, 26(9), 507-513. https://doi.org/10.1016/S0166-2236(03)00233-9

Bickel, W. K., Pitcock, J. A., Yi, R., \& Angtuaco, E. J. C. (2009). Congruence of BOLD response across intertemporal choice conditions: fictive and real money gains and losses. Journal of Neuroscience, 29(27), 8839-8846. https://doi.org/10.1523/JNEUROSCI.5319-08.2009

Bocanegra, B. R., \& Zeelenberg, R. (2009). Emotion improves and impairs early vision. Psychological Science, 20(6), 707-713. https://doi.org/10.1111/j.14679280.2009.02354.x

Boehler, C. N., Schevernels, H., Hopf, J.-M., Stoppel, C. M., \& Krebs, R. M. (2014). Reward prospect rapidly speeds up response inhibition via reactive control. Cognitive, Affective, \& Behavioral Neuroscience, 14(2), 593-609. https://doi.org/10.3758/s13415-014-0251-5

Botvinick, M., \& Braver, T. (2015). Motivation and cognitive control: from behavior to neural mechanism. Annual Review of Psychology, 66(1), 83-113. https://doi.org/10.1146/annurev-psych-010814-015044

Botvinick, M. M., Braver, T. S., Barch, D. M., Carter, C. S., \& Cohen, J. D. (2001). Conflict monitoring and cognitive control. Psychological Review, 108(3), 624-652.

Bouret, S., \& Richmond, B. J. (2015). Sensitivity of locus ceruleus neurons to reward value for goal-directed actions. The Journal of Neuroscience, 35(9), 4005-4014. https://doi.org/10.1523/JNEUROSCI.4553-14.2015

Bowen, H. J., \& Kensinger, E. A. (2017). Cash or credit? Compensation in psychology studies: motivation matters. Collabra: Psychology, 3(1). https://doi.org/10.1525/collabra.77

Bradley, B. P., Mogg, K., Wright, T., \& Field, M. (2003). Attentional bias in drug dependence: vigilance for cigarette-related cues in smokers. Psychology of Addictive Behaviors, 17(1), 66-72. https://doi.org/10.1037/0893-164X.17.1.66

Bradley, M. M. (2009). Natural selective attention: orienting and emotion. Psychophysiology, 46(1), 1-11. https://doi.org/10.1111/j.1469-8986.2008.00702.x

Bradley, M. M., Codispoti, M., Cuthbert, B. N., \& Lang, P. J. (2001). Emotion and motivation I: defensive and appetitive reactions in picture processing. Emotion, 1(3), 276-298. https://doi.org/10.1037//1528-3542.1.3.276 
Motivation and Emotional Distraction

Bradley, M. M., Miccoli, L., Escrig, M. A., \& Lang, P. J. (2008). The pupil as a measure of emotional arousal and autonomic activation. Psychophysiology, 45(4), 602-607. https://doi.org/10.1111/j.1469-8986.2008.00654.x

Braver, T. S. (2012). The variable nature of cognitive control: a dual mechanisms framework. Trends in Cognitive Sciences, 16(2), 106-113. https://doi.org/10.1016/j.tics.2011.12.010

Braver, T. S., Gray, J. R., \& Burgess, G. C. (2007). Explaining the many varieties of working memory variation: dual mechanisms of cognitive control. In Variation in working memory (pp. 76-106). New York, NY, US: Oxford University Press.

Braver, T. S., Paxton, J. L., Locke, H. S., \& Barch, D. M. (2009). Flexible neural mechanisms of cognitive control within human prefrontal cortex. Proceedings of the National Academy of Sciences, pnas.0808187106. https://doi.org/10.1073/pnas.0808187106

Bray, S., Shimojo, S., \& O’Doherty, J. P. (2010). Human medial orbitofrontal cortex is recruited during experience of imagined and real rewards. Journal of Neurophysiology, 103(5), 2506-2512. https://doi.org/10.1152/jn.01030.2009

Brisson, J., Mainville, M., Mailloux, D., Beaulieu, C., Serres, J., \& Sirois, S. (2013). Pupil diameter measurement errors as a function of gaze direction in corneal reflection eyetrackers. Behavior Research Methods, 45(4), 1322-1331. https://doi.org/10.3758/s13428-013-0327-0

Broadbent, D. E., \& Broadbent, M. H. P. (1987). From detection to identification: response to multiple targets in rapid serial visual presentation. Perception \& Psychophysics, 42(2), 105-113. https://doi.org/10.3758/BF03210498

Brosch, T., Pourtois, G., \& Sander, D. (2010). The perception and categorisation of emotional stimuli: A review. Cognition and Emotion, 24(3), 377-400. http://dx.doi.org/10.1080/02699930902975754

Brosch, T., Pourtois, G., Sander, D., \& Vuilleumier, P. (2011). Additive effects of emotional, endogenous, and exogenous attention: behavioral and electrophysiological evidence. Neuropsychologia, 49(7), 1779-1787. https://doi.org/10.1016/j.neuropsychologia.2011.02.056

Brosch, T., Sander, D., Pourtois, G., \& Scherer, K. R. (2008). Beyond fear: rapid spatial orienting toward positive emotional stimuli. Psychological Science, 19(4), 362-370. https://doi.org/10.1111/j.1467-9280.2008.02094.x 
Motivation and Emotional Distraction

Brosch, T., Sander, D., \& Scherer, K. R. (2007). That baby caught my eye... Attention capture by infant faces. Emotion, 7(3), 685-689. https://doi.org/10.1037/15283542.7.3.685

Brown, C. R. H., Berggren, N., \& Forster, S. (2018). Testing a goal-driven account of involuntary attentional capture by threat. Emotion. Retrieved from http://sro.sussex.ac.uk/80239/

Bruyer, R., \& Brysbaert, M. (2011). Combining speed and accuracy in cognitive psychology: is the Inverse Efficiency Score (IES) a better dependent variable than the mean Reaction Time (RT) and the Percentage of Errors (PE)? Psychologica Belgica, 51(1), $5-13$.

Bugg, J. M. (2012). Dissociating levels of cognitive control: the case of Stroop interference. Current Directions in Psychological Science, 21(5), 302-309. https://doi.org/10.1177/0963721412453586

Bugg, J. M., \& Crump, M. J. C. (2012). In support of a distinction between voluntary and stimulus-driven control: a review of the literature on proportion congruent effects. Frontiers in Psychology, 3. https://doi.org/10.3389/fpsyg.2012.00367

Burra, N., Pittet, C., Barras, C., \& Kerzel, D. (2018). Attentional suppression is delayed for threatening distractors. https://doi.org/10.31234/osf.io/329w4

Carretié, L. (2014). Exogenous (automatic) attention to emotional stimuli: a review. Cognitive, Affective, \& Behavioral Neuroscience, 14(4), 1228-1258. https://doi.org/10.3758/s13415-014-0270-2

Carver, C. (2003). Pleasure as a sign you can attend to something else: placing positive feelings within a general model of affect. Cognition and Emotion, 17(2), 241-261. https://doi.org/10.1080/02699930302294

Carver, C. S., \& White, T. L. (1994). Behavioral inhibition, behavioral activation, and affective responses to impending reward and punishment: the BIS/BAS Scales. Journal of Personality and Social Psychology, 67(2), 319-333. http://dx.doi.org/10.1037/0022-3514.67.2.319

Cepeda, N. J., Cave, K. R., Bichot, N. P., \& Kim, M.-S. (1998). Spatial selection via featuredriven inhibition of distractor locations. Perception \& Psychophysics, 60(5), 727-746. https://doi.org/10.3758/BF03206059

Chapman, A., Devue, C., \& Grimshaw, G. M. (2017). Fleeting reliability in the dot-probe task. Psychological Research. https://doi.org/10.1007/s00426-017-0947-6 
Motivation and Emotional Distraction

Chatham, C. H., Frank, M. J., \& Munakata, Y. (2009). Pupillometric and behavioral markers of a developmental shift in the temporal dynamics of cognitive control. Proceedings of the National Academy of Sciences, 106(14), 5529-5533. https://doi.org/10.1073/pnas.0810002106

Chen, Z., \& Treisman, A. (2008). Distractor inhibition is more effective at a central than at a peripheral location. Perception \& Psychophysics, 70(6), 1081-1091. https://doi.org/10.3758/PP.70.6.1081

Chevalier, N., Martis, S. B., Curran, T., \& Munakata, Y. (2015). Metacognitive processes in executive control development: the case of reactive and proactive control. Journal of Cognitive Neuroscience, 27(6), 1125-1136. https://doi.org/10.1162/jocn_a_00782

Chiew, K. S., \& Braver, T. S. (2011). Positive affect versus reward: emotional and motivational influences on cognitive control. Frontiers in Psychology, 2. https://doi.org/10.3389/fpsyg.2011.00279

Chiew, K. S., \& Braver, T. S. (2013). Temporal dynamics of motivation-cognitive control interactions revealed by high-resolution pupillometry. Frontiers in Psychology, 4. https://doi.org/10.3389/fpsyg.2013.00015

Chiew, K. S., \& Braver, T. S. (2014). Dissociable influences of reward motivation and positive emotion on cognitive control. Cognitive, Affective, \& Behavioral Neuroscience, 14(2), 509-529. https://doi.org/10.3758/s13415-014-0280-0

Chiew, K. S., \& Braver, T. S. (2016). Reward favors the prepared: incentive and taskinformative cues interact to enhance attentional control. Journal of Experimental Psychology. Human Perception and Performance, 42(1), 52-66. https://doi.org/10.1037/xhp0000129

Chun, M. M., \& Potter, M. C. (1995). A two-stage model for multiple target detection in rapid serial visual presentation. Journal of Experimental Psychology. Human Perception and Performance, 21(1), 109-127. https://doi.org/10.1037//00961523.21.1.109

Codispoti, M., Ferrari, V., \& Bradley, M. M. (2007). Repetition and event-related potentials: distinguishing early and late processes in affective picture perception. Journal of Cognitive Neuroscience, 19(4), 577-586. https://doi.org/10.1162/jocn.2007.19.4.577

Cohen, J. (1992). A power primer. Psychological Bulletin, 112(1), 155-159. http://dx.doi.org/10.1037/0033-2909.112.1.155

Cohen, J. D. (2017). Cognitive control. In The Wiley Handbook of Cognitive Control (pp. 128). Wiley-Blackwell. https://doi.org/10.1002/9781118920497.ch1 
Motivation and Emotional Distraction

Cohen, N., Henik, A., \& Mor, N. (2010). Can emotion modulate attention? Evidence for reciprocal links in the attentional network test. Experimental Psychology, 58(3), 171179. https://doi.org/10.1027/1618-3169/a000083

Cohen, N., Moyal, N., \& Henik, A. (2015). Executive control suppresses pupillary responses to aversive stimuli. Biological Psychology, 112, 1-11. https://doi.org/10.1016/j.biopsycho.2015.09.006

Corbetta, M., \& Shulman, G. L. (2002). Control of goal-directed and stimulus-driven attention in the brain. Nature Reviews Neuroscience, 3(3), 201-215. https://doi.org/10.1038/nrn755

Costa, V. D., Lang, P. J., Sabatinelli, D., Versace, F., \& Bradley, M. M. (2010). Emotional imagery: Assessing pleasure and arousal in the brain's reward circuitry. Human Brain Mapping, 31(9), 1446-1457. https://doi.org/10.1002/hbm.20948

Coull, J. T., Büchel, C., Friston, K. J., \& Frith, C. D. (1999). Noradrenergically mediated plasticity in a human attentional neuronal network. NeuroImage, 10(6), 705-715. https://doi.org/10.1006/nimg.1999.0513

Czernochowski, D. (2015). ERPs dissociate proactive and reactive control: evidence from a task-switching paradigm with informative and uninformative cues. Cognitive, Affective, \& Behavioral Neuroscience, 15(1), 117-131. https://doi.org/10.3758/s13415-014-0302-y

Dambacher, M., Hübner, R., \& Schlösser, J. (2011). Monetary incentives in speeded perceptual decision: effects of penalizing errors versus slow responses. Frontiers in Psychology, 2. https://doi.org/10.3389/fpsyg.2011.00248

Darwin, C. R. (1869). On the origin of species by means of natural selection, or the preservation of favoured races in the struggle for life. London: John Murray. 5th edition.

De Raedt, R., \& Koster, E. H. W. (2010). Understanding vulnerability for depression from a cognitive neuroscience perspective: a reappraisal of attentional factors and a new conceptual framework. Cognitive, Affective \& Behavioral Neuroscience, 10(1), 5070. https://doi.org/10.3758/CABN.10.1.50

Delacre, M., Lakens, D., \& Leys, C. (2017). Why psychologists should by default use Welch's $t$-test instead of Student's $t$-test. International Review of Social Psychology, 30(1), 92-101. https://doi.org/10.5334/irsp.82 
Motivation and Emotional Distraction

Desimone, R., \& Duncan, J. (1995). Neural mechanisms of selective visual attention. Annual Review of Neuroscience, 18(1), 193-222. https://doi.org/10.1146/annurev.ne.18.030195.001205

Dolan, R. J. (2002). Emotion, cognition, and behavior. Science, 298(5596), 1191-1194. https://doi.org/10.1126/science.1076358

Dreisbach, G., \& Goschke, T. (2004). How positive affect modulates cognitive control: reduced perseveration at the cost of increased distractibility. Journal of Experimental Psychology: Learning, Memory, and Cognition, 30(2), 343-353. https://doi.org/10.1037/0278-7393.30.2.343

Eastwood, J. D., Smilek, D., \& Merikle, P. M. (2001). Differential attentional guidance by unattended faces expressing positive and negative emotion. Perception \& Psychophysics, 63(6), 1004-1013. https://doi.org/10.3758/BF03194519

Egeth, H. E., \& Yantis, S. (1997). Visual attention: control, representation, and time course. Annual Review of Psychology, 48(1), 269-297. https://doi.org/10.1146/annurev.psych.48.1.269

Egner, T., Etkin, A., Gale, S., \& Hirsch, J. (2008). Dissociable neural systems resolve conflict from emotional versus nonemotional distracters. Cerebral Cortex, 18(6), 1475-1484. https://doi.org/10.1093/cercor/bhm179

Ehrman, R. N., Robbins, S. J., Bromwell, M. A., Lankford, M. E., Monterosso, J. R., \& O'Brien, C. P. (2002). Comparing attentional bias to smoking cues in current smokers, former smokers, and non-smokers using a dot-probe task. Drug and Alcohol Dependence, 67(2), 185-191. https://doi.org/10.1016/S0376-8716(02)00065-0

Engelmann, J. B. (2009). Combined effects of attention and motivation on visual task performance: transient and sustained motivational effects. Frontiers in Human Neuroscience, 3. https://doi.org/10.3389/neuro.09.004.2009

Erthal, F. S., De Oliveira, L., Mocaiber, I., Pereira, M. G., Machado-Pinheiro, W., Volchan, E., \& Pessoa, L. (2005). Load-dependent modulation of affective picture processing. Cognitive, Affective, \& Behavioral Neuroscience, 5(4), 388-395. https://doi.org/10.3758/CABN.5.4.388

Etzel, J. A., Cole, M. W., Zacks, J. M., Kay, K. N., \& Braver, T. S. (2016). Reward Motivation enhances task coding in frontoparietal cortex. Cerebral Cortex, 26(4), 1647-1659. https://doi.org/10.1093/cercor/bhu327 
Motivation and Emotional Distraction

Eysenck, M. W., Derakshan, N., Santos, R., \& Calvo, M. G. (2007). Anxiety and cognitive performance: attentional control theory. Emotion, 7(2), 336-353. https://doi.org/10.1037/1528-3542.7.2.336

Failing, M., \& Theeuwes, J. (2018). Selection history: How reward modulates selectivity of visual attention. Psychonomic Bulletin \& Review, 25(2), 514-538. https://doi.org/10.3758/s13423-017-1380-y

Faul, F., Erdfelder, E., Lang, A.-G., \& Buchner, A. (2007). G*Power 3: A flexible statistical power analysis program for the social, behavioral, and biomedical sciences. Behavior Research Methods, 39(2), 175-191. https://doi.org/10.3758/BF03193146

Faunce, G. J. (2002). Eating Disorders and Attentional Bias: A Review. Eating Disorders, 10(2), 125-139. https://doi.org/10.1080/10640260290081696

Fecteau, J. H., \& Munoz, D. P. (2006). Salience, relevance, and firing: a priority map for target selection. Trends in Cognitive Sciences, 10(8), 382-390. https://doi.org/10.1016/j.tics.2006.06.011

Field, M., \& Cox, W. (2008). Attentional bias in addictive behaviors: a review of its development, causes, and consequences. Drug and Alcohol Dependence, 97(1-2), 120. https://doi.org/10.1016/j.drugalcdep.2008.03.030

Field, M., \& Eastwood, B. (2005). Experimental manipulation of attentional bias increases the motivation to drink alcohol. Psychopharmacology, 183(3), 350-357. https://doi.org/10.1007/s00213-005-0202-5

Field, M., Mogg, K., Zetteler, J., \& Bradley, B. P. (2004). Attentional biases for alcohol cues in heavy and light social drinkers: the roles of initial orienting and maintained attention. Psychopharmacology, 176(1), 88-93. https://doi.org/10.1007/s00213-004$1855-1$

Field, M., Munafò, M., \& Franken, I. (2009). A meta-analytic investigation of the relationship between attentional bias and subjective craving in substance abuse. Psychological Bulletin, 135(4), 589-607. https://doi.org/10.1037/a0015843

Field, M., Werthmann, J., Franken, I., Hofmann, W., Hogarth, L., \& Roefs, A. (2016). The role of attentional bias in obesity and addiction. Health Psychology, 35(8), 767-780. https://doi.org/10.1037/hea0000405

Folk, C. L., Remington, R. W., \& Johnston, J. C. (1992). Involuntary covert orienting is contingent on attentional control settings. Journal of Experimental Psychology: Human Perception and Performance, 18(4), 1030-1044. https://doi.org/10.1037/0096-1523.18.4.1030 
Motivation and Emotional Distraction

Forster, S., \& Lavie, N. (2008). Attentional capture by entirely irrelevant distractors. Visual Cognition, 16(2-3), 200-214. https://doi.org/10.1080/13506280701465049

Fox, E., Griggs, L., \& Mouchlianitis, E. (2007). The detection of fear-relevant stimuli: are guns noticed as quickly as snakes? Emotion (Washington, D.C.), 7(4), 691-696. https://doi.org/10.1037/1528-3542.7.4.691

Fox, E., Yates, A., \& Ashwin, C. (2012). Trait anxiety and perceptual load as determinants of emotion processing in a fear conditioning paradigm. Emotion, 12(2), 236-249. https://doi.org/10.1037/a0025321

Franken, I. H. A. (2003). Drug craving and addiction: integrating psychological and neuropsychopharmacological approaches. Progress in Neuro-Psychopharmacology and Biological Psychiatry, 27(4), 563-579. https://doi.org/10.1016/S02785846(03)00081-2

Fredrickson, B. L. (2001). The role of positive emotions in positive psychology: the broadenand-build theory of positive emotions. American Psychologist, 56(3), 218-226. https://doi.org/10.1037/0003-066X.56.3.218

Fredrickson, B. L. (2013). Positive emotions broaden and build. In Advances in Experimental Social Psychology (Vol. 47, pp. 1-53). Elsevier. https://doi.org/10.1016/B978-0-12407236-7.00001-2

Frijda, N. H. (1986). The emotions. Paris, France: Editions de la Maison des Sciences de l'Homme.

Fröber, K., \& Dreisbach, G. (2014). The differential influences of positive affect, random reward, and performance-contingent reward on cognitive control. Cognitive, Affective \& Behavioral Neuroscience, 14(2), 530-547. https://doi.org/10.3758/s13415-0140259-x

Gable, P. A., Poole, B. D., \& Harmon-Jones, E. (2015). Anger perceptually and conceptually narrows cognitive scope. Journal of Personality and Social Psychology, 109(1), 163174. https://doi.org/10.1037/a0039226

Garavan, H., \& Hester, R. (2007). The role of cognitive control in cocaine dependence. Neuropsychology Review, 17(3), 337-345. https://doi.org/10.1007/s11065-007-9034$\mathrm{X}$

Gaspelin, N., Leonard, C. J., \& Luck, S. J. (2015). Direct evidence for active suppression of salient-but-irelevant sensory inputs. Psychological Science, 26(11), 1740-1750. https://doi.org/10.1177/0956797615597913 
Motivation and Emotional Distraction

Gaspelin, N., \& Luck, S. J. (2018). Combined electrophysiological and behavioral evidence for the suppression of salient distractors. Journal of Cognitive Neuroscience, 1-16. https://doi.org/10.1162/jocn_a_01279

Geng, J. J. (2014). Attentional mechanisms of distractor suppression. Current Directions in Psychological Science, 23(2), 147-153. https://doi.org/10.1177/0963721414525780

Gerber, A. J., Posner, J., Gorman, D., Colibazzi, T., Yu, S., Wang, Z., Kangarlu, A., Zhu, H., Russell, J., \& Peterson, B. S. (2008). An affective circumplex model of neural systems subserving valence, arousal, and cognitive overlay during the appraisal of emotional faces. Neuropsychologia, 46(8), 2129-2139. https://doi.org/10.1016/j.neuropsychologia.2008.02.032

Gratton, G., Coles, M., \& Donchin, E. (1992). Optimizing the use of information: strategic cntrol of activation of responses. Journal of Experimental Psychology: General, 480506. https://doi.org/10.1037/0096-3445.121.4.480

Gratton, G., Cooper, P., Fabiani, M., Carter, C. S., \& Karayanidis, F. (2018). Dynamics of cognitive control: theoretical bases, paradigms, and a view for the future. Psychophysiology, 55(3), e13016. https://doi.org/10.1111/psyp.13016

Grimshaw, G. M., Kranz, L. S., Carmel, D., Moody, R. E., \& Devue, C. (2018). Contrasting reactive and proactive control of emotional distraction. Emotion, 18(1), 26-38. https://doi.org/10.1037/emo0000337

Gupta, R., Hur, Y., \& Lavie, N. (2016). Distracted by pleasure: effects of positive versus negative valence on emotional capture under load. Emotion, 16(3), 328-337. https://doi.org/10.1037/emo0000112

Hajcak, G., Dunning, J. P., \& Foti, D. (2009). Motivated and controlled attention to emotion: time-course of the late positive potential. Clinical Neurophysiology, 120(3), 505-510. https://doi.org/10.1016/j.clinph.2008.11.028

Hajcak, G., MacNamara, A., Foti, D., Ferri, J., \& Keil, A. (2013). The dynamic allocation of attention to emotion: simultaneous and independent evidence from the late positive potential and steady state visual evoked potentials. Biological Psychology, 92(3), 447-455. https://doi.org/10.1016/j.biopsycho.2011.11.012

Harmon-Jones, E. (2003). Anger and the behavioral approach system. Personality and Individual Differences, 35(5), 995-1005. https://doi.org/10.1016/S01918869(02)00313-6

Hefer, C., \& Dreisbach, G. (2016). The motivational modulation of proactive control in a modified version of the AX-continuous performance task: evidence from cue-based 
Motivation and Emotional Distraction

and prime-based preparation. Motivation Science, 2(2), 116-134.

https://doi.org/10.1037/mot0000034

Hefer, C., \& Dreisbach, G. (2017). How performance-contingent reward prospect modulates cognitive control: Increased cue maintenance at the cost of decreased flexibility. Journal of Experimental Psychology: Learning, Memory, and Cognition, 43(10), 1643-1658. http://dx.doi.org/10.1037/xlm0000397

Heitz, R. P., Schrock, J. C., Payne, T. W., \& Engle, R. W. (2008). Effects of incentive on working memory capacity: behavioral and pupillometric data. Psychophysiology, 45(1), 119-129. https://doi.org/10.1111/j.1469-8986.2007.00605.x

Henderson, R. R., Bradley, M. M., \& Lang, P. J. (2014). Modulation of the initial light reflex during affective picture viewing: the light reflex during affective picture viewing. Psychophysiology, 51(9), 815-818. https://doi.org/10.1111/psyp.12236

Hickey, C., Chelazzi, L., \& Theeuwes, J. (2010). Reward changes salience in human vision via the anterior cingulate. Journal of Neuroscience, 30(33), 11096-11103. https://doi.org/10.1523/JNEUROSCI.1026-10.2010

Hickey, C., \& van Zoest, W. (2012). Reward creates oculomotor salience. Current Biology, 22(7), R219-R220. https://doi.org/10.1016/j.cub.2012.02.007

Holland, P. C., \& Gallagher, M. (1999). Amygdala circuitry in attentional and representational processes. Trends in Cognitive Sciences, 3(2), 65-73. https://doi.org/10.1016/S1364-6613(98)01271-6

Hønsi, A., Mentzoni, R. A., Molde, H., \& Pallesen, S. (2013). Attentional bias in problem gambling: a systematic review. Journal of Gambling Studies, 29(3), 359-375. https://doi.org/10.1007/s10899-012-9315-Z

Hopf, J. M., Schoenfeld, M. A., Buschschulte, A., Rautzenberg, A., Krebs, R. M., \& Boehler, C. N. (2015). The modulatory impact of reward and attention on global feature selection in human visual cortex. Visual Cognition, 23(1-2), 229-248. https://doi.org/10.1080/13506285.2015.1011252

Hopkins, L. S., Helmstetter, F. J., \& Hannula, D. E. (2016). Eye movements are captured by a perceptually simple conditioned stimulus in the absence of explicit contingency knowledge. Emotion, 16(8), 1157-1171. https://doi.org/10.1037/emo0000206

Hu, K., Padmala, S., \& Pessoa, L. (2013). Interactions between reward and threat during visual processing. Neuropsychologia, 51(9), 1763-1772. https://doi.org/10.1016/j.neuropsychologia.2013.05.025 
Motivation and Emotional Distraction

Hübner, R., \& Schlösser, J. (2010). Monetary reward increases attentional effort in the flanker task. Psychonomic Bulletin \& Review, 17(6), 821-826. https://doi.org/10.3758/PBR.17.6.821

Ihssen, N., \& Keil, A. (2009). The costs and benefits of processing emotional stimuli during rapid serial visual presentation. Cognition and Emotion, 23(2), 296-326. https://doi.org/10.1080/02699930801987504

Itti, L., \& Koch, C. (2000). A saliency-based search mechanism for overt and covert shifts of visual attention. Vision Research, 40(10), 1489-1506. https://doi.org/10.1016/S00426989(99)00163-7

Jackson, I., \& Sirois, S. (2009). Infant cognition: going full factorial with pupil dilation. Developmental Science, 12(4), 670-679. https://doi.org/10.1111/j.14677687.2008.00805.x

jamovi project (2018). jamovi (Version 0.9) [Computer Software]. Retrieved from https://www.jamovi.org

Jimura, K., Locke, H. S., \& Braver, T. S. (2010). Prefrontal cortex mediation of cognitive enhancement in rewarding motivational contexts. Proceedings of the National Academy of Sciences, 201002007. https://doi.org/10.1073/pnas.1002007107

Jones, N. P., Siegle, G. J., \& Mandell, D. (2015). Motivational and emotional influences on cognitive control in depression: a pupillometry study. Cognitive, Affective, \& Behavioral Neuroscience, 15(2), 263-275. https://doi.org/10.3758/s13415-014-03236

Joshi, S., Li, Y., Kalwani, R. M., \& Gold, J. I. (2016). Relationships between pupil diameter and neuronal activity in the locus coeruleus, colliculi, and cingulate cortex. Neuron, 89(1), 221-234. https://doi.org/10.1016/j.neuron.2015.11.028

Juth, P., Lundqvist, D., Karlsson, A., \& Öhman, A. (2005). Looking for foes and friends: perceptual and emotional factors when finding a face in the crowd. Emotion, 5(4), 379-395. https://doi.org/10.1037/1528-3542.5.4.379

Kahneman, D. (1973). Attention and effort. Englewood Cliffs, N.J: Prentice-Hall.

Kastner, S., \& Ungerleider, L. G. (2001). The neural basis of biased competition in human visual cortex. Neuropsychologia, 39(12), 1263-1276. https://doi.org/10.1016/S00283932(01)00116-6

Kastner, S., Weerd, P. D., Desimone, R., \& Ungerleider, L. G. (1998). Mechanisms of directed attention in the human extrastriate cortex as revealed by functional MRI. Science, 282(5386), 108-111. https://doi.org/10.1126/science.282.5386.108 
Motivation and Emotional Distraction

Kennedy, B. L., Newman, V. E., \& Most, S. B. (2017). Proactive deprioritization of emotional distractors enhances target perception. Emotion, No Pagination SpecifiedNo Pagination Specified. https://doi.org/10.1037/emo0000362

Kinner, V. L., Kuchinke, L., Dierolf, A. M., Merz, C. J., Otto, T., \& Wolf, O. T. (2017). What our eyes tell us about feelings: tracking pupillary responses during emotion regulation processes: pupillary responses during emotion regulation. Psychophysiology, 54(4), 508-518. https://doi.org/10.1111/psyp.12816

Kool, W., Shenhav, A., \& Botvinick, M. M. (2017). Cognitive control as cost-benefit decision making. In The Wiley Handbook of Cognitive Control (pp. 167-189). WileyBlackwell. https://doi.org/10.1002/9781118920497.ch10

Koster, E. H. W., Verschuere, B., Burssens, B., Custers, R., \& Crombez, G. (2007). Attention for emotional faces under restricted awareness revisited: do emotional faces automatically attract attention? Emotion, 7(2), 285-295. https://doi.org/10.1037/15283542.7.2.285

Krebs, R. M., Boehler, C. N., \& Woldorff, M. G. (2010). The influence of reward associations on conflict processing in the Stroop task. Cognition, 117(3), 341-347. https://doi.org/10.1016/j.cognition.2010.08.018

Krolak-Salmon, P., Fischer, C., Vighetto, A., \& Mauguière, F. (2001). Processing of facial emotional expression: spatio-temporal data as assessed by scalp event-related potentials. European Journal of Neuroscience, 13(5), 987-994. https://doi.org/10.1046/j.0953-816x.2001.01454.x

Krug, M. K., \& Braver, T. S. (2014). Motivation and cgnitive control: going beyond monetary incentives. In E. Bijleveld \& H. Aarts (Eds.), The Psychological Science of Money (pp. 137-162). New York, NY: Springer New York. https://doi.org/10.1007/978-1-4939-0959-9_7

Laeng, B., Sirois, S., \& Gredebäck, G. (2012). Pupillometry: a window to the preconscious? Perspectives on Psychological Science, 7(1), 18-27. https://doi.org/10.1177/1745691611427305

Lakens, D. (2013). Calculating and reporting effect sizes to facilitate cumulative science: a practical primer for t-tests and ANOVAs. Frontiers in Psychology, 4. https://doi.org/10.3389/fpsyg.2013.00863

Lane, R. D., Reiman, E. M., Axelrod, B., Yun, L.-S., Holmes, A., \& Schwartz, G. E. (1998). Neural correlates of levels of emotional awareness: Evidence of an interaction 
Motivation and Emotional Distraction

between emotion and attention in the anterior cingulate cortex. Journal of Cognitive Neuroscience, 10(4), 525-535. https://doi.org/10.1162/089892998562924

Lang, P. J., \& Bradley, M. M. (2010). Emotion and the motivational brain. Biological Psychology, 84(3), 437-450. https://doi.org/10.1016/j.biopsycho.2009.10.007

Lang, P.J., Bradley, M.M., \& Cuthbert, B.N. (2008). International affective picture system (IAPS): affective ratings of pictures and instruction manual. Technical Report A-8. University of Florida, Gainesville, FL.

Lang, P. J., Greenwald, M. K., Bradley, M. M., \& Hamm, A. O. (1993). Looking at pictures: Affective, facial, visceral, and behavioral reactions. Psychophysiology, 30(3), 261273. https://doi.org/10.1111/j.1469-8986.1993.tb03352.x

Lavie, N. (2005). Distracted and confused?: selective attention under load. Trends in Cognitive Sciences, 9(2), 75-82. https://doi.org/10.1016/j.tics.2004.12.004

Lavie, N., Hirst, A., de Fockert, J. W., \& Viding, E. (2004). Load theory of selective attention and cognitive control. Journal of Experimental Psychology. General, 133(3), 339354. https://doi.org/10.1037/0096-3445.133.3.339

Le Pelley, M. E., Pearson, D., Griffiths, O., \& Beesley, T. (2015). When goals conflict with values: counterproductive attentional and oculomotor capture by reward-related stimuli. Journal of Experimental Psychology: General, 144(1), 158-171. https://doi.org/10.1037/xge0000037

LeDoux, J. E. (1996). The emotional brain: the mysterious underpinnings of emotional life. New York: Simon \& Schuster.

LeDoux, J. E. (2000). Emotion Circuits in the Brain. Annual Review of Neuroscience, 23(1), 155-184. https://doi.org/10.1146/annurev.neuro.23.1.155

LeDoux, J. E. (2012). Rethinking the Emotional Brain. Neuron, 73(4), 653-676. https://doi.org/10.1016/j.neuron.2012.02.004

Lee, M., \& Shafran, R. (2004). Information processing biases in eating disorders. Clinical Psychology Review, 24(2), 215-238. https://doi.org/10.1016/j.cpr.2003.10.004

Lichtenstein-Vidne, L., Henik, A., \& Safadi, Z. (2012). Task relevance modulates processing of distracting emotional stimuli. Cognition and Emotion, 26(1), 42-52. https://doi.org/10.1080/02699931.2011.567055

Locke, H. S., \& Braver, T. S. (2008). Motivational influences on cognitive control: behavior, brain activation, and individual differences. Cognitive, Affective, \& Behavioral Neuroscience, 8(1), 99-112. https://doi.org/10.3758/CABN.8.1.99 
Motivation and Emotional Distraction

Luck, S. J., \& Kappenman, E. S. (2011). ERP components and selective attention. The Oxford Handbook of Event-Related Potential Components. https://doi.org/10.1093/oxfordhb/9780195374148.013.0144

Luck, S. J., Woodman, G. F., \& Vogel, E. K. (2000). Event-related potential studies of attention. Trends in Cognitive Sciences, 4(11), 432-440. https://doi.org/10.1016/S1364-6613(00)01545-X

March, J. G. (1991). Exploration and exploitation in organizational learning. Organization Science, 2(1), 71-87. https://doi.org/10.1287/orsc.2.1.71

Mather, M., \& Sutherland, M. R. (2011). Arousal-biased competition in perception and memory. Perspectives on Psychological Science: A Journal of the Association for Psychological Science, 6(2), 114-133. https://doi.org/10.1177/1745691611400234

Mathews, A., \& MacLeod, C. (2005). Cognitive vulnerability to emotional disorders. Annual Review of Clinical Psychology, 1(1), 167-195. https://doi.org/10.1146/annurev.clinpsy.1.102803.143916

Mathewson, K. J., Arnell, K. M., \& Mansfield, C. A. (2008). Capturing and holding attention: the impact of emotional words in rapid serial visual presentation. Memory \& Cognition, 36(1), 182-200. https://doi.org/10.3758/MC.36.1.182

Mathôt, S., Fabius, J., VanHeusden, E., \& Stigchel, S. V. (2017). Safe and sensible baseline correction of pupil-size data. PeerJ PrePrints; San Diego. http://dx.doi.org/10.7287/peerj.preprints.2725v1

Mathôt, S., \& Van der Stigchel, S. (2015). New light on the mind's eye: the pupillary light response as active vision. Current Directions in Psychological Science, 24(5), 374378. https://doi.org/10.1177/0963721415593725

Miller, E. K. (2000). The prefontral cortex and cognitive control. Nature Reviews Neuroscience, 1(1), 59-65. https://doi.org/10.1038/35036228

Miyapuram, K. P., Tobler, P. N., Gregorios-Pippas, L., \& Schultz, W. (2012). BOLD responses in reward regions to hypothetical and imaginary monetary rewards. NeuroImage, 59(2), 1692-1699. https://doi.org/10.1016/j.neuroimage.2011.09.029

Mogg, K., Bradley, B. P., \& Williams, R. (1995). Attentional bias in anxiety and depression: the role of awareness. British Journal of Clinical Psychology, 34(1), 17-36. https://doi.org/10.1111/j.2044-8260.1995.tb01434.x

Mohanty, A., Egner, T., Monti, J. M., \& Mesulam, M.-M. (2009). Search for a threatening target triggers limbic guidance of spatial attention. Journal of Neuroscience, 29(34), 10563-10572. https://doi.org/10.1523/JNEUROSCI.1170-09.2009 
Motivation and Emotional Distraction

Mohanty, A., Gitelman, D. R., Small, D. M., \& Mesulam, M. M. (2008). The spatial attention network interacts with limbic and monoaminergic systems to modulate motivationinduced attention shifts. Cerebral Cortex, 18(11), 2604-2613. https://doi.org/10.1093/cercor/bhn021

Mohanty, A., \& Sussman, T. J. (2013). Top-down modulation of attention by emotion. Frontiers in Human Neuroscience, 7. https://doi.org/10.3389/fnhum.2013.00102

Most, S. B., Chun, M. M., Widders, D. M., \& Zald, D. H. (2005). Attentional rubbernecking: cognitive control and personality in emotion-induced blindness. Psychonomic Bulletin \& Review, 12(4), 654-661. https://doi.org/10.3758/BF03196754

Most, S. B., Smith, S. D., Cooter, A. B., Levy, B. N., \& Zald, D. H. (2007). The naked truth: positive, arousing distractors impair rapid target perception. Cognition and Emotion, 21(5), 964-981. https://doi.org/10.1080/02699930600959340

Mulckhuyse, M. (2018). The influence of emotional stimuli on the oculomotor system: a review of the literature. Cognitive, Affective, \& Behavioral Neuroscience. https://doi.org/10.3758/s13415-018-0590-8

Müller, M. M., \& Hübner, R. (2002). Can the spotlight of attention be shaped like a doughnut? Evidence from steady-state visual evoked potentials. Psychological Science, 13(2), 119-124. https://doi.org/10.1111/1467-9280.00422

Murphy, J., Devue, C., Corballis, P., \& Grimshaw, G. M. (2018). Proactive control of emotional distraction: evidence from alpha suppression. https://doi.org/10.31219/osf.io/vuk6j

Noudoost, B., Chang, M. H., Steinmetz, N. A., \& Moore, T. (2010). Top-down control of visual attention. Current Opinion in Neurobiology, 20(2), 183-190. https://doi.org/10.1016/j.conb.2010.02.003

Notebaert, W., \& Braem, S. (2016). Parsing the effects of reward on cognitive control. In T. Braver (Ed.), Motivation and cognitive control (pp. 105-122). Routledge.

Öhman, A., Flykt, A., \& Esteves, F. (2001). Emotion drives attention: detecting the snake in the grass. Journal of experimental psychology: general, 130(3), 466. https://doi.org/10.1037/0096-3445.130.3.466

Okon-Singer, H., Hendler, T., Pessoa, L., \& Shackman, A. J. (2015). The neurobiology of emotion-cognition interactions: fundamental questions and strategies for future research. Frontiers in Human Neuroscience, 9, 58. https://doi.org/10.3389/fnhum.2015.00058 
Motivation and Emotional Distraction

Okon-Singer, H., Lichtenstein-Vidne, L., \& Cohen, N. (2013). Dynamic modulation of emotional processing. Biological Psychology, 92(3), 480-491. https://doi.org/10.1016/j.biopsycho.2012.05.010

Okon-Singer, H., Tzelgov, J., \& Henik, A. (2007). Distinguishing between automaticity and attention in the processing of emotionally significant stimuli. Emotion, 7(1), 147-157. https://doi.org/10.1037/1528-3542.7.1.147

Oliveira, L., Mocaiber, I., David, I. A., Erthal, F., Volchan, E., \& Pereira, M. (2013). Emotion and attention interaction: a trade-off between stimuli relevance, motivation and individual differences. Frontiers in Human Neuroscience, 7. https://doi.org/10.3389/fnhum.2013.00364

Olofsson, J. K., Nordin, S., Sequeira, H., \& Polich, J. (2008). Affective picture processing: an integrative review of ERP findings. Biological Psychology, 77(3), 247-265. https://doi.org/10.1016/j.biopsycho.2007.11.006

Padmala, S., \& Pessoa, L. (2011). Reward reduces conflict by enhancing attentional control and biasing visual cortical pocessing. Journal of Cognitive Neuroscience, 23(11), 3419-3432. https://doi.org/10.1162/jocn_a_00011

Padmala, S., \& Pessoa, L. (2014). Motivation versus aversive processing during perception. Emotion, 14(3), 450-454. https://doi.org/10.1037/a0036112

Padmala, S., Sambuco, N., Codispoti, M., \& Pessoa, L. (2018). Attentional capture by simultaneous pleasant and unpleasant emotional distractors. Emotion. https://doi.org/10.1037/emo0000401

Padmala, S., Sirbu, M., \& Pessoa, L. (2017). Potential reward reduces the adverse impact of negative distractor stimuli. Social Cognitive and Affective Neuroscience, 12(9), 14021413. https://doi.org/10.1093/scan/nsx067

Parra, M. A., Sánchez, M. G., Valencia, S., \& Trujillo, N. (2018). Attentional bias during emotional processing: evidence from an emotional flanker task using IAPS. Cognition and Emotion, 32(2), 275-285. https://doi.org/10.1080/02699931.2017.1298994

Pavlov, I. P., \& Anrep, G. V. (1928). Conditioned reflexes: an investigation of the physiological activity of the cerebral cortex. London: Oxford University Press.

Pearson, D., Donkin, C., Tran, S. C., Most, S. B., \& Pelley, M. E. L. (2015). Cognitive control and counterproductive oculomotor capture by reward-related stimuli. Visual Cognition, 23(1-2), 41-66. https://doi.org/10.1080/13506285.2014.994252 
Motivation and Emotional Distraction

Peciña, S., \& Berridge, K. C. (2005). Hedonic hot spot in nucleus accumbens shell: Where do $\mu$-opioids cause increased hedonic impact of sweetness? Journal of Neuroscience, 25(50), 11777-11786. https://doi.org/10.1523/JNEUROSCI.2329-05.2005

Pessoa, L. (2005). To what extent are emotional visual stimuli processed without attention and awareness? Current Opinion in Neurobiology, 15(2), 188-196. https://doi.org/10.1016/j.conb.2005.03.002

Pessoa, L. (2008). On the relationship between emotion and cognition. Nature Reviews Neuroscience, 9(2), 148-158. https://doi.org/10.1038/nrn2317

Pessoa, L. (2009). How do emotion and motivation direct executive control? Trends in Cognitive Sciences, 13(4), 160-166. https://doi.org/10.1016/j.tics.2009.01.006

Pessoa, L. (2015). Multiple influences of reward on perception and attention. Visual Cognition, 23(1-2), 272-290. https://doi.org/10.1080/13506285.2014.974729

Pessoa, L., \& Adolphs, R. (2010). Emotion processing and the amygdala: from a 'low road' to 'many roads' of evaluating biological significance. Nature Reviews. Neuroscience, 11(11), 773-783. https://doi.org/10.1038/nrn2920

Pessoa, L., \& Engelmann, J. B. (2010). Embedding reward signals into perception and cognition. Frontiers in Neuroscience, 4. https://doi.org/10.3389/fnins.2010.00017

Pessoa, L., Kastner, S., \& Ungerleider, L. G. (2002). Attentional control of the processing of neutral and emotional stimuli. Cognitive Brain Research, 15(1), 31-45. https://doi.org/10.1016/S0926-6410(02)00214-8

Pessoa, L., \& Ungerleider, L. G. (2004). Neuroimaging studies of attention and the processing of emotion-laden stimuli. Progress in Brain Research, 144, 171-182. https://doi.org/10.1016/S0079-6123(03)14412-3

Phelps, E. A. (2006). Emotion and cognition: insights from studies of the human amygdala. Annual Review of Psychology, 57(1), 27-53. https://doi.org/10.1146/annurev.psych.56.091103.070234

Pool, E., Brosch, T., Delplanque, S., \& Sander, D. (2016). Attentional bias for positive emotional stimuli: a meta-analytic investigation. Psychological Bulletin, 142(1), 79106. https://doi.org/10.1037/bu10000026

Posner, J., Russell, J. A., \& Peterson, B. S. (2005). The circumplex model of affect: An integrative approach to affective neuroscience, cognitive development, and psychopathology. Development and Psychopathology, 17(3), 715-734. https://doi.org/10.1017/S0954579405050340 
Motivation and Emotional Distraction

Posner, M. I., \& Cohen, Y. (1984). Components of visual orienting. Attention and Performance $X, 32,531-556$.

Posner, M. I. (1980). Orienting of attention. Quarterly Journal of Experimental Psychology, 32(1), 3-25. https://doi.org/10.1080/00335558008248231

Posner, M. I. (2016). Orienting of attention: Then and now. Quarterly Journal of Experimental Psychology, 69(10), 1864-1875. https://doi.org/10.1080/17470218.2014.937446

Posner, M. I., \& Snyder, C. R. R. (1975). Attention and cognitive control. In R. L. Solso (Ed.), Information Processing and Cognition: The Loyola Symposium. Lawrence Erlbaum.

Pourtois, G., Grandjean, D., Sander, D., \& Vuilleumier, P. (2004). Electrophysiological correlates of rapid spatial orienting towards fearful faces. Cerebral Cortex, 14(6), 619-633. https://doi.org/10.1093/cercor/bhh023

Pourtois, G., Schettino, A., \& Vuilleumier, P. (2013). Brain mechanisms for emotional influences on perception and attention: what is magic and what is not. Biological Psychology, 92(3), 492-512. https://doi.org/10.1016/j.biopsycho.2012.02.007

Psychology Software Tools, Inc. [E-Prime 2.0]. (2012). Retrieved from http://www.pstnet.com.

Psychology Software Tools, Inc. [E-Prime 3.0]. (2016). Retrieved from http://www.pstnet.com.

Rajkowski, J., Majczynski, H., Clayton, E., \& Aston-Jones, G. (2004). Activation of monkey locus coeruleus neurons varies with difficulty and performance in a target detection task. Journal of Neurophysiology, 92(1), 361-371.

https://doi.org/10.1152/jn.00673.2003

Ramsay, J., Hooker, G., \& Graves, S. (2009). Introduction to Functional Data Analysis. In J. Ramsay, G. Hooker, \& S. Graves, Functional Data Analysis with R and MATLAB (pp. 1-19). New York, NY: Springer New York. https://doi.org/10.1007/978-0-38798185-7_1

Ramsay, J. O., \& Silverman, B. W. (1997). Functional Data Analysis. New York: SpringerVerlag.

Ramsay, J. O., \& Silverman, B. W. (2005). Springer series in statistics. In Functional data analysis. Springer. 
Motivation and Emotional Distraction

Ranganath, C., \& Rainer, G. (2003). Cognitive neuroscience: neural mechanisms for detecting and remembering novel events. Nature Reviews Neuroscience, 4(3), 193202. https://doi.org/10.1038/nrn1052

Ravizza, S. M., \& Delgado, M. R. (2014). Motivational enhancement of cognitive control depends on depressive symptoms. Emotion, 14(4), 646-650.

https://doi.org/10.1037/a0036754

Ravizza, S. M., Goudreau, J., Delgado, M. R., \& Ruiz, S. (2012). Executive function in Parkinson's disease: contributions of the dorsal frontostriatal pathways to action and motivation. Cognitive, Affective, \& Behavioral Neuroscience, 12(1), 193-206. https://doi.org/10.3758/s13415-011-0066-6

Raymond, J. E., Shapiro, K. L., \& Arnell, K. M. (1992). Temporary suppression of visual processing in an rsvp task: an attentional blink? Journal of Experimental Psychology: Human Perception and Performance, 18(3), 849-860.

Reynolds, J. H., Chelazzi, L., \& Desimone, R. (1999). Competitive mechanisms subserve attention in macaque areas V2 and V4. Journal of Neuroscience, 19(5), 1736-1753. https://doi.org/10.1523/JNEUROSCI.19-05-01736.1999

Richter, G., Raban, D. R., \& Rafaeli, S. (2015). Studying gamification: the effect of rewards and incentives on motivation. In T. Reiners \& L. C. Wood (Eds.), Gamification in Education and Business (pp. 21-46). Cham: Springer International Publishing. https://doi.org/10.1007/978-3-319-10208-5_2

Rolls, E. T. (2000). On The brain and emotion. Behavioral and Brain Sciences, 23(2), 219 228. https://doi.org/10.1017/S0140525X00512424

Rondeel, E. W. M., van Steenbergen, H., Holland, R. W., \& van Knippenberg, A. (2015). A closer look at cognitive control: differences in resource allocation during updating, inhibition and switching as revealed by pupillometry. Frontiers in Human Neuroscience, 9. https://doi.org/10.3389/fnhum.2015.00494

Ryan, F. (2002). Detected, selected, and sometimes neglected: cognitive processing of cues in addiction. Experimental and Clinical Psychopharmacology, 10(2), 67-76. https://doi.org/10.1037//1064-1297.10.2.67

Sabatinelli, D., Bradley, M. M., Fitzsimmons, J. R., \& Lang, P. J. (2005). Parallel amygdala and inferotemporal activation reflect emotional intensity and fear relevance. NeuroImage, 24(4), 1265-1270. https://doi.org/10.1016/j.neuroimage.2004.12.015

Sabatinelli, D., Bradley, M. M., Lang, P. J., Costa, V. D., \& Versace, F. (2007). Pleasure rather than salience activates human nucleus accumbens and medial prefrontal cortex. 
Motivation and Emotional Distraction

Journal of Neurophysiology, 98(3), 1374-1379.

https://doi.org/10.1152/jn.00230.2007

Samuels, E., \& Szabadi, E. (2008). Functional neuroanatomy of the noradrenergic locus coeruleus: its roles in the regulation of arousal and autonomic function Part I: Principles of functional organisation. Current Neuropharmacology, 6(3), 235-253. https://doi.org/10.2174/157015908785777229

Sara, S. J. (2009). The locus coeruleus and noradrenergic modulation of cognition. Nature Reviews Neuroscience, 10(3), 211-223. https://doi.org/10.1038/nrn2573

Sawaki, R., \& Luck, S. J. (2010). Capture versus suppression of attention by salient singletons: electrophysiological evidence for an automatic attend-to-me signal. Attention, Perception, \& Psychophysics, 72(6), 1455-1470. https://doi.org/10.3758/APP.72.6.1455

Sawaki, R., Luck, S. J., \& Raymond, J. E. (2015). How attention changes in response to incentives. Journal of Cognitive Neuroscience, 27(11), 2229-2239. https://doi.org/10.1162/jocn_a_00847

Schevernels, H., Bombeke, K., Van der Borght, L., Hopf, J.-M., Krebs, R. M., \& Boehler, C. N. (2015). Electrophysiological evidence for the involvement of proactive and reactive control in a rewarded stop-signal task. NeuroImage, 121, 115-125. https://doi.org/10.1016/j.neuroimage.2015.07.023

Schmidt, L. J., Belopolsky, A. V., \& Theeuwes, J. (2015). Attentional capture by signals of threat. Cognition and Emotion, 29(4), 687-694. https://doi.org/10.1080/02699931.2014.924484

Schultz, W. (2004). Neural coding of basic reward terms of animal learning theory, game theory, microeconomics and behavioural ecology. Current Opinion in Neurobiology, 14(2), 139-147. https://doi.org/10.1016/j.conb.2004.03.017

Schultz, W. (2015). Neuronal reward and decision signals: from theories to data. Physiological Reviews, 95(3), 853-951. https://doi.org/10.1152/physrev.00023.2014

Schupp, H. T., Cuthbert, B. N., Bradley, M. M., Cacioppo, J. T., Ito, T., \& Lang, P. J. (2000). Affective picture processing: the late positive potential is modulated by motivational relevance. Psychophysiology, 37(2), 257-261. https://doi.org/10.1111/14698986.3720257

Schupp, H. T., Flaisch, T., Stockburger, J., \& Junghöfer, M. (2006). Emotion and attention: event-related brain potential studies. Progress in Brain Research, 156, 31-51. https://doi.org/10.1016/S0079-6123(06)56002-9 
Motivation and Emotional Distraction

Serences, J. T., Shomstein, S., Leber, A. B., Golay, X., Egeth, H. E., \& Yantis, S. (2005). Coordination of voluntary and stimulus-driven attentional control in human cortex. Psychological Science, 16(2), 114-122. https://doi.org/10.1111/j.09567976.2005.00791.x

Serences, J. T., Yantis, S., Culberson, A., \& Awh, E. (2004). Preparatory activity in visual cortex indexes distractor suppression during covert spatial orienting. Journal of Neurophysiology, 92(6), 3538-3545. https://doi.org/10.1152/jn.00435.2004

Shafer, A. T., Matveychuk, D., Penney, T., O’Hare, A. J., Stokes, J., \& Dolcos, F. (2012). Processing of emotional distraction Is both automatic and modulated by attention: evidence from an event-related fMRI investigation. Journal of Cognitive Neuroscience, 24(5), 1233-1252. https://doi.org/10.1162/jocn_a_00206

Shen, Y. J., \& Chun, M. M. (2011). Increases in rewards promote flexible behavior. Attention, Perception, \& Psychophysics, 73(3), 938-952. https://doi.org/10.3758/s13414-010-0065-7

Shenhav, A., Botvinick, M. M., \& Cohen, J. D. (2013). The expected value of control: an integrative theory of anterior cingulate cortex function. Neuron, 79(2), 217-240. https://doi.org/10.1016/j.neuron.2013.07.007

Shenhav, A., Musslick, S., Lieder, F., Kool, W., Griffiths, T. L., Cohen, J. D., \& Botvinick, M. M. (2017). Toward a rational and mechanistic account of mental effort. Annual Review of Neuroscience, 40(1), 99-124. https://doi.org/10.1146/annurev-neuro072116-031526

Shiffrin, R. M., \& Schneider, W. (1977). Controlled and automatic human information processing: II. Perceptual learning, automatic attending and a general theory. Psychological Review, 84(2), 127-190. https://doi.org/10.1037/0033-295X.84.2.127

Siegrist, M. (1995). Effects of taboo words on color-naming performance on a Stroop test. Perceptual and Motor Skills, 81(3_supp1), 1119-1122. https://doi.org/10.2466/pms.1995.81.3f.1119

Sirois, S., \& Brisson, J. (2014). Pupillometry: pupillometry. Wiley Interdisciplinary Reviews: Cognitive Science, 5(6), 679-692. https://doi.org/10.1002/wcs.1323

Small, D. M., Gitelman, D., Simmons, K., Bloise, S. M., Parrish, T., \& Mesulam, M.-M. (2005). Monetary incentives enhance processing in brain regions mediating top-down control of attention. Cerebral Cortex (New York, N.Y.: 1991), 15(12), 1855-1865. https://doi.org/10.1093/cercor/bhi063 
Motivation and Emotional Distraction

Snowden, R. J., O’Farrell, K. R., Burley, D., Erichsen, J. T., Newton, N. V., \& Gray, N. S. (2016). The pupil's response to affective pictures: role of image duration, habituation, and viewing mode: pupil response to affective pictures. Psychophysiology, 53(8), 1217-1223. https://doi.org/10.1111/psyp.12668

Steenbergen, H. van, Band, G. P. H., \& Hommel, B. (2009). Reward counteracts conflict adaptation: evidence for a role of affect in executive control. Psychological Science, 20(12), 1473-1477. https://doi.org/10.1111/j.1467-9280.2009.02470.x

Stroop, J. R. (1935). Studies of interference in serial verbal reactions. Journal of Experimental Psychology, 18(6), 643-662. https://doi.org/10.1037/h0054651

Stürmer, B. (2011). Reward and punishment effects on error processing and conflict control. Frontiers in Psychology, 2. https://doi.org/10.3389/fpsyg.2011.00335

Surguladze, S. A., Brammer, M. J., Young, A. W., Andrew, C., Travis, M. J., Williams, S. C. R., \& Phillips, M. L. (2003). A preferential increase in the extrastriate response to signals of danger. NeuroImage, 19(4), 1317-1328. https://doi.org/10.1016/S10538119(03)00085-5

Sussman, T. J., Jin, J., \& Mohanty, A. (2016). Top-down and bottom-up factors in threatrelated perception and attention in anxiety. Biological Psychology, 121, 160-172. https://doi.org/10.1016/j.biopsycho.2016.08.006

Theeuwes, J. (1994). Stimulus-driven capture and attentional set: selective search for color and visual abrupt onsets. Journal of Experimental Psychology. Human Perception and Performance, 20(4), 799-806. https://doi.org/10.1037/0096-1523.20.4.799

Theeuwes, J., Atchley, P., \& Kramer, A.F. (2000). On the time course of top-down and bottom-up control of visual attention. In Attention and Performance. MIT Press. Retrieved from https://research.vu.nl/en/publications/b1402c97-d21e-45eb-8f8afcc50bb7e11e

Theeuwes, J. (1991). Exogenous and endogenous control of attention: the effect of visual onsets and offsets. Perception \& Psychophysics, 49(1), 83-90. https://doi.org/10.3758/BF03211619

Theeuwes, J. (2010). Top-down and bottom-up control of visual selection. Acta Psychologica, 135(2), 77-99. https://doi.org/10.1016/j.actpsy.2010.02.006

Theeuwes, J., de Vries, G. J., \& Godijn, R. (2003). Attentional and oculomotor capture with static singletons. Perception \& Psychophysics, 65(5), 735-746. https://doi.org/10.3758/BF03194810 
Motivation and Emotional Distraction

Thorndike, E. L. (1927). The Law of Effect. The American Journal of Psychology, 39(1/4), 212-222. https://doi.org/10.2307/1415413

van den Berg, B., Krebs, R. M., Lorist, M. M., \& Woldorff, M. G. (2014). Utilization of reward-prospect enhances preparatory attention and reduces stimulus conflict. Cognitive, Affective \& Behavioral Neuroscience, 14(2), 561-577. https://doi.org/10.3758/s13415-014-0281-z

Van der Stigchel, S., \& Theeuwes, J. (2006). Our eyes deviate away from a location where a distractor is expected to appear. Experimental Brain Research, 169(3), 338-349. https://doi.org/10.1007/s00221-005-0147-2

van der Wel, P., \& van Steenbergen, H. (2018). Pupil dilation as an index of effort in cognitive control tasks: a review. Psychonomic Bulletin \& Review, 25(6), 2005-2015. https://doi.org/10.3758/s13423-018-1432-y

Van Dillen, L. F., Heslenfeld, D. J., \& Koole, S. L. (2009). Tuning down the emotional brain: An fMRI study of the effects of cognitive load on the processing of affective images. NeuroImage, 45(4), 1212-1219. https://doi.org/10.1016/j.neuroimage.2009.01.016 Vanderhasselt, M.-A., Remue, J., Ng, K. K., \& De Raedt, R. (2014). The interplay between the anticipation and subsequent online processing of emotional stimuli as measured by pupillary dilatation: the role of cognitive reappraisal. Frontiers in Psychology, 5. https://doi.org/10.3389/fpsyg.2014.00207

Vogt, J., De Houwer, J., Koster, E. H. W., Van Damme, S., \& Crombez, G. (2008). Allocation of spatial attention to emotional stimuli depends upon arousal and not valence. Emotion, 8(6), 880-885. https://doi.org/10.1037/a0013981

Vogt, J., Lozo, L., Koster, E. H. W., \& Houwer, J. D. (2011). On the role of goal relevance in emotional attention: disgust evokes early attention to cleanliness. Cognition and Emotion, 25(3), 466-477. https://doi.org/10.1080/02699931.2010.532613

Vuilleumier, P., Armony, J. L., Driver, J., \& Dolan, R. J. (2001). Effects of attention and emotion on face processing in the human brain: an event-related fMRI study. Neuron, 30(3), 829-841. https://doi.org/10.1016/S0896-6273(01)00328-2

Vuilleumier, P. (2005). How brains beware: neural mechanisms of emotional attention. Trends in Cognitive Sciences, 9(12), 585-594. https://doi.org/10.1016/j.tics.2005.10.011

Vuilleumier, P. (2015). Affective and motivational control of vision. Current Opinion in Neurology, 28(1), 29. https://doi.org/10.1097/WCO.0000000000000159 
Motivation and Emotional Distraction

Vuilleumier, P., Richardson, M. P., Armony, J. L., Driver, J., \& Dolan, R. J. (2004). Distant influences of amygdala lesion on visual cortical activation during emotional face processing. Nature Neuroscience, 7(11), 1271-1278. https://doi.org/10.1038/nn1341

Walsh, A. T., Zhao, J., Grimshaw, G. M., \& Most, S. (in prep). The influence of warnings on proactive control of spatial and temporal emotional distraction.

Watson, P., Pearson, D., Wiers, R. W., \& Le Pelley, M. E. (2019). Prioritizing pleasure and pain: attentional capture by reward-related and punishment-related stimuli. Current Opinion in Behavioral Sciences, 26, 107-113. https://doi.org/10.1016/j.cobeha.2018.12.002

Watts, F. N., McKenna, F. P., Sharrock, R., \& Trezise, L. (1986). Colour naming of phobiarelated words. British Journal of Psychology, 77(1), 97-108. https://doi.org/10.1111/j.2044-8295.1986.tb01985.x

Wiens, S., Sand, A., \& Olofsson, J. K. (2011). Nonemotional features suppress early and enhance late emotional electrocortical responses to negative pictures. Biological Psychology, 86(1), 83-89. https://doi.org/10.1016/j.biopsycho.2010.11.001

Willenbockel, V., Sadr, J., Fiset, D., Horne, G. O., Gosselin, F., \& Tanaka, J. W. (2010). Controlling low-level image properties: The SHINE toolbox. Behavior Research Methods, 42(3), 671-684. https://doi.org/10.3758/BRM.42.3.671

Wolfe, J. M., \& Horowitz, T. S. (2004). What attributes guide the deployment of visual attention and how do they do it? Nature Reviews Neuroscience, 5(6), 495-501. https://doi.org/10.1038/nrn1411

Yamaguchi, M., \& Nishimura, A. (2018). Modulating proactive cognitive control by reward: differential anticipatory effects of performance-contingent and non-contingent rewards. Psychological Research, 1-17. https://doi.org/10.1007/s00426-018-1027-2

Yantis, S., \& Jonides, J. (1984). Abrupt visual onsets and selective attention: Evidence from visual search. Journal of Experimental Psychology: Human Perception and Performance, 10(5), 601-621. https://doi.org/10.1037/0096-1523.10.5.601

Yates, A., Ashwin, C., \& Fox, E. (2010). Does emotion processing require attention? The effects of fear conditioning and perceptual lpad. Emotion (Washington, D.C.), 10(6), 822-830. https://doi.org/10.1037/a0020325

Yee, D. M., \& Braver, T. S. (2018). Interactions of motivation and cognitive control. Current Opinion in Behavioral Sciences, 19, 83-90. https://doi.org/10.1016/j.cobeha.2017.11.009 
Motivation and Emotional Distraction

Yiend, J. (2010). The effects of emotion on attention: a review of attentional processing of emotional information. Cognition and Emotion, 24(1), 3-47. https://doi.org/10.1080/02699930903205698

Yokum, S., Ng, J., \& Stice, E. (2011). Attentional bias to food images associated with elevated weight and future weight gain: An fMRI Study. Obesity, 19(9), 1775-1783. https://doi.org/10.1038/oby.2011.168

Zhang, J., Berridge, K. C., Tindell, A. J., Smith, K. S., \& Aldridge, J. W. (2009). A neural computational model of incentive salience. PLOS Computational Biology, 5(7), e1000437. https://doi.org/10.1371/journal.pcbi.1000437 
Motivation and Emotional Distraction

\section{Appendices Contents}

Page

Appendix A. Preprint of the publication for Experiment 1 160

Appendix B. Table S1. IAPS image numbers for each image set 176

Appendix C. Preprint of publication for Experiment 2 177

Appendix D. Open Science Framework preregistration for Experiment 2 215

Appendix E. These figures contain the output of the functional $t$-tests 230

Appendix F. As Predicted preregistration for Experiment 4 234

Appendix G. Supplementary analysis reward criterion 236

Appendix H. Analysis of the influence of image repetition in Experiment 1 237 
Motivation and Emotional Distraction

Appendix A. Preprint of the publication for Chapter 2

Walsh, A. T., Carmel, D., Harper, D., \& Grimshaw, G. M. (2018). Motivation enhances control of positive and negative emotional distractions. Psychonomic Bulletin \& Review, 25(4), 1556-1562.

\title{
Motivation enhances control of positive and negative emotional distractions
}

\author{
Amy T. Walsh ${ }^{1}$, David Carmel ${ }^{2}$, David Harper ${ }^{1}$ and Gina M. Grimshaw ${ }^{1}$ \\ ${ }^{1}$ School of Psychology, Victoria University of Wellington, New Zealand \\ ${ }^{2}$ Department of Psychology, University of Edinburgh, UK
}

Address Correspondence to: Gina Grimshaw, School of Psychology, Victoria University of Wellington, PO Box 600, Wellington 6140, New Zealand. gina.grimshaw@vuw.ac.nz;

Other authors: Amy Walsh, School of Psychology, Victoria University of Wellington, PO Box 600, Wellington 6140, New Zealand, amy.walsh@vuw.ac.nz, +64 04463 6420; David Carmel, Psychology Department, University of Edinburgh, 7 George Square, Edinburgh EH8 9JZ, UK; dave.carmel@ed.ac.uk,+44-131-6503455; David Harper, School of Psychology, Victoria University of Wellington, PO Box 600, Wellington 6140, New Zealand, david.harper@vuw.ac.nz, +64 044635561.

Research was supported by a grant from the Royal Society of New Zealand Marsden Fund (VUW1307) to Gina Grimshaw and David Carmel. Findings were reported at the meeting of the Australasian Cognitive Neuroscience Society, November 2016. We thank Al Abenoja for programming assistance. Materials, data and analyses are available at osf.io/qu2d6.

Disclosure of interest statement: The authors report no conflict of interest.

This manuscript was accepted for publication in Psychonomic Bulletin and Review on 4/12/2017. 
Motivation and Emotional Distraction

Using cognitive control to ignore distractions is essential for successfully achieving our goals. In emotionallyneutral contexts, motivation can reduce interference from irrelevant stimuli by enhancing cognitive control. However, attention is commonly biased towards emotional stimuli, making them potent distractors. Can motivation aid control of emotional distractions, and does it do so similarly for positive and negative stimuli? Here, we examined how task motivation influences control of distraction from positive, negative, and neutral scenes. Participants completed a simple perceptual task while attempting to ignore task-irrelevant images. One group received monetary reward for fast and accurate task performance; another (control) group did not.

Overall, both negative (mutilation) and positive (erotic) images caused greater slowing of responses than neutral images of people, but emotional distraction was reduced with reward. Crucially, despite the different motivational directions associated with negative and positive stimuli, reward reduced negative and positive distraction equally. Our findings suggest that motivation may encourage the use of a sustained proactive control strategy that can effectively reduce the impact of emotional distraction.

Keywords: emotion, motivation, distraction, cognitive control, reward 


\section{Motivation and Emotional Distraction}

Of the many sensory inputs competing for our attention, emotional content is prioritised (Pourtois, Schettino, \& Vuilleumier, 2013). Although attending to goal-irrelevant emotional information is often adaptive, it can sometimes be detrimental (Okon-singer, Tzelgov, \& Henik, 2007). From the basketball player who ignores a heckling crowd to make a freethrow, to the ex-smoker who ignores smoking-related cues, we all suppress emotional distractors to achieve our goals. To what extent can we draw on motivation when attempting to control emotional distractions? Does motivation to win the game, or improve health, increase the ability to ignore these powerful emotional cues?

Ignoring distractors is an important aspect of cognitive control (Geng, 2014). A growing literature indicates that motivation facilitates the effective use of control in emotionally-neutral contexts (Chiew \& Braver, 2013), reducing interference from irrelevant distractors when rewards are offered (Botvinick \& Braver, 2015; Padmala \& Pessoa, 2011). But emotional stimuli are potent distractors, in part due to their biological relevance. Prioritisation of emotional stimuli may occur via separate mechanisms from classic stimulusdriven and goal-driven processes of attentional selection (Pourtois et al., 2013). Additionally, processing emotional content may use capacity-limited resources that are also recruited for cognitive control (Pessoa, 2009); if the resources required to ignore emotional distractors are partly consumed by attending to their content, they may be especially difficult to ignore. Given these differences in processing of emotional and neutral stimuli, it is unclear whether emotional distraction can be attenuated by reward at all.

Findings from two recent studies (Padmala \& Pessoa, 2014; Padmala, Sirbu, \& Pessoa, 2017) suggest that motivation can increase the ability to ignore emotionally-negative images. Participants judged the orientation of lines flanking a centrally-presented distractor image. Pre-cues indicated whether reward was available on each trial. Negative images slowed response times (RTs) relative to neutral images; but on reward trials, this slowing was eliminated. Further support comes from an event-related-potential study (Wei, Wang, \& Ji, 2016) in which participants responded to the colour of negative and neutral words. The emotional modulation of the P3 component was eliminated on cued-incentive trials. Thus, motivation can modulate neural and behavioural responses to irrelevant emotionally-negative stimuli. Whether motivation can reduce distraction from emotionally-positive stimuli, however, remains unknown. 
Motivation and Emotional Distraction

Biologically-relevant, high arousal, positive images can attract attention just as powerfully as negative images (Grimshaw, Kranz, Carmel, Moody, \& Devue, 2017; Gupta, Hur, \& Lavie, 2016; Most, Smith, Cooter, Levy, \& Zald, 2007), and in some contexts they can be even more difficult to ignore. For example, using a letter-search task with centrallypresented task-irrelevant emotional images, Gupta and colleagues (2016) found that increasing the task's perceptual load eliminates distraction from negative but not positive images. Similarly, Most and colleagues (2007) report that pre-cueing the identity of a nonemotional target in a stream of rapidly-presented images reduces distraction from negative but not positive images. If motivation acts via similar control mechanisms to those elicited by these attentional manipulations, then we might expect reward to attenuate negative but not positive distraction. However, conflicting findings have been reported. Grimshaw and colleagues (2017) show that increasing distractor-frequency (also a control manipulation; Bugg \& Crump, 2012) reduces distraction from both negative and positive images. Thus, whether reward affects negative and positive distraction to a similar degree may depend on the specific control mechanisms that are engaged.

Another consideration is that emotional distractors have motivational value. If reward affects control by increasing the motivational value of task-relevant targets while decreasing the relative value of distractors, it may be less effective against emotional than neutral distractors. Furthermore, the function of emotional stimuli is to prepare the body for action, guiding us to approach or withdraw (Rolls, 2005). Reward might therefore influence control of positive and negative distractors differently, due to their opposing motivational directions.

To investigate whether motivation can enhance control of emotional distraction, and if so, whether it does so similarly for positive and negative distractors, we compared the effect of reward on distraction by equally arousing positive and negative images. Participants searched for a target-letter within an array that appeared above and below a centrallypresented irrelevant image (intact on $25 \%$ of trials, scrambled on the rest). Low frequency of intact-distractors elicits relatively poor control of distraction (Bugg \& Crump, 2012; Grimshaw et al., 2017). Half the participants received monetary-reward for fast and accurate performance. We expected reward to reduce distraction overall, but were specifically interested in whether reward would attenuate distraction from emotional images as effectively as it does for neutral ones. We further compared the effect of reward on distraction from negative versus positive images. 
Motivation and Emotional Distraction

\section{Method}

\section{Participants}

Seventy-three participants (women only, to reduce within-group variance, and to allow for use of images that were carefully calibrated for valence and arousal ratings, which differ across genders) were randomly assigned to the control $(n=36)$ and reward $(n=37)$ groups. One outlier participant from the reward group was excluded from analysis and replaced (see Sample Size Determination), giving 36 participants per group (mean age 22.40; 18-37 years). All participants spoke fluent English, had normal or corrected-to-normal vision and reported no current treatment for depression or anxiety. Analyses were undertaken after all data were collected. The study was approved by the Victoria University of Wellington Human Ethics Committee.

\section{Stimuli and Apparatus}

Participants ran in groups of one to four in a dimly-lit room in separate booths. The experiment was run using E-Prime 2.0 (Psychology Software Tools, Pittsburgh, PA) on DellPrecision-T1700 personal-computers with 24" monitors at 1920x1080-pixel resolution and a $120 \mathrm{~Hz}$ refresh rate. A chin-rest maintained a viewing distance of $57 \mathrm{~cm}$.

Twelve images for each category ${ }^{1}$ (negative, neutral, positive) were drawn from the International Affective Picture System (IAPS; Lang, Bradley, \& Cuthbert, 2008). The positive (erotic couples) and negative (mutilation) images were matched on arousal using normative ratings from women (arousal: positive $M=6.31$, negative $M=6.53$; valence: negative $M=1.64$, positive $M=6.23$ ), to avoid confounding valence differences with arousal. The neutral images contained people and thus had biological and social relevance (like emotional images), but lacked emotional valence $(M=5.01)$ and arousal $(M=3.07)$. To create scrambles, images were divided into 36 x 36 segments that were randomly recombined in PhotoScape-v3.7. Scrambled images thus had the same lower level visual properties as intact distractors; comparing performance on intact vs. scrambled trials therefore estimates distraction due to the images' meaning. All images were matched for luminance and contrast using the SHINE-MATLAB-toolbox (Willenbockel et al., 2010). 
Motivation and Emotional Distraction

\section{Design and Procedure}

Figure 1A illustrates the distraction task. Each trial began with a central white fixation cross (between 417-833 ms) on a black background, followed by a $200 \mathrm{~ms}$ target display, which comprised a centrally-presented colour image $\left(11^{\circ}\right.$ width $x 8.26^{\circ}$ height; intact on $25 \%$ of trials, scrambled on $75 \%$ ), flanked by six white letters $\left(0.86^{\circ}\right.$ x $\left.0.92^{\circ}\right)$; three located $0.75^{\circ}$ above and three $0.75^{\circ}$ below the image's horizontal edge. Five of the letters were 'O's and the target was ' $\mathrm{K}$ ' or ' $\mathrm{N}$ '. Participants were instructed to ignore the images and respond quickly and accurately to indicate whether ' $\mathrm{K}$ ' or ' $\mathrm{N}$ ' was present, using keys ' 1 ' and ' 2 ' (counterbalanced across participants) on the number-pad. Participants responded during an $1800 \mathrm{~ms}$ response-window from stimulus-offset (anticipatory responses $<200 \mathrm{~ms}$ were excluded; $<0.01 \%$ of trials). Failure to respond was recorded as an error. A $600 \mathrm{~ms}$ blank screen followed the response, followed by visual feedback (100 ms): 'correct', 'incorrect', or 'please respond faster' (if no response was made). A random inter-trial-interval ranged between 207-623 ms. Target letter and location were counterbalanced across trials and trial order was randomised.

Participants completed 24 practice trials (six intact, 18 scrambled) and a baselineblock of 48 trials (12 intact, 36 scrambled); these blocks contained neutral images, different from those presented on experimental trials. The experiment was split into two halves (superblocks) of 192 trials (counterbalanced). One super-block contained negative and neutral distractors; the other contained positive and neutral distractors (separated by a three-minute break to limit valence carry-over effects). Each super-block comprised two emotional and two neutral blocks (separated by self-timed breaks), presented in ABBA (counterbalanced) order. Within a block (48 trials; 12 intact, 36 scrambled), all images were either negative, neutral, or positive, mixed with scrambles created from the same images. Each intact image was presented once in a block, although images were repeated (emotional images twice; neutral images four times) across the experiment to increase trial numbers. This yielded four possible block orders, counterbalanced across participants.

\section{Reward Condition.}

After the baseline-block, participants were informed about the potential to earn one point pertrial if they were correct and faster than their median RT from the baseline-block. Success was indicated by a pleasant sound during the $600 \mathrm{~ms}$ post-response-window. Winning points 
Motivation and Emotional Distraction

led to achieving 'levels'; reaching a new level increased earnings by $\$ 2.50$. Participants began on level 1 (\$10). Level 2 required 91 points $(\$ 12.50)$. Ninety-one additional points were needed to ascend to each new level, up to level 5 (\$20). During each break, point total and current level appeared above an animated coin display, with mean RT and percent accuracy for the preceding block. Participants were reminded to be fast and accurate. A warning was provided if accuracy fell below 95\%. Participants earned between $\$ 15$ and $\$ 20$ $(M=\$ 16.22)$.

\section{Control Condition.}

Control participants completed the task as above, but were offered $\$ 15$, unconnected to performance. They received visual but not auditory feedback. During the breaks instead of performance feedback and the coin animation; they saw 'please wait...', and instructions to take a break.

\section{Sample Size Determination}

To determine the sample size needed to detect an effect of reward on emotional distraction, we used the effect size $\left(d_{s}=.84\right)$ from Grimshaw et al. (2017), using distractor-frequency $(25 \%$ vs. $75 \%)$ as a proxy for the potential effect of reward on emotional distraction (RTintact - RT-scrambled, collapsed across positive and negative). At $90 \%$ power $(\alpha=.05)$, this yielded 26 participants per group. We rounded up to 36 participants per group for counterbalancing purposes. One participant from the reward group was excluded from analyses and replaced because she showed $466 \mathrm{~ms}$ of negative distraction, $>4$ SDs above the mean for negative distraction in the reward condition.

\section{Results}

RTs and accuracy for neutral blocks did not differ across the two super-blocks (intact and scrambled conditions; $p$ 's $>.260$ ), so we collapsed the neutral blocks in analyses. Mean correct RTs and accuracy for each condition, and comparisons between intact and scrambled conditions, are presented in Table 1. Degrees of freedom are adjusted for heterogeneity of variance (Greenhouse-Geisser) where necessary. Effect sizes are Hedge's $g$ for betweensubject comparisons, and Cohen's $d_{r m}$ for within-subject comparisons (Lakens, 2013). Confidence-intervals are $95 \%$. 
Motivation and Emotional Distraction

RTs

See Figure 1B for mean distraction in each condition. Correct RTs were analysed in a 2 (reward: reward, control) x 3 (distractor-valence: negative, neutral, positive) x 2 (distractortype: intact, scrambled) analysis of variance (ANOVA), with reward as the between-subjects factor and the other factors within-subjects.

Main effects of distractor-valence $F(1.85,129.20)=11.01, p<.001, \eta_{p}{ }^{2}=.14$ and distractor-type $F(1,70)=122.36, p<.001, \eta_{p}{ }^{2}=.64$, were qualified by a distractor-valence $\mathrm{x}$ distractor-type interaction $F(2,140)=30.94, p<.001, \eta_{p}{ }^{2}=.31$. Comparing distraction (intact-RT-scrambled-RT) showed that the interaction was due to greater negative and positive than neutral distraction: negative distraction $(M=75 \mathrm{~ms}, S D=70 \mathrm{~ms})$ was greater than neutral distraction $(M=26 \mathrm{~ms}, S D=22 \mathrm{~ms}), t(71)=6.73, p<.001, d_{r m}=.80$, CI [35, 63]; and positive distraction $(M=65 \mathrm{~ms}, S D=64 \mathrm{~ms})$ was greater than neutral distraction, $t(71)=5.63, p<.001, d_{r m}=.73 \mathrm{CI}[25,53]$. Positive and negative distraction did not significantly differ, $t(71)=1.68, p=.098, d_{r m}=.15$, CI [-2, 22].

A main effect of reward confirmed that the reward group $(M=571 \mathrm{~ms}, S D=89 \mathrm{~ms})$ was faster than the control group $(M=654 \mathrm{~ms}, S D=126 \mathrm{~ms}), F(1,70)=10.40, p=.002, \eta_{p}{ }^{2}$ $=.13$. The predicted reward $\mathrm{x}$ distractor-type interaction, $F(1,70)=10.14, p=.002, \eta_{p}{ }^{2}=.13$, was followed-up by comparing distraction between the groups. The reward group was $32 \mathrm{~ms}$ less distracted $(M=39 \mathrm{~ms}, S D=34 \mathrm{~ms})$ than the control group $(M=71 \mathrm{~ms}, S D=49 \mathrm{~ms})$, $t(70)=3.18, p=.002, g=.74$, CI $[12,52]$.

A reward $\mathrm{x}$ distractor-type $\mathrm{x}$ distractor-valence interaction ${ }^{2}, F(2,140)=5.20, p=$ $.007, \eta_{p}{ }^{2}=.07$, was driven by a reduction in emotional distraction with reward: negative distraction was $42 \mathrm{~ms}$ lower in the reward group $(M=54 \mathrm{~ms}, S D=60 \mathrm{~ms})$ compared to the control group $(M=96 \mathrm{~ms}, S D=73 \mathrm{~ms}), t(70)=2.70, p=.009, g=.63$, CI [11, 74]. Positive distraction was $46 \mathrm{~ms}$ lower in the reward group $(M=88 \mathrm{~ms}, S D=70 \mathrm{~ms})$ compared to the control group $(M=42, S D=48 \mathrm{~ms}), t(62.11)=3.24, p=.002, g=.76, \mathrm{CI}[18,74]$. In contrast, for neutral distraction there was no significant difference between the reward $(M=$ $22 \mathrm{~ms}, S D=18 \mathrm{~ms})$ and control $(M=30 \mathrm{~ms}, S D=25 \mathrm{~ms})$ groups, $t(70)=1.46, p=.150, g=$ .34 , CI $[-3,18]$. Emotional distraction is clearly reduced by reward.

To directly test our question of whether positive and negative distraction were affected similarly by reward, we ran an ANOVA with the same factors but excluded neutral 


\section{Motivation and Emotional Distraction}

blocks. The reward $\mathrm{x}$ distractor-type $\mathrm{x}$ distractor-valence interaction was not close to significant $F(1,70)=0.07, p=.788, \eta_{p}{ }^{2}=.001$. An equivalence test (Lakens, 2017), using the smallest effect size we could detect at $90 \%$ power $\left(d_{z}=.39\right)$ as the lower and upper bounds, indicated that the size $\left(d_{z}=0.03\right)$ of the group difference did not differ between positive and negative conditions, $t(35)=2.14, p=.020$; the effect of reward was not influenced by distractor-valence.

No other effects reached significance.

\section{Accuracy}

Accuracy (proportion correct) was similarly analysed in a $2 \times 3 \times 2$ ANOVA. A main effect of reward showed that the reward group $(M=.899, S D=.061)$ was less accurate than the control group $(M=.935, S D=.039), F(1,70)=8.82 p=.004, \eta_{p}^{2}=.11$, indicating a speedaccuracy-trade-off (see Inverse-efficiency, below). Main effects of distractor-valence, $F(2$, $140)=10.74, p<.001, \eta_{p}{ }^{2}=.13$, and distractor-type, $F(1,70)=43.17, p<.001, \eta_{p}{ }^{2}=.38$, were qualified by a marginal distractor-valence $\mathrm{x}$ distractor-type interaction, $F(2,140)=2.51$, $p=.085, \eta_{p}{ }^{2}=.04$. Follow-up tests revealed that negative distraction was marginally higher than neutral distraction, $t(71)=1.79, p=.078, d_{r m}=.30$, CI [-.002, .037]. Positive distraction was significantly higher than neutral distraction, $t(71)=2.17, p=.033, d_{r m}=.36$, CI [.002, $.041]$. Positive and negative distraction did not differ from each other, $t(71)=0.39, p=.700$, $d_{r m}=.06 \mathrm{CI}[-.025, .017]$. RT and accuracy results converge to reflect greater emotional than neutral distraction. No other effects reached significance.

\section{Inverse-efficiency}

To ensure that a speed-accuracy-trade-off did not account for the effect of reward on emotional distraction, we conducted an ANOVA on inverse-efficiency scores (adjusting RTs for accuracy: [RT/proportion correct], see Bruyer \& Brysbaert, 2011). Consistent with the RT analysis, the distractor-type $\mathrm{x}$ reward interaction, $F(1,70)=6.05, p=.016, \eta_{p}{ }^{2}=.08$; and distractor-valence $\mathrm{x}$ distractor-type $\mathrm{x}$ reward interaction, $F(2,140)=4.75, p=.010, \eta_{p}{ }^{2}=.06$, remained significant. Negative distraction, $t(70)=2.29, p=.025, g=.53$, CI $[9,131]$, and positive distraction, $t(70)=2.62, p=.011, g=.61$, CI $[14,106]$, were both attenuated by reward, whereas neutral distraction did not differ between groups, $t(70)=0.06, p=.951, g=$ .01 , CI $[-17,18]$. All other significant effects mirrored those in the RT analyses. 
Motivation and Emotional Distraction

\section{Discussion}

We find that emotional images are more distracting than neutral ones, but that emotional distraction is attenuated by the availability of performance-contingent reward. Only two previous studies have addressed the effect of reward on suppression of negative images (Padmala \& Pessoa, 2014; Padmala et al., 2017). We replicate and extend these findings, showing that despite the opposing motivational directions associated with positive and negative stimuli (approach and withdrawal), motivation is similarly effective in enhancing control of both positive and negative distractions.

The benefit of reward for both negative and positive distraction parallels the effect of increased distractor frequency (Grimshaw et al., 2017). According to the Dual Mechanisms of Control Framework (Braver, Gray, \& Burgess, 2007), both expectations (manipulated by distractor-frequency), and motivation (manipulated by reward), act in a top-down manner to shift control from a reactive strategy (triggered when distraction occurs) to a proactive one (using anticipatory control to prevent distraction before it occurs). The influence of reward on proactive control has been repeatedly demonstrated in emotionally-neutral contexts (see Botvinick \& Braver, 2015). Here, we show that it might be possible to extend this model to contexts in which distractors are negative and positive stimuli. Surprisingly, reward did not attenuate neutral distraction. Neutral distraction was relatively low, suggesting a possible floor-effect, or an effect too small to be detected in this study.

Our finding contrasts reports of other attentional manipulations that attenuate negative but not positive distraction. Notably, these studies manipulated perceptual load (Gupta et al., 2016) and attentional settings (Most et al., 2007) to affect control. These discrepancies logically suggest that multiple control mechanisms may be used to control emotional distractions, depending on the factor that elicits control (e.g., perceptual load vs. reward). We note that proactive and reactive are collective terms (referring to timing) that each comprise multiple possible mechanisms. One possibility is that other (non-reward) manipulations affect reactive control, which could be sensitive to emotional valence. Alternatively, other (nonreward) control manipulations could affect a different proactive mechanism than motivation does. Further studies to directly compare the effects of different manipulations will be useful for identifying the conditions under which different control mechanisms are engaged. Online measures of control (e.g., pupillometry or electrophysiology; e.g., Chiew \& Braver, 2013) will provide insight into the time-course of control elicited by different manipulations. 
Motivation and Emotional Distraction

The present findings suggest a number of future extensions. First, blocking distractors by valence enabled us to maximise emotional distraction while minimizing carry-over effects, so as to demonstrate the effect of reward on positive and negative distraction separately. Blocking allows participants to predict a distractor's valence. To the extent that expectations influence the mechanisms used to control emotional distraction, it is possible that reward might be equally effective for controlling distractions with predictable valence, but not distractors whose valence is unpredictable. Designs in which distractor-valence is unpredictable will be useful in examining how expectations affect control of distraction. Second, our use of between-subjects, blocked incentive (intended to maximise the impact of reward), resulted in a strong test of whether motivation can act globally to affect sustained control of negative and positive distractions. Our manipulation contrasts with that of Padmala and colleagues $(2014 ; 2017)$, who manipulated reward within-subjects, via pre-trial cues. Their findings therefore reflect a dynamic, trial-by-trial shift in control of negative distraction. Future research should address whether these dynamic shifts are possible when controlling positive distractions. Lastly, we focussed on the distracting effect of specific categories of emotional distractors (erotic couples, mutilations); and did so only in women. The present findings invite extension to different categories of images, and to men and women.

We often encounter situations in which positive and negative cues compete with more mundane, but goal-relevant stimuli. Cognitive control mechanisms are necessary in these situations to negotiate an optimal outcome. Our study shows that motivation is one way of enhancing control over both negative and positive distractions. The relationship between emotion, motivation, and cognitive control is complex (Pessoa, 2009), and research examining their interplay is in its infancy. Further exploration of their interactions will provide richer understanding of the conditions under which current goals are successfully achieved. 
Motivation and Emotional Distraction

\section{References}

Botvinick, M., \& Braver, T. (2015). Motivation and cognitive control: from behavior to neural mechanism. Annual Review of Psychology, 66(1), 83-113. doi:10.1146/annurev-psych-010814-015044

Braver, T. S., Gray, J. R., \& Burgess, G. C. (2007). Explaining the many varieties of working memory variation: Dual mechanisms of cognitive control. In A. R. A. Conway, C.

Jarrold, M. J. Kane, A. Miyake \& J. N. Towse (Eds.), Variation in working memory (pp.76-106). Oxford: Oxford University Press.

Bruyer, R., \& Brysbaert, M. (2011). Combining speed and accuracy in cognitive psychology: is the inverse efficiency score (IES) a better dependent variable than the mean reaction time (RT) and the percentage of errors (PE)?. Psychologica Belgica, 51(1), 5-13. doi:10.5334/pb-51-1-5

Bugg, J. M., \& Crump, M. J. (2012). In support of a distinction between voluntary and stimulus-driven control: A review of the literature on proportion congruent effects. Frontiers in Psychology, 3:367. doi:10.3389/fpsyg.2012.00367

Chiew, K. S., \& Braver, T. S. (2013). Temporal dynamics of motivation-cognitive control interactions revealed by high-resolution pupillometry. Frontiers in Psychology, 4:15. doi:10.3389/fpsyg.2013.00015

Geng, J. J. (2014). Attentional mechanisms of distractor suppression. Current Directions in Psychological Science, 23(2), 147-153. doi:10.1177/0963721414525780

Grimshaw, G. M., Kranz, L. S., Carmel, D., Moody, R. E., \& Devue, C. (2017). Contrasting reactive and proactive control of emotional distraction. Emotion. doi:10.1037/emo000033

Gupta, R., Hur, Y. J., \& Lavie, N. (2016). Distracted by pleasure: Effects of positive versus negative valence on emotional capture under load. Emotion, 16(3), 328-337. doi:10.1037/emo0000112

Lakens, D. (2013). Calculating and reporting effect sizes to facilitate cumulative science: a practical primer for t-tests and ANOVAs. Frontiers in Psychology, 4:863. doi:10.3389/fpsyg.2013.00863

Lakens, D. (2017). Equivalence tests: A practical primer for t tests, correlations, and meta-analyses. Social Psychological and Personality Science, 1948550617697177. doi:10.1177/1948550617697177

Lang, P. J., Bradley, M. M., \& Cuthbert, B. N. (2008). International affective picture system (IAPS): Affective ratings of pictures and instruction manual. Technical Report A-8.

Most, S. B., Smith, S. D., Cooter, A. B., Levy, B. N., \& Zald, D. H. (2007). The naked truth: Positive, arousing distractors impair rapid target perception. Cognition and Emotion,_21(5), 964-981. doi:10.1080/02699930600959340

Okon-Singer, H., Tzelgov, J., \& Henik, A. (2007). Distinguishing between automaticity and attention in the processing of emotionally significant stimuli. Emotion, 7(1), 147-157. doi:10.1037/1528-3542.7.1.147

Padmala, S., \& Pessoa, L. (2011). Reward reduces conflict by enhancing attentional control and biasing visual cortical processing. Journal of Cognitive Neuroscience, 23(11), 3419-3432. doi:10.1162/jocn_a_00011

Padmala, S., \& Pessoa, L. (2014). Motivation versus aversive processing during perception. Emotion, 14(3), 450-454. doi:10.1037/a0036112

Padmala, S., Sirbu, M., \& Pessoa, L. (2017). Potential reward reduces the adverse impact of negative distractor stimuli. Social Cognitive and Affective Neuroscience, 12(9), 1402-1413. doi:10.1093/scan/nsx067 


\section{Motivation and Emotional Distraction}

Pessoa, L. (2009). How do emotion and motivation direct executive control? Trends in Cognitive Sciences, 13(4), 160-166. doi:10.1016/j.tics.2009.01.006

Pourtois, G., Schettino, A., \& Vuilleumier, P. (2013). Brain mechanisms for emotional influences on perception and attention: what is magic and what is not. Biological Psychology, 92(3), 492-512. doi:10.1016/j.biopsycho.2012.02.007

Psychology Software Tools, Inc. [E-Prime 2.0]. (2012). Retrieved from http://www.pstnet.com.

Rolls, E. T. (2005). Emotion explained. Oxford: Oxford University Press.

Wei, P., Wang, D., \& Ji, L. (2016). Reward expectation regulates brain responses to task-relevant and taskirrelevant emotional words: ERP evidence. Social cognitive and affective neuroscience, 11(2), 191-203. doi:10.1093/scan/nsv097

Willenbockel, V., Sadr, J., Fiset, D., Horne, G. O., Gosselin, F., \& Tanaka, J. W. (2010). Controlling low-level image properties: the SHINE toolbox. Behavior Research Methods, 42(3), 671-684.

doi:10.3758/BRM.42.3.671 
Motivation and Emotional Distraction

\section{Footnotes}

${ }^{1}$ IAPS images: Neutral: 2026, 2102, 2221, 2305, 2393, 2397, 2411, 2512, 2593, 2595, 2745.1, 2840. Negative: 3015, 3030, 3059, 3103, 3131, 3140, 3150, 3195, 3550.1, 9253, 9405, 9420. Positive: 4658, 4659, 4660, 4668, 4680, 4690, 4693, 4694, 4695, 4697, 4698, 4800.

${ }^{2}$ With the outlier participant included, this reward $\mathrm{x}$ distractor-type $\mathrm{x}$ distractor-valence interaction becomes non-significant, $F(2,142)=2.31, p=.103, \eta_{p}{ }^{2}=.03$; the outlier disproportionately influences the variance of negative distraction in the reward group (included $M=65 \mathrm{~ms}, S D=90 \mathrm{~ms}$; excluded $M=54 \mathrm{~ms}, S D$ $=60 \mathrm{~ms}$ ) reducing the effect of reward on negative distraction, $t(71)=1.63, p=.107, d=.38$, CI $[-7,70]$. Outlier inclusion did not influence the significance of any other findings. 


\section{Motivation and Emotional Distraction}

Table 1. Comparisons of mean correct RTs (ms) and accuracy (proportion correct) for scrambled versus intact images (distraction), for each distractor-valence condition, separately for control and reward groups.

\begin{tabular}{llllll}
\hline Valence & Scrambled & Intact & Distraction & $d_{r m}$ & \multicolumn{2}{c}{ Low CI } \\
Block & Trials & Trials & & &
\end{tabular}

Control Group

$\underline{\text { Response Times }}$

$\begin{array}{lllllll}\text { Negative } & 614(116) & 710(155) & 96^{* * *(72)} & .61 & 72 & 121 \\ \text { Neutral } & 618(120) & 648(131) & 30^{* * *(25)} & .21 & 21 & 38 \\ \text { Positive } & 624(117) & 712(156) & 88^{* * *(70)} & .54 & 64 & 111\end{array}$

\section{$\underline{\text { Reward Group }}$}

$\begin{array}{lllllll}\text { Negative } & 550(80) & 603(119) & 53 * *(60) & .42 & 33 & 74 \\ \text { Neutral } & 551(79) & 573(85) & 22 * * *(18) & .26 & 16 & 28 \\ \text { Positive } & 554(83) & 596(113) & 42 * * *(48) & .34 & 26 & 58\end{array}$

\section{Control Group}

\section{Proportion Correct}

$\begin{array}{lllllll}\text { Negative } & .950(.041) & .902(.080) & .048 * * *(.069) & .70 & .025 & .071 \\ \text { Neutral } & .958(.031) & .942(.048) & .016(.039) & .35 & .002 & .028 \\ \text { Positive } & .949(.045) & .905(.072) & .044 * *(.075) & .72 & .019 & .070\end{array}$

\section{Reward Group}

\begin{tabular}{llllllll} 
Negative & $.899(.067)$ & $.877(.089)$ & .022 & $(.072)$ & .27 & -.002 & .047 \\
Neutral & $.919(.059)$ & $.898(.069)$ & $.021 * *(.044)$ & .32 & .006 & .036 \\
Positive & $.915(.066)$ & $.881(.085)$ & $.034 * *(.070)$ & .44 & .010 & .058 \\
\hline
\end{tabular}

Notes: Distraction is calculated as: [RT intact - RT scrambled] and [proportion-correct scrambled proportion-correct intact]. SDs are in brackets. Effect sizes are Cohen's $d_{r m}$ from paired comparisons of intact and scrambled trials within conditions, and asterisks indicate whether the [intact - scrambled] difference is significant in that condition. ${ }^{*} p<.05,{ }^{*} p<.01, * * * p<.001 .95 \%$ confidence intervals surround the distraction scores, in ms and proportion correct. $n=36$ per group. 
Motivation and Emotional Distraction

Figure 1. (A) Schematic trial sequence. The displays are enlarged to enhance the central portion of the screen and so are not to scale. (B) Mean distraction in ms (RT intact - RT scrambled) is shown for the control and reward groups for each valence condition. Error bars represent $\pm 195 \%$ confidence intervals.

A
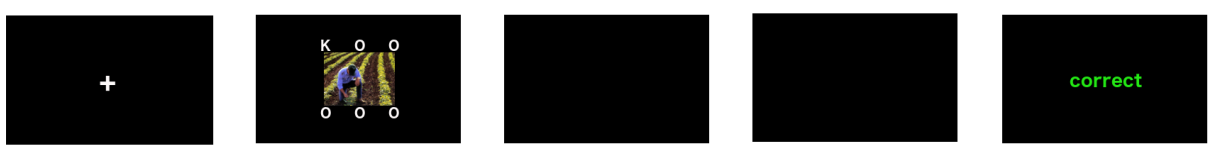

Fixation

(417-833 ms) randomised

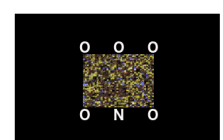

Response window (1800 ms or until response)

Blank screen

(600 ms)

Auditory feedback on

Feedback (100 ms)

successful

Target display (200 ms)

reward trials intact or scrambled

B

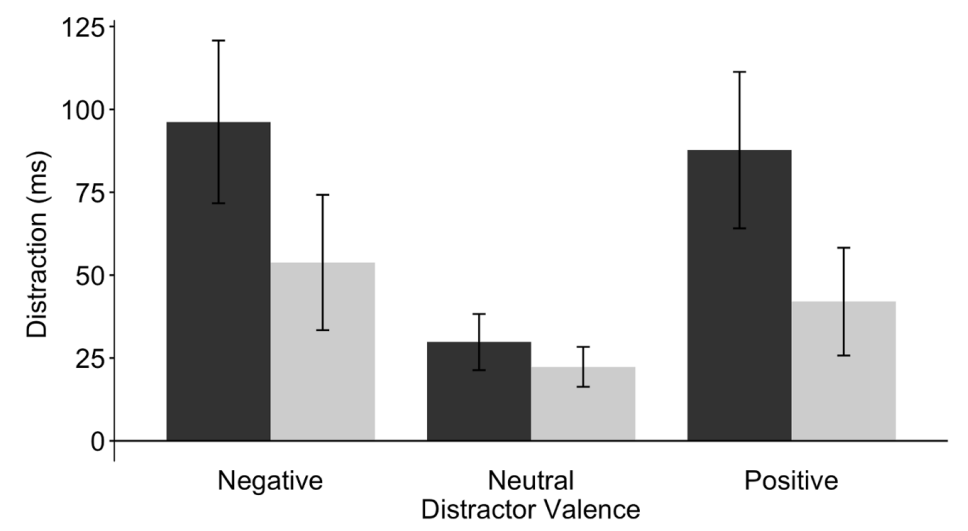

Reward Group

Control

Reward



ITI

(207-623 ms)

randomised 
Motivation and Emotional Distraction

\section{Appendix B.}

Table S.1. IAPS image numbers for each image set for men, and for women. The same images were used across all experiments. However, only Experiment 2 included men and women. Experiments 1, 3, and 4 include only women.

\begin{tabular}{cccccc}
\hline Neutral & Women & & & Men & \\
\hline 2026 & Negative & Positive & Neutral & Negative & Positive \\
2102 & 3015 & 4658 & 2026 & 3000 & 4645 \\
2221 & 3059 & 4659 & 2102 & 3015 & 4650 \\
2305 & 3103 & 4660 & 2104 & 3053 & 4653 \\
2393 & 3131 & 4668 & 2221 & 3060 & 4658 \\
2397 & 3140 & 4690 & 2393 & 3069 & 4660 \\
2411 & 3150 & 4693 & 2411 & 3080 & 4669 \\
2512 & 3195 & 4694 & 2512 & 3100 & 4680 \\
2593 & 3550.1 & 4695 & 2593 & 3120 & 4690 \\
2595 & 9253 & 4697 & 2595 & 3130 & 4692 \\
2745.1 & 9405 & 4698 & 2745.1 & 3131 & 4693 \\
2840 & 9420 & 4800 & 2840 & 9410 & 4698 \\
\hline
\end{tabular}


Motivation and Emotional Distraction

\title{
Appendix C. Preprint of publication for Chapter 3.
}

Walsh, A. T., Carmel, D., \& Grimshaw, G. M. (2018, October 25). Reward elicits cognitive control over emotional distraction: Evidence from pupillometry. Cognitive, Affective, \& Behavioural Neuroscience. https://doi.org/10.3758/s13415-018-00669-w

\section{Reward elicits cognitive control over emotional distraction: Evidence from pupillometry.}

\author{
Amy T. Walsh ${ }^{1}$, David Carmel ${ }^{1,2}$ and Gina M. Grimshaw ${ }^{1}$ \\ ${ }^{I}$ School of Psychology, Victoria University of Wellington, New Zealand \\ ${ }^{2}$ Department of Psychology, University of Edinburgh, $U K$
}

Address Correspondence to: Gina Grimshaw, School of Psychology, Victoria University of Wellington, PO Box600, Wellington 6140, New Zealand. gina.grimshaw@vuw.ac.nz.

Other authors: Amy Walsh, School of Psychology, Victoria University of Wellington, PO Box 600, Wellington 6140, New Zealand, amy.walsh@vuw.ac.nz, +64 04463 6420; David Carmel, School of Psychology, Victoria University of Wellington, PO Box 600, Wellington 6140, New Zealand; and Psychology Department, University of Edinburgh, 7 George Square, Edinburgh EH8 9JZ, UK; dave.carmel@ed.ac.uk, +44-131-650-3455.

Research was supported by a grant from the Royal Society of New Zealand Marsden Fund (VUW-1307) to Gina Grimshaw and David Carmel. Findings were reported at the meeting of the Society for Psychophysiological Research, Vienna, October, 2017. Disclosure of interest statement: The authors report no conflict of interest.

The authors would like to acknowledge contributions from Al Abenoja for technical assistance, Dr. Sanjay Manohar for sharing MATLAB code for pupillometry pre-processing analysis, Dr. Michael C. Hout for sharing code for experiment programming, Cathryn Bjarnesen for assistance with experiment planning, and Dr. Christel Devue for assistance with programming the experiment.

Our experiment pre-registration (osf.io/jd96p/), materials, data, analyses, and code are available on Open Science Framework at: osf.io/yhkdr 
Motivation and Emotional Distraction

\begin{abstract}
Attention is biased toward emotional stimuli, even when they are irrelevant to current goals. Motivation, elicited by performance-contingent reward, reduces behavioural emotional distraction. In emotionallyneutral contexts, reward is thought to encourage use of a proactive cognitive control strategy, altering anticipatory attentional settings to more effectively suppress distractors. The current preregistered study investigates whether a similar proactive shift occurs even when distractors are highly arousing emotional images. We monitored pupil area, an online measure of both cognitive and emotional processing, to examine how reward influences the timecourse of control. Participants $(n=110)$ identified a target letter flanking an irrelevant central image. Images were meaningless scrambles on $75 \%$ of trials; on the remaining $25 \%$, they were intact positive (erotic), negative (mutilation), or neutral images. Half the participants received financial rewards for fast and accurate performance, while the other half received no performance-contingent reward. Emotional distraction was greater than neutral distraction, and both were attenuated by reward. Consistent with behavioural findings, pupil dilation was greater following emotional than neutral distractors, and dilation to intact distractors (regardless of valence) was decreased by reward. Although reward did not enhance tonic pupil dilation (an index of sustained proactive control), exploratory analyses showed that reward altered the timecourse of control - eliciting a sharp, rapid, increase in dilation immediately preceding stimulus-onset (reflecting dynamic use of anticipatory control), that extended until well after stimulus-offset. These findings suggest that reward alters the timecourse of control by encouraging proactive preparation to rapidly disengage from emotional distractors.
\end{abstract}

Keywords: emotion, motivation, distraction, cognitive control, reward, pupillometry 


\section{Motivation and Emotional Distraction}

Emotional distractions can be difficult to ignore (Pourtois, Schettino, \& Vuilleumier, 2013;

Okon-Singer, Tzelgov, \& Henik, 2007; Yiend, 2010). Although attentional biases to emotional stimuli are often thought of as reflexive (e.g., Brosch, Pourtois, Sander, \& Vuilleumier, 2011) increasing task motivation via performance-contingent reward can enhance cognitive control of emotional distractions (Padmala \& Pessoa, 2014; Padmala, Sirbu, \& Pessoa, 2017; Walsh, Carmel, Harper, \& Grimshaw, 2018), just as motivation enhances control in emotionally-neutral contexts (Chiew \& Braver, 2013, 2014; Locke \& Braver, 2008; Padmala \& Pessoa, 2011). However, we know little about the cognitive mechanisms by which motivation enhances control of emotional distractions. The current study uses pupillometry, a temporally-sensitive measure of both cognitive control and emotional processing (Beatty \& Lucero-Wagoner, 2000; Bradley, Miccoli, Escrig, \& Lang, 2008; Laeng, Sirois, \& Gredeback, 2012), to address the question of how reward influences the timecourse of control when distractors are emotional.

The Dual Mechanisms of Control Framework (DMC; Braver, Gray, \& Burgess, 2007; Braver, 2012), which is usually applied in emotionally-neutral contexts, provides predictions as to how motivation might influence the control of emotional distractions. Cognitive control allows us to focus on task-relevant information while ignoring task-irrelevant distractions. Often, we control distraction using a reactive control strategy, disengaging from distractors after they capture our attention (Geng, 2014). Proactive control requires greater effort, because it involves anticipatorily preparing to suppress distractors before they appear. Neurally, proactive control recruits lateral PFC to actively maintain representations of goals prior to stimulus-onset (Braver, 2012; Braver, Paxton, Locke, \& Barch, 2009). This activation can be sustained over time, or dynamically implemented before stimulus-onset. Reactive control also recruits lateral PFC, but does so after a distractor has appeared (Braver, 2012; Braver et al., 2009). The terms "reactive" and "proactive" here refer to the timing of the implementation of control, and not to specific mechanisms per se. Proactive mechanisms could include, for example, anticipatory enhancement of target representations, or suppression of distractor location or features; reactive mechanisms could include facilitation of conflict detection, or a speeding of disengagement.

A central tenet of the DMC framework is that we shift to more effective, but more effortful, proactive control only when it is worthwhile to do so (see Botvinick \& Braver, 2015 for a review). Reward is one incentive that is known to motivate a shift to proactive control in emotionally-neutral contexts (Chiew \& Braver, 2013, 2014; Fröber \& Dreisbach, 2014; 2016; 
Motivation and Emotional Distraction

Hefer \& Dreisbach, 2016; Locke \& Braver, 2008; Padmala \& Pessoa, 2011; Yamaguchi \& Nishimura, 2018). This proactive shift is thought to be implemented via connections between reward-processing areas (such as the ventral striatum and orbitofrontal cortex) and cognitive control regions (such as the anterior cingulate cortex and dorsolateral prefrontal cortex; Botvinick \& Braver, 2015; Pessoa \& Engelmann, 2010; Pessoa, 2009). However, it is not known whether motivation similarly enhances control of emotional distraction via a shift to proactive control.

It is reasonable to think that reward might enhance control of emotional distractions via different mechanisms than it does for neutral distractions. Attention is biased toward biologically-relevant emotional stimuli because they are important for survival and reproduction (LeDoux, 2012). Therefore, it might be adaptive for them to always capture our attention, regardless of their relevance to current goals, so that we can determine whether they require immediate action before we disengage from them. Emotional distractors (unlike their neutral counterparts) are thought to bias our attention via both exogenous (reflexive) and endogenous (goal-driven) processes (Brosch et al., 2011; Mohanty \& Sussman, 2013; Mulckhuyse, 2018; Sussman, Jin, \& Mohanty, 2016), making them more difficult to ignore (Pourtois et al., 2013; Okon-Singer et al., 2007; Yiend, 2010). If emotional distractors bias our attention reflexively, we may not be able to control them proactively. Instead, we may be more likely to control emotional distraction by up-regulating reactive control so that we can rapidly disengage from distractors. Our primary research question is therefore whether the availability of rewards encourages proactive control, just as it does in emotionally-neutral contexts.

Recent neuroimaging studies provide some support for the proactive control of emotional distraction under reward. In a series of studies Padmala and colleagues (Padmala \& Pessoa, 2014; Padmala et al., 2017) had participants ignore a centrally-presented distractor image while judging the orientation of two flanking lines. Participants were slower when negative compared to neutral distractors were presented (i.e., they showed an emotional distraction effect). On some trials, a pre-trial reward cue appeared, indicating availability of monetary reward for fast and accurate performance on the upcoming trial. Reward sped up responses, and also eliminated emotional distraction. Functional magnetic resonance imaging (fMRI) showed that participants with greater ventral striatum activation during the reward cue phase showed a greater benefit from reward on a behavioural measure of emotional distraction (Padmala et al., 2017). Furthermore, there was stronger functional connectivity between ventral striatum and frontal and parietal regions involved in attentional control while 
Motivation and Emotional Distraction

processing reward compared to non-reward cues, consistent with the recruitment of proactive control mechanism to reduce emotional distraction when rewards were signalled.

Although neuroimaging findings support the hypothesis that reward encourages the implementation of proactive control, dynamic changes in control are best examined using more temporally-sensitive measures of cognitive and emotional processing. The current preregistered study uses pupillometry (a high-resolution measure of changes in pupil area over time), to examine the effect of reward on the timecourse of cognitive control of emotional and neutral distractions. Pupil dilation marks changes not only in perceived luminance (Mathôt \& Van der Stigchel, 2015), but also in cognitive processing; dilation is an established, temporally-sensitive, measure of cognitive control (Beatty \& Lucero-Wagoner, 2000; Chatham, Frank, \& Munakata, 2009; Chevalier, Martis, Curran, \& Munakata, 2015; Chiew \& Braver, 2013, 2014; Kahneman, 1973; Jones, Siegle, \& Mandell, 2015; Rondeel, van Steenbergen, Holland, \& van Knippenberg, 2015). Pupil dilation is driven by release of the neuromodulator norepinephrine (NE) from the locus coeruleus (LC; Joshi, Kalwani, \& Gold, 2016; Laeng et al., 2012; Rajkowski, Majczynski, Clayton, \& Aston-Jones, 2004). The LC (located in the rostral pons) provides the major source of NE to the brain, sending dense projections to diverse brain regions, including those involved in attentional control (e.g., PFC, parietal cortex, superior colliculus, and the pulvinar nucleus) and reward processing (including VTA, eliciting dopaminergic release from the nucleus accumbens; see Laeng et al., 2012; Sara, 2009 for reviews). It is thought that NE has an important arousal-inducing function, which promotes cognitive performance (Laeng et al., 2012; Samuels \& Szabadi, 2008; Sara, 2009). Furthermore, NE is thought to facilitate changes to attention, cognition and behaviour by enhancing functional connectivity between various brain regions (Coull, Buchel, Friston, \& Frith, 1999; Sara, 2009). Pupillometry is sensitive to both emotional arousal (e.g., Bradley et al., 2008) and cognitive effort (see Kahneman, 1973; Laeng et al., 2012), and so is well-suited to our aims.

Two measures of pupil dilation are relevant: tonic dilation refers to changes in baseline pupil area (often measured during an inter-trial-interval period; e.g., Chiew \& Braver, 2013, 2014; Heitz, Schrock, Payne, \& Engle, 2008), and reflects state-related changes such as increases in sustained arousal; phasic dilation is an event-related transient change triggered by a specific stimulus or event (baselined to a preceding time-period). Recently, Chiew and Braver $(2013,2014)$ used both tonic and phasic pupil dilation to measure the 


\section{Motivation and Emotional Distraction}

timecourse of motivationally-induced enhancements of cognitive control. Participants were rewarded for successful performance on a cognitive control task (AX Continuous Performance), which elicited behavioural performance consistent with a shift toward proactive control. Reward increased tonic pupil dilation, thought to reflect increases in arousal that accompany a shift to a sustained proactive control strategy. Reward also increased preparatory phasic pupil dilation (triggered by the cue for an impending target), thought to reflect a more dynamic form of proactive control (Chatham et al., 2009; Chevalier et al., 2015). These findings suggest that both tonic and phasic changes in pupil dilation can mark a motivationally-driven shift to proactive control, at least in an emotionally-neutral context.

Pupil dilation also indexes emotional arousal induced by viewing emotional images (Bradley et al., 2008; Cohen, Moyal \& Henik, 2015; Henderson, Bradley, \& Lang, 2014; Kinner et al., 2017; Snowden et al., 2016; Vanderhasselt, Remue, Ng, \& De Raedt, 2014), and correlates with measures of skin conductance (Bradley et al., 2008). This emotional pupil response is subject to top-down influences. When cognitive control is enhanced via conflict manipulations (flanker incongruence; Cohen, et al., 2015); or by implementation of effective emotion-regulation strategies (Kinner et al., 2017; Vanderhasselt, et al., 2014), pupil dilation in response to negative emotional images is reduced. Furthermore, pupil dilation during anticipation of a negative emotional image is inversely related to pupil dilation in response to the image itself (Vanderhasselt et al., 2014). Therefore, increased anticipatory pupil dilation can index proactive control of emotional processing; and reduced post-stimulus pupil dilation can index attenuated processing of emotional content.

The current study takes advantage of the pupil's sensitivity to the timing of cognitive effort and emotional processing, to determine whether reward reduces emotional distraction via a shift to proactive control. Participants identified a target letter flanking an irrelevant central image. The behavioural task is a replication of that used by Walsh et al. (2018) to show that reward attenuates distraction by both positive and negative distractors. Images were meaningless scrambles on $75 \%$ of trials, a proportion that gives rise to a predominantly reactive control strategy (Bugg \& Crump, 2012; Grimshaw, Kranz, Carmel, Moody, \& Devue, 2018); on the remaining 25\% of trials, however, intact positive (erotic couples), negative (mutilations), or neutral images (of people) were presented. Distractor valence was blocked, so that participants could anticipate the type of distractor they might see, but not on which trial an intact distractor would occur. Half the participants received performance- 
Motivation and Emotional Distraction

contingent financial rewards for fast and accurate performance, while the other half received a fixed payment that was not linked to performance.

We expected that the motivational manipulation would enhance control, reflected by reduced behavioural emotional distraction (replicating Walsh et al., 2018), and a reduction in the pupil's response to emotional distractors. Our key question was whether reward improves performance by encouraging people to shift to a proactive control strategy to ignore emotional distractors. If reward elicits proactive control via a global strategy that is maintained across an experimental block, we expect reward to increase tonic pupil dilation. If reward elicits proactive control via a dynamic strategy that occurs on a trial-by-trial basis, we expect reward to enhance phasic, pre-stimulus pupil dilation. Additionally, we examined phasic pupil dilation in the post-stimulus period, to provide insight on the consequences of control for subsequent processing of distractors. Specifically, we predicted that reward would reduce the dilation to intact (relative to scrambled) distractors, reflecting reduced distractor processing. Lastly, we examined reward x valence interactions in all planned analyses, to determine whether reward influences the timecourse of control (indexed by pupil dilation) of neutral, negative and positive distractions similarly.

\section{Method}

This study was preregistered on Open Science Framework (OSF) prior to data collection (osf.io/jd96p/). The preregistration adheres to the disclosure requirements of OSF. All data, materials, and code used for image manipulation and statistical analyses, are available at osf.io/yhkdr.

\section{Sample size determination}

To determine the sample size needed to test the effect of reward on the pupil's response to emotional images, we assumed that the effect of reward on post-stimulus pupil dilation would be comparable in size to the effect of reward on a behavioural measure of emotional distraction (RT), which was $d=.519$ in Walsh et al. (2018). This effect size is similar to that reported by Cohen et al. $(2015 ; d=.503)$ for the effect of flanker congruency on pupillary response to negative (relative to neutral) images. A sample size analysis in G*Power (Faul, Erdfelder, Lang, and Buchner, 2007) indicated 47 participants per group would provide sufficient power to detect a similar effect of reward on emotional distraction $80 \%$ of the time (alpha $=.05)$. Rounding up to accommodate counterbalancing yielded a sample size of 54 participants per reward condition. A sensitivity analysis (also in G*Power, 
Motivation and Emotional Distraction

with alpha $=.05)$ showed that 54 participants per group provide sensitivity to detect an effect of $d=.482$ or larger at $80 \%$ power.

\section{Participants}

A total of 114 participants were recruited from the community in Wellington, New Zealand, via fliers and emails. Participants reported normal vision, spoke fluent English, and were not currently being treated for depression or anxiety. Participants were randomly allocated to the control or reward groups. Four participants did not complete the task due to technical errors (experiment crashing $n=3$, eye-tracker technical issues $n=1$ ). Replacement participants were run to reach the sample size goal, resulting in 55 participants in each group (control group: 13 men, 42 women; reward group: 18 men, 37 women; between 18-39 years old, $M=23, S D=4)$. We exceeded the target of 54 participants per condition because a participant who was mistakenly excluded and rerun did not actually meet the preregistered exclusion criteria; an additional participant was therefore recruited to balance the size of the control and reward groups. The study was approved by the School of Psychology Human Ethics Committee, by delegated authority of the Victoria University of Wellington Human Ethics Committee. Participants provided informed consent prior to participation.

\section{Stimuli and Materials}

The experiment was run on an Acer PC with a 22" flat-screen monitor with $1024 \mathrm{x}$ 768 pixel resolution and a $120 \mathrm{~Hz}$ refresh rate. Viewing distance was maintained by a chinrest $60 \mathrm{~cm}$ from the screen. The experiment was programmed and run on E Prime 2.0 (Psychology Software Tools, Pittsburgh, PA). A nine-point calibration and validation of the eye-tracker was performed at the beginning of the session. Between each block a manual drift check was performed, and if necessary the calibration and validation were performed again. Area of the left pupil (in arbitrary units) was recorded using an Eyelink 1000-plus desktop mounted eye-tracker (SR Research Ltd., Mississauga, ON) using a $1000 \mathrm{~Hz}$ sampling rate.

The images were selected from the International Affective Picture System (IAPS; Lang, Bradley, \& Cuthbert, 2008) and comprised 12 high arousal negative images (gory images of mutilations), 12 high arousal positive images (erotic images of heterosexual couples), and 12 neutral images (of people). So that the positive and negative image sets could be matched on arousal, separate but overlapping image sets were selected for men and women. See supplementary materials for the valence and arousal ratings for the image sets (Table S.1) and specific IAPS images used and the mean ratings for the image sets (Table S.2). 
Motivation and Emotional Distraction

Three scrambled versions of each image were used as control stimuli (see an example in Figure 1). The scrambled images were created in MATLAB by performing a 2D fast Fourier transform, followed by phase randomisation, and reconstruction of the image. The process of phase scrambling also matched the images on average RMS contrast. The scrambled images maintain the same lower level visual properties (i.e. colour, amplitude spectrum) as the original image while changing the position of the spatial frequency components and removing meaningful content. All images (intact and scrambled) were matched on average luminance using the SHINE MATLAB toolbox (Willenbockel et al., 2010).

The target display (see Figure 1A) consisted of a centrally presented image $\left(11^{\circ}\right.$ width $x 8.26^{\circ}$ height $)$ on a grey background, with six white letters $\left(0.86^{\circ} \times 0.92^{\circ}\right)$ placed $0.75^{\circ}$ above and three $0.75^{\circ}$ below the image's horizontal edge. In the centre of the image was a light grey cross which provided a focal point for participants to help them control eyemovements. The image was an intact IAPS image on $25 \%$ of trials, and a scrambled image on $75 \%$ of trials. On each trial, five of the letters were Os and the target was a K or N. The target letter appeared in each location an equal number of times. The display was similar to that used in Gupta, Hur, \& Lavie, (2016), and Walsh et al. (2018).

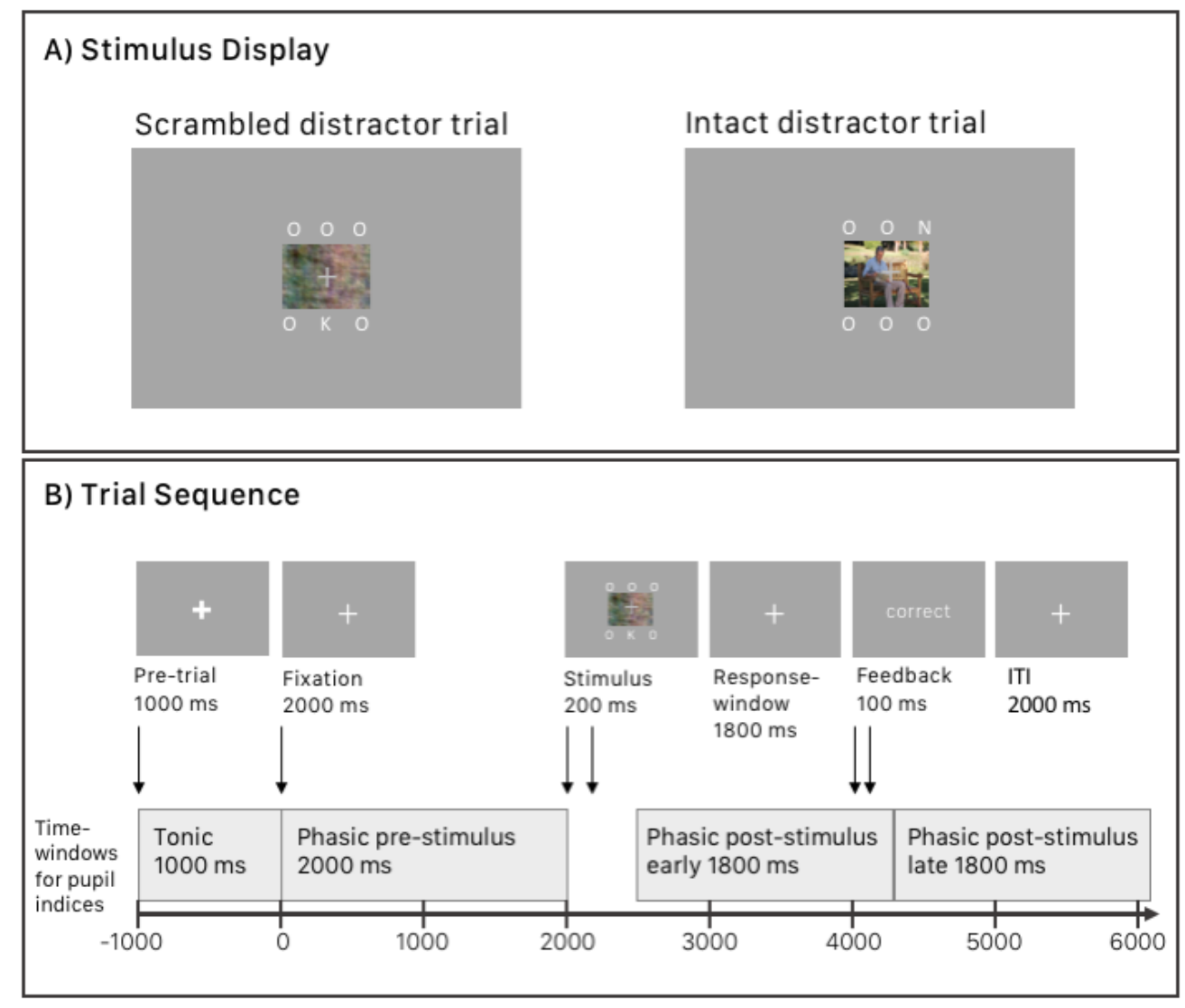


Motivation and Emotional Distraction

Figure 1. Target display (A) and trial sequence (B). A) An example of a scrambled (left) and intact (right) target display. B) The trial sequence. Arrows indicate onset of a display. Preregistered time-windows for pupil analyses are represented by grey shaded boxes. For illustrative purposes, images are not to scale; the central part of the display is enlarged.

\section{Task and procedure}

Participants were tested in a dimly-lit room. Participants were told that the images were irrelevant to their task, so they should ignore them. They were asked to avoid blinking excessively, to keep their head still, and to continue looking at the centre of the screen throughout a trial.

For all participants (in both reward and control conditions), each trial (see Figure 1B) began with a large, bold white fixation cross on a grey background. When they were ready to start the trial, the participant looked directly at the bold cross for $1000 \mathrm{~ms}$, at which point it turned into a smaller white fixation cross indicating the start of the trial. The smaller fixation cross was presented for a constant duration of $2000 \mathrm{~ms}$ and was followed by the target display for $200 \mathrm{~ms}$. Participants indicated (as quickly and accurately as possible) whether the target letter was a $\mathrm{K}$ or an $\mathrm{N}$, using the keys 1 and 2 on the number pad, with the index and middle fingers of their right hand. Following the target display, the fixation cross reappeared for $1800 \mathrm{~ms}$ (a fixed response-window, regardless of when a response was made). Feedback was then displayed for $100 \mathrm{~ms}$, indicating whether the participant was "correct" or "incorrect" in white font. If no response was made within the response-window then the feedback stated: "please respond faster". Following the feedback, the smaller fixation cross appeared again throughout the $2000 \mathrm{~ms}$ inter-trial-interval (ITI). At the end of a trial, the larger bold fixation cross again appeared, and the next trial began when the participant had fixated on it for $1000 \mathrm{~ms}$.

The experiment began with a set of 12 practice trials, arranged in three mini-blocks (negative, neutral, positive) of four trials each, with one intact and three scrambled images appearing randomly in each mini-block. The images presented in the practice trials were different from those used in experimental blocks. Following the practice trials was a baseline block of 24 trials. The purpose of the baseline block was to calculate participants' individual median RT, to be used as a threshold for reward during the experimental blocks and to provide a baseline from which to measure tonic pupil dilation in subsequent blocks. The baseline block consisted of 24 scrambled distractor trials. The phase-scrambled images used in the baseline block were derived from a set of 12 neutral images (different from those used in the neutral experimental block) and were presented once each. Following the baseline 


\section{Motivation and Emotional Distraction}

block, participants completed six experimental blocks of 48 trials each. Each block comprised 12 different intact and 36 scrambled image trials (in random order), all with one valence. There were two sequential blocks of each valence (e.g. positive, positive, negative, negative, neutral, neutral), resulting in each intact and scrambled image being presented twice in total. Valence order was counterbalanced across participants.

The Behavioural Activation and Inhibition Scale (Carver \& White, 1994) was administered to participants at the end of the experimental session, however due to a technical error the responses were not recorded and were therefore not analysed.

\section{Reward Manipulation}

Following the baseline block, the reward group was informed of the potential to earn points and move up levels in the following six blocks of trials. They were told that winning points would lead to achieving 'levels', and that each new level increased total earnings upon task completion by $\$ 2.50$. Participants began on level one $(\$ 10)$. Level two required 53 points $(\$ 12.50) ; 53$ additional points were needed to ascend to each new level, up to level $6(\$ 20)$. All participants actually earned $\$ 20$ in supermarket vouchers at the end of the study, regardless of the amount they presumed they had won during the study. Feedback was provided after every trial: if participants responded accurately and faster than their median response time in the baseline block, they earned 1 point, and saw: "you won a point!"; if they were accurate but slower than the reward threshold they saw "correct", and if they were incorrect saw "incorrect". Feedback on overall performance was provided in each break between blocks. If a participant moved up a level in the preceding block, the feedback screen read, "Well done! You have moved up a level." If not, it read "No level up". On the same screen, they saw their current point total, current level, and mean proportion correct for the previous block. Participants were reminded at this point to be fast and accurate. To minimise the potential for a speed-accuracy trade-off due to the reward RT deadline, a warning to keep trying to be accurate was provided during the breaks between blocks if accuracy fell below $95 \%$ across the previous block.

The control group performed the task with no performance-contingent motivational incentive. They received informative feedback after each trial ("correct", "incorrect", or "please respond faster"). They did not have the opportunity to earn points and did not receive overall performance feedback between blocks. Instead they saw a screen saying, "please take a short break".

\section{Preregistered analyses}


Motivation and Emotional Distraction

The following descriptions of data processing, calculation of indices, inference and exclusion criteria, and analyses were all preregistered.

\section{Pupillometry data processing}

Pupillometry data were pre-processed in MATLAB using a combination of open source code (see acknowledgements), and our own code. Blinks $<500 \mathrm{~ms}$ long were linearly interpolated. Partial blinks were identified by extreme (unrealistic) changes in pupil area from one time point to the next; pupil area during these partial blinks was then linearly interpolated. Pupil indices were calculated using mean pupil area (in arbitrary units) across several time-windows (as outlined in more detail below). Pupil indices were calculated using mean pupil area (in arbitrary units) across several time-windows. Baseline subtraction was used to calculate indices, as it is more robust to baseline artifacts, and increases statistical power, relative to divisive baseline corrections (Mathôt, Fabius, Van Heusden, \& Van der Stigchel, 2017). Only trials with correct responses were included in phasic pupil indices.

The first two time-windows (tonic and phasic pre-stimulus; see Figure 1B) provide indices to test predictions regarding the effects of reward on anticipatory, proactive control. The first window provides an index of tonic proactive control: mean pupil area across the $1000 \mathrm{~ms}$ time-window prior to trial-onset, baselined to the grand mean of pupil area across the $200 \mathrm{~ms}$ time-window immediately preceding trial-onset across all 24 (non-reward, scrambled image only) baseline block trials. The second window provides an index of phasic proactive control: mean pupil area across the $2000 \mathrm{~ms}$ time-window starting from fixationonset, and ending at stimulus-onset, baselined to mean pupil area across the $200 \mathrm{~ms}$ timewindow immediately preceding fixation-onset. This phasic proactive control index reflects dynamic, effortful preparation immediately preceding stimulus-onset.

Two phasic post-stimulus (early and late) indices were created to test predictions regarding how reward affects the pupil's response to the emotional distractors. The early post-stimulus window begins $500 \mathrm{~ms}$ post-stimulus-onset and extends for $1800 \mathrm{~ms}^{9}$. The late post-stimulus window begins immediately following the first and extends for $1800 \mathrm{~ms}$ until the end of the trial. For both the early and late post-stimulus indices, the baseline is the mean pupil area $200 \mathrm{~ms}$ immediately preceding stimulus-onset. These post-stimulus phasic indices for intact image trials are subtracted from the phasic indices on scrambled image trials (thus controlling for pupil dilation in response to low-level visual properties) to obtain an index of

\footnotetext{
${ }^{9}$ Other (unpublished) studies from our lab, in which participants passively viewed these same images, typically find that effects of distractor type (intact versus scrambled) and valence did not begin to emerge until $500 \mathrm{~ms}$ post-stimulus-onset.
} 
Motivation and Emotional Distraction

the pupil's response to the content of the intact images. All indices were calculated for each individual participant, separately for each valence.

\section{Data exclusion criteria}

Trials were excluded if they were missing more than $50 \%$ of pupil samples, Additionally, trials were excluded from specific analyses if the baseline period for that timewindow was missing more than $50 \%$ of its pupil area samples. A mean of 13 (out of 288) indices were removed per participant $(\min =0, \max =66$; see OSF materials for more information on the breakdown of trial exclusions). Our pre-registration stated that participants would be excluded if they had less than $75 \%$ accuracy overall, less than $60 \%$ accuracy on a given block, loss of more than $20 \%$ of their pupil samples overall, or had fewer than 10 trials in a given condition. No participants met these exclusion criteria.

\section{Inference criteria}

$P$ values $<.05$ were accepted as statistically significant and relevant follow-up analyses were conducted on these effects. One- or two-tailed tests for planned comparisons were specified in the predictions, and were not corrected for multiple comparisons (see Armstrong, 2014). Exploratory analyses were not corrected for multiple comparisons (except for the pupil - behaviour correlations), but are noted as exploratory and should be interpreted with caution. Note that distractor type (intact, scrambled) is not a factor in the analyses because it is either irrelevant (for the proactive indices), or included in the transformations (for the post-stimulus indices). Cohen's $d$ effect sizes are $d_{z}$ for within-subjects comparisons, and $\mathrm{d}_{s}$ for between-subjects comparisons.

\section{Results}

All analyses were preregistered unless noted as exploratory. All preregistered predictions are confirmed as supported, or not supported. Significant findings that were not predicted are noted as such. Data and code to reproduce analyses and figures are provided on OSF (link: osf.io/yhkdr).

\section{Behavioural distraction}

Mean response times (RTs) and proportion correct scores are included in Table 1. Trials with no response or with anticipatory responses $(\mathrm{RT}<200 \mathrm{~ms})$ were counted as errors. 
Motivation and Emotional Distraction

Table 1. Comparisons of mean correct RTs (ms) and accuracy (proportion correct) for scrambled versus intact images (distraction in milliseconds), for each distractor valence condition, separately for control and reward groups.

\begin{tabular}{lllllll}
\hline Valence & Scrambled & Intact & Distraction & $d_{z}$ & \multicolumn{2}{c}{$95 \%$ CI } \\
Block & Trials & Trials & & & Low & Upper \\
\hline
\end{tabular}

\section{$\underline{\text { Response Times }}$}

\section{Control Group}

\begin{tabular}{lllllll} 
Negative & $669(91)$ & $813(152)$ & $144 * *(93)$ & 1.550 & 119 & 169 \\
Neutral & $670(81)$ & $726(112)$ & $56^{* * *(49)}$ & 1.145 & 43 & 69 \\
Positive & $674(94)$ & $825(160)$ & $151^{* * *(93)}$ & 1.636 & 126 & 176 \\
\multicolumn{2}{l}{ Reward Group } & & & & & \\
Negative & $562(100)$ & $620(140)$ & $58^{* * *(60)}$ & 0.978 & 42 & 75 \\
Neutral & $564(86)$ & $598(117)$ & $34 * *(42)$ & 0.824 & 23 & 46 \\
Positive & $568(111)$ & $631(145)$ & $63 * * *(52)$ & 1.204 & 49 & 77
\end{tabular}

\section{$\underline{\text { Proportion Correct }}$}

\section{Control Group}

\begin{tabular}{lccllll} 
Negative & $.965(.036)$ & $.947(.052)$ & $.018^{*}(.055)$ & 0.327 & .003 & .033 \\
Neutral & $.973(.030)$ & $.966(.046)$ & $.007(.044)$ & 0.156 & -.005 & .019 \\
Positive & $.969(.025)$ & $.926(.070)$ & $.043 * * *(.070)$ & 0.631 & .025 & .063 \\
Reward Group & & & & & \\
Negative & $.955(.037)$ & $.915(.081)$ & $.040^{* * *}(.072)$ & 0.560 & .021 & .059 \\
Neutral & $.960(.042)$ & $.946(.054)$ & $.014 *(.045)$ & 0.314 & .002 & .026 \\
Positive & $.952(.038)$ & $.909(.083)$ & $.043 * * *(.075)$ & 0.567 & .063 & .022 \\
\hline
\end{tabular}

Note: Distraction is calculated as: [RT intact-RT scrambled] and [proportion-correct scrambledproportion-correct intact]. SDs are in brackets. Effect sizes are Cohen's $d_{z}$ from paired comparisons of intact and scrambled trials within conditions, and asterisks indicate whether the [intact scrambled] difference is significant in that condition. ${ }^{*} p<.05, * * p<.01, * * * p<.001 .95 \%$ confidence intervals surround the distraction scores, in ms and proportion correct. $n=55$ per group. 
Motivation and Emotional Distraction

Examination of means suggests that (as predicted) reward elicits a speed-accuracy trade-off, speeding up responses at a small cost to accuracy. This speed-accuracy trade-off is examined in supplementary analyses (in which preregistered ANOVAs are performed on correct RTs and proportion correct, and an exploratory ANOVA is conducted on inverseefficiency scores; Bruyer \& Brysbaert, 2011), in which we show that it does not account for the effect of reward group on emotional distraction. Therefore, we followed our preregistered plan and focused our analyses on distraction indices, which were calculated as: ((mean RT intact - mean RT scrambled) / mean RT scrambled)) x 100 (Figure 2). These distraction indices reflect percentage slowing due to intact distractors, relative to performance on scrambled trials. A simple difference score between intact and scrambled RTs was not used because of the overall difference in RTs between the reward and control groups. However, for ease of interpretation, we present the difference scores in milliseconds [intact - scrambled] in Table 1.

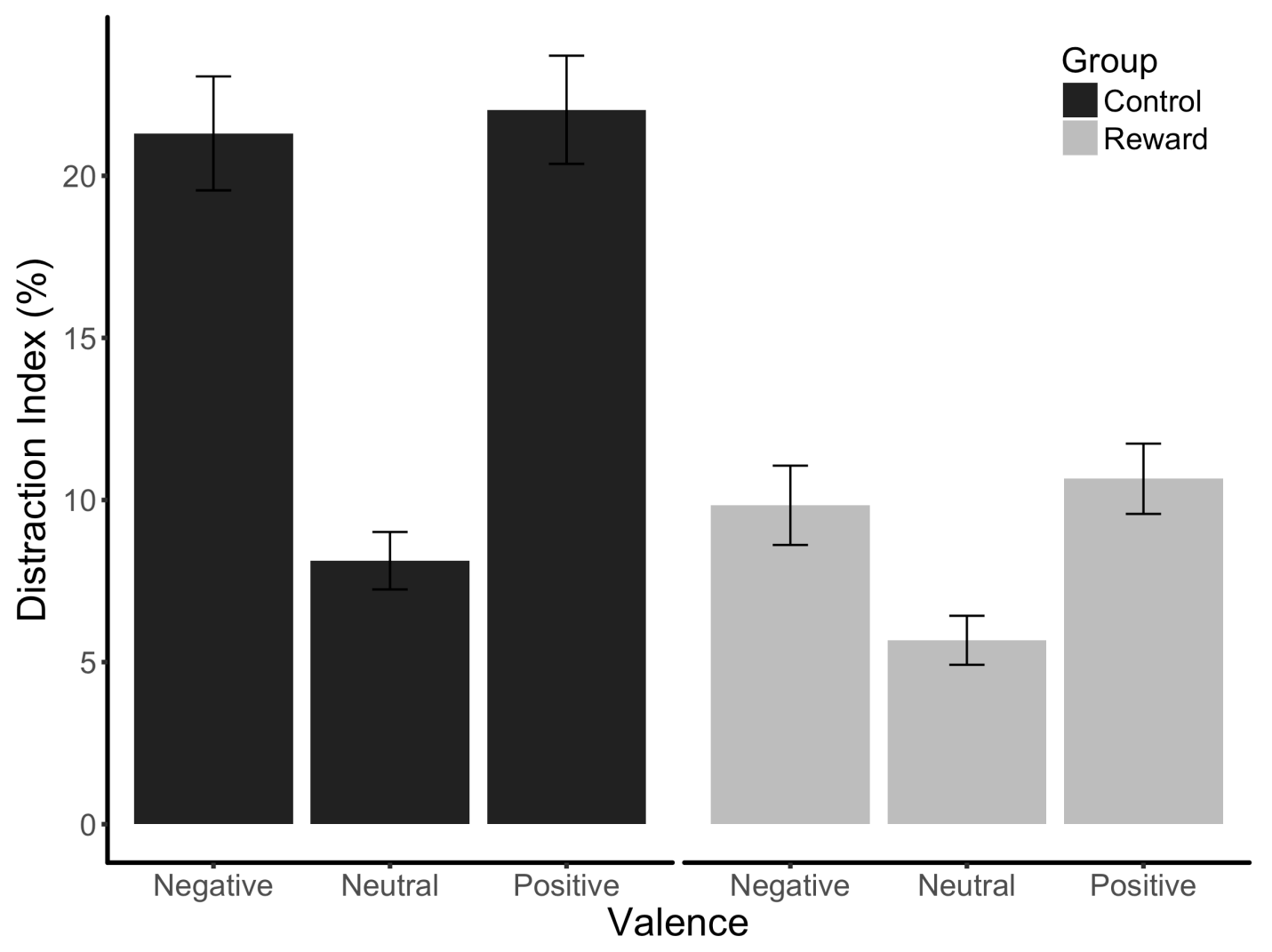

Figure 2. Mean behavioural distraction indices by group and valence. Greater distraction [((mean RT intact - mean RT scrambled) / mean RT scrambled) $x$ 100] was elicited by emotional than by neutral images. Neutral and (to a greater extent) emotional distraction were attenuated by reward. Error bars represent standard error of the mean. 
Motivation and Emotional Distraction

A mixed-model ANOVA was conducted on distraction indices with distractor valence (negative, neutral, positive) as a within-subjects factor and reward group (control, reward) as a between-subjects factor. Neutral valence was entered as the middle level in the ANOVA so that effects of emotional versus neutral distraction could be examined as quadratic effects of valence (as was done in Grimshaw et al., 2018). As predicted, overall, emotional images were more distracting than neutral images, shown by the quadratic main effect of valence: $F(1$, $108)=99.24, p<.001, \eta_{p}{ }^{2}=0.479$. Planned comparisons showed that distraction from positive $(M=16.30 \%, S D=11.82 \%)$ and negative $(M=15.57 \%, S D=12.51 \%)$ images did not differ from each other, $(p=.474$, two-tailed) and so positive and negative distraction were collapsed together into emotional distraction $(M=15.93 \%, S D=10.92 \%)$, which was greater than neutral distraction $(M=6.88 \%, S D 6.19 \%), t(109)=9.04, p<.001, d=0.862,95 \% \mathrm{CI}$ [7.07, inf] (one-tailed).

Crucially, as predicted, the reward group was less distracted overall than the control group: $F(1,108)=38.10, p<.001, \eta_{p}{ }^{2}=0.261$ (controls: $M=17.10 \%, S D=7.85 \%$; reward: $M=8.73 \%, S D=6.28 \%$ ). Also as predicted, reward reduced emotional distraction to a greater degree than it did for neutral distraction, reflected by the quadratic reward $\mathrm{x}$ valence interaction, $F(1,108)=24.366, p<.001, \eta_{p}{ }^{2}=0.184$. There was no difference between positive and negative distraction in either the control or reward groups (both $p$ 's $>.473$, twotailed), so positive and negative distraction were collapsed together for the following planned one-tailed tests. Emotional distraction was significantly reduced in the reward group $(M=$ $10.25 \%, S D=7.82 \%)$ compared to the control group $(M=21.61 \%, S D=12.35 \%), t(108)=$ $6.37, p<.001, d=1.214,95 \%$ CI [8.40, inf] (one-tailed). Neutral distraction was also significantly reduced in the reward group $(M=5.68 \%, S D=5.62 \%)$ compared to the control group $(M=8.08 \%, S D=6.55 \%), t(108)=2.06, p=.021, d=0.392,95 \%$ CI $[0.46$, inf $]$ (onetailed), though to a lesser extent than emotional distraction was.

\section{Pupil data}

Pupil area across the entire trial (baselined against the mean of the $200 \mathrm{~ms}$ pre-trial period) is shown separately for the control and reward groups in Figure 3. 


\section{Whole Trial}

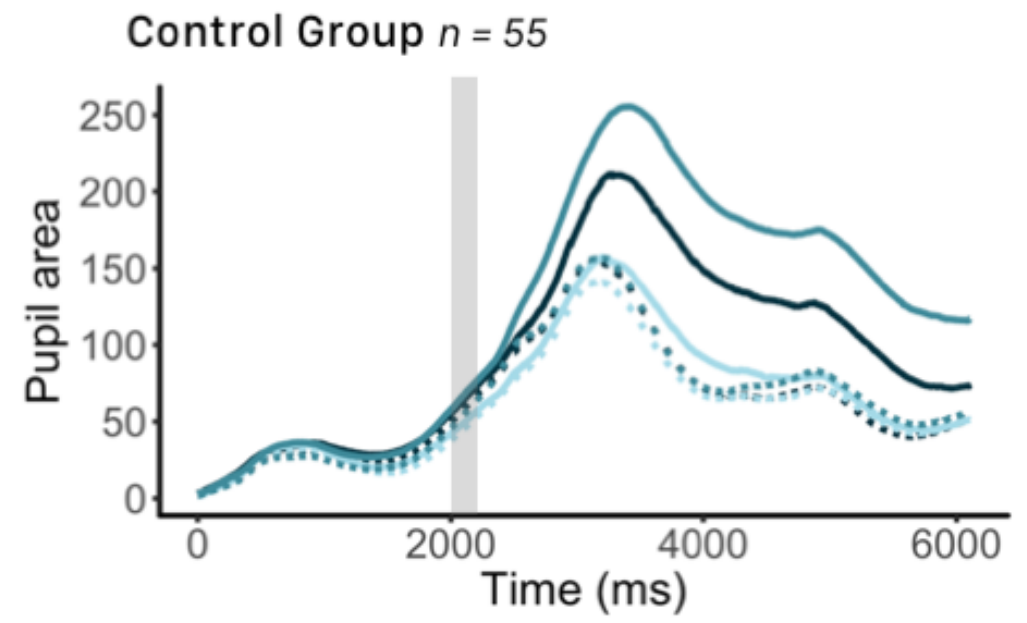

Intact distractor

- Negative

- Neutral

- Positive

\section{Scrambled distractor}

... Negative

Neutral

... Positive

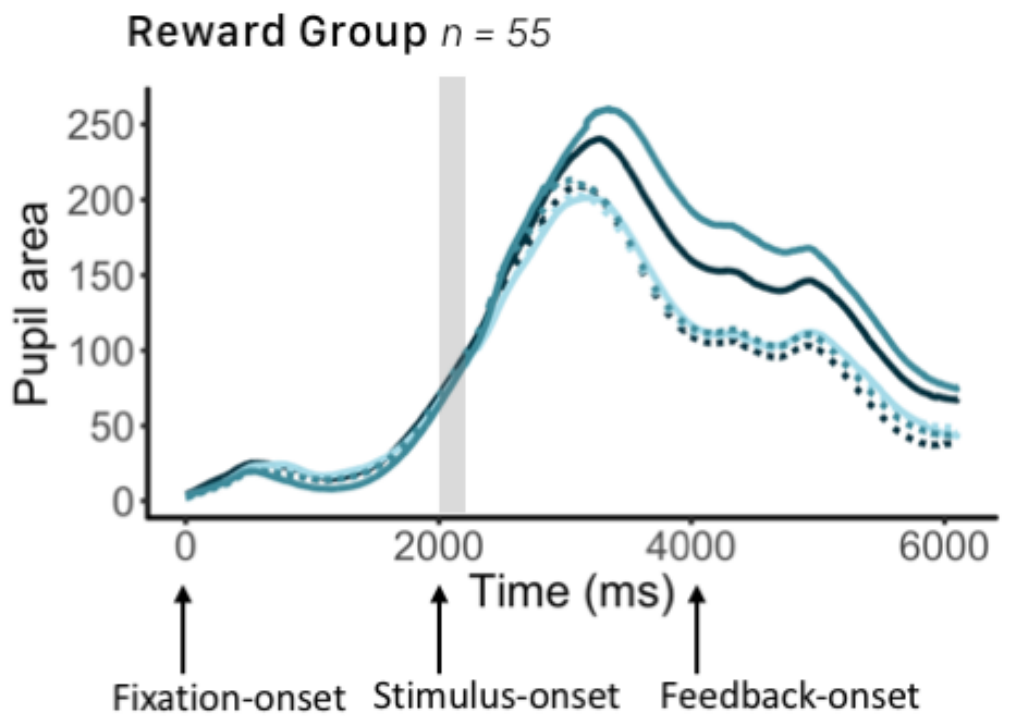

Intact distractor

- Negative Neutral

- Positive

Scrambled distractor

... Negative

Neutral

Positive

Fixation-onset Stimulus-onset Feedback-onset

Figure 3. Mean pupil area (in arbitrary units) across the entire trial. Dilation is subtraction-baselined to the mean during the $200 \mathrm{~ms}$ time-window immediately preceding trial onset (on each trial) for the control group (top panel) and the reward group (bottom panel). Intact trials are represented by solid lines; scrambled images are represented by dotted lines. Distractor valence is represented by the different colour shades. Stimulus presentation $(200 \mathrm{~ms})$ is represented by the grey shaded area. Arrows indicate display-onset for fixation, stimulus, and feedback.

Proactive control pupil indices. See Table 2 for means and $S D$ s for phasic and tonic proactive control indices. Note that the tonic means are all negative values; this is because tonic pupil area decreased across the experimental session in both groups, relative to the preexperimental baseline block at the start of the session. This is not unusual in long experimental sessions. For simplicity, we will refer to differences in dilation between 
Motivation and Emotional Distraction

experimental conditions, however these should be interpreted in light of an overall pattern of constriction in experimental relative to baseline blocks.

Table 2. Mean pupil area in arbitrary units (and $S D$ ) for preregistered tonic and phasic proactive time-windows, for each distractor-valence condition, separately for control and reward groups.

\begin{tabular}{lll}
\hline Valence Block & $\begin{array}{l}\text { Tonic } \\
\text { Proactive } \\
\text { Control }\end{array}$ & $\begin{array}{l}\text { Phasic } \\
\text { Proactive } \\
\text { Control }\end{array}$ \\
\hline Control Group & & \\
Negative & $-108(242)$ & $26(33)$ \\
Neutral & $-121(240)$ & $23(26)$ \\
Positive & $-90(244)$ & $24(30)$ \\
Reward Group & & \\
Negative & $-55(209)$ & $23(28)$ \\
Neutral & $-89(228)$ & $22(26)$ \\
Positive & $-42(165)$ & $20(21)$ \\
\hline
\end{tabular}

Separate mixed-model ANOVAs were conducted on the tonic and phasic proactive control indices, each with valence (negative, neutral, positive) as a within-subjects factor and reward group (reward, controls) as a between-subjects factor. Analysis of the tonic proactive indices (reflecting sustained dilation across blocks, see Figure 4) revealed that, as predicted, there was higher tonic dilation in emotional compared to neutral blocks, as reflected in the quadratic main effect of valence, $F(1,108)=9.03, p=.003, \eta_{p}{ }^{2}=0.077$. Planned comparisons showed that tonic pupil dilation in positive $(M=-66, S D=209)$ and negative $(M$ $=-82, S D=227$ ) blocks did not differ from each other, $t(109)=1.48, p=.141, d=0.141$, 95\% CI [-37, 5] (two-tailed), and so positive and negative tonic dilation were collapsed together into emotional tonic dilation. Tonic dilation was greater on emotional $(M=-74, S D$ $=210)$ than neutral blocks $(M=-105, S D=234), t(109)=3.01, p=.002, d=0.287,95 \% \mathrm{CI}$ $[14$, inf] (one-tailed), reflecting greater sustained arousal in an emotional context. Contrary to predictions, reward did not increase tonic pupil dilation, $F(1,108)=1.21, p=.274, \eta_{p}{ }^{2}=$ 0.011 (although numerically tonic dilation was higher in the reward group compared to the control group), and there was no reward group $\mathrm{x}$ valence interaction, $F(2,216)=0.46, p=$ $.629, \eta_{p}{ }^{2}=0.004$ (quadratic interaction: $F(1,108)=0.814, p=.369, \eta_{p}{ }^{2}=0.007$ ). Thus, tonic dilation was modulated by emotional context, consistent with a shift to proactive control on 
Motivation and Emotional Distraction

emotional blocks (but see Discussion for an alternative explanation). However, tonic dilation was not modulated by reward group, suggesting that reward does not elicit sustained proactive control.

\section{Tonic Time-window}
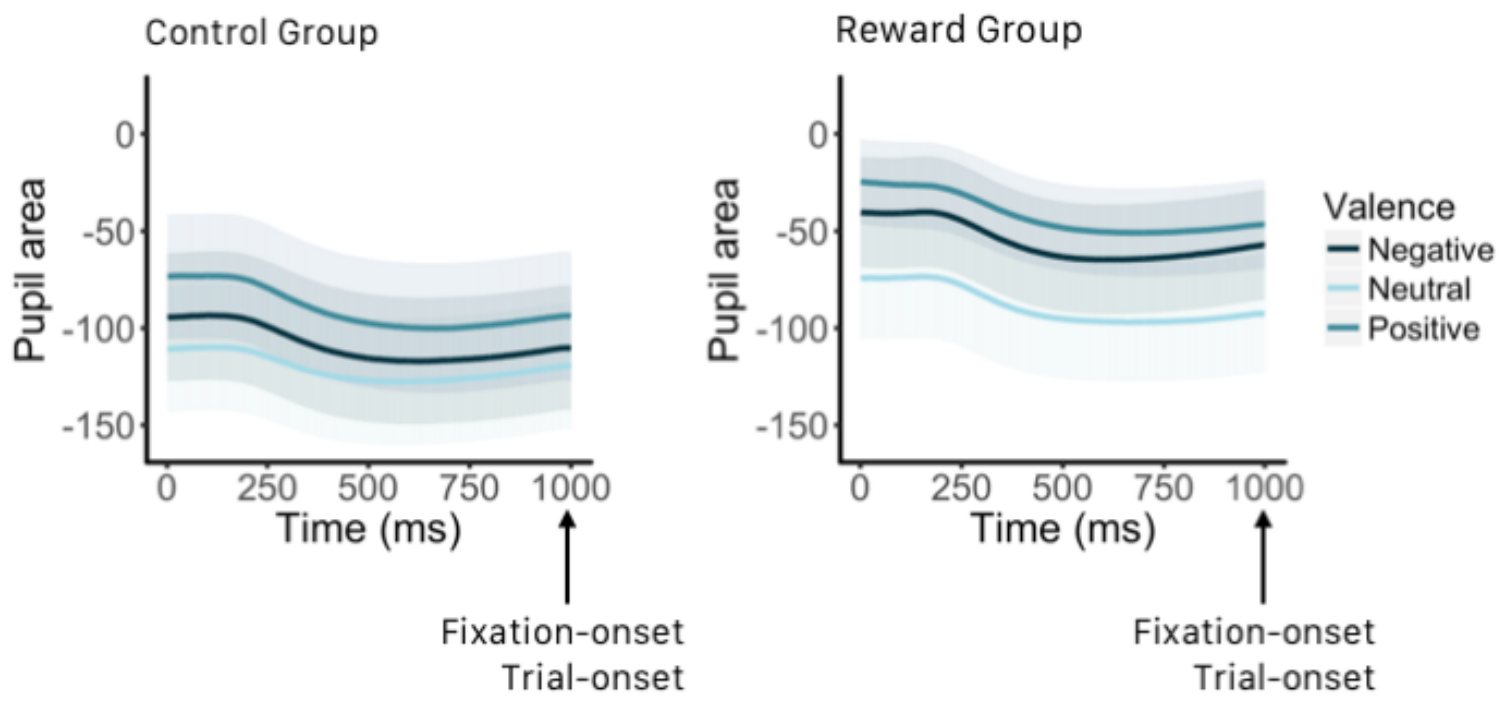

Figure 4. Mean pupil area (in arbitrary units) across the tonic time-window. Mean pupil area across the $1000 \mathrm{~ms}$ time-window preceding trial-onset, subtraction-baselined to the mean during the $200 \mathrm{~ms}$ time-window immediately preceding trial-onset in the baseline (scrambled-image only, non-reward) block. Overall, there was greater tonic dilation (or, more accurately, less constriction relative to baseline) on emotional compared to neutral blocks (with no difference between tonic dilation on positive and negative blocks). Tonic dilation was not modulated by reward group. Shaded error bands represent the standard error.

Contrary to predictions, analysis of the phasic proactive control indices (reflecting preparation during the $2000 \mathrm{~ms}$ fixation period preceding stimulus-onset; Figure 5A) revealed no main effect of reward group, $F(1,108)=0.29, p=.591, \eta_{p}{ }^{2}=0.003$, nor a reward group $\mathrm{x}$ valence interaction, $F(2,216)=0.29, p=.748, n_{p}{ }^{2}=0.003$ (quadratic interaction: $F(1,108)=$ $\left.0.51, p=.476, \eta_{p}{ }^{2}=0.005\right)$. Also contrary to predictions, there was no effect of valence on phasic proactive control, $F(2,216)=0.70, p=.499, \eta_{p}{ }^{2}=0.006$ (quadratic: $F(1,108)=0.09$, $\left.p=.766, \eta_{p}{ }^{2}=0.001\right)$. Here we see no evidence that participants dynamically prepare differently for an upcoming trial as a function of either reward group or valence of a potential distractor.

Post-stimulus pupil indices. An ANOVA was conducted on early and late poststimulus pupil indices (which reflect intact distractor processing relative to scrambled distractor processing) with valence (negative, neutral, positive) and time (early, late) as the 
Motivation and Emotional Distraction

within-subjects factors and reward group (reward, controls) as the between-subjects factor.

We had no specific predictions regarding time. See Table 3 for means for each group, split by valence and time, and Figure 6A for mean pupil area across the post-stimulus time-window.

Table 3. Comparisons of mean pupil area for the preregistered post-stimulus indices, for scrambled versus intact images, for each distractor-valence condition, separately for control and reward groups.

\begin{tabular}{|c|c|c|c|}
\hline \multirow{2}{*}{$\begin{array}{l}\text { Valence } \\
\text { Block }\end{array}$} & \multirow{2}{*}{$\begin{array}{l}\text { Scrambled } \\
\text { Trials }\end{array}$} & \multirow{2}{*}{$\begin{array}{l}\text { Intact } \\
\text { Trials }\end{array}$} & \multirow{2}{*}{ Difference } \\
\hline & & & \\
\hline \multicolumn{4}{|c|}{ Control Group } \\
\hline \multicolumn{4}{|l|}{ Early } \\
\hline Negative & $67(57)$ & $116(85)$ & $49(50)$ \\
\hline Neutral & $67(57)$ & $79(65)$ & $12(33)$ \\
\hline Positive & $72(66)$ & $155(89)$ & $83(54)$ \\
\hline \multicolumn{4}{|l|}{ Late } \\
\hline Negative & $14(59)$ & $55(79)$ & $41(59)$ \\
\hline Neutral & $25(71)$ & $27(77)$ & $2(51)$ \\
\hline Positive & $26(71)$ & $101(82)$ & $75(64)$ \\
\hline \multicolumn{4}{|c|}{$\underline{\text { Reward Group }}$} \\
\hline \multicolumn{4}{|l|}{ Early } \\
\hline Negative & $103(58)$ & $134(74)$ & $31(47)$ \\
\hline Neutral & $102(61)$ & $104(70)$ & $2(31)$ \\
\hline Positive & $106(63)$ & $165(82)$ & $59(51)$ \\
\hline \multicolumn{4}{|l|}{$\underline{\text { Late }}$} \\
\hline Negative & $20(62)$ & $57(87)$ & $37(69)$ \\
\hline Neutral & $29(66)$ & $31(70)$ & $2(38)$ \\
\hline Positive & $25(79)$ & $83(93)$ & $58(67)$ \\
\hline
\end{tabular}

Note: Difference is [intact - scrambled]. SDs are in brackets. $n=55$ per group. 
Motivation and Emotional Distraction

The predicted quadratic main effect of valence indicated that emotional distractors produced greater pupil dilation than neutral distractors, $F(1,108)=108.50, p<.001, \eta_{p}{ }^{2}=$ 0.501. Dilation in response to positive distractors $(M=69, S D=57)$ was greater than for negative distractors $(M=40, S D=53), t(109)=5.00, p<.001, d=0.476,95 \% \mathrm{CI}[17,40]$ (two-tailed), so these were separately compared to neutral post-stimulus pupil dilation (onetailed). Pupil dilation following positive, $t(109)=11.39, p<.001, d=1.086,95 \%$ CI [55, inf], and negative, $t(109)=6.40, p<.001, d=0.610,95 \% \mathrm{CI}[26$, inf], distractors was greater than pupil dilation following neutral distractors $(M=4, S D=36)$. The predicted reward group x valence interaction was not significant, $F(2,216)=0.98, p=.378, \eta_{p}^{2}=.009$, (quadratic interaction: $F(1,108)=1.18, p=.280, \eta_{p}{ }^{2}=.011$ ), suggesting that (unlike for behavioural distraction) reward did not reduce pupil dilation in response to emotional distractors to a greater extent than neutral distractors.

A trend toward the predicted main effect of reward group on post-stimulus pupil dilation, $F(1,108)=3.03, p=.084, \eta_{p}{ }^{2}=0.027$, was qualified by a time $\mathrm{x}$ reward group interaction (which was not predicted), $F(1,108)=4.16, p=.044, \eta_{p}{ }^{2}=0.037$. To explore this interaction, two ANOVAs were conducted with valence as the within-subjects factor and reward group as the between-subjects factor, separately for the early and late time-windows. This showed a significant effect of reward group on post-stimulus pupil dilation in the early time window, $F(1,108)=6.93, p=.010, \eta_{p}{ }^{2}=0.060$, but not in the late time-window, $F(1$, $108)=0.74, p=.392, \eta_{p}{ }^{2}=.007$. The timecourse of the effect of reward group on poststimulus pupil dilation is further followed up in exploratory functional data analyses, below.

Exploratory functional data analyses. The following functional data analyses (FDA) are exploratory. Averaging pupil dilation across pre (or post) specified (albeit arbitrary) time-windows is common in pupillometry studies (e.g., Bradley et al., 2008; Chiew \& Braver, 2013, 2014; Chevalier et al., 2015; Cohen et al., 2015; Henderson et al., 2014; Rondeel et al., 2015) and we chose to use indices calculated from pupil area in preregistered time-windows in keeping with these practices. However, in reducing the rich $(1000 \mathrm{~Hz})$ pupil data to mean dilation across long windows, we lose sensitivity to detect potential group differences in the timecourse of the implementation of control (see Sirois \& Brisson, 2014 for discussion of this issue). FDA (see Ramsay \& Silverman, 1997; 2005) solves this problem; the discrete pupil samples are converted into smooth functions, and then inferential statistics are performed on the functions over time. The output of a functional $t$-test is a smooth function that defines the critical $t$-value (and associated $p$ value) at each time point; the advantage of this approach is that we can identify precisely if and when there are significant 
Motivation and Emotional Distraction

group differences (see Brisson et al., 2013; Jackson \& Sirois, 2009 for use of FDA with pupillometry data). We conducted FDA only on the phasic pre-stimulus response and the post-stimulus response, as these windows capture the dynamic changes in pupil dilation related to stimulus events. FDA is less appropriate for analyzing tonic dilation, where the goal is to identify stable difference in overall levels of dilation. Because the analysis was exploratory, we did not restrict it to the preregistered windows, and so FDA was applied to two time-windows, the first across the entire $2000 \mathrm{~ms}$ pre-stimulus window (from fixation onset to stimulus-onset); and the second across the entire $4100 \mathrm{~ms}$ post-stimulus-onset window (from stimulus-onset until the end of the ITI).

Following methods described by Ramsay \& Silverman (1997; 2005), cubic B-splines were created for each participant, for each valence, for each time-window, with a knot at each millisecond, and were smoothed by penalizing the second derivative. This creates a smooth curve that captures each participant's pupil response across time. The B-splines for individual participants were then collapsed across valence, and functional $t$-tests were conducted to examine the timecourse of group differences. To calculate the critical value of the test statistic, the labels of individual curves (for each participant, for each condition) were randomly shuffled and then the test statistic was calculated with the new labels. One thousand permutations were run, resulting in a null distribution (the pointwise critical value over time) based on the actual data. See OSF for plots of the functional $t$-test output.

Exploratory pupil proactive control functional t-tests. Visual inspection of the pupil data suggests that by averaging pupil dilation across the $2000 \mathrm{~ms}$ time-window prior to stimulus-onset (our preregistered index of phasic proactive control) we missed important information about how proactive control unfolds across time, under conditions of reward relative to no reward (see Figure 5A). Specifically, it appears that averaging across the timewindow might have disguised proactive control in the reward group that occurs close in time to stimulus-onset. To address this possibility, we conducted a functional $t$-test to determine whether (and when) this apparent group difference is significant. The timing of significant group differences are marked by asterisks on Figure 5B. A significant group difference begins around $500 \mathrm{~ms}$ into the pre-stimulus period, extending until $1300 \mathrm{~ms}$ into the prestimulus period. However, contrary to predictions, this early difference is due to increased pupil dilation in the control group relative to the reward group, suggesting that the control group is implementing greater control than the reward group early in the fixation period. The second group difference occurs later, beginning around $1750 \mathrm{~ms}$ (250 ms prior to stimulusonset) and extends until stimulus-onset. This difference is in line with predictions, with the 
Motivation and Emotional Distraction

reward group showing greater pupil dilation, reflecting enhanced proactive control. Indeed, given that the reward group showed less dilation than the control group early in the pre-trial window, their eventual greater dilation reflects a steep gradient in dilation just before stimulus onset. The functional $t$-test reveals that the control and reward groups prepared to ignore the distractors over different timecourses. Specifically, the control group exerted effort earlier, and less intensely, with a small boost of control close to stimulus-onset. In contrast, the reward group prepared later, but more intensely, with a large dynamic boost of control close in time to stimulus-onset.

\section{A) Pre-stimulus Phasic}

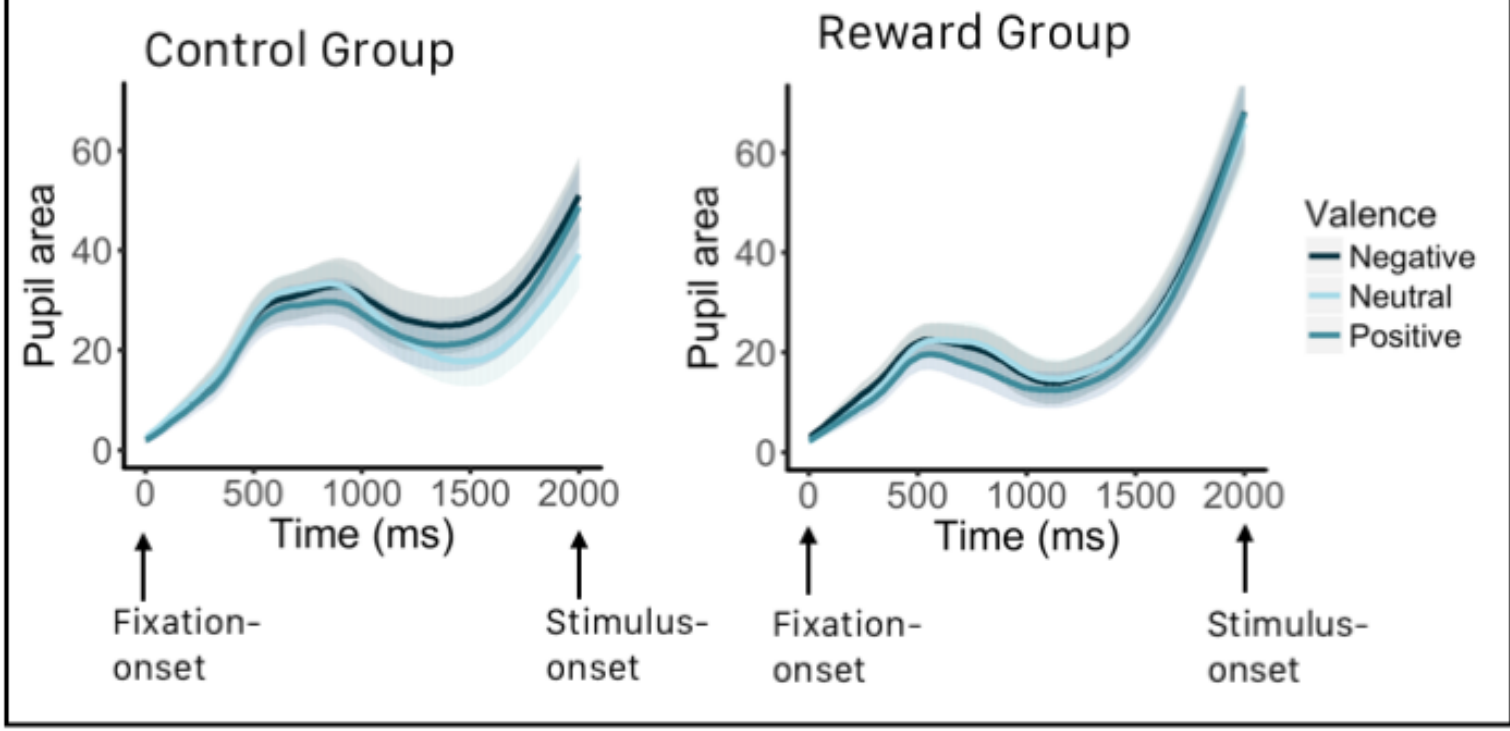

B) Pre-stimulus Phasic

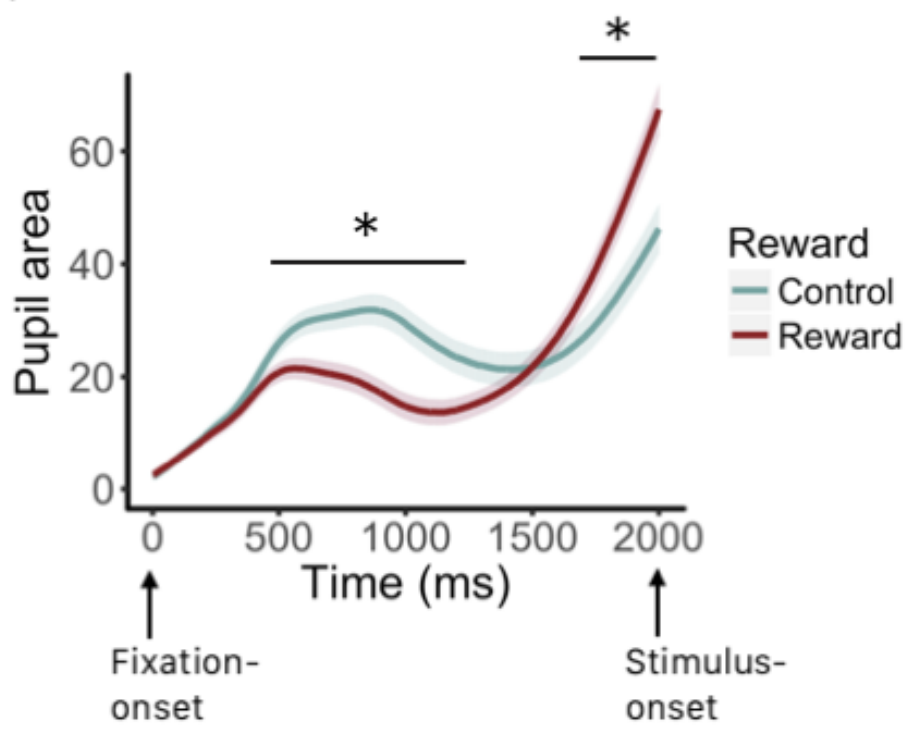


Figure 5: Mean pupil area (arbitrary units) across the pre-stimulus phasic time-window. Panel A: Phasic proactive control is indexed by mean pupil area during the $2000 \mathrm{~ms}$ timewindow preceding stimulus-onset, subtraction-baselined to the mean during the $200 \mathrm{~ms}$ timewindow immediately preceding trial-onset. The preregistered analysis indicated no modulation of the mean phasic proactive control index (collapsed across the entire prestimulus time-window) by either distractor valence or reward group. Panel B: Phasic pupil dilation collapsed by valence during the pre-stimulus $2000 \mathrm{~ms}$ time-window. Significant group differences from the functional t-test are indicated by the horizontal lines and asterisks. Shaded error bands represent the standard error.

Exploratory post-stimulus pupil functional t-tests. In the preregistered post-stimulus pupil ANOVA we found a trend toward the predicted main effect of reward group on poststimulus pupil dilation, which was qualified by a time $\mathrm{x}$ reward group interaction. This interaction suggests an effect of reward group on pupil dilation elicited by the intact (relative to scrambled) distractors during some but not all of the post-stimulus time-period. To discover precisely when this group difference was significant, a functional $t$-test was performed on the [intact - scrambled] difference in pupil area across the $4100 \mathrm{~ms}$ timewindow, beginning at stimulus-onset and extending to the end of the ITI period (subtractionbaselined to the $200 \mathrm{~ms}$ time-window immediately preceding stimulus-onset) comparing pupil area between the reward and control groups. This time-window encompasses both the early and late preregistered post-stimulus phasic pupil indices, as well as the $200 \mathrm{~ms}$ stimulus-presentation and the $300 \mathrm{~ms}$ post stimulus-offset periods which were not included in the preregistered time-windows. The timing of significant group differences (extending from around 1000 to $2400 \mathrm{~ms}$ post-stimulus) are marked on Figure 6B. 
Motivation and Emotional Distraction

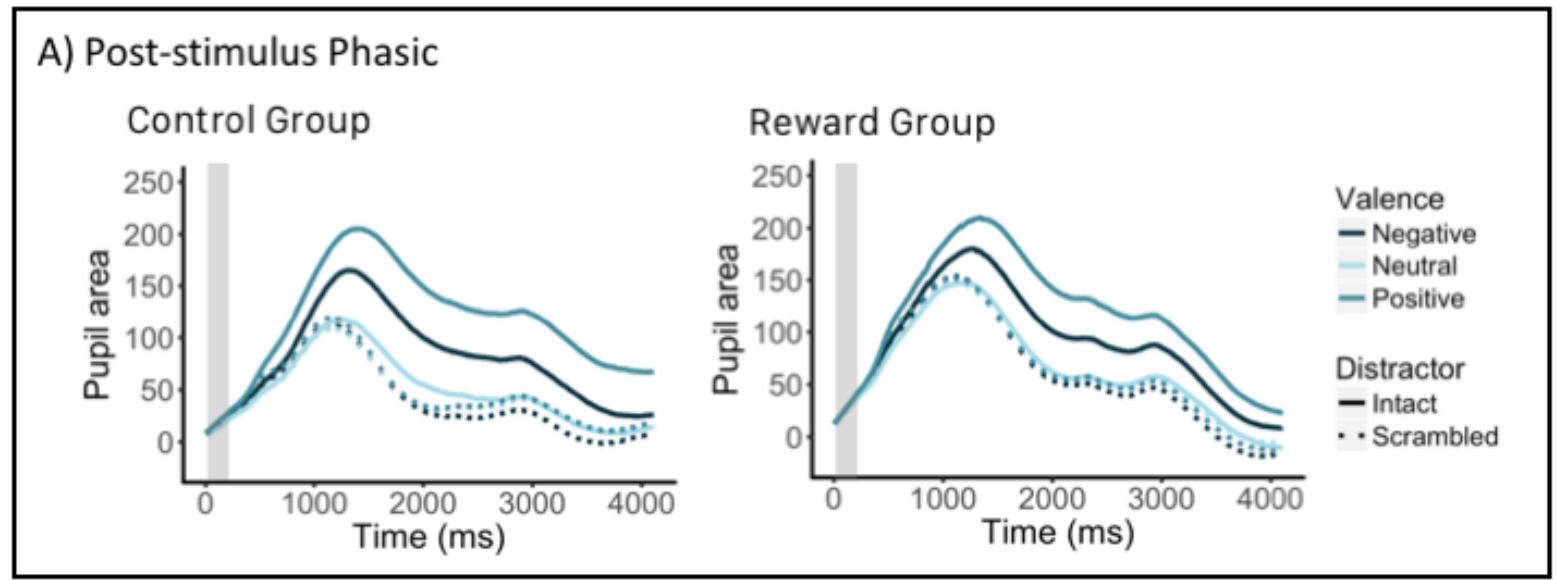

B) Post-stimulus Phasic

Intact and Scrambled trials

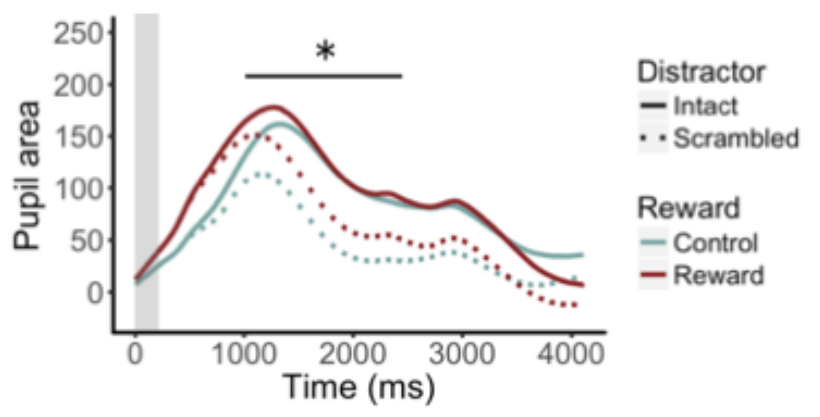

C) Post-stimulus Phasic

Intact trials

Scrambled trials
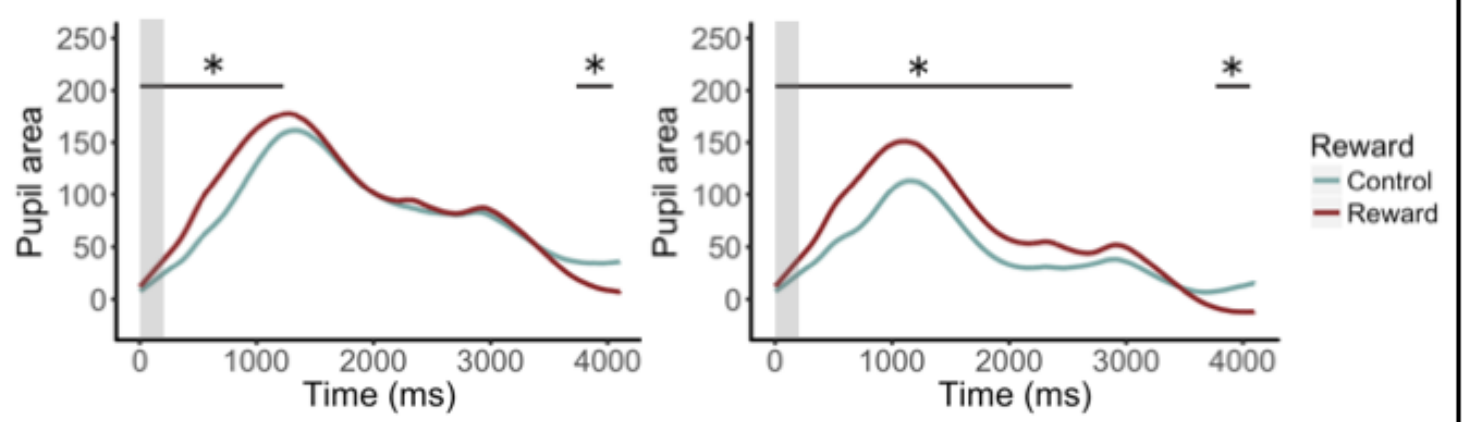

Figure 6: Mean pupil area (arbitrary units) across the 4100 ms post-stimulus time-window. All graphs show pupil area across the entire post-stimulus period starting from stimulusonset (subtraction-baselined to the mean $200 \mathrm{~ms}$ prior to stimulus-onset). Panel A: Pupil area split by valence, distractor type, and group (left: control group, right: reward group). Intact trials are represented by solid lines; scrambled images are represented by dotted lines. Distractor valence is represented by the different colour shades. Preregistered analyses of pupil indices from early and late post-stimulus time-windows showed modulation by both valence and reward group (see post-stimulus pupil indices). Panel B: Pupil dilation, collapsed across valence, split by distractor type and reward group. Intact trials are represented by solid lines, scrambled images are represented by dotted lines. Reward group is represented by the different colours. Significant group differences for the [intact scrambled] difference from the functional t-test are indicated by the horizontal lines and 
Motivation and Emotional Distraction

asterisks. Panel C: Pupil dilation on intact trials (left), and scrambled trials (right), split by reward group. Significant group differences from the functional t-test are indicated by the horizontal lines and asterisks.

Inspection of Figure 6A suggests that, in addition to a reduction in the [intact scrambled] difference in pupil dilation, there appears to be a shift toward greater pupil dilation in the reward group relative to the control group. To discover precisely when this group difference is significant, two functional $t$-tests were performed comparing the poststimulus pupil dilation (separately for intact and scrambled trials, collapsed across valence) over the entire $4100 \mathrm{~ms}$ post-stimulus time-window, to compare differences between the reward and control groups. For intact trials, there was greater pupil dilation in the reward group than in the control group for the first $1200 \mathrm{~ms}$ of the post-stimulus time-window (marked on Figure 6C, left graph). For scrambled trials, there was greater pupil dilation in the reward than the control group for the first $2550 \mathrm{~ms}$ of the post-stimulus time-window (marked on Figure 6C, right graph). Thus, while reward decreased pupil responses to intact relative to scrambled images (suggesting a reduction in sensitivity to the content of images), it did so by increasing responses to scrambles to a greater extent than to intact images in the early time window. We return to the implications of this finding in the Discussion.

Exploratory pupil-behaviour correlations. Nine exploratory correlations (see Figure 7) were calculated to examine how pupil dilation relates to behavioural responses. We examined the relationship between each mean pupil index (tonic, phasic pre-stimulus, phasic post-stimulus) and behavioural distraction indices, separately for each valence block (positive, negative, neutral). All significant correlations survived a Bonferroni correction (at alpha $=.05$, adjusted to .006 for nine tests) unless noted otherwise. First, to determine whether the phasic post-stimulus [intact - scrambled] difference in pupil dilation does actually reflect processing of the emotional distractors, we examined correlations between phasic post-stimulus dilation (averaged across the early and late time-windows) and behavioural distraction. Positive correlations were significant for all three valences: negative blocks, $r(108)=.396, p<.001$; positive blocks, $r(108)=.296, p=.002$; and neutral blocks, $r(108)=.232, p=.015$. Note however that the correlation for neutral blocks does not survive the Bonferonni correction (corrected $p=.135$ ). These findings validate the post-stimulus pupil dilation index as a measure of processing of the content of the emotional images, showing that the greater the pupil dilation following intact relative to scrambled images, the greater the behavioural distraction. 
Motivation and Emotional Distraction

The tonic pupil index correlated negatively with emotional behavioural distraction, with greater tonic dilation on negative and positive blocks being associated with less negative, $r(108)=-.265, p=.005$, and positive, $r(108)=-.259, p=.006$, behavioural distraction, respectively. The correlation between tonic pupil dilation and behavioural distraction on positive blocks became marginal with the Bonferonni correction $(p=.054)$. Tonic dilation on neutral blocks did not correlate with behavioural distraction, $r(108)=-.144$, $p=.134$. These findings are consistent with the interpretation that increased tonic dilation is associated with increased sustained control when distractors are expected to be emotional.

However, phasic proactive pupil dilation (collapsed across the $2000 \mathrm{~ms}$ pre-stimulus time-window) did not correlate with negative, $r(108)=.042, p=.664$, positive, $r(108)=.099$, $p=.302$, or neutral, $r(108)=-.053, p=.584$, behavioural distraction ${ }^{10}$.

\footnotetext{
${ }^{10}$ Additional exploratory correlations were calculated between pupil dilation in the time windows that were sensitive to reward in the functional analyses $(500-1300 \mathrm{~ms}$ and $1750-2000 \mathrm{~ms}$ in the pre-stimulus timewindow) and behavioural distraction. These were calculated separately for the control and reward groups, and for each valence. None of these correlations reached significance (all $p$ 's $>.107$, uncorrected for multiple comparisons).
} 
Motivation and Emotional Distraction
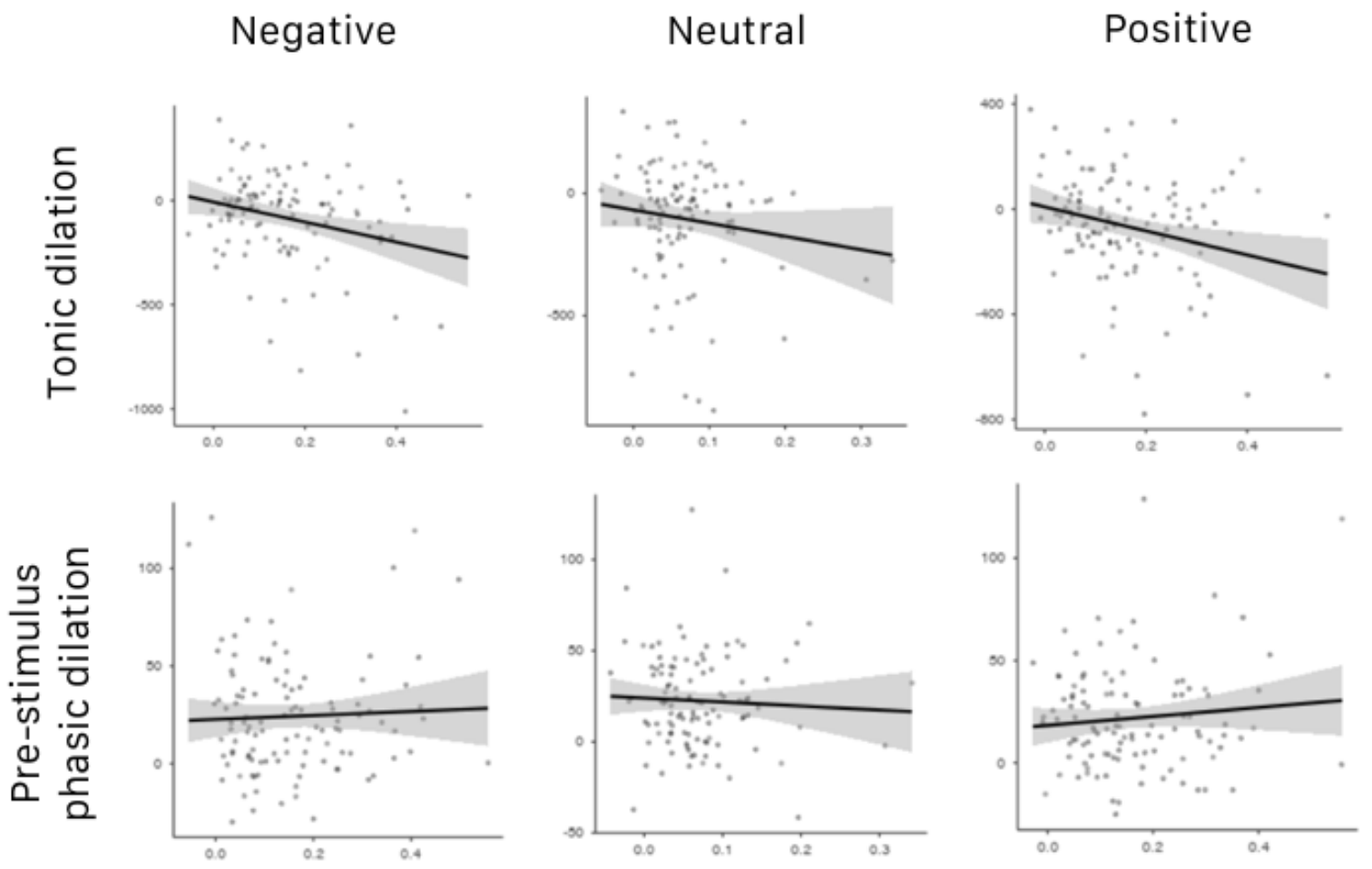

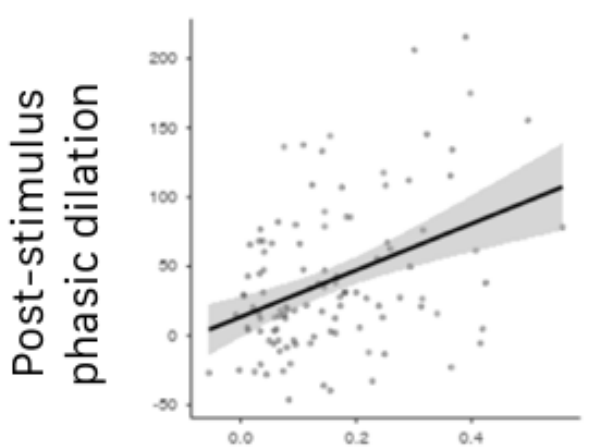

Distraction indices (\%)

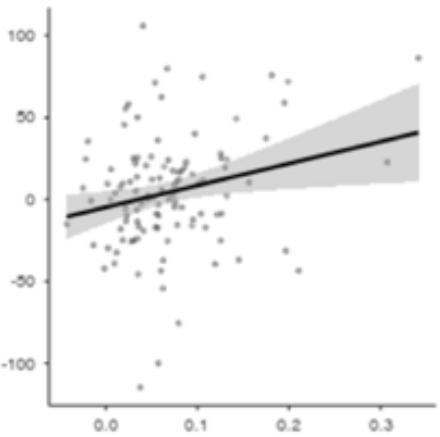

Distraction indices (\%)

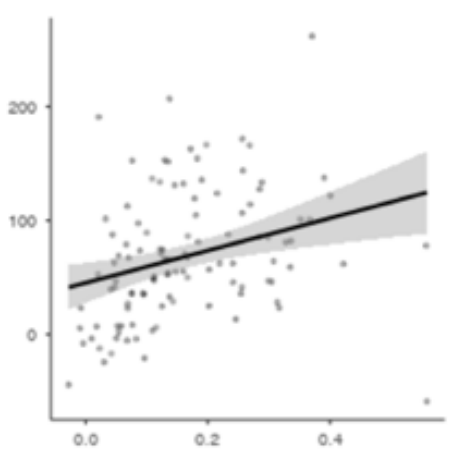

Distraction indices (\%)

Figure 7: Pupil indices - behavioural distraction correlations. Correlations between tonic (top row), pre-stimulus phasic (middle row), and post-stimulus phasic (bottom row) mean pupil indices and behavioural distraction indices on negative (left column), neutral (middle column) and positive (right column) blocks. Significant positive correlations were found for post-stimulus phasic pupil dilation (intact-scrambled difference) and behavioural distraction on positive and negative blocks. Significant negative correlations were found for tonic dilation and behavioural distraction for positive and negative blocks.

\section{Discussion}

As predicted, the availability of performance-contingent reward decreased distraction by irrelevant images, particularly those that were emotional. These behavioural findings replicate our previous findings using a similar paradigm (Walsh et al., 2018), and are consistent with a growing body of evidence that motivation enhances cognitive control, even when distractors are highly emotional (Padmala \& Pessoa, 2014; Padmala, et al., 2017). 


\section{Motivation and Emotional Distraction}

Mirroring the behavioural findings, and relative to scrambled control stimuli, emotional distractors produced greater dilation than neutral distractors; and dilation to distractors (relative to scrambled control images) was reduced by reward. Furthermore, greater poststimulus dilation to emotional distractors predicted greater behavioural emotional distraction, establishing pupil dilation as a valid measure of emotional processing that has behavioural consequences.

Our primary research question concerned the timecourse by which motivation reduces distraction. Specifically, we tested the hypothesis that reward encourages a shift to an effective proactive control strategy to ignore emotional distractors. We considered two variants of proactive control indexed by pupil dilation: dynamic up-regulation of control in preparation for an imminent distractor; and sustained implementation of control across entire blocks. Although we found no evidence that reward modulated sustained proactive control, exploratory analyses showed dynamic implementation of proactive control close in time to stimulus-onset in the reward group. Exploratory analyses also revealed that reward increased post-stimulus dilation (regardless of distractor type), suggesting up-regulation of a reactive control mechanism. Together, these findings (examined in more detail below) suggest dynamic implementation of anticipatory control to more effectively disengage from distractors. We found no evidence that reward influences the timecourse of control differently for positive, negative, or neutral distractions.

We tested hypotheses using preregistered pupil indices (analysing baselined mean pupil area in specified time-windows) and exploratory analyses using a more data-driven approach to identify time periods in which pupil dilation was sensitive to reward. The tonic proactive control index (mean pupil area across the $1000 \mathrm{~ms}$ period prior to trial-onset) was greater in blocks in which distractors were emotional. It is difficult to determine whether this effect indicates greater sustained anticipatory control when emotional distractors were expected, or whether it is the result of emotional arousal carrying over from previously presented emotional distractors (which will have occurred on $25 \%$ of trials). One way to assess carryover would be to analyse trials that followed an intact distractor separately from those that followed a scramble; however, we did not have sufficient trial numbers to make such an analysis robust. The correlations with behaviour, however, argue against emotional carryover, because greater tonic dilation on emotional blocks was associated with less emotional distraction, consistent with the conclusion that tonic dilation is associated with enhanced sustained proactive control of emotional distraction. Emotional carryover, in 
Motivation and Emotional Distraction

contrast, would predict a positive relationship between tonic dilation and distraction on emotional blocks.

Importantly, the tonic proactive control pupil index was not affected by reward, nor did reward interact with valence. Findings therefore do not support the hypothesis that the availability of reward encourages the use of a sustained proactive control strategy in this task. Our findings contrast with those of Chiew and Braver $(2013,2014)$, who found that reward enhanced both tonic pupil dilation and behavioural proactive control. Our null effect of reward on sustained proactive control could be due to insufficient power; tonic pupil data has high variability, and numerically our reward group did show higher tonic dilation than controls. However, the significant effect of emotion on tonic dilation (greater tonic dilation in emotional compared to neutral blocks) suggests that our study did not lack power to detect cognitive effects on tonic dilation.

The preregistered phasic proactive control pupil index (mean pupil area across the $2000 \mathrm{~ms}$ pre-stimulus window) was not modulated by valence, suggesting that similar dynamic proactive mechanisms were engaged preceding emotional and neutral distractions. Preregistered analyses also revealed no influence of reward on phasic proactive control. However, the exploratory functional $t$-test showed a significant effect of reward, which arose because the timecourse of preparatory phasic pupil dilation differed between reward conditions. The test revealed (see Figure 5B) an early window (from around 500-1300 ms post fixation-onset) in which pupil dilation was greater in control than in reward participants, and a late window (extending from around $1750 \mathrm{~ms}$ after fixation-onset and continuing until stimulus-onset) in which phasic pupil dilation was greater (and rapidly increasing) in reward than in control participants. It appears that the control group exerted control earlier and less intensely than the reward group, who exerted a sharp "burst" of proactive control right before onset of the anticipated stimulus. This dynamic effect of reward on phasic proactive control is consistent with the effect of reward in non-emotional contexts (Chiew \& Braver, 2013, 2014), and indeed reward did not interact with valence in any of our pre-stimulus analyses.

Turning to the post-stimulus period, pupil dilation was modulated by both emotion and reward, in both the preregistered and exploratory analyses. Our preregistered analyses used indices of phasic post-stimulus pupil dilation (mean pupil response to intact distractors relative to scrambles), to assess processing of the content of distractor images while controlling for low-level visual features. Although behavioural distraction and the pupil 


\section{Motivation and Emotional Distraction}

response to distractors were broadly similar (both were greater for emotional than for neutral distractors, and both were reduced by reward); there were some inconsistencies in the effects of valence. Pupil dilation was greater following positive (erotic) distractors than following negative (mutilation) distractors, which both produced greater dilation than neutral distractors. In contrast, behavioural distraction from positive and negative images did not differ (see also Walsh et al., 2018). The valence effect in the pupil response is consistent with findings from Henderson and colleagues (2014) showing greater pupil dilation to erotic images relative to negative images, even when they are matched for subjective ratings of arousal.

Behaviourally, reward produced greater attenuation of distraction in emotional blocks than in neutral blocks. This behavioural effect is consistent with the notion that, in addition to generally enhancing cognitive control (as reward does in emotionally-neutral contexts), reward may reduce emotional distraction by additionally increasing the motivational value of the targets. If we attend to emotional distractors partly because we are motivated to attend to them, then enhancing the motivational value of targets might influence competition between targets and emotional distractors to a greater degree than between targets and neutral distractors (which lack this motivational value). However, this effect was not observed in the pupil index, which showed similar effects of reward on responses to emotional and neutral distractors.

Interestingly, further exploratory functional data analyses (FDA) showed that the effect of reward on post-stimulus dilation was driven primarily by greater dilation on trials with scrambled distractors (but to a lesser extent intact distractors) in rewarded participants compared to controls. This suggests a general, control-driven increase in dilation under reward. Separately examining the timecourse of group differences in dilation on intact trials, scrambled trials, and the [intact - scrambled] difference, allows us to make inferences regarding the respective influences of reward on control- and emotion-related pupil dilation. Reward was associated with greater pupil dilation for both scrambles and intact images (regardless of valence) right from stimulus onset (Figure 6C). However, the effect of reward on the intact-scrambled difference did not emerge until about $1000 \mathrm{~ms}$ later (Figure 6B). We suggest that the early effect of reward reflects up-regulation of reactive cognitive control that is implemented in the face of any distractor. The later effect of reward reflects reduced emotional processing of distractors, due to more rapid implementation of control. The idea that there are separable cognitive and emotional influences on pupil dilation is not new. 


\section{Motivation and Emotional Distraction}

Dilation due to emotional arousal is thought to be under sympathetic control (Beatty \& Lucero-Wagoner, 2000; Bradley et al., 2008), activating the pupil's dilator muscle; whereas dilation due to cognitive effort is the result of parasympathetic inhibition of the constrictor muscle (Samuels \& Szabadi, 2008). Thus it is possible for cognitive control and emotional arousal to independently affect pupil size.

A similar dissociation between control-related and emotion-related pupil responses was recently described by Kinner and colleagues (2017). They observed pupil responses to neutral and negative images that participants viewed passively, or that they prepared to view under instruction to either up-regulate or down-regulate their emotional response. Pupil dilation in an early time-window after image onset was enhanced in emotion-regulation conditions, regardless of whether the instruction required them to up-regulate or downregulate their emotional response. It was not until a later time-window that responses diverged, becoming larger in the up-regulation than in the down-regulation condition. Kinner and colleagues argue that early pupil responses reflect a "cognitive" component, indexing the effort applied to both emotion regulation strategies, whereas later responses reflect an "emotional" component that indexes the emotional consequences of that regulation. As described above, our findings could be similarly interpreted as showing an early effect of reward on pupil dilation, independent of the nature of the distractor, which reflects effortful control; followed by a later effect of reward on pupil dilation, dependent on distractor content, which reflects an emotional response. Considering that (in our data) reward enhances control-related dilation right from stimulus-onset, this dilation may reflect a continuation of anticipatory control processes, or very fast activation of reactive control processes (e.g., disengagement), which continue long after stimulus-offset and even behavioural response. Indeed, we can anticipatorily prepare to disengage from distractors (rather than proactively preventing their attentional selection; Theeuwes, 2010). Future research utilising other temporally-sensitive measures, such as electroencephalography, may yield further insights into the timecourse of reward-based modulation of control of emotional distraction.

Although a decomposition of the pupil response into a cognitive and an emotional component provides a parsimonious explanation of our data, we cannot rule out an alternative explanation: that anticipation of reward induces emotional arousal that is reflected in the pupil response (e.g., Bouret \& Richmond, 2015). At this point we can say that post-stimulus pupil responses were separately modulated by both reward and the emotional nature of the 
Motivation and Emotional Distraction

distractor. Manipulations that can tease apart the relative contributions of control and emotion on pupil dilation will be an important goal for future studies. We also note that analysis of the post-stimulus pupil response in the late time-window could be influenced by performance feedback (presented 2000 ms post stimulus-onset), which should be more affectively positive in the reward group than in the control group. However, group differences in the poststimulus pupil response (revealed by both the preregistered analyses and the exploratory FDA) were almost exclusively limited to the time-window prior to feedback presentation, and therefore the potential affective influence of feedback does not impact our findings and conclusions.

Limitations in this study must be acknowledged. First, our findings in support of dynamic changes in proactive control were observed in the exploratory FDA, and not in our preregistered analyses using pre-defined windows. These exploratory findings therefore require replication with a preregistered analysis plan. Second, we limited our stimulus set to high arousal negative (mutilation) and positive (erotic) images, because they are known to produce robust pupil responses. Extension to other stimulus categories, perhaps with different motivational value, will be necessary to determine how well these effects generalise. Third, our erotic image set included only heterosexual couples, and we did not ask participants about their sexual orientation. The subjective emotional value of both erotic and mutilation images naturally differs across people, with sexual orientation being one influencing factor. Although random assignment to reward groups should mean these individual differences will not account for our experimental effects, such variability might be useful to explore in future work. Finally, our neutral images were matched with the emotional images on content, and therefore contained people. The use of "interesting" neutral images may have masked potential reward by emotion interactions that may emerge with the use of more mundane neutral distractors (e.g., household objects).

Attending to emotional stimuli is sometimes crucial for survival. But when emotional stimuli are neither imperative, nor relevant to current goals, we face the challenge of ignoring them. The current study sits at the intersection of the growing, but largely separate, literatures on the effect of reward on cognitive control in emotionally-neutral contexts on the one hand; and the effect of (non-reward) control manipulations on emotional distraction on the other. Our findings confirm that performance-contingent reward can reduce emotional distraction, and suggest that it does so by dynamically up-regulating control just before the anticipated onset of a stimulus, and extending that control into the post-stimulus period. We also show 
Motivation and Emotional Distraction

the value of pupillometry - a non-invasive and relatively low-cost tool - to further understanding of both emotion and cognitive control. 
Motivation and Emotional Distraction

References

Armstrong, R. A. (2014). When to use the Bonferroni correction. Ophthalmic and Physiological Optics, 34(5), 502-508. doi: 10.1111/opo.12131

Beatty, J., \& Lucero-Wagoner, B. (2000). The pupillary system. Handbook of psychophysiology, 2(142-162).

Botvinick, M., \& Braver, T. (2015). Motivation and cognitive control: from behaviour to neural mechanism. Annual Review of Psychology, 66(1), 83-113. doi:10.1146/annurev-psych-010814-015044

Bouret, S., \& Richmond, B. J. (2015). Sensitivity of locus coeruleus neurons to reward value for goal-directed actions. Journal of Neuroscience, 35(9), 4005-4014. doi: 10.1523/JNEUROSCI.4553-14.2015

Bradley, M. M., Miccoli, L., Escrig, M. A., \& Lang, P. J. (2008). The pupil as a measure of emotional arousal and autonomic activation. Psychophysiology, 45(4), 602-607. doi: 10.1111/j.1469-8986.2008.00654.x

Braver, T. S. (2012). The variable nature of cognitive control: a dual mechanisms framework. Trends in Cognitive Sciences, 16(2), 106-113. doi: 10.1016/j.tics.2011.12.010

Braver, T. S., Gray, J. R., \& Burgess, G. C. (2007). Explaining the many varieties of working memory variation: Dual mechanisms of cognitive control. In A. R. A. Conway, C.

Jarrold, M. J. Kane, A. Miyake \& J. N. Towse (Eds.), Variation in working memory (pp.76-106). Oxford: Oxford University Press.

Braver, T. S., Paxton, J. L., Locke, H. S., \& Barch, D. M. (2009). Flexible neural mechanisms of cognitive control within human prefrontal cortex. Proceedings of the National Academy of Sciences, 106(18), 73517356. doi: 10.1073/pnas.0808187106

Brisson, J., Mainville, M., Mailloux, D., Beaulieu, C., Serres, J., \& Sirois, S. (2013). Pupil diameter measurement errors as a function of gaze direction in corneal reflection eyetrackers. Behaviour Research Methods, 45(4), 1322-1331. doi: 10.3758/s13428-013-0327-0

Brosch, T., Pourtois, G., Sander, D., \& Vuilleumier, P. (2011). Additive effects of emotional, endogenous, and exogenous attention: behavioural and electrophysiological evidence. Neuropsychologia, 49(7), 1779-1787. doi: 10.1016/j.neuropsychologia.2011.02.056

Bruyer, R., \& Brysbaert, M. (2011). Combining speed and accuracy in cognitive psychology: is the inverse efficiency score (IES) a better dependent variable than the mean reaction time (RT) and the percentage of errors (PE)?. Psychologica Belgica, 51(1), 5-13. doi:10.5334/pb-51-1-5

Bugg, J. M., \& Crump, M. J. (2012). In support of a distinction between voluntary and stimulus-driven control: A review of the literature on proportion congruent effects. Frontiers in Psychology, 3:367. doi:10.3389/fpsyg.2012.00367

Carver, C. S., \& White, T. L. (1994). Behavioural inhibition, behavioural activation, and affective responses to impending reward and punishment: the BIS/BAS scales. Journal of Personality and Social Psychology, 67(2), 319. doi: 10.1037/0022-3514.67.2.319

Chatham, C. H., Frank, M. J., \& Munakata, Y. (2009). Pupillometric and behavioural markers of a developmental shift in the temporal dynamics of cognitive control. Proceedings of the National Academy of Sciences, 106(14), 5529-5533. doi: 10.1073/pnas.0810002106 


\section{Motivation and Emotional Distraction}

Chevalier, N., Martis, S. B., Curran, T., \& Munakata, Y. (2015). Metacognitive processes in executive control development: The case of reactive and proactive control. Journal of Cognitive Neuroscience, 27(6), 11251136. doi: 10.1162/jocn_a_00782

Chiew, K. S., \& Braver, T. S. (2013). Temporal dynamics of motivation-cognitive control interactions revealed by high-resolution pupillometry. Frontiers in Psychology, 4:15. doi:10.3389/fpsyg.2013.00015

Chiew, K. S., \& Braver, T. S. (2014). Dissociable influences of reward motivation and positive emotion on cognitive control. Cognitive, Affective, \& Behavioural Neuroscience, 14(2), 509-529. doi: 10.3758/s13415$\underline{014-0280-0}$

Cohen, N., Moyal, N., \& Henik, A. (2015). Executive control suppresses pupillary responses to aversive stimuli. Biological Psychology, 112, 1-11. doi: 10.1016/j.biopsycho.2015.09.006

Coull, J. T., Büchel, C., Friston, K. J., \& Frith, C. D. (1999). Noradrenergically mediated plasticity in a human attentional neuronal network. Neuroimage, 10(6), 705-715. doi: 10.1006/nimg.1999.0513

Faul, F., Erdfelder, E., Lang, A.-G., \& Buchner, A. (2007). G*Power 3: A flexible statistical power analysis program for the social, behavioural, and biomedical sciences. Behaviour Research Methods, 39, 175-191. doi.org/10.3758/BF03193146

Fröber, K., \& Dreisbach, G. (2014). The differential influences of positive affect, random reward, and performance-contingent reward on cognitive control. Cognitive, Affective \& Behavioural Neuroscience, 14(2), 530-547. doi: 10.3758/s13415-014-0259-х

Fröber, K., \& Dreisbach, G. (2016). How performance (non-)contingent reward modulates cognitive control. Acta Psychologica, 168, 65-77. doi: 10.1016/j.actpsy.2016.04.008

Geng, J. J. (2014). Attentional mechanisms of distractor suppression. Current Directions in Psychological Science, 23(2), 147-153. doi:10.1177/0963721414525780

Grimshaw, G. M., Kranz, L. S., Carmel, D., Moody, R. E., \& Devue, C. (2018). Contrasting reactive and proactive control of emotional distraction. Emotion. doi:10.1037/emo000033

Gupta, R., Hur, Y. J., \& Lavie, N. (2016). Distracted by pleasure: Effects of positive versus negative valence on emotional capture under load. Emotion, 16(3), 328-337. doi:10.1037/emo0000112

Hefer, C., \& Dreisbach, G. (2016). The motivational modulation of proactive control in a modified version of the AX-continuous performance task: Evidence from cue-based and prime-based preparation. Motivation Science; Washington, 2(2), 116-134. doi: 10.1037/mot0000034

Heitz, R. P., Schrock, J. C., Payne, T. W., \& Engle, R. W. (2008). Effects of incentive on working memory capacity: Behavioural and pupillometric data. Psychophysiology, 45(1), 119-129. doi: 10.1111/j.14698986.2007.00605.x

Henderson, R. R., Bradley, M. M., \& Lang, P. J. (2014). Modulation of the initial light reflex during affective picture viewing. Psychophysiology, 51(9), 815-818. doi: 10.1111/psyp.12236

Jackson, I., \& Sirois, S. (2009). Infant cognition: going full factorial with pupil dilation. Developmental Science, 12(4), 670-679. doi: 10.1111/j.1467-7687.2008.00805.x

Jones, N. P., Siegle, G. J., \& Mandell, D. (2015). Motivational and emotional influences on cognitive control in depression: A pupillometry study. Cognitive, Affective, \& Behavioural Neuroscience, 15(2), 263-275. doi: $10.3758 / \mathrm{s} 13415-014-0323-6$ 


\section{Motivation and Emotional Distraction}

Joshi, S., Li, Y., Kalwani, R. M., \& Gold, J. I. (2016). Relationships between pupil diameter and neuronal activity in the locus coeruleus, colliculi, and cingulate cortex. Neuron, 89(1), 221-234. doi: 10.1016/j.neuron.2015.11.028

Kahneman, D. (1973). Attention and effort (Vol. 1063). Englewood Cliffs, NJ: Prentice-Hall. doi:

Kinner, V. L., Kuchinke, L., Dierolf, A. M., Merz, C. J., Otto, T., \& Wolf, O. T. (2017). What our eyes tell us about feelings: Tracking pupillary responses during emotion regulation processes. Psychophysiology, 54(4), 508-518. doi: 10.1111/psyp.12816

Laeng, B., Sirois, S., \& Gredebäck, G. (2012). Pupillometry: a window to the preconscious?. Perspectives on Psychological Science, 7(1), 18-27. doi: 10.1177/1745691611427305

Lang, P. J., Bradley, M. M., \& Cuthbert, B. N. (2008). International affective picture system (IAPS): Affective ratings of pictures and instruction manual. Technical Report A-8.

LeDoux, J. (2012). Rethinking the emotional brain. Neuron, 73(4), 653-676. doi: 10.1016/j.neuron.2012.02.004

Locke, H. S., \& Braver, T. S. (2008). Motivational influences on cognitive control: behaviour, brain activation, and individual differences. Cognitive, Affective, \& Behavioural Neuroscience, 8(1), 99-112. doi: doi.org/10.3758/CABN.8.1.99

Mathôt, S., Fabius, J., VanHeusden, E., \& Van der Stigchel, S. (2017). Safe and sensible baseline correction of pupil-size data. PeerJ PrePrints. doi: 10.7287/peerj.preprints.2725v1

Mathôt, S., \& Van der Stigchel, S. (2015). New light on the mind's eye: The pupillary light response as active vision. Current Directions in Psychological Science, 24(5), 374-378. doi: 10.1177/0963721415593725

Mohanty, A., \& Sussman, T. J. (2013). Top-down modulation of attention by emotion. Frontiers in Human Neuroscience, 7, 102. doi: 10.3389/fnhum.2013.00102

Mulckhuyse, M. (2018). The influence of emotional stimuli on the oculomotor system: A review of the literature. Cognitive, Affective, \& Behavioural Neuroscience, 1-15. doi: 10.3758/s13415-018-0590-8

Okon-Singer, H., Tzelgov, J., \& Henik, A. (2007). Distinguishing between automaticity and attention in the processing of emotionally significant stimuli. Emotion, 7(1), 147-157. doi:10.1037/1528-3542.7.1.147

Padmala, S., \& Pessoa, L. (2011). Reward reduces conflict by enhancing attentional control and biasing visual cortical processing. Journal of Cognitive Neuroscience, 23(11), 3419-3432. doi:10.1162/jocn_a_00011

Padmala, S., \& Pessoa, L. (2014). Motivation versus aversive processing during perception. Emotion, 14(3), 450-454. doi:10.1037/a0036112

Padmala, S., Sirbu, M., \& Pessoa, L. (2017). Potential reward reduces the adverse impact of negative distractor stimuli. Social Cognitive and Affective Neuroscience, 12(9), 1402-1413. doi: 10.1093/scan/nsx067

Pessoa, L. (2009). How do emotion and motivation direct executive control? Trends in Cognitive Sciences, 13(4), 160-166. doi: 10.1016/j.tics.2009.01.006

Pessoa, L., \& Engelmann, J. B. (2010). Embedding reward signals into perception and cognition. Frontiers in Neuroscience, 4, 17. doi: 10.3389/fnins.2010.00017

Pourtois, G., Schettino, A., \& Vuilleumier, P. (2013). Brain mechanisms for emotional influences on perception and attention: what is magic and what is not. Biological Psychology, 92(3), 492-512. doi:10.1016/j.biopsycho.2012.02.007

Psychology Software Tools, Inc. [E-Prime 2.0]. (2012). Retrieved from http://www.pstnet.com. 


\section{Motivation and Emotional Distraction}

Rajkowski, J., Majczynski, H., Clayton, E., \& Aston-Jones, G. (2004). Activation of monkey locus coeruleus neurons varies with difficulty and performance in a target detection task. Journal of Neurophysiology, 92(1), 361-371. doi: 10.1152/jn.00673.2003

Ramsay, J. O., \& Silverman, B. W. (1997). Functional Data Analysis. New York: Springer-Verlag.

Ramsay, J. O., \& Silverman, B. W. (2005). Springer series in statistics. In Functional data analysis. Springer.

Rondeel, E., Van Steenbergen, H., Holland, R., \& van Knippenberg, A. (2015). A closer look at cognitive control: differences in resource allocation during updating, inhibition and switching as revealed by pupillometry. Frontiers in Human Neuroscience, 9, 494. doi: 10.3389/fnhum.2015.00494

Samuels, E. R., \& Szabadi, E. (2008). Functional neuroanatomy of the noradrenergic locus coeruleus: its roles in the regulation of arousal and autonomic function part I: principles of functional organisation. Current Neuropharmacology, 6(3), 235-253.

Sara, S. J. (2009). The locus coeruleus and noradrenergic modulation of cognition. Nature Reviews Neuroscience, 10(3), 211. doi: 10.1038/nrn2573

Sirois, S., \& Brisson, J. (2014). Pupillometry. Wiley Interdisciplinary Reviews: Cognitive Science, 5(6), 679692. doi: $10.1002 /$ wes. 1323

Snowden, R. J., O'Farrell, K. R., Burley, D., Erichsen, J. T., Newton, N. V., \& Gray, N. S. (2016). The pupil's response to affective pictures: Role of image duration, habituation, and viewing mode. Psychophysiology, 53(8), 1217-1223. doi: 10.1111/psyp.12668

Sussman, T. J., Jin, J., \& Mohanty, A. (2016). Top-down and bottom-up factors in threat-related perception and attention in anxiety. Biological Psychology, 121, 160-172. doi: 10.1016/j.biopsycho.2016.08.006

Theeuwes, J. (2010). Top-down and bottom-up control of visual selection. ActaPpsychologica, 135(2), 77-99. doi: 10.1016/j.actpsy.2010.02.006

Vanderhasselt, M. A., Remue, J., Ng, K. K., \& De Raedt, R. (2014). The interplay between the anticipation and subsequent online processing of emotional stimuli as measured by pupillary dilatation: the role of cognitive reappraisal. Frontiers in Psychology, 5, 207. doi: 10.3389/fpsyg.2014.00207

Walsh, A. T., Carmel, D., Harper, D., \& Grimshaw, G. M. (2018). Motivation enhances control of positive and negative emotional distractions. Psychonomic Bulletin \& Review, 1-7. doi: 10.3758/s13423-017-1414-5

Willenbockel, V., Sadr, J., Fiset, D., Horne, G. O., Gosselin, F., \& Tanaka, J. W. (2010). Controlling low-level image properties: the SHINE toolbox. Behaviour Research Methods, 42(3), 671-684. doi:10.3758/BRM.42.3.671

Yamaguchi, M., \& Nishimura, A. (2018). Modulating proactive cognitive control by reward: differential anticipatory effects of performance-contingent and non-contingent rewards. Psychological Research, 1-17. doi: 10.1007/s00426-018-1027-2

Yiend, J. (2010). The effects of emotion on attention: A review of attentional processing of emotional information. Cognition and Emotion, 24(1), 3-47. doi: 10.1080/02699930903205698 
Motivation and Emotional Distraction

Appendix D. Open Science Framework Preregistration for Experiment 2.

Link: https://osf.io/jd96p/

Date registered: 2017-06-21 01:08 AM

Note. Maddock was my married name.

\section{Study Information}

Title

Provide the working title of your study. It is helpful if this is the same title that you submit for publication of your final manuscript, but it is not a requirement.

Does motivation reduce emotional distraction via proactive control mechanisms?

\section{Authors}

The author who submits the preregistration is the recipient of the award money and must also be an author of the published manuscript. Additional authors may be added or removed at any time.

Amy Maddock, Christel Devue, David Carmel, David Harper, Gina M. Grimshaw

\section{Research Questions}

Please list each research question included in this study.

Motivational manipulations cause a reduction in behavioural distraction from positive and negative emotional stimuli (Maddock, Harper, Carmel, and Grimshaw, submitted). It is thought that motivation enhances control in emotionally-neutral contexts via a shift to an anticipatory, proactive control strategy (see Botvinick and Braver, 2015 for a review). However, whether reward elicits a shift towards proactive control to control more potent emotional distractors is unknown. This experiment uses pupillometry as an index of the timing of cognitive control and emotional processing to elucidate the mechanisms underlying the effect of motivation on emotional distraction.

Q. 1 Does motivation enhance control of distractions via a shift towards proactive control that prevents distraction before it occurs?

Q. 2 If so, is this proactive shift implemented on a trial-by-trial basis, and / or is it sustained throughout the task?

Q. 3 Does motivation enhance control of positive, negative and neutral distractions via the same mechanisms? 
Motivation and Emotional Distraction

\section{Hypotheses}

For each of the research questions listed in the previous section, provide one or multiple specific and testable hypotheses. Please state if the hypotheses are directional or nondirectional. If directional, state the direction. A predicted effect is also appropriate here.

Q.1. Does motivation enhance control of distractions via a shift towards proactive control that prevents distraction before it occurs?

Q.2. If so, is this proactive shift implemented on a trial-by-trial basis, or is it sustained throughout the task?

Hypothesis: Motivation increases cognitive control to reduce behavioural distraction. Prediction: A main effect of reward on RT distraction indices, with the reward group being less distracted than controls.

Hypothesis: Motivation reduces distraction via a shift towards a proactive control strategy which is implemented in anticipation of distraction on a trial-by-trial basis. Prediction: A main effect of reward on pre-target phasic pupil dilation (indexing proactive control), with the reward group showing greater proactive control compared to controls.

Hypothesis: Motivation reduces distraction via a shift towards proactive control which is sustained throughout the task.

Prediction: The reward group will show greater tonic pupil dilation compared to the control group, reflecting a greater sustained proactive control due to greater motivation (in line with Chiew and Braver, 2013; 2014).

Note: This may appear to be inconsistent with predictions from Adaptive Gain Theory (AGT; Aston-Jones and Cohen, 2005; see Gilzenrat et al., 2010). AGT posits that tonic pupil dilation (reflecting activity of the locus-coeruleus norepinephrine, LC-NE, system) follows an inverted U-shaped function with task engagement. According to AGT, when tonic LC-NE (and therefore tonic pupil) activity is very high, animals are distractible; when the tonic rate is very low, animals are disengaged and sleepy. Moderate tonic LC-NE activity is a kind of Goldilocks' zone for optimal task focus, with effective filtering of irrelevant stimuli. Presumably our reward group would be in the moderate tonic zone; but we do not know where on the continuum our control group would lie (i.e., are they distractible, or sleepy?). Therefore, it is impossible to make directional hypotheses based on the theory. Moreover, predictions of AGT do not always align with findings from measures of tonic pupil dilation in human participants (e.g. Chiew and Braver, 2013; 2014), and the relationship between tonic pupil dilation and task engagement in humans is likely to be more complex (especially when reward is involved), involving neurotransmitter systems other than LC-NE. If actual findings show the opposite pattern to that of Chiew and Braver, we will consider that an exploratory finding that could be interpreted in terms of AGT.

Hypothesis: A motivation-driven shift towards proactive control prevents distractor processing before it occurs.

Prediction: A main effect of reward on (the difference between intact and scrambled image) post-target phasic pupil dilation, reflecting less processing of intact distractors. 


\section{Motivation and Emotional Distraction}

Q.3. Does motivation enhance control of positive, negative and neutral distractions via the same mechanisms?

Hypothesis: Motivation increases cognitive control of emotional distraction to a greater degree than neutral distraction.

Prediction: Reward will show a quadratic interaction with valence, reducing positive and negative behavioural (RT) distraction to a greater degree than neutral behavioural (RT) distraction.

Hypothesis: Motivation elicits greater trial-by-trial proactive effort to suppress emotional distractors compared to neutral ones.

Prediction: A quadratic reward x valence interaction, reflecting greater relative pretarget phasic pupil dilation (proactive control) for emotional (both positive and negative) compared to neutral blocks in the reward group compared to controls. Alternatively, if it takes greater proactive control to suppress positive than negative distractors (or vice versa), we would find a non-quadratic reward $\mathrm{x}$ valence interaction. There is no predicted direction for a difference between positive and negative proactive control.

Hypothesis: Motivation elicits greater sustained proactive effort to suppress emotional distractors compared to neutral ones.

Prediction: This would be indicated by a quadratic reward $\mathrm{x}$ valence interaction on tonic pupil dilation, reflecting greater relative tonic pupil dilation (sustained proactive control) for positive and negative compared to neutral blocks in the reward group compared to controls. Alternatively, if there is greater sustained proactive effort to suppress positive than negative distractors (or vice versa), we would find a nonquadratic reward $\mathrm{x}$ valence interaction. There is no predicted direction for a difference between sustained proactive control to control positive versus negative distractions. Hypothesis: A motivation-driven shift towards proactive control has a greater effect on control of emotional compared to neutral distraction.

Prediction: A quadratic interaction between reward and valence, reflecting a greater reduction in (the difference between intact and scrambled image) post-target phasic pupil dilation in response to positive and negative compared to neutral distractors. This would indicate that reward has a greater effect on attenuating emotional compared to neutral distraction. Alternatively, if there is greater reward-driven attenuation of positive than negative distraction (or vice versa), we would find a non-quadratic reward $\mathrm{x}$ valence interaction on (intact - scrambled) post-target phasic pupil dilation. There is no predicted direction for a difference between positive and negative post-target pupil dilation. See section 'Statistical models / primary analysis and predictions' and 'Followup analyses' for additional hypotheses and predictions that don't address the key research questions. 
Motivation and Emotional Distraction

\section{Sampling Plan}

\section{Existing Data}

Preregistration is designed to make clear the distinction between confirmatory tests, specified prior to seeing the data, and exploratory analyses conducted after observing the data. Therefore, creating a research plan in which existing data will be used presents unique challenges. Please select the description that best describes your situation. Please do not hesitate to contact us if you have questions about how to answer this question (prereg@cos.io).

Registration prior to creation of data

\section{Explanation of existing data}

If you indicate that you will be using some data that already exist in this study, please describe the steps you have taken to assure that you are unaware of any patterns or summary statistics in the data. This may include an explanation of how access to the data has been limited, who has observed the data, or how you have avoided observing any analysis of the specific data you will use in your study. The purpose of this question is to assure that the line between confirmatory and exploratory analysis is clear.

NA.

\section{Data collection procedures}

Please describe the process by which you will collect your data. If you are using human subjects, this should include the population from which you obtain subjects, recruitment efforts, payment for participation, how subjects will be selected for eligibility from the initial pool (e.g. inclusion and exclusion rules), and your study timeline. For studies that don't include human subjects, include information about how you will collect samples, duration of data gathering efforts, source or location of samples, or batch numbers you will use.

Participants will be recruited from the community via fliers and emails, with an aim of having even numbers of men and women in each group (however, numbers of men and women may differ depending on availability of eligible participants). Participants will be between 18-39 years old, with normal vision, able to speak fluent English, able to use a mouse and computer keyboard, willing to look at gory images and erotica, and will not be currently treated for depression or anxiety. Participants will not have participated in a similar study (using an emotional distraction task) in our lab in the past 3 months, and will not have participated in a similar study in our lab (using an emotional distraction task) with a reward manipulation in the past year. Participants will be paid $\$ 20$ in supermarket vouchers for their time. Participants will be randomly allocated to the control or reward groups. Data collection is expected to take several months. The study is approved by the School of Psychology Human Ethics Committee, by delegated authority of the Victoria University of Wellington Human Ethics Committee. no file selected 
Motivation and Emotional Distraction

\section{Sample size}

Describe the sample size of your study. How many units will be analyzed in the study? This could be the number of people, birds, classrooms, plots, interactions, or countries included. If the units are not individuals, then describe the size requirements for each unit. If you are using a clustered or multilevel design, how many units are you collecting at each level of the analysis?

$\mathrm{n}=54$ per group (108 total).

\section{Sample size rationale}

This could include a power analysis or an arbitrary constraint such as time, money, or personnel.

To determine the sample size needed to find a significant effect of reward on the pupil's arousal response to the emotional images, it was assumed that the effect of reward on post-target pupil dilation would be a similar size to the effect of reward on behavioural RT emotional distraction [((mean RT intact - mean RT scrambled) / mean RT scrambled) x 100]. A sample size analysis was conducted in G Power (Faul, Erdfelder, Lang, and Buchner, 2007) comparing the mean RT emotional distraction indices for the reward group and control group from Maddock et al. (submitted). With an effect size of $d=.519,47$ participants per group are needed to find a significant effect of reward on emotional distraction $80 \%$ of the time (alpha $=.05$ ). A second sample size analysis was conducted as an estimate of the size of the effect of a topdown cognitive control manipulation on the pupil's response to emotional images. Cohen, Moyal and Henik (2015) found that flanker incongruency reduced the pupil's response to negative images (relative to neutral images). A sample size analysis was conducted comparing the mean congruent versus incongruent negative-neutral difference in pupil dilation. With an effect size of $d=.503,50$ participants per group are needed to find a significant effect of congruency on emotional processing $80 \%$ of the time. Rounding up on these two sample size analyses to account for counterbalancing, a sample size of 54 per group was determined. A sensitivity analysis (also in G Power, with alpha $=.05$ ) showed that 54 participants per group is sensitive enough to detect an effect of $d=.482$ or larger $80 \%$ of the time.

\section{Stopping rule}

If your data collection procedures do not give you full control over your exact sample size, specify how you will decide when to terminate your data collection.

If a participant is removed (see exclusion criteria) then they will be replaced. Data will be collected until there are 54 participants in each group. 
Motivation and Emotional Distraction

\section{Variables}

\section{Manipulated variables}

Describe all variables you plan to manipulate and the levels or treatment arms of each variable. For observational studies and meta-analyses, simply state that this is not applicable.

A full description of the task, manipulations and procedure is provided in the attached file 'The Task.doc' Reward: 2 levels (reward and control); between-subjects. Image Type: 2 levels (intact and scrambled); within-subjects. Image Valence: 3 levels (negative, neutral, positive); within-subjects. The images are the distractors. On some trials they are intact, on some they are scrambled. The scrambled trials are a control condition that have the same lower level visual properties as the intact images, but lack meaningful content). On some blocks the images are negative, on some they are positive, and on some they are neutral. On each valence block the scrambled images are versions of the intact images on that block. The reward group receives performance-contingent points; they can achieve levels, which they are told result in extra voucher earnings (all participants actually receive the maximum voucher total). The control group does not receive points, achieve levels, or have extra voucher incentive.

The task_20.06.17.docx

Trial_Diagrams.20.06.17.jpg

\section{Measured variables}

Describe each variable that you will measure. This will include outcome measures, as well as any predictors or covariates that you will measure. You do not need to include any variables that you plan on collecting if they are not going to be included in the confirmatory analyses of this study.

Size of the left pupil (in arbritrary units) will be recorded using an Eyelink 1000-plus desktop mounted eye-tracker using a $1000 \mathrm{~Hz}$ sampling rate. Response times (RTs) and accuracy (proportion correct) will be collected using E Prime 2.0 (Psychology Software Tools, Pittsburgh, PA). Pupil size and RTs will be transformed into indices described in the section 'Indices' below.

no file selected

\section{Indices}

If any measurements are going to be combined into an index (or even a mean), what measures will you use and how will they be combined? Include either a formula or a precise description of your method. If your are using a more complicated statistical method to combine measures (e.g. a factor analysis), you can note that here but describe the exact method in the analysis plan section. 
Motivation and Emotional Distraction

All the following indices are calculated for each individual participant, then are averaged separately for negative, neutral and positive blocks, separately for reward and control groups. All RT and pupil measures use correct trials only (except for tonic pupil dilation, which does not refer to a particular trial, but baseline pre-trial pupil size averaged over entire blocks). See trial timeline (Figure 1 attached) for a visual breakdown of the timing of measurement of pupil indices and their baselines.

RT distraction indices:

o Correct RTs greater than or equal to $200 \mathrm{~ms}$ (those less than $200 \mathrm{~ms}$ are thought to be anticipatory responses) are included in the means.

o [((mean RT intact - mean RT scrambled) / mean RT scrambled) x 100].

o Reflects percentage slowing due to intact distractors.

o Note: a simple difference score between intact and scrambled RTs is not used because of the predicted overall speeding of RTs in the reward group.

Pre-target pupil proactive control:

o Mean pre-target phasic pupil dilation across the $2000 \mathrm{~ms}$ time-window during fixation, immediately preceding the target display. The baseline (mean pupil size in the $200 \mathrm{~ms}$ period immediately before fixation-onset) is subtracted from this phasic response.

o This indexes effortful anticipation of the distractors, reflecting trial-by-trial proactive control.

Early post-target pupil dilation:

o Mean post-target phasic pupil dilation (1800 ms time-window $500-2300 \mathrm{~ms}$ posttarget-onset). The baseline (mean pupil size during the $200 \mathrm{~ms}$ period immediately before target-onset) is subtracted from the phasic response.

o This phasic response for intact trials will be subtracted from the phasic response of scrambled trials. This uses the scrambled images as a baseline to control for low level visual properties (like colour). Thus, this measure reflects arousal due to processing of the meaning of intact images.

o The time window starts $500 \mathrm{~ms}$ after target-onset as piloting of passive viewing of the images showed that effects of meaning (intact versus scrambled) and emotion did not begin to emerge until this time point.

Late post-target pupil dilation:

o Mean post-target phasic pupil dilation (1800 ms time-window $2300-4100$ ms posttarget-onset). The baseline (mean pupil size during the $200 \mathrm{~ms}$ period immediately before target-onset) is subtracted from the phasic response. o This phasic response for intact trials will be subtracted from the phasic response of scrambled trials. This uses the scrambled images as a baseline to control for low level visual properties (like colour). Thus, this measure reflects arousal elicited by processing of the meaning of intact images. 
Motivation and Emotional Distraction

Sustained pupil proactive control:

o Mean tonic pupil dilation for the $1000 \mathrm{~ms}$ before trial-onset. A baseline (the mean pupil size during the $200 \mathrm{~ms}$ period immediately before trial-onset, averaged across all 24 trials in the baseline, non-reward, scrambled-image only block) is subtracted from this tonic measure.

o Indexes sustained arousal and engagement throughout the task relative to a baseline block at the start of the experiment in which participants complete the same task, but without reward, or distraction by intact images.

Timeline 20.06.17.jpg

\section{Design Plan}

\section{Study type}

Please check one of the following statements

Experiment - A researcher randomly assigns treatments to study subjects, this includes field or lab experiments. This is also known as an intervention experiment and includes randomized controlled trials.

\section{Blinding}

Blinding describes who is aware of the experimental manipulations within a study. Mark all that apply.

No blinding is involved in this study.

\section{Study design}

Describe your study design. Examples include two-group, factorial, randomized block, and repeated measures. Is it a between (unpaired), within-subject (paired), or mixed design? Describe any counterbalancing required. Typical study designs for observation studies include cohort, cross sectional, and case-control studies.

This experiment is mixed design with reward (reward, control) as the between-subjects variable and image type (intact, scrambled) and valence (negative, neutral, positive) as within-subjects variables.

Valence block order will be counterbalanced across participants in an ABC fashion. The response keys will be counterbalanced across participants (so 1 is ' $\mathrm{K}$ ' for half the participants and 1 is ' $\mathrm{N}$ ' for half the participants and vice versa).

no file selected

\section{Randomization}

If you are doing a randomized study, how will you randomize, and at what level? 


\section{Motivation and Emotional Distraction}

Participants will be allocated randomly to the control or reward group using a coin flip prior to each session (until the total $\mathrm{n}$ is collected for one group, at which time only the other condition will be run).

\section{Analysis Plan}

\section{Statistical models}

What statistical model will you use to test each hypothesis? Please include the type of model (e.g. ANOVA, multiple regression, SEM, etc) and the specification of the model (this includes each variable that will be included as predictors, outcomes, or covariates). Please specify any interactions that will be tested and remember that any test not included here must be noted as an exploratory test in your final article.

We will conduct 4 separate mixed-model ANOVAs with valence (negative, neutral, positive) as the within-subjects factor and reward group (reward, control) as the between-subjects factor on the following four indices. Note that image type (intact, scrambled) is not a factor because it is included in the transformations into the indices (for RT distraction and post-target pupil measures; see 'Indices' section above), or isn't relevant (for the pre-target pupil proactive control, and tonic dilation measures; see 'Indices' section above).

1. ANOVA on RT distraction indices (indexing RT behavioural distraction) with valence as the within-subjects factor and reward as the between-subjects factor. Note that image type (intact, scrambled) is not a factor because it is included in the transformations into the RT distraction indices.

a. Hypothesis: Emotional content is more distracting than neutral content.

i. Prediction: A quadratic main effect of valence on RT distraction, with positive and negative images being more distracting than neutrals.

ii. Follow-up analyses: If there is no difference between positive and negative RT distraction (confirmed by a two-sided $t$ test) then positive and negative distraction will be averaged together and compared to neutral distraction in a one-sided $t$ test. If there is a difference between positive and negative distraction then they will both be separately compared to neutral distraction (in two one-sided t tests).

b. Hypothesis: Motivation increases cognitive control to reduce distraction.

i. Prediction: A main effect of reward on RT distraction, with the reward group being less distracted than controls.

c. Hypothesis: Motivation increases cognitive control of emotional distraction to a greater degree than neutral distraction.

i. Prediction: Reward will show a quadratic interaction with valence, reducing positive and negative RT distraction to a greater degree than neutral.

ii. Follow-up analyses: If there is no difference between positive and negative RT distraction in the reward or control groups (confirmed by two two-sided t tests), then positive and negative distraction will be averaged together into 'emotional distraction', and two independent one-sided t tests will be conducted comparing the control vs 


\section{Motivation and Emotional Distraction}

reward groups on emotional and neutral distraction separately. If there is a difference between positive and negative distraction then three independent one-sided $t$ tests will be conducted comparing the control vs reward groups on positive, negative and neutral distraction separately.

2. ANOVA on pupil proactive control with valence as the within-subjects factor and reward as the between-subjects factor. Note that image type is not a factor in this analysis because the participant does not know whether the image on a given trial will be scrambled or intact during the pre-target (fixation) period.

a. Hypothesis: Preparation is greater for emotional content than neutral content. i. Prediction: A quadratic main effect of valence on pupil proactive control, with positive and negative blocks eliciting greater pupil proactive control than neutral blocks.

ii. Follow-up analyses: If there is no difference between pupil proactive control on positive and negative trials (confirmed by a two-sided $t$ test) then pupil proactive control for positive and negative trials will be averaged together and compared to pupil proactive control on neutral trials in a one-sided $t$ test. If there is a difference between pupil proactive control on positive and negative trials then they will both be separately compared to pupil proactive control on neutral trials (in two one-sided t tests). iii. Alternatively to the quadratic main effect of valence, there may be a non-quadratic effect of valence on pupil proactive control, indicating greater pupil proactive control for positive compared to negative blocks (or vice versa). There is no predicted direction for this difference. In this case, two one-sided t tests (comparing neutral to negative, and neutral to positive) and one two-sided test (comparing positive to negative) will be conducted.

b. Hypothesis: Motivation enhances cognitive control via a shift towards a proactive control strategy which is implemented on a trial-by-trial basis prior to target and distractor onset.

i. Prediction: A main effect of reward on pupil proactive control, with the reward group showing greater pupil proactive control compared to controls.

c. Hypothesis: Motivation elicits greater (trial-by-trial) proactive effort to suppress emotional distractors compared to neutral ones.

i. Prediction: A quadratic reward x valence interaction, reflecting greater pupil proactive control for positive and negative compared to neutral blocks in the reward group compared to controls.

ii. Follow-up analyses: If there is no difference between pupil proactive control on positive and negative trials in the reward, or control groups (confirmed by two-sided $t$ tests), then pupil proactive control on positive and negative trials will be averaged together into 'emotional pupil proactive control', and two independent one-sided t tests will be conducted comparing the control vs reward groups on emotional and neutral 


\section{Motivation and Emotional Distraction}

pupil proactive control separately. If there is a difference between pupil proactive control on positive and negative trials then three independent one-sided $t$ tests will be conducted comparing the control vs reward groups on positive, negative and neutral pupil proactive control separately.

iii. Alternatively to the quadratic interaction, there may be a non-quadratic interaction between reward and valence on pupil proactive control, indicating greater rewardelicited pupil proactive control on positive compared to negative blocks (or vice versa). There is no predicted direction for this difference. In this case, three independent onesided t tests will be conducted comparing the control vs reward groups on positive, negative and neutral pupil proactive control separately.

3. ANOVA on post-target pupil dilation (indexing intact distractor processing relative to scrambled distractor processing) with valence and time as the within-subjects factors and reward as the between-subjects factor. Note that image type (intact, scrambled) is not a factor because it is included in the transformations into the indices. There are no specific predictions regarding time.

a. Hypothesis: Emotional distractions are more arousing than neutral ones.

i. Prediction: A quadratic main effect of valence on post-target pupil dilation, with positive and negative images eliciting greater post-target pupil dilation than neutral ones.

ii. Follow-up analyses: If there is no difference between positive and negative posttarget pupil dilation (confirmed by a two-sided t test) then positive and negative posttarget phasic pupil dilation will be averaged together and compared to neutral posttarget pupil dilation in a one-sided $t$ test. If there is a difference between positive and negative post-target phasic pupil dilation then they will both be separately compared to neutral post-target pupil dilation (in two one-sided t tests).

iii. Alternatively to the quadratic main effect of valence, there may be a non-quadratic main effect of valence on post-target pupil dilation, indicating greater processing of intact positive compared to negative images (or vice versa). There is no predicted direction for this difference. In this case, two one-sided t tests (comparing neutral to negative, and neutral to positive) and one two-sided test (comparing positive to negative) will be conducted.

b. Hypothesis: A motivation-driven shift towards proactive control prevents distraction before it occurs (see DMC framework, Braver et al., 2007).

i. Prediction: A main effect of reward on post-target pupil dilation, reflecting less processing of the intact distractors in the reward group relative to the control group.

c. Hypothesis: A motivation-driven shift towards proactive control has a greater effect on emotional compared to neutral distraction. 


\section{Motivation and Emotional Distraction}

i. Prediction: An interaction between reward and valence, reflecting a greater reduction in post-target pupil dilation in response to positive and negative compared to neutral distractors.

ii. Follow-up analyses: If there is no difference between post-target pupil dilation on positive and negative trials in the reward or control groups (confirmed by two-sided $t$ tests), then post-target pupil dilation on positive and negative trials will be averaged together into 'emotional post-target pupil dilation', and two independent one-sided t tests will be conducted comparing the control vs reward groups on post-target pupil dilation on emotional and neutral trials separately. If there is a difference between posttarget pupil dilation on positive and negative trials then three independent one-sided $t$ tests will be conducted comparing the control vs reward groups on positive, negative and neutral post-target pupil dilation separately.

iii. Alternatively to the quadratic interaction, there may be a non-quadratic interaction between reward and valence on post-target phasic pupil dilation, indicating greater reward-elicited reductions in processing of positive compared to negative intact distractors (or vice versa). There is no predicted direction for this difference. In this case, three independent one-sided t tests will be conducted comparing the control vs reward groups on positive, negative and neutral pre-target phasic pupil dilation separately.

4. ANOVA on tonic pupil dilation (indexing sustained proactive control) with valence as the within-subjects factor and reward as the between-subjects factor. Note that image type is not a factor in this analysis because tonic dilation does not refer to a particular trial, but an entire valence block.

a. Hypothesis: Motivation enhances cognitive control via a shift towards a proactive control strategy which is sustained throughout the task.

i. Prediction: A main effect of reward would indicate that reward group shows greater tonic pupil dilation relative to the control group, reflecting motivation-induced sustained proactive effort.

b. Hypothesis: Motivation elicits greater sustained proactive effort to suppress emotional distractors compared to neutral ones.

i. Prediction: A quadratic reward $\mathrm{x}$ valence interaction on tonic pupil dilation, reflecting greater relative tonic pupil dilation (sustained arousal) for positive and negative compared to neutral blocks in the reward group compared to controls. ii. Follow-ups: If there is no difference between the tonic pupil dilation for positive and negative blocks in the reward, or control groups (confirmed by two two-sided t tests), then positive and negative tonic pupil dilation will be averaged together into 'emotional tonic pupil dilation', and two independent one-sided t tests will be conducted comparing the control vs reward groups on emotional and neutral tonic pupil dilation separately. If there is a difference between positive and negative tonic pupil dilation 
Motivation and Emotional Distraction

then three independent one-sided t tests will be conducted comparing the control vs reward groups on positive, negative and neutral tonic pupil dilation separately.

iii. Alternatively to the quadratic interaction, there may be a non-quadratic interaction between reward and valence on tonic pupil dilation, indicating greater reward-elicited sustained proactive control in positive compared to negative blocks (or vice versa). There is no predicted direction for this difference. In this case, three independent onesided t tests will be conducted comparing the control vs reward groups on positive, negative and neutral tonic pupil dilation separately.

c. Hypothesis: Higher sustained arousal in emotional compared to neutral blocks.

i. Prediction: A quadratic valence main effect on tonic dilation, indicating greater tonic pupil dilation in positive and negative compared to neutral blocks. This would reflect greater arousal due to the emotional content, and could reflect greater sustained proactive effort on emotional blocks regardless of reward.

ii. Follow-ups: If there is no difference between positive and negative tonic pupil dilation (confirmed by a two-sided $t$ test) then positive and negative tonic pupil dilation will be averaged together and compared to neutral tonic pupil dilation in a one-sided $t$ test. If there is a difference between positive and negative tonic pupil dilation then they will both be separately compared to neutral tonic pupil dilation (in two one-sided $t$ tests).

iii. Alternatively to the quadratic main effect of valence, there may be a non-quadratic main effect of valence on tonic pupil dilation, indicating greater arousal on positive compared to negative blocks (or vice versa). There is no predicted direction for this difference. In this case, two one-sided t tests (comparing neutral to negative, and neutral to positive) and one two-sided test (comparing positive to negative) will be conducted. For completeness, and to explore overall performance and a possible speedaccuracy trade-off, mixed-model ANOVAs will also be performed on untransformed correct mean RTs and mean accuracy (proportion correct) with valence (negative, neutral, positive) and image type (intact or scrambled) as within-subjects factors and reward (reward, control) as the between-subjects factor.

no file selected

\section{Transformations}

If you plan on transforming, centering, recoding the data, or will require a coding scheme for categorical variables, please describe that process.

Behavioural measures:

Trials with no response are counted as errors.

Only correct responses included in RT means.

Only RTs equal to or greater than $200 \mathrm{~ms}$ included in RT analyses and indices as those faster than $200 \mathrm{~ms}$ are thought to be anticipatory.

Pupil data pre-processing will be performed in MATLAB:

Only correct responses (with RTs equal to or greater than $200 \mathrm{~ms}$ ) will be included in pupil analyses. 
Motivation and Emotional Distraction

Blinks will be linearly interpolated.

Partial blinks will be identified, and the velocity flattened.

Baselining: A $200 \mathrm{~ms}$ mean baseline will be subtracted from phasic responses.

Subtraction was chosen as it is more robust to baseline artifacts, and increases

statistical power, relative to divisive baseline corrections; Mathot, Fabius, Van

Heusden, and Van der Stigchel (in preparation).

For both post-target phasic time-windows, the baseline will be the mean pupil size 200 ms immediately preceding target-onset.

For the pre-target phasic response (during fixation), the baseline will be the mean pupil size $200 \mathrm{~ms}$ pre-fixation-onset.

A grand mean of pupil size in the $200 \mathrm{~ms}$ time window immediately before fixationonset from the baseline (non-reward, scrambled-image only) block will be subtracted from the tonic pupil measure.

\section{Follow-up analyses}

If not specified previously, will you be conducting any confirmatory analyses to follow up on effects in your statistical model, such as subgroup analyses, pairwise or complex contrasts, or follow-up tests from interactions? Remember that any analyses not specified in this research plan must be noted as exploratory.

See Statistical Models section above.

\section{Inference criteria}

What criteria will you use to make inferences? Please describe the information you'll use (e.g. specify the p-values, Bayes factors, specific model fit indices), as well as cut-off criterion, where appropriate. Will you be using one or two tailed tests for each of your analyses? If you are comparing multiple conditions or testing multiple hypotheses, will you account for this?

$P$ values less than .05 will be accepted as statistically significant and relevant follow-up analyses will be conducted on these effects.

Trends (less than .10) will be followed up if they are predicted.

One or two tailed tests are specified in the predictions (Statistical Models, above).

Planned comparisons will not be corrected for multiple comparisons (see Armstrong, 2014). Exploratory analyses (e.g. correlations between pupil and behavioural measures) for hypothesis generating will not be corrected for multiple comparisons, but will be interpreted with caution. If they are reported in the manuscript, the number of tests conducted will also be reported.

\section{Data exclusion}

How will you determine which data points or samples (if any) to exclude from your analyses? How will outliers be handled?

If a trial is missing more than $50 \%$ of its pupil size samples, the trial will be excluded from all pupil analyses. 
Motivation and Emotional Distraction

If a $200 \mathrm{~ms}$ baseline is missing greater than $50 \%$ of the pupil size samples, that trial will be excluded from the pupil analyses that use that particular baseline.

If a participant has less than $75 \%$ accuracy overall, or less than $60 \%$ accuracy on a given block, they will be removed from analyses (and replaced).

If a participant is missing more than $20 \%$ of their pupil size samples overall, they will be removed from analyses (and replaced).

Participants must have 10 or more valid (meeting the criteria above) trials per condition or the participant will be removed from analyses (and replaced).

\section{Missing data}

How will you deal with incomplete or missing data?

Missing pupil samples and missing RTs (due to errors or non-responses) will not be imputed (except for linear interpolation of blinks). Means will include available samples and trials. Participants and trials with missing samples over a certain threshold will be removed (see Data exclusions, above).

\section{Exploratory analysis}

If you plan to explore your data set to look for unexpected differences or relationships, you may describe those tests here. An exploratory test is any test where a prediction is not made up front, or there are multiple possible tests that you are going to use. A statistically significant finding in an exploratory test is a great way to form a new confirmatory hypothesis, which could be registered at a later time.

Possible relationships between scores on the BAS-Drive subscale (which measures the extent to which an individual is willing to work for rewards) of the Behavioural Inhibition and Activation Scale (BIS/BAS; Carver and White, 1994) and reward-related differences in distraction or pupil dilation will be explored. It is predicted that scores on the BAS-Drive (reflecting individual differences in reward sensitivity) may be correlated with behavioural distraction and / or pupil dilation measures in the reward group but not the control group. This would suggest that individual differences in reward sensitivity may influence the extent to which people shift to a proactive strategy to ignore distractors. We also acknowledge that our study was not designed to probe individual differences and sample size may be inadequate to detect such correlations. Correlations between pupil indices and behavioural indices will be explored in order to generate future hypotheses. 
Motivation and Emotional Distraction

Appendix E. These figures contain the output of the functional $t$-tests.

Figure S.1. A full explanation of functional data analysis (FDA) is included in Chapter 3. This figure compares the timecourse of pupil dilation between the reward and control groups for the $2000 \mathrm{~ms}$ pre-stimulus period (indexing dynamic proactive control). The functional $t$ test reveals that the control and reward groups prepared to ignore the distractors over different timecourses. Specifically, the control group exerted effort earlier, and less intensely, with a small boost of control close to stimulus-onset. In contrast, the reward group prepared later, but more intensely, with a large dynamic boost of control close in time to stimulusonset.

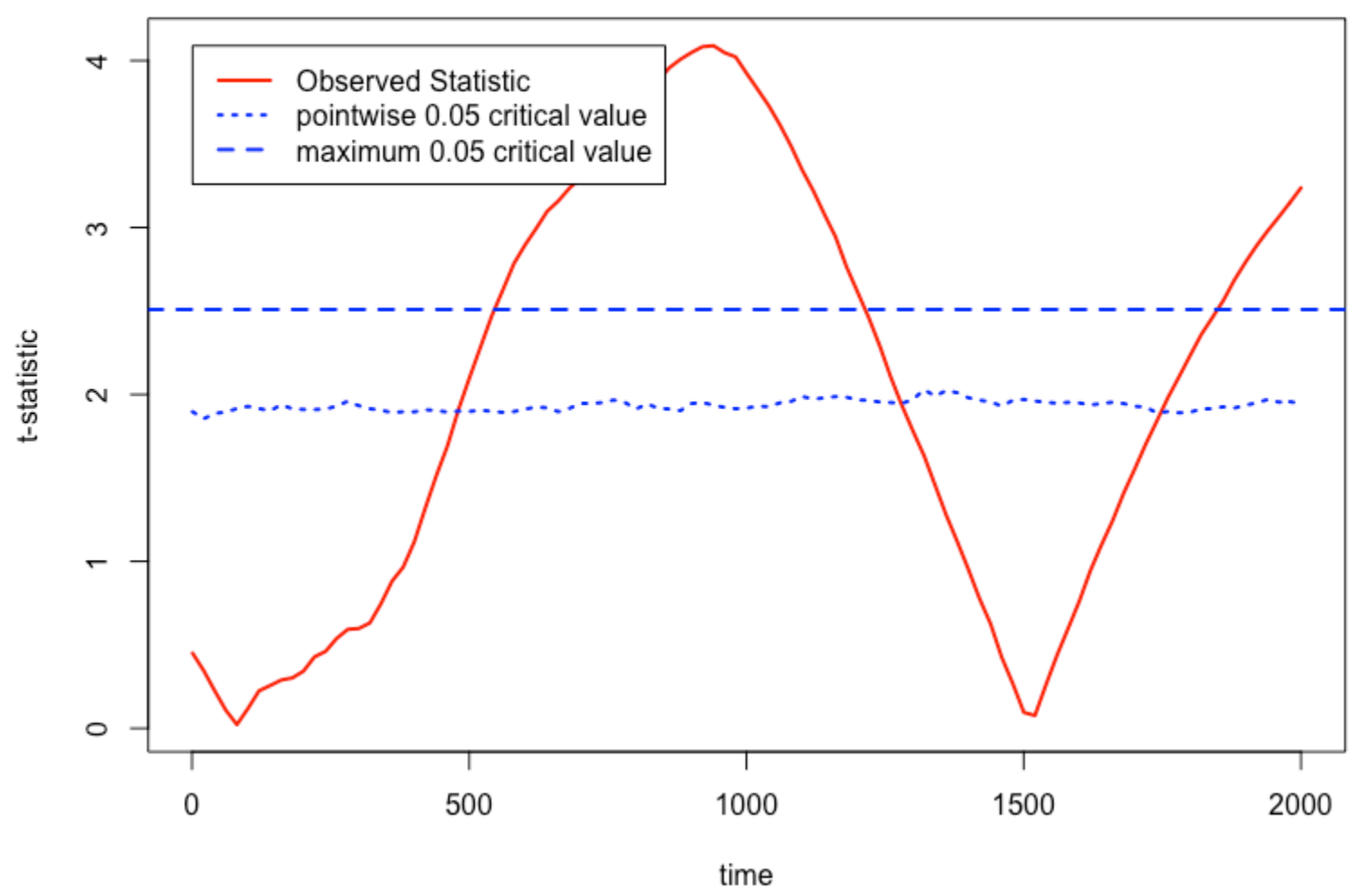

Figure S.1. Functional data analysis (FDA) output across the entire $2000 \mathrm{~ms}$ pre-stimulus window (from fixation onset to stimulus-onset). When the red line (the observed $t$ statistic) is greater than the dotted line (the pointwise critical value) the group difference is significant at that time point. 
Motivation and Emotional Distraction

Figure S.2. This figure compares the timecourse of pupil dilation between the reward and control groups for the post-stimulus period (indexing distractor processing). Findings indicate that reward reduced distractor processing (extending from around 1000 to $2400 \mathrm{~ms}$ poststimulus).

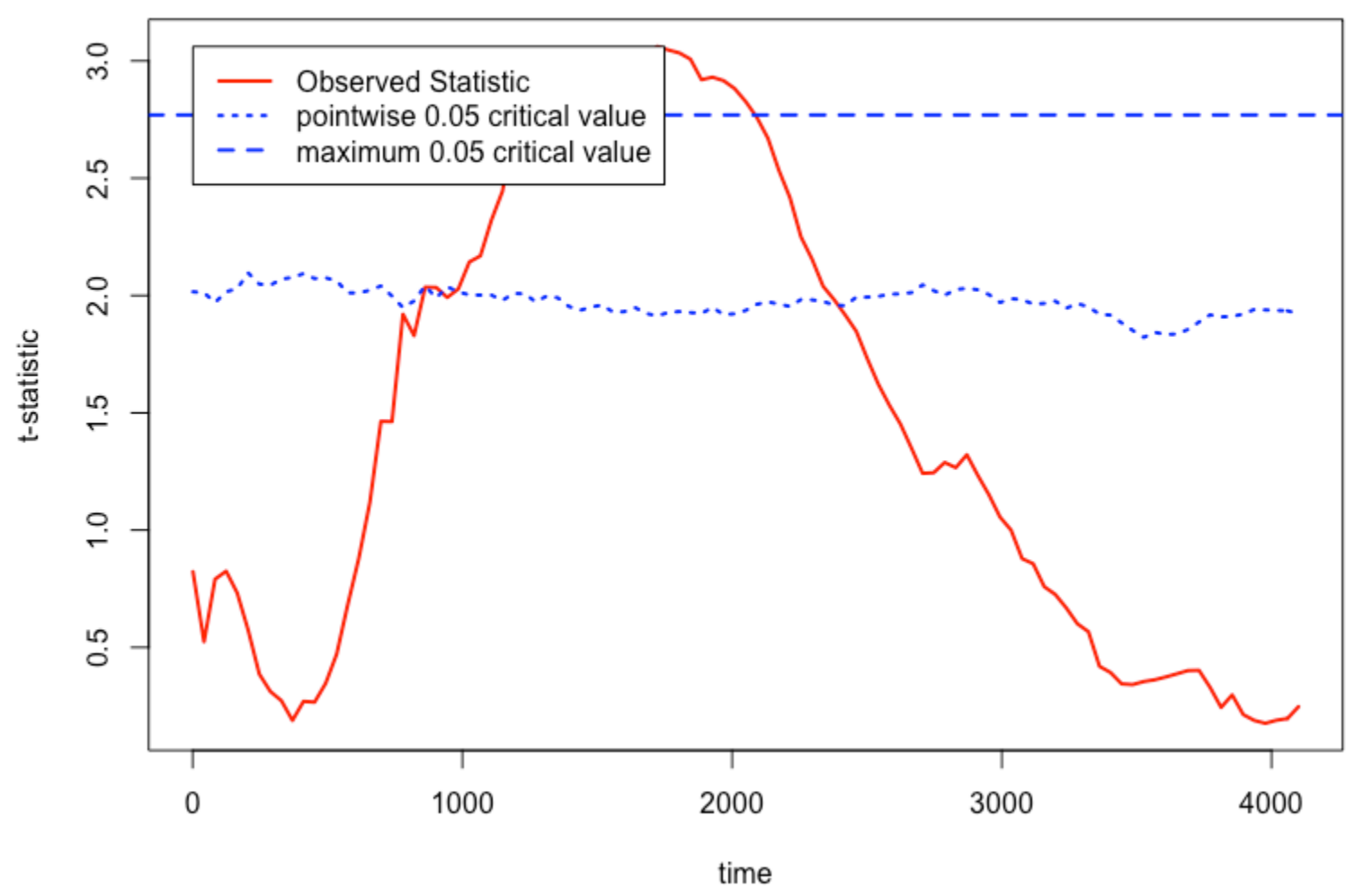

Figure S.2. FDA output for the [intact - scrambled] difference, across the entire $4100 \mathrm{~ms}$ post-stimulus-onset window (from stimulus-onset until the end of the ITI). When the red line (the observed tstatistic) is greater than the dotted line (the pointwise critical value) the group difference is significant at that time point. 
Motivation and Emotional Distraction

Figure S.3. This figure compares the timecourse of pupil dilation between the reward and control groups for the post-stimulus period for intact distractor trials only. Findings indicate that reward increased dilation in response to intact distractors (extending from 0 to around $1200 \mathrm{~ms}$ post-stimulus).

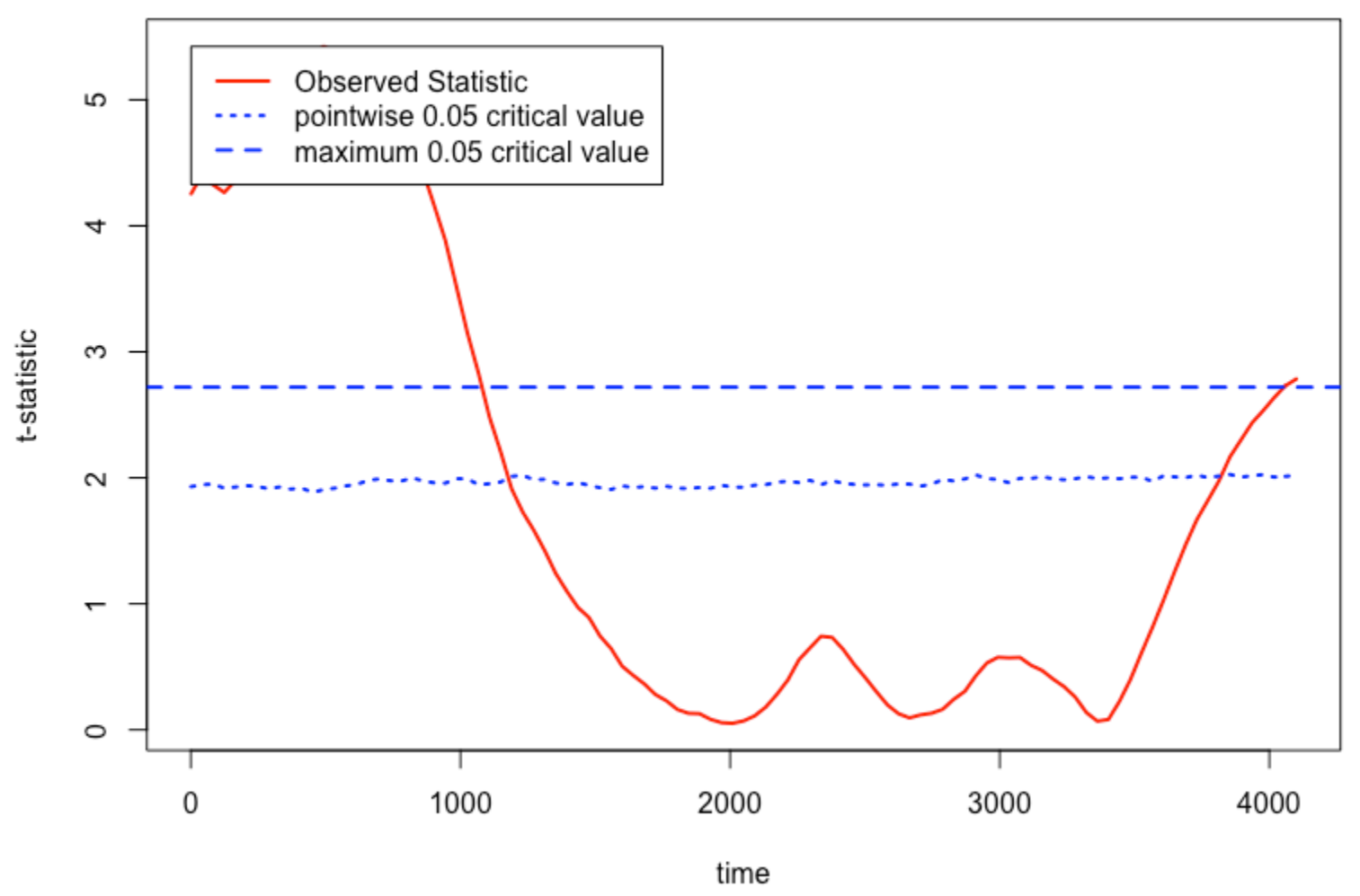

Figure S.3. FDA output for the intact distractor condition, across the entire $4100 \mathrm{~ms}$ poststimulus-onset window (from stimulus-onset until the end of the ITI). When the red line (the observed $t$ statistic) is greater than the dotted line (the pointwise critical value) the group difference is significant at that time point. 
Motivation and Emotional Distraction

Figure S.4. This figure compares the timecourse of pupil dilation between the reward and control groups for the post-stimulus period for scrambled distractor trials only. Findings indicate that reward increased dilation in response to scrambled distractors (extending from 0 to around $2550 \mathrm{~ms}$ post-stimulus).

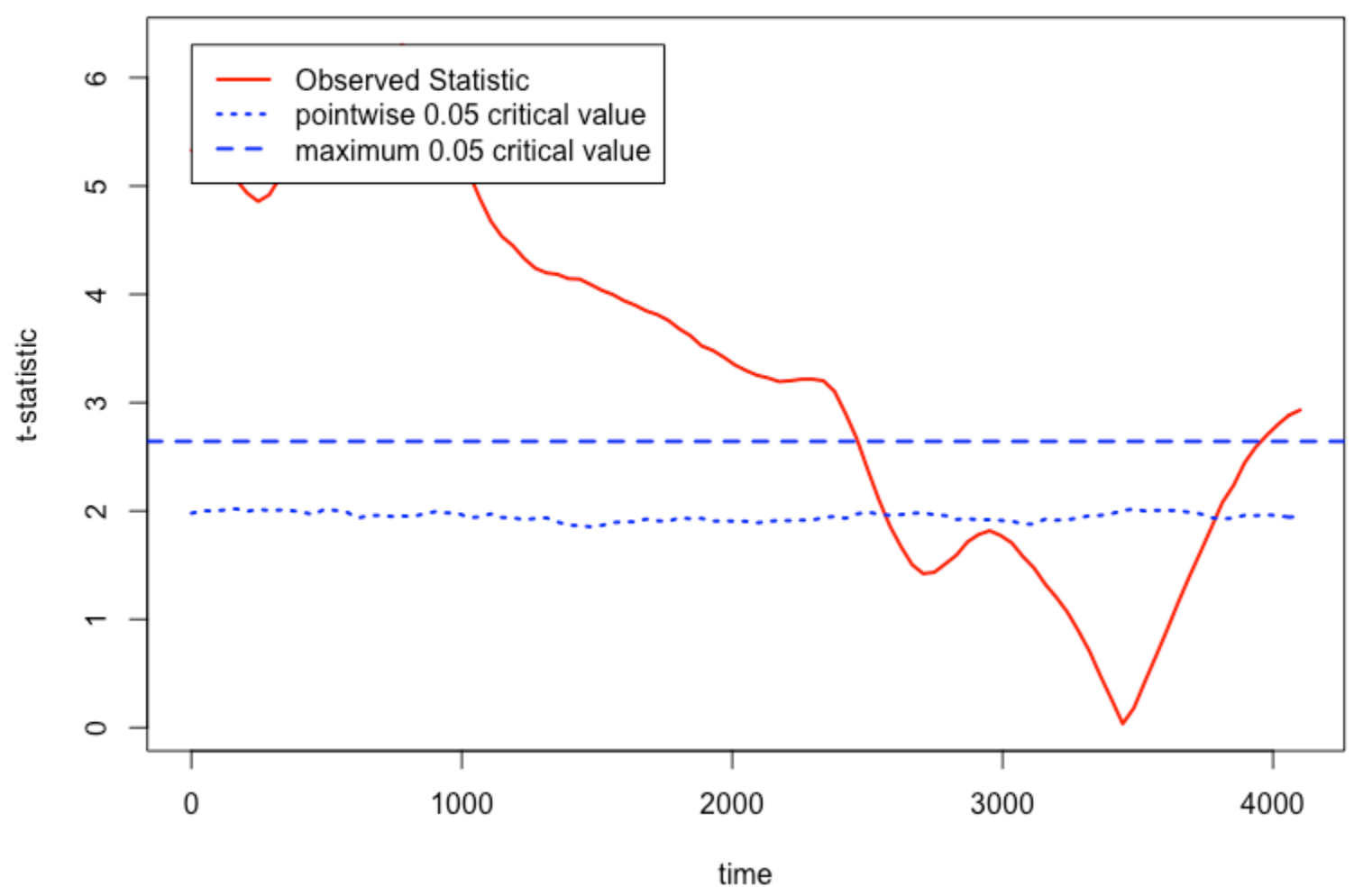

Figure S.4. FDA output for the scrambled distractor condition, across the entire $4100 \mathrm{~ms}$ post-stimulus-onset window (from stimulus-onset until the end of the ITI). When the red line (the observed t statistic) is greater than the dotted line (the pointwise critical value) the group difference is significant at that time point. 
Motivation and Emotional Distraction

Appendix F. AsPredicted.org Preregistration for Experiment 4.

(\#9627) Created: 04/04/2018 08:39 PM (PT)

Author(s)

Amy Walsh (Victoria University of Wellington) - amy.walsh@vuw.ac.nz

Gina Grimshaw (Victoria University of Wellington) - gina.grimshaw@vuw.ac.nz

Petra Bolitho (Victoria University of Wellington) - petrabolitho@gmail.com

\section{1) Have any data been collected for this study already?}

No, no data have been collected for this study yet.

2) What's the main question being asked or hypothesis being tested in this study?

Does symbolic (non-monetary) reward reduce emotional distraction?

3) Describe the key dependent variable(s) specifying how they will be measured.

Mean correct RTs $(<200 \mathrm{~ms})$ for each condition.

Mean proportion correct within a condition.

4) How many and which conditions will participants be assigned to?

- Reward (reward, control); between subjects (random assignment).

- Distractor presence (25\% present, $75 \%$ absent); within-subjects.

- Distractor emotion (emotional, neutral); blocked, within-subjects.

- Valence superblock (positive, negative). Positive (and neutral) images are presented in one superblock, negative (and neutral) images in the other.

Assuming that RTs and accuracy do not significantly differ across positive and negative superblocks (in a two-tailed $t$ test), neutral responses will be collapsed, combining distractor emotion and superblock valence factors into distractor valence (negative, neutral, positive). If they do differ, distractor emotion and superblock valence will be treated separately.

\section{5) Specify exactly which analyses you will conduct to examine the main question/hypothesis.}

A 2 × 2 × 3 ANOVA will be conducted on correct RTs with reward group (reward, control) as the between-subjects factor, and distractor presence (absent, present) and distractor valence (negative, neutral, positive) as within-subjects factors. Importantly, if reward enhances control, we predict a reward $\mathrm{x}$ distractor presence interaction.

We predict a distractor presence $\mathrm{x}$ valence interaction (greater distraction on positive and negative than neutral blocks). We predict a main effect of reward (faster responses under reward). If reward enhances control of emotional more than neutral distraction we predict a reward $\mathrm{x}$ distractor presence $\mathrm{x}$ valence interaction. Interactions with distractor presence will be followed up with two-tailed t-tests comparing distraction (distractor present - distractor absent RTs) between conditions.

6) Describe exactly how outliers will be defined and handled, and your precise rule(s) 
Motivation and Emotional Distraction

for excluding observations.

Participants with $<75 \%$ accuracy overall, or $<60 \%$ accuracy on a single block will be eliminated and replaced. Participants with distraction 4 SDs $<$ or $>$ the mean for a given condition will be eliminated and replaced.

\section{7) How many observations will be collected or what will determine sample size?}

No need to justify decision, but be precise about exactly how the number will be determined. A sensitivity analysis ( $\mathrm{G}^{*}$ Power) at $80 \%$ power $(\mathrm{a}=.05$, one-tailed) found that 48 participants per group can detect an effect of reward on distraction of $d=.511$. We will run participants until we have 48 per group.

\section{8) Anything else you would like to pre-register?}

(e.g., secondary analyses, variables collected for exploratory purposes, unusual analyses planned?)

We will conduct an equivalence test using the smallest effect size we can detect at $80 \%$ power as the lower and upper bounds $(\mathrm{a}=.05$, two-tailed), to examine whether the group difference is similar for positive and negative blocks.

A $2 \times 2 \times 3$ ANOVA (as above), will be conducted with proportion correct as the DV. We predict a main effect of reward; more errors in the reward compared to the control group. We predict a main effect of distractor presence, and a distractor presence $\mathrm{x}$ distractor valence interaction (emotional distraction).

To check that a speed-accuracy trade-off does not account for the effect of reward on emotional distraction, we will conduct an ANOVA on inverse-efficiency scores $[\mathrm{RT} /$ proportion correct]. We predict that the distractor presence $\mathrm{x}$ reward interaction will remain significant.

Responses on the Behavioural Activation and Inhibition Scale (BIS/BAS; Carver \& White, 2013) will be collected to explore possible relationships between reward, distraction, and reward sensitivity to generate future hypotheses. 
Motivation and Emotional Distraction

Appendix G. Supplementary analysis of the influence of the shared reward criterion across different distractor valence blocks.

Table S.2. Summary of rewarded trials for each condition for Experiment 1. The first three rows show the mean, minimum, and maximum percentage of rewarded trials across participants in the reward group, for each valence and distractor type condition. The fourth row shows the number of participants who achieved a level of $90 \%$ rewarded trials. The last row shows how much faster participants were than their individual median $R T$ threshold, on average, in milliseconds (ms). Findings show that, although the reward criterion was derived from non-emotional trials, it was still an effective reward contingency on neutral distractor blocks.

\begin{tabular}{|c|c|c|c|c|c|c|c|c|}
\hline & \multicolumn{4}{|c|}{ Negative Super-block } & \multicolumn{4}{|c|}{ Positive Super-block } \\
\hline & \multicolumn{2}{|c|}{ Negative Block } & \multicolumn{2}{|c|}{ Neutral Block } & \multicolumn{2}{|c|}{ Neutral Block } & \multicolumn{2}{|c|}{ Positive Block } \\
\hline & $\begin{array}{c}\text { Scram- } \\
\text { bled }\end{array}$ & Intact & $\begin{array}{c}\text { Scram- } \\
\text { bled }\end{array}$ & Intact & $\begin{array}{c}\text { Scram- } \\
\text { bled }\end{array}$ & Intact & $\begin{array}{c}\text { Scram- } \\
\text { bled }\end{array}$ & Intact \\
\hline $\begin{array}{l}\text { Mean \% } \\
\text { rewarded } \\
\text { trials }\end{array}$ & $75 \%$ & $59 \%$ & $75 \%$ & $67 \%$ & $75 \%$ & $69 \%$ & $74 \%$ & $62 \%$ \\
\hline $\begin{array}{l}\text { Min \% } \\
\text { rewarded } \\
\text { trials }\end{array}$ & $57 \%$ & $21 \%$ & $50 \%$ & $33 \%$ & $51 \%$ & $33 \%$ & $49 \%$ & $25 \%$ \\
\hline $\begin{array}{l}\text { Max \% } \\
\text { rewarded } \\
\text { trials }\end{array}$ & $96 \%$ & $92 \%$ & $97 \%$ & $88 \%$ & $94 \%$ & $100 \%$ & $96 \%$ & $92 \%$ \\
\hline $\begin{array}{l}n \\
\text { rewarded } \\
\text { over } 90 \% \\
\end{array}$ & $n=4$ & $n=1$ & $n=4$ & $n=0$ & $n=3$ & $n=5$ & $n=1$ & $n=1$ \\
\hline $\begin{array}{l}\text { Individual } \\
\text { median } \\
\text { RT minus } \\
\text { mean RT }\end{array}$ & $90 \mathrm{~ms}$ & $37 \mathrm{~ms}$ & $87 \mathrm{~ms}$ & $63 \mathrm{~ms}$ & $89 \mathrm{~ms}$ & $70 \mathrm{~ms}$ & $86 \mathrm{~ms}$ & $45 \mathrm{~ms}$ \\
\hline
\end{tabular}


Motivation and Emotional Distraction

Appendix H. Supplementary analysis of the influence of image repetition in Chapter 2.

We conducted an ANOVA on RTs with distractor type (intact, scrambled) and block $(2,3)$ as within-subjects factors and reward as the between-subjects factor, for Experiment 1. Using responses from blocks 2 and 3 allowed us to examine responses on trials with the first and second presentation of distractor images of the same valence (within a participant; but images of different valences across participants, because of the ABBA counterbalance order), while limiting the overall effect of the reduction in distraction across the whole experiment. Main effects of distractor type and block were qualified by a significant distractor type $\times$ block interaction (reflecting attenuation of distraction on the second ( $M=38 \mathrm{~ms}, S D=46 \mathrm{~ms}$ ) compared to the first presentation $(M=66 \mathrm{~ms}, S D=75 \mathrm{~ms})$ of the distractors, $F(1,70)=$ $10.80, p=.002, \eta_{p}{ }^{2}=0.13$. This suggests that there is an effect of image repetition on distraction, in which distraction is reduced on second viewing of the images. But importantly, there were no interactions between reward $\times$ block $\left(p=.295, \eta_{p}{ }^{2}=.02\right)$, or reward $\times$ block $\times$ distractor type $\left(p=.513, \eta_{p}^{2}=.01\right)$. Therefore repetition effects cannot account for findings regarding the benefit of reward on distraction. 